Tous les enfants à l'école d'ici 2015

Initiative mondiale en faveur des enfants non scolarisés

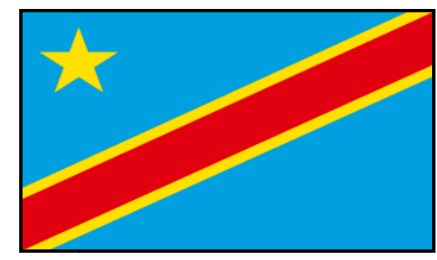

République Démocratique du Congo

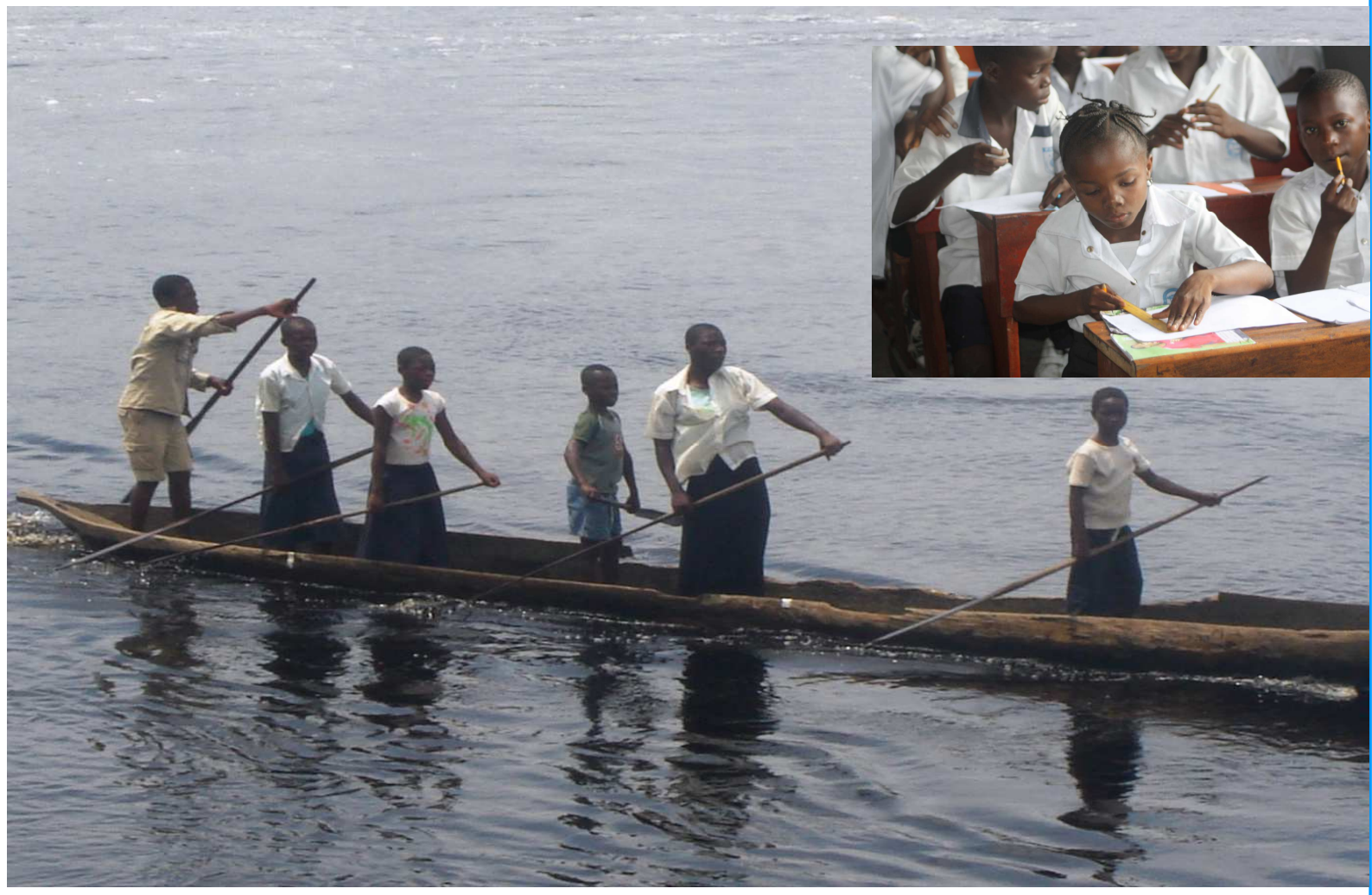

Rapport de l'enquête nationale sur les enfants et adolescents en dehors de l'école

Février 2013
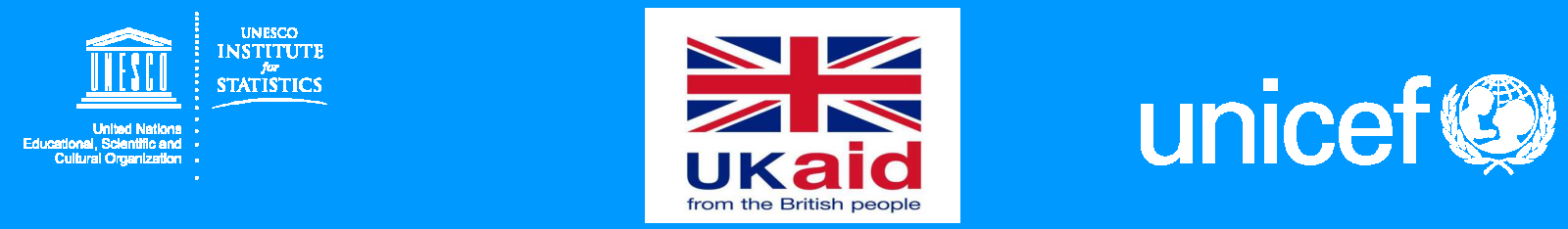
Ce rapport présente les résultats de l'enquête nationale sur la situation des enfants et adolescents en dehors de l'école en République Démocratique du Congo (EADE-RDC) de 2012, organisée par le Ministère de l'Enseignement Primaire, Secondaire et Professionnel et exécutée par l'Institut Supérieur des Sciences de la Population de l'Université de Ouagadougou (ISSP/UO). L'EADE-RDC a été réalisée avec l'appui financier du Département britannique pour le Développement International (DFID) et de l'appui technique du Fonds des Nations Unies pour l'Enfance (UNICEF) et I'Institut de Statistique de I'UNESCO. 


\section{Sigles et abréviations}

\begin{tabular}{|c|c|}
\hline BAD & Banque Africaine de Développement \\
\hline BM & Banque Mondiale \\
\hline CDE & Convention relative aux droits de l'enfant \\
\hline CITE & Classification Internationale Type de l'Education \\
\hline CNE & Comité National de l'Enfant \\
\hline CMF & Conceptual and Methodological Framework (cadre conceptuel et méthodologique) \\
\hline CNEN & Conseil National de l'Enfant \\
\hline CRC & Children Rights Committee (Comité des droits de l'enfant) \\
\hline CSP & Cycle Spécialisation Professionnelle \\
\hline DFID & UK Department For International Development \\
\hline DSCRP & Document des Stratégies de Croissance et de Réduction de la Pauvreté \\
\hline EADE & Enfants et Adolescents en Dehors de l'Ecole \\
\hline EDS & Enquête Démographique et de Santé \\
\hline ENHAPS & Enquête Nationale sur l'Habitat \\
\hline EPSP & Enseignement Primaire, Secondaire et Professionnelle \\
\hline ESU & Enseignement Supérieur et Universitaire \\
\hline FMI & Fonds Monétaire International \\
\hline IDA & International Development Association (Association internationale de développement) \\
\hline INS & Institut National de la Statistique \\
\hline ISSP & Institut Supérieur des Sciences de la Population \\
\hline ISU (UIS) & Institut de Statistique de I'UNESCO (UNESCO Institute for Statistics) \\
\hline MAS & Ministère des Affaires sociales \\
\hline MICS & Multiple Indicators Cluster Survey (Enquête par grappes à indicateurs multiples) \\
\hline MINAS & Ministère des affaires sociales, action humanitaire et solidarité nationale \\
\hline MONUC & Mission d'observation des Nations Unies au Congo \\
\hline MONUSCO & Mission d'observation des Nations Unies pour la Stabilisation du Congo \\
\hline ND & Non déclaré \\
\hline OEV & Orphelins et Enfants Vulnérables \\
\hline OIM-RDC & Organisation Internationale de la Migration en République Démocratique du Congo \\
\hline OIT & Organisation Internationale du Travail \\
\hline ONG & Organisation Non Gouvernementale \\
\hline OSC & Organisations de la société civile \\
\hline PIB & Produit Intérieur Brut \\
\hline PNMLS & Programme National Multisectoriel de Lutte contre le Sida \\
\hline PPTE & Pays Pauvres Très Endettés \\
\hline PRONANUT & Programme National de Nutrition \\
\hline PROVED & Province Educationnelle \\
\hline RDC & République Démocratique du Congo \\
\hline RESEN & Rapport sur l'Etat du Système Educatif National \\
\hline SG & Secrétaire Général \\
\hline UCW & Understanding Children's Work \\
\hline UE & Union Européenne \\
\hline UNDP & United Nations Development Programme \\
\hline UNICEF & Fonds des Nations Unies pour l'Enfance \\
\hline USD & United States Dollar (dollar américain) \\
\hline
\end{tabular}




\section{Avant propos}

L'éducation est un levier essentiel du développement, particulièrement dans le contexte de la République Démocratique du Congo (RDC) où l'Etat s'est engagé, depuis plusieurs années, avec l'accompagnement des communautés et des partenaires au développement, dans un processus de pacification, de relance de l'économie et d'amélioration des conditions de vie des populations.

Procurer à tous les enfants, adolescents et jeunes de la RDC une éducation de qualité répond non seulement au souci de satisfaire un droit humain fondamental, mais aussi d'offrir des opportunités à des millions de citoyens congolais de sortir de la pauvreté et de contribuer ainsi, de manière substantielle, au développement de la Nation.

II s'agit là d'une question prioritaire pour l'Etat congolais, comme en témoigne la Constitution du 18 février 2006 qui, à travers plusieurs articles, consacre le caractère obligatoire et gratuit de l'enseignement primaire dans les établissements publics, affirme le principe de non-discrimination en matière d'éducation et édicte la nécessité d'éradiquer l'analphabétisme. Par ailleurs, le Document de Stratégie de développement du soussecteur de l'enseignement primaire, secondaire et professionnel 2010/2011-2015/2016 adopté en mars 2010, dont l'objectif général est de " construire un système éducatif inclusif et de qualité ", consacre ce principe de la gratuité et du caractère obligatoire de l'enseignement primaire. Principe qui va, la même année 2010, se traduire par le lancement, par son Excellence Monsieur Joseph Kabila, Président de la République, de la politique de gratuité de l'enseignement primaire, d'abord au niveau des trois premières années du primaire, avant son extension progressive sur l'ensemble du cycle primaire.

Certes, des progrès ont été réalisés au cours des dernières années en matière d'éducation en RDC. Cependant, force est de reconnaitre que des défis persistent en termes de production des statistiques scolaires fiables reposant sur des évidences scientifiques pour faciliter la prise de décisions. S'y rajoutent le développement des méthodologies de recherche et la mise en place des mécanismes de collecte et traitement de données efficaces.

L'étude sur les Enfants et adolescents en dehors de l'école, objet du présent rapport, vient combler ce déficit. A l'instar des autres études du même genre, entreprises dans 26 pays dans le cadre de l'Initiative mondiale en faveur des enfants non scolarisés, la présente étude fournit des estimations précises de l'ampleur du phénomène des enfants et adolescents en dehors de l'école, dégage le profil de ces enfants, identifie les obstacles et goulots d'étranglement à leur scolarisation et propose des pistes de solutions pour leur insertion ou réinsertion dans le système éducatif.

Elle constitue une mine d'informations précieuses qui arrive à point nommé et dont, j'en suis convaincu, l'ensemble des acteurs du développement s'inspireront dans leurs efforts pour l'amélioration de l'équité, de l'efficacité et de l'efficience du système éducatif congolais afin que chaque enfant et adolescent congolais accède à une éducation de qualité.

Maker Mwangu Fwamba

Ministre de l'Enseignement primaire, secondaire et professionnel 


\section{Remerciements}

L'étude sur les Enfants et Adolescents en Dehors de l'Ecole en République Démocratique du Congo (EADE$R D C$ ) a été réalisée grâce au soutien financier, technique et logistique de plusieurs institutions et à l'implication de plusieurs personnes.

C'est pourquoi, nous remercions très sincèrement la Coopération britannique - DFID (Felicity Townsend, Janice Dolan, Gabu Kongolo N'singa) pour son appréciable appui financier et technique à l'étude ainsi que I'UNICEF pour le suivi de la mise en œuvre de l'étude, pour son apport technique et sa contribution à l'organisation (Barbara Bentein et Sylvie Fouet pour leur plaidoyer, Cecilia Baldeh, Ibrahima Diallo, Pascaline Mupaya, Yumiko Yokozeki, Dina Craissati et Tahina Razafindramary pour leurs contributions techniques et le pilotage de l'étude).

Nous exprimons également notre reconnaissance à l'Institut de statistique de I'UNESCO (Thierry Lairez, Sheena Bell et Friedrich Huebler) pour son appui technique de l'étude, au Comité technique interministériel (Jovin Mukadi, François Kubindikila, Jean Bosco Kelekele, Joseph Kenseka, Jean Baptiste Mbaya Kashala, Henri Bobunda, Botiho Kumi, Marcel Ndiba, Johan Verhaghe, Pierre Gambembo) pour l'accompagnement technique du processus de l'étude ainsi que la Cellule d'Appui Technique (CAT) du Ministère de l'EPSP (Valère Munsya, Néné Fwamba, Deo Gracias Nendumba et Nicole Fulakio) pour l'organisation technique, administrative et logistique de l'étude.

Nos remerciements s'adressent également à l'Institut Supérieur des Sciences de la Population (ISSP) de l'Université de Ouagadougou pour la coordination de l'étude, à travers son équipe de recherche conduite par Dr. Jean-François Kobiané (Coordonnateur de la recherche) et composée de M. Idrissa Kaboré (Représentant permanent du coordonnateur en RDC), Dr. Bilampoa Gnoumou/Thiombiano, Dr. Bonayi Dabiré, M. Tinga Sinaré, M. Simon Makaya, Dr. Abdoulaye Ouédraogo, Mme Maria Sombugma, M. Michel Kaboré, M. Sié Somé, Pr. Barthélémy Kalambayi, M. Dramane Boly, M. Alexis Loyé, M. Valérien Kafira, Mme Marie-Fidèle Kashimwabi, M. Adrien Manzanza ainsi que les superviseurs et enquêteurs de terrain et les opérateurs de saisie pour leur contribution à la réalisation de cette importante recherche pour la RD Congo.

Nous exprimons notre gratitude à Max Gasteen et Mukund Lad dont les commentaires ont contribué à l'enrichissement du rapport final.

Enfin, que l'ensemble des acteurs tant au niveau central (PTF, Coordinations nationales, services centraux des ministères en charge de l'éducation, etc.) qu'au niveau local (Gouvernorats, PROVED et Sous-PROVED, Représentants du Ministère des Affaires sociales, Organisations de la société civile, média) ainsi que les populations de l'ensemble des zones sélectionnées trouvent ici la marque de notre reconnaissance et de notre gratitude. En effet, sans leur adhésion au bien fondé de l'étude et leur hospitalité à l'égard des nombreux enquêteurs et superviseurs déployés sur le terrain et leur disponibilité à participer activement aux entretiens, il n'aurait pas été possible de réunir la masse d'informations qui ont rendu possible l'enquête. 


\section{Sommaire}

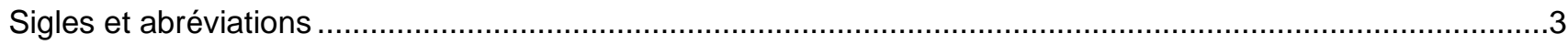

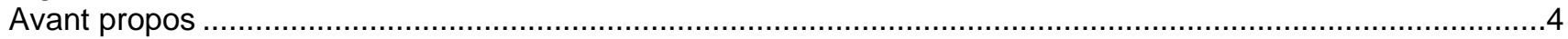

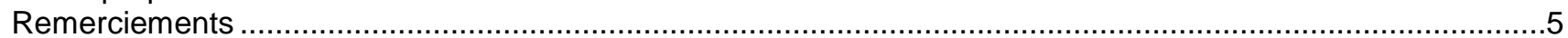

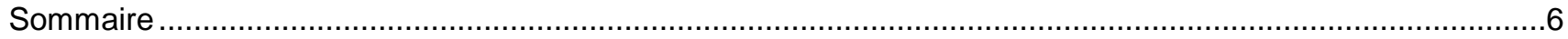

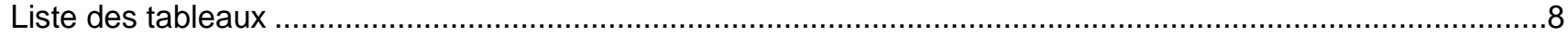

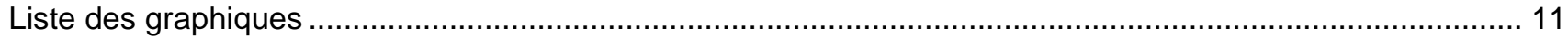

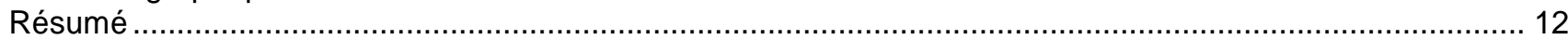

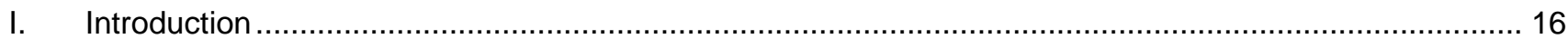

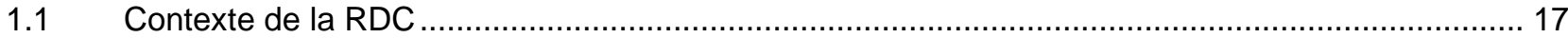

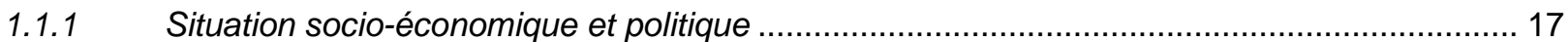

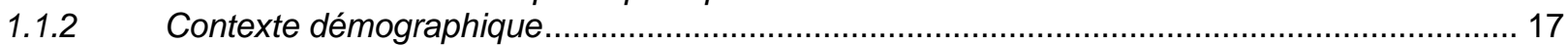

1.1.3 Le système éducatif congolais : politique et stratégies éducatives .....................................18

1.2 Approche globale de l'analyse des EADE : les cinq dimensions de l'exclusion ............................ 19

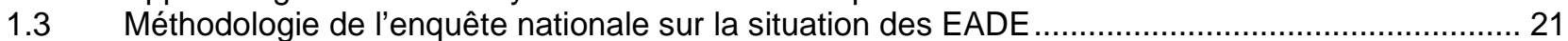

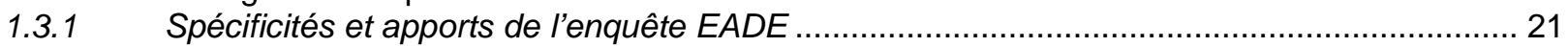

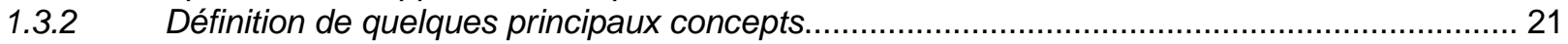

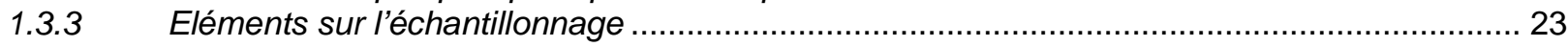

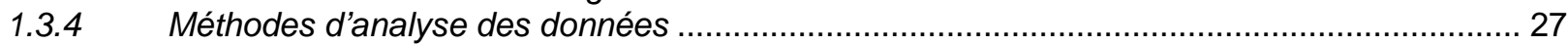

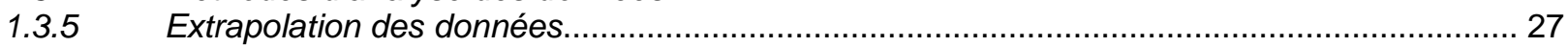

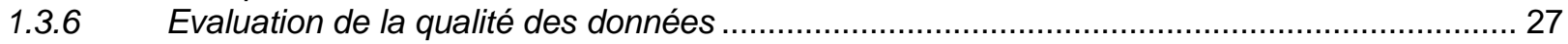

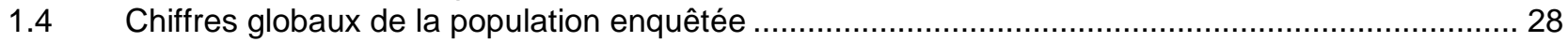

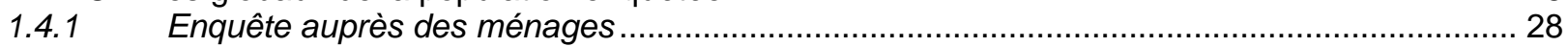

1.4.2 Comparaison des échantillons et des taux couvertures avec les enquêtes EDS (2007) et

$\operatorname{MICS}(2010)$

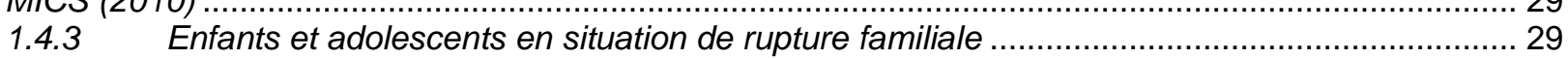

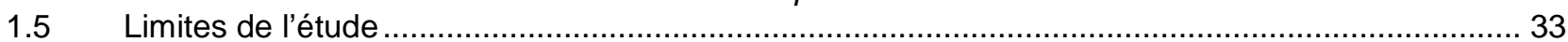

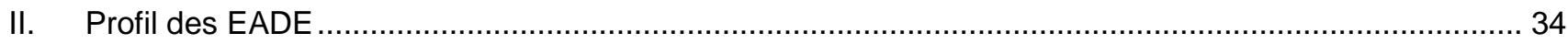

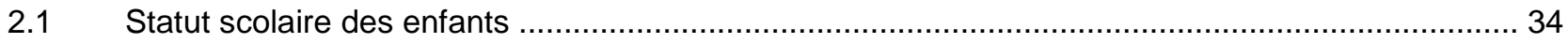

2.1.1 Distribution des enfants et adolescents par statut scolaire selon l'âge et le sexe................... 34

2.1.2 Distribution des enfants et adolescents par statut scolaire selon le milieu de résidence........ 36

2.1.3 Statut scolaire des enfants et adolescents dans les structures et dans la rue ......................41

2.2 Profil des enfants en dehors de l'école d'âges du pré-primaire (Dimension 1) .............................42

2.2.1 Sexe de l'enfant et répartition spatiale des enfants en dehors de l'école de 3-5 ans............ 42

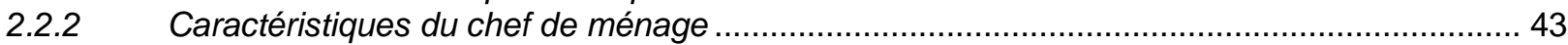

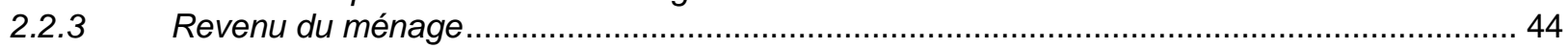

2.3 Profil des EADE d'âges du primaire et du secondaire (Dimensions 2 et 3) ...............................45

2.3.1 Sexe de l'enfant et répartition spatiale des EADE de 6-11 ans et de 12-13 ans.................45

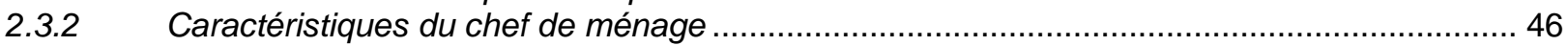

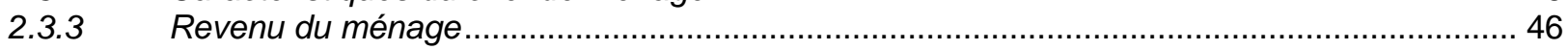

2.3.4 Typologie des enfants et adolescents en dehors de l'école ...........................................47

2.4 Profil des enfants et adolescents à risque d'abandonner (Dimension 4 et 5) ..............................47

2.4.1 Estimation du risque d'abandonner à partir des taux d'abandon déjà réalisés .............................48

2.4.2 Risque d'abandonner au cours $d u$ cycle................................................................. 50

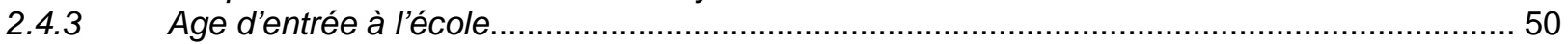

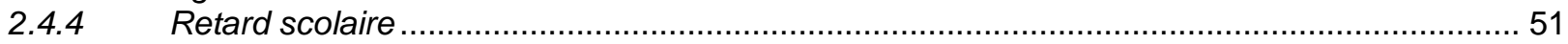

2.4.5 Phénomène des absences au cours de l'année scolaire 2010-2011 ....................................52

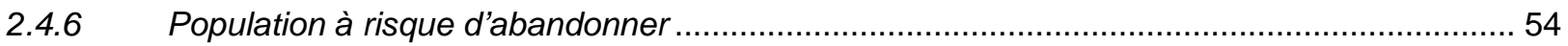

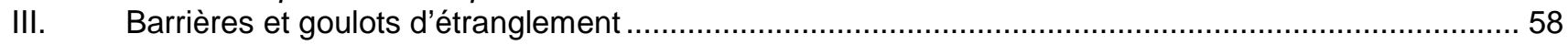

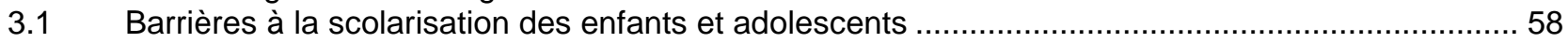

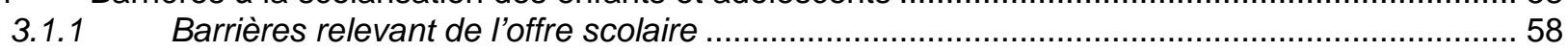

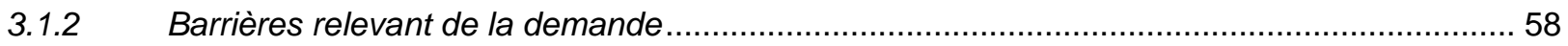

3.2 Déterminants de l'exclusion scolaire des enfants et des adolescents en RDC ...........................60

3.2.1 Déterminants socio-culturels de la demande et environnement familial .............................61

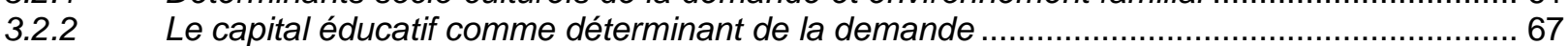

3.2.3 Déterminants économiques de la demande scolaire ...................................................68

3.2.4 Enfants en dehors de l'école et impliqués dans le travail ................................................ 76

3.2.5 Déterminants ou barrières relevant de l'offre .......................................................... 80

3.2.6 Déterminants au niveau politique, gouvernance et financement..................................... 89 


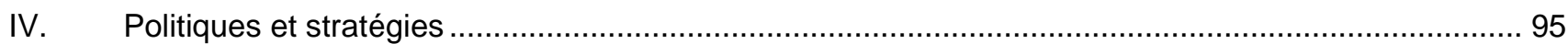

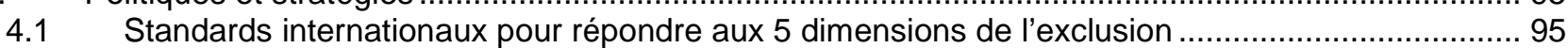

4.2 L'éducative inclusive dans les documents de politique en RDC .............................................. 96

4.3 Politiques et stratégies du côté de la demande socio-culturelle ......................................................96

4.4 Politiques du côté de la demande économique..................................................................... 98

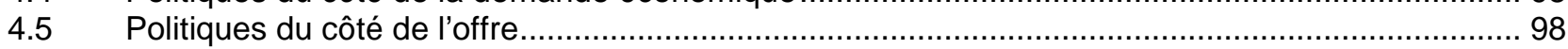

4.6 Au niveau politique, de la gestion et de la gouvernance ....................................................... 99

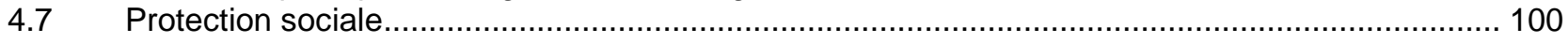

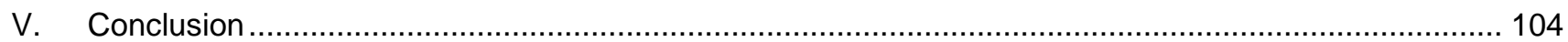

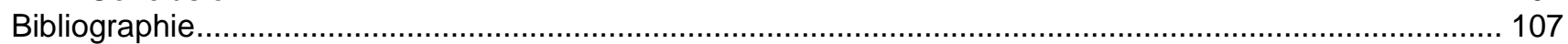

Annexe 1 : Les erreurs de sondage ............................................................................................. 111

Annexe 2 : Formule de calcul de l'intervalle de confiance de la différence de deux proportions ................... 1117

Annexe 3 : Notes sur la régression logistique et la hiérarchisation des déterminants de l'exclusion scolaire 118

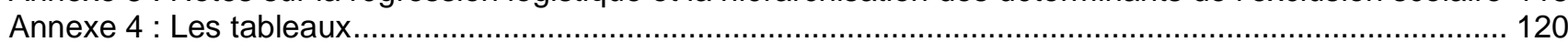

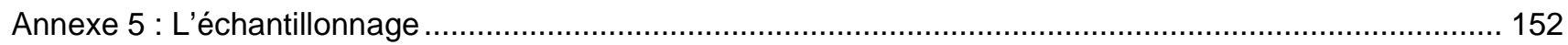




\section{Liste des tableaux}

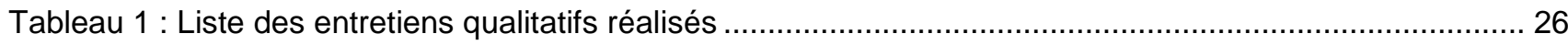

Tableau 2 : Effectif de ménages, nombre d'interviews individuelles et taux de réponse selon le milieu de résidence (Résultats non pondérés), RDC 2012 ........................................................................ 28

Tableau 3 : Taux de couverture (en \%) des enquêtes MICS et EDS de quelques pays ............................... 29

Tableau 4 : Effectif de ménages et taux de réponse selon le milieu de résidence EDS-RDC 2007, MICS-RDC 2010 et EADE-RDC 2012 ....................................................................................... 29

Tableau 5 : Récapitulatif des effectifs de personnes recensées dans la rue et dans les structures d'accueil par catégorie.

Tableau 6 : Répartition (en \%) des personnes de 5-17 ans dans les structures d'accueil et vivant dans la rue, par province.

Tableau 7 : Statut familial des enfants $5-17$ ans dans les structures et dans la rue ..................................... 31

Tableau 8 : Répartition (en \%) des enfants 5-17 ans vivant dans la rue selon le lieu de résidence et le lieu de

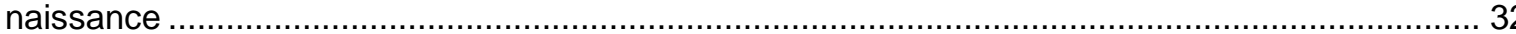

Tableau 9 : Comparaison de la répartition (en \%) selon la situation familiale des enfants vivant dans la rue,

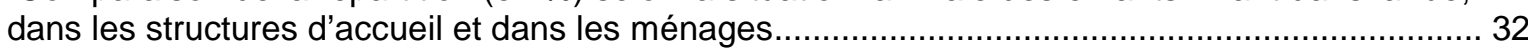

Tableau 10 : Répartition (en \%) de la population scolarisable par groupes d'âges selon le sexe et le milieu de

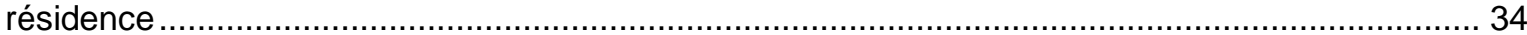

Tableau 11 : Statut scolaire des enfants de 5-17 ans par groupe d'âges et sexe (en \%) ............................ 35

Tableau 12 : Statut scolaire des enfants de 5-17 ans par groupe d'âges et milieu de résidence (en \%) ......... 37

Tableau 13 : Statut scolaire des enfants de 5-17 ans dans les structures d'accueil par groupe d'âges (en \%) 41

Tableau 14 : Statut scolaire des enfants de 5-17 ans de la rue par groupe d'âges (en \%)........................... 42

Tableau 15 : Répartition (en \%) des enfants d'âges du pré-primaire en dehors de l'école par statut scolaire selon le sexe de l'enfant, le milieu de résidence et la province .................................................... 43

Tableau 16 : Répartition (en \%) des enfants d'âges du pré-primaire en dehors de l'école par statut scolaire selon les caractéristiques du chef de ménage

Tableau 17 : Répartition (en \%) des enfants d'âges du pré-primaire en dehors de l'école par statut scolaire selon le revenu mensuel du ménage ........................................................................................ 44

Tableau 18 : Répartition (en \%) des enfants et adolescents en dehors de l'école par statut scolaire et âges de scolarisation (6-11 ans et 12-13 ans) selon le sexe, l'âge, le milieu de résidence et la province .. 45

Tableau 19 : Répartition (en \%) des enfants et adolescents en dehors de l'école par statut scolaire et âges de scolarisation (6-11 ans et 12-13 ans) selon certaines caractéristiques ....................................... 46

Tableau 20 : Répartition (en \%) des enfants et adolescents en dehors de l'école par statut scolaire et âges de scolarisation (6-11 ans et 12-13 ans) selon le revenu mensuel du ménage. ................................ 47

Tableau 21 : Taux d'abandon (en \%) par classe du primaire et du secondaire $1^{\text {er }}$ cycle des enfants et adolescents

Tableau 22 : Proportions (en \%) des enfants et adolescents scolarisés au primaire selon le retard scolaire par sexe et par milieu de résidence ......................................................................................... 51

Tableau 23 : Proportions (en \%) des enfants et adolescents scolarisés au secondaire $1^{\text {er }}$ cycle selon le retard scolaire par sexe et par milieu de résidence............................................................................ 52

Tableau 24 : Proportions d'élèves absents (en \%) par province, sexe et milieu de résidence, année scolaire

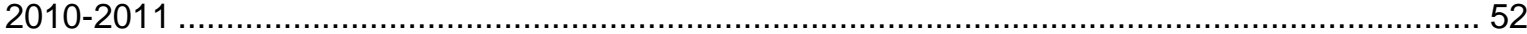

Tableau 25 : Proportions (en \%) des absences selon le niveau et la classe.................................................. 53

Tableau 26 : Durée moyenne d'absence en nombre de semaines par province selon le milieu ..................... 53

Tableau 27 : Profil de la population à risque d'abandonner (en \%) ......................................................... 55

Tableau 28 : Synthèse de l'ampleur des EADE selon les cinq dimensions.................................................56

Tableau 29 : Structure (en \%) des dépenses d'éducation par poste de dépenses (en USD) des ménages pour l'année 2010-2011, par province, milieu de résidence ................................................................ 73

Tableau 30 : Dépense moyenne d'éducation (en USD) des ménages pour l'année scolaire 2010-2011, par

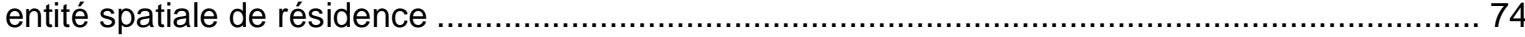

Tableau 31 : Part des dépenses d'éducation dans le revenu annuel estimé des ménages (en USD) pour l'année 2010-2011, par milieu de résidence ............................................................................ 75

Tableau 32 : Proportions (en \%) des EADE d'âges du primaire et du secondaire en dehors de l'école impliqués dans le travail selon les caractéristiques de l'enfant et de leur ménage ......................... 77

Tableau 33 : Proportions (en \%) d'enfants au travail d'âges du primaire et du secondaire impliqués statut scolaire, âge et sexe de l'enfant

Tableau 34 : Proportions (en \%) d'enfants d'âges du primaire et du secondaire impliqués dans le travail par statut scolaire selon certaines caractéristiques du ménage ......................................................... 79

Tableau 35 : Répartition (en \%) des écoles du pré-primaire, du primaire et du secondaire par province de 2006 à 2011 
Tableau 36 : Ratio classe/écoles et ratio élèves/salles de classes du pré-primaire et du primaire par province

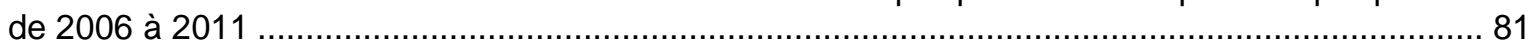

Tableau 37 : Répartition (en \%) des éducateurs, des enseignants du primaire et des enseignants du

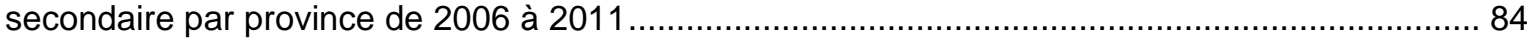

Tableau 38 : Répartition (en \%) des enfants de 6-17 ans ayant un handicap selon le handicap...................... 85

Tableau 39 : Répartition (en \%) des enfants et des adolescents de 7-12 ans scolarisés au primaire en 20102011 selon la dotation en manuels scolaires par milieu de résidence et par sexe......................... 87

Tableau 40 : Répartition (en \%) des enfants et des adolescents de 7-12 ans scolarisés au primaire en 2010-

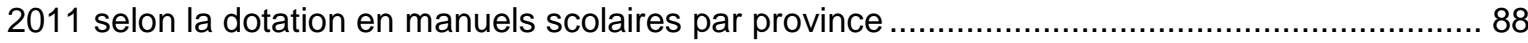

Tableau 41 : Rapport entre les dépenses d'éducation des ménages et le budget de l'éducation (Montants en USD).....

Tableau 42 : Principales politiques pour répondre aux défis des EADE ................................................... 95

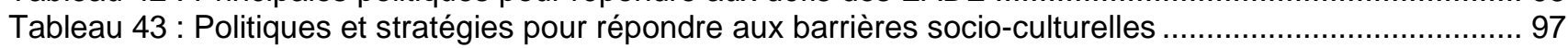

Tableau 44 : Politiques et stratégies pour répondre aux barrières économiques......................................... 98

Tableau 45 : Politiques et stratégies pour répondre aux barrières liées à l'offre.......................................... 99

Tableau 46 : Politiques et stratégies pour répondre aux barrières liées à la gestion et la gouvernance ......... 100

Tableau 47 : Quelques stratégies ou actions visant les groupes vulnérables .............................................. 101

Tableau A1 : Valeur ret répartition de l'échantillon EADE-RDC par strate ................................................. 120

Tableau A2 : Statut scolaire des enfants de 5-17 ans par âge (en \%) ................................................... 121

Tableau A3 : Statut scolaire des enfants de 5-17 ans par âge et sexe (en \%)........................................ 122

Tableau A4 : Statut scolaire des enfants de 5-17 ans par âge et Milieu de résidence (en \%) ..................... 123

Tableau A5 : Distribution (en \%) des enfants en âge scolaire (5-17 ans) selon le statut scolaire par sexe et par

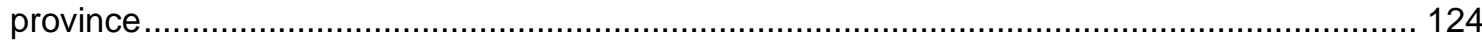

Tableau A6 : Distribution (en \%) des enfants de 6-11 ans selon le statut scolaire par sexe et par province.. 125

Tableau A7 : Distribution (en \%) des enfants et adolescents de 6-13 ans selon la typologie des EADE par sexe, milieu de résidence par dimension...

Tableau A8 : Distribution (en \%) des enfants de 5-17 ans en rupture familiale selon le statut scolaire par province.

Tableau A9 : Répartition des effectifs d'enfants d'âges du pré-primaire en dehors de l'école par statut scolaire selon certaines caractéristiques.....

Tableau A10 : Répartition des effectifs d'enfants d'âges du pré-primaire en dehors de l'école par statut scolaire selon certaines caractéristiques .

Tableau A11 : Statut scolaire (en \%) des enfants de 5-17 ans par classe de revenu mensuel des ménages par milieu de résidence

Tableau A12 : Répartition (en \%) des enfants et adolescents d'âge du primaire et du secondaire en dehors de l'école selon les classes de revenu mensuel des ménages par milieu de résidence............... 131

Tableau A13 : Répartition (en \%) des enfants et adolescents d'âge du primaire et du secondaire en dehors de l'école selon le Statut familial de l'enfant par province

Tableau A14 : Répartition des effectifs d'enfants et d'adolescents d'âges du primaire en dehors de l'école par statut scolaire selon certaines caractéristiques .................................................................... 133

Tableau A15 : Répartition des effectifs d'enfants et d'adolescents d'âges du primaire en dehors de l'école par statut scolaire selon certaines caractéristiques

Tableau A16 : Répartition des effectifs d'enfants et d'adolescents d'âges du secondaire (12-13 ans) en dehors de l'école par statut scolaire selon certaines caractéristiques............................................... 135

Tableau A17 : Répartition des effectifs d'enfants et d'adolescents d'âges du secondaire (12-13 ans) en dehors de l'école par statut scolaire selon certaines caractéristiques................................................ 136

Tableau A18 : Probabilités cumulées (en \%) d'abandonner par sexe et par milieu de résidence .................. 137

Tableau A19 : Probabilités cumulées (en \%) d'abandonner par province.................................................. 137

Tableau A20 : Probabilités cumulées (en \%) d'entrée à l'école par sexe et par milieu ................................. 138

Tableau A21 : Probabilités cumulées (en \%) d'entrée à l'école par province ............................................ 138

Tableau A22 : Répartition (en \%) du statut scolaire des enfants et adolescents scolarisés au primaire selon le retard scolaire par classe du primaire par sexe et par milieu de résidence ............................ 139

Tableau A23 : Proportion (en \%) des enfants et adolescents scolarisés au secondaire $1^{\mathrm{er}}$ cycle selon le retard scolaire par classe du secondaire par sexe et par milieu de résidence .................................. 140

Tableau A24 : Répartition (en \%) du statut scolaire des enfants et adolescents scolarisés au primaire par

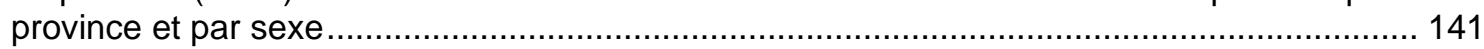

Tableau A25 : Répartition (en \%) de la population de 6-11 ans selon le statut d'exclusion par caractéristique

Tableau A26 : Répartition (en \%) de la population de 12-17 ans selon le statut d'exclusion par caractéristiques

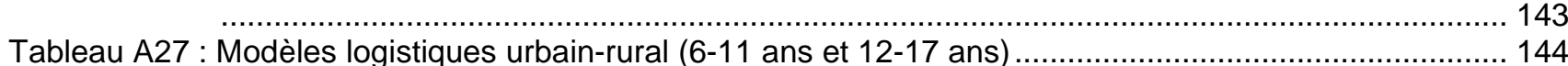

Tableau A28 : Modèles logistiques au niveau province (6-11 ans) ....................................................... 145 
Tableau A29 : Modèles logistiques au niveau province (12-17 ans) ......

Tableau A30 : Hiérarchisation des variables selon le pouvoir explicatif du risque d'être en dehors de l'école au niveau des 6-11 ans et au niveau des 12-17 ans

Tableau A31 : Raisons d'arrêt ou d'abandon scolaire (en \%) et raisons de non inscription à l'école (en \%) des enfants de 6-11 ans et de 12-17 ans....

Tableau A32 : Répartition (en \%) des enfants âgés de 6-17 ans n'ayant jamais été à l'école et ceux déscolarisés selon les raisons de non inscription ou d'arrêt de scolarisation 149

Tableau A33 : Proportions (en \%) des EADE d'âge du primaire et du secondaire en dehors de l'école impliqués dans le travail selon les caractéristiques de l'enfant et de leur ménage 150

Tableau A34 : Taux de couverture (en \%) de l'EADE-RDC 2012 par province 


\section{Liste des graphiques}

Graphique 1 : Illustration des cinq dimensions de l'exclusion (5DE) ...................................................... 20

Graphique 2 : Evolution de la proportion (en \%) des enfants et adolescents en dehors de l'école selon la tranche d'âges

Graphique 3 : Carte des proportions (en \%) et des effectifs des EADE de 5-17 ans par province, EADE-RDC 2012

Graphique 4 : Carte des proportions (en \%) et des effectifs des EADE de 6-11 ans par province, EADE-RDC 2012.

Graphique 5 : Evolution de la proportion (en \%) des enfants de 5-17 ans en dehors de l'école par province.. 39

Graphique 6 : Proportion (en \%) d'enfants de 5-17 ans en dehors de l'école par sexe et selon la province.... 39

Graphique 7 : Participation scolaire par âge et niveau d'études des enfants et adolescents de 5-17 ans en 2012 (en \%)

Graphique 8 : Typologie des enfants et adolescents en dehors de l'école

Graphique 9 : Probabilités cumulées (en \%) d'abandonner par sexe et par milieu de résidence

Graphique 10 : Probabilités cumulées (en \%) d'entrée à l'école par sexe et par milieu de résidence.....

Graphique 11 : Profil scolaire des enfants et adolescents de 5-17 ans en 2012 selon certaines caractéristiques (en \%)

Graphique 12 : Proportion (en \%) d'EADE selon le statut d'orphelin et par groupe d'âge....

Graphique 13 : Proportion (\%) des EADE selon le niveau d'instruction du chef de ménage et par groupe d'âges des enfants

Graphique 14 : Distribution (en \%) des classes de revenu par milieu de résidence .....................................68 68

Graphique 15 : Distribution (en \%) des classes de revenu par province ................................................. 69

Graphique 16 : Proportions (en \%) des enfants et des adolescents de 5-17 ans en dehors de l'école par revenu mensuel du ménage et par sexe .......................................................................... 70

Graphique 17 : Proportions (en \%) des enfants et des adolescents de 5-17 ans en dehors de l'école par revenu mensuel du ménage et par milieu de résidence

Graphique 18 : Profil scolaire et implication dans le travail des enfants et des adolescents de 6-13 ans en fonction de la définition du travail

Graphique 19 : Evolution de la proportion (en \%) des salles de classe du primaire en bon état par province 82

Graphique 20 : Evolution de la proportion (en \%) des salles de classe du secondaire en bon état par province

Graphique 21 : Proportion (en \%) des enfants scolarisables (5-17 ans) en dehors de l'école selon la nature du handicap et par sexe

Graphique 22 : Proportion (en \%) des enfants scolarisables (5-17 ans) en dehors de l'école selon la nature du handicap et par milieu de résidence................................................................................... 86

Graphique 23 : Proportion (en \%) des EADE selon la distance à l'établissement le plus proche ................... 88 


\section{Résumé}

L'UNICEF et l'Institut de Statistique de I'UNESCO ont lancé en 2010 une initiative mondiale sur les Enfants et Adolescents en Dehors de l'Ecole (EADE), en vue d'améliorer le système d'information statistique et d'analyse sur les EADE et examiner les facteurs d'exclusion de l'école. En RDC, cette étude sur les EADE a été réalisée en deux phases : la première phase a porté sur un état des lieux, ayant consisté en une revue documentaire des travaux existants sur l'éducation et l'analyse des sources de données secondaires (statistiques administratives et enquêtes auprès des ménages). Sur la base des enseignements tirés de cet état des lieux, la deuxième phase a consisté à la réalisation d'une enquête nationale spécifique sur la question des EADE. La recherche sur les EADE cherchait à répondre aux questions suivantes : "Combien sont les EADE ? ; "Où sont-ils ? ; «Qui sont-ils »; "Pourquoi sont-ils en dehors de l'école ? »; "Quels sont l'effectivité et l'impact des politiques et stratégies dans les secteurs de l'éducation et de la protection sociale ? " et "Quelles politiques et stratégies pour permettre la participation de tous les enfants et adolescents à l'école ? ».

\section{Combien sont-ils?}

La proportion des 5-17 ans en dehors de l'école est estimée à 28,9\% par l'EADE 2012, soit en termes absolus, 7375876 enfants et adolescents. Parmi ces enfants en dehors de l'école, les filles représentent plus de la moitié soit 3892464 (52,7\%). C'est aussi chez les filles que l'ampleur du phénomène des enfants et adolescents en dehors de l'école est la plus forte : $31,7 \%$ chez les filles contre $26,5 \%$ chez les garçons. Cet effectif se décline ainsi qu'il suit en fonction des différents sous-groupes d'âges : 2144195 pour les 5 ans (78,8\% de la population de 5 ans) ; 3509251 pour les $6-11$ ans (26,8\% de la population de $6-11$ ans); 513168 pour les $12-13$ ans (représentant 13,1\% de la population de 12-13 ans) et 1209262 pour les 14-17 ans (représentant $21,2 \%$ des $14-17$ ans). C'est donc dans la tranche d'âge de la scolarisation obligatoire (611 ans) que l'effectif des EADE est le plus élevé, soit 47,6\% des EADE. Par ailleurs, les EADE âgés de 6-7 ans sont au nombre de 2101871 , soit $59,9 \%$ des EADE de 6-11 ans, pointant ainsi du doigt le problème de l'entrée tardive à l'école.

L'évolution de la proportion des enfants en dehors de l'école révèle que le phénomène est en baisse régulière depuis 2007, passant de 38,5\% en 2007 (EDS 2007), à 32,5\% en 2010 (MICS 2010) et $28,9 \%$ en 2012 (EADE 2012). En cinq ans (2007-2012), la baisse relative de la proportion des EADE a été la plus importante dans la tranche d'âge des $12-13$ ans (33,5\% de baisse), suivie de la tranche d'âges des $6-11$ ans (31,5\%), de celle des 14-17 ans (27,6\%). Elle a été la plus faible chez les 5 ans $(14,2 \%)$.

\section{Où sont-ils ?}

La proportion d'EADE est plus forte en milieu rural $(33,4 \%)$ qu'en milieu urbain $(20,0 \%)$. En termes absolus, c'est aussi en milieu rural qu'on retrouve le plus grand nombre des EADE, soit 5694525 d'EADE en milieu rural contre 1681391 en milieu urbain.

L'analyse spatiale de l'ampleur du phénomène révèle que c'est au Nord Kivu qu'il y a proportionnellement plus d'enfants d'âges scolaires en dehors de l'école, soit $43,9 \%$, suivis du Katanga (34,8\%), du Kassaï occidental $(32,4 \%)$, de la province orientale (32,2 \%), du Sud-Kivu (30,3\%) et du Kassaï oriental $(29,3 \%)$. En termes absolus, c'est le Katanga qui vient en tête avec 1334876 enfants de 5-17 ans en dehors de l'école, suivi de la Province orientale (1 039 858), du Nord Kivu (994 366) et de l'Equateur (726 194). C'est donc dans les provinces à forte production minière et celles où les conflits sont récurrents, que l'ampleur du phénomène est la plus forte.

\section{Qui sont-ils?}

L'examen du profil des EADE montre :

- Que les enfants d'âges du pré-primaire en dehors de l'école sont davantage dans les ménages à faible revenu $(56,1 \%)$.

- Que les EADE de 6-11 ans se retrouvent plus dans les ménages les plus pauvres (64,3\%) et dans les ménages dont le chef n'a aucun niveau d'instruction (65,3\%).

- Les EADE de 12-13 ans sont davantage des filles (60,4\%), se retrouvent plus dans les ménages les plus pauvres $(64,9 \%)$ et dans les ménages dont le chef de ménage n'a aucun niveau d'instruction $(46,5 \%)$. 
- Les EADE de 14-17 ans sont en majorité des filles (63,8\%), se retrouvent plus dans les ménages les plus pauvres $(60,2 \%)$ et dans les ménages dont le chef de ménage n'a aucun niveau d'instruction $(50,8 \%)$.

Par ailleurs, l'analyse du cumul des inégalités révèle que pour les filles des ménages les plus pauvres, dont le chef de ménage n'a aucun niveau d'instruction et résidant en milieu rural, la proportion des EADE est 45,0 \% contre $37,6 \%$ pour les garçons dans la même situation. Ceci donne une idée de l'ampleur des efforts qu'il y aura à faire pour ramener tous les enfants et adolescents de la RDC à l'école.

\section{Pourquoi sont-ils en dehors de l'école?}

Au niveau de la demande scolaire, les déterminants de l'exclusion scolaire sont d'ordres socio-culturels et économiques. En effet, les résultats des analyses révèlent des inégalités de genre dans l'éducation. II ressort des analyses que si les efforts déployés par l'État et ses partenaires dans la scolarisation des enfants ces dernières années ont permis de réduire de façon significative les inégalités d'accès à l'école entre garçons et filles de nos jours, des inégalités de genre existent toujours au niveau secondaire, surtout en milieu rural en défaveur des filles. Les logiques matrimoniales familiales et les grossesses précoces expliquent en partie cette déperdition scolaire plus importante chez les filles aux âges du secondaire. II ressort des résultats que la proportion de filles de 12-17 ans scolarisées et qui sont en union est de 0,08\%, alors que la proportion de filles de 12-17 en dehors de l'école qui sont en union au moment de l'enquête est de 9,0\%. Même si l'entrée en union peut intervenir après la sortie de l'école et peut ne pas être nécessairement la cause de l'abandon, cette forte proportion des filles EADE en union fait penser à un effet des mariages précoces comme cause de déperdition scolaire chez les filles. Par ailleurs, les chiffres sur les raisons d'abandon scolaire des filles révèlent que le mariage a été cité dans $6,3 \%$ des cas et la grossesse citée dans 5,2 \% des cas d'arrêt de scolarisation.

La survie des parents constitue aussi un des déterminants de la scolarisation des enfants. Quel que soit le milieu de résidence, le décès d'un parent ou des deux parents est un facteur d'exclusion scolaire. Ces résultats enregistrés dans les analyses quantitatives sont également confirmés dans les entretiens qualitatifs où certains acteurs soulignent la faible scolarisation des filles par rapport aux garçons au niveau secondaire et supérieur de l'enseignement, ainsi que les difficultés de scolarisation des orphelins.

Le confiage des enfants, une pratique courante en RDC, comme dans d'autres pays africains, est aussi un des déterminants de l'exclusion scolaire. Les enfants confiés (apparentés ou non au chef de ménage) ont un risque plus grand de se retrouver en dehors de l'école que les enfants biologiques du chef de ménage. Les points de vue donnés par les acteurs (adultes, enfants et adolescents) sur la question, montrent que les enfants confiés sont souvent utilisés pour accomplir de multiples tâches dans leur ménage d'accueil, ce qui diminue leurs chances de scolarisation.

D'ailleurs, selon les propos des acteurs, le principe du respect de l'opinion de l'enfant est difficilement admis dans la société congolaise comme dans la plupart des sociétés africaines où seuls les adultes ont le droit de prendre des décisions. Cette perception des parents sur les droits des enfants constitue sans doute un facteur pouvant influer sur la décision des parents à investir dans la scolarisation des enfants et donc accroître le risque d'exclusion scolaire.

Le capital éducatif des parents est aussi un des déterminants de la scolarisation des enfants. Les résultats des analyses quantitatives montrent que, quel que soit le milieu de résidence, le niveau d'instruction du chef de ménage est l'un des principaux déterminants de l'exclusion scolaire. Les enfants vivant dans les ménages où le chef a le niveau d'instruction secondaire ou plus, ont moins de risque de se retrouver en dehors de l'école que ceux dont le chef de ménage n'a aucun niveau d'instruction.

Sur le plan économique, le revenu du ménage détermine la fréquentation scolaire des enfants et des adolescents. Que ce soit en milieu urbain ou en milieu rural, le faible revenu du ménage constitue une importante barrière à la scolarisation des enfants tant au niveau primaire que secondaire. La proportion d'EADE décroit de manière régulière et nette lorsque le revenu du ménage augmente : au niveau national, on passe d'une proportion d'EADE de 48,9\% dans les ménages à moins de 50 USD par mois, à 1,9\% dans les ménages à plus de 500 USD par mois. Les estimations faites par l'enquête EADE-RDC révèlent qu'un ménage en RDC a consacré en 2010-2011 plus du dixième (11,2 \%) de son revenu annuel aux dépenses d'éducation des enfants. Cette barrière est la principale raison invoquée par les ménages pour justifier la non inscription à l'école ou l'abandon scolaire des enfants, elle est aussi confirmée par les propos des acteurs interviewés lors des entretiens qualitatifs. 
La situation des groupes vulnérables restent encore préoccupante, puisqu'ils sont encore en grande majorité en dehors de l'école : $54,5 \%$ des enfants et adolescents vivant avec un handicap ; $32,2 \%$ des orphelins ; $36,6 \%$ des enfants et adolescents dans les ménages les plus pauvres.

Au niveau de l'offre scolaire, bien qu'on note globalement une augmentation de l'offre scolaire ces dernières années à tous les niveaux de l'enseignement, il existe des disparités entre provinces et entre milieux urbain et rural quant à la disponibilité et à la répartition des infrastructures scolaires. Au niveau du pré-primaire, une grande part des écoles maternelles se trouve en milieu urbain principalement dans la ville province de Kinshasa. Au niveau du primaire et du secondaire, le nombre d'écoles a considérablement augmenté ces dernières années, mais les écoles primaires et surtout secondaires sont encore inégalement réparties entre les provinces. Pourtant les analyses montrent que la grande distance à l'école constitue un des principaux déterminants de l'exclusion scolaire en milieu rural. De plus, d'une manière générale, au niveau de l'ensemble du pays, à l'exception de quelques provinces, on note une dégradation de la qualité des infrastructures ces dernières années tant au niveau du primaire que du secondaire.

En ce qui concerne l'offre en personnel enseignant, la répartition des enseignants du primaire et du secondaire suit celle des écoles (c'est dans les provinces où il y a le plus d'écoles que l'on trouve le plus d'enseignants). Toutefois, cette offre scolaire est inadaptée aux besoins de certains groupes spécifiques tels que les enfants et adolescents vivant avec un handicap.

Au niveau politique, il s'agit notamment du faible financement de l'éducation par l'Etat, qui contraint les ménages à consacrer une part importante de leur revenu annuel aux dépenses d'éducation (14 \% en milieu urbain et $7 \%$ en milieu rural), et aussi de la faiblesse de la capacité institutionnelle et humaine du secteur de l'éducation. Ce problème de capacité institutionnelle et humaine se pose au niveau du fonctionnement des comités de gestion des écoles, au niveau de la gestion des ressources humaines et au niveau de la coordination et de la gestion. Globalement les acteurs soulignent un disfonctionnement des comités de gestion (faible capacité de gestion, manque de concertation et de transparence dans la gestion des fonds), et une faible communication entre les différents acteurs. Pourtant, la réussite des politiques et stratégies éducatives nécessite une bonne coordination des actions et une réelle concertation entre les différents acteurs du système éducatif.

En ce qui concerne la gestion des ressources humaines du secteur de l'éducation, les acteurs soulignent plusieurs difficultés. II s'agit notamment de l'insuffisance et de l'irrégularité des salaires des enseignants, de l'absence d'un système de retraite pour les enseignants, du manque de formation de certains enseignants, du non renouvellement du personnel enseignant, etc. Ces difficultés ont certainement des effets néfastes sur la qualité de l'enseignement et contribuent à l'exclusion scolaire des enfants.

\section{Quelles politiques et stratégies?}

A la suite des barrières et goulots d'étranglement identifiés, des politiques, et stratégies sont proposées en vue d'éliminer les barrières à la scolarisation des enfants et adolescents en dehors de l'école. Au niveau socio-culturel, un certain nombre de stratégies ou d'actions ont été proposées pour répondre aux problèmes des représentations sociales défavorables aux filles, aux mariages et grossesses précoces, aux perceptions sur les droits et la place de l'enfant dans la société congolaise, aux effets parfois néfastes du confiage des enfants et aux perceptions des communautés sur les potentialités économiques de leur environnement de vie. En ce qui concerne la scolarisation des filles, il est proposé d'intensifier les campagnes de sensibilisation, pour la scolarisation des filles, particulièrement au niveau du secondaire, de mettre en place des programmes d'octroi de bourses et envisager comme cela a été expérimenté ailleurs, des dotations incitatives aux ménages. Pour éliminer le phénomène des mariages précoces, il est suggéré de faire appliquer la législation en matière d'âge légal au mariage et pour les grossesses précoces, d'intensifier les cours d'éducation en santé sexuelle et reproductive.

En ce qui concerne le faible niveau d'instruction et d'alphabétisation des parents et des perceptions sur les droits des enfants, il est suggéré de mettre en place des campagnes de sensibilisation orientées vers les chefs de ménage non instruits/non alphabétisés, et de renforcer les programmes d'alphabétisation, les capacités socio-économiques des jeunes et des adultes, et augmenter la connaissance des acteurs sur les lois relatives à la protection et au droit de l'enfant.

Pour ce qui est des effets néfastes du confiage, il est proposé de développer les programmes d'éducation parentale afin de rappeler aux parents leurs obligations envers leurs enfants. Enfin, pour accroître les capacités et les ressources des communautés, il est proposé de mettre en place des approches multisectorielles pour renforcer les capacités institutionnelles et communautaires d'autonomisation en matière 
de mobilisation de ressources financières pour amener les populations vulnérables à réaliser leurs potentialités économiques dans leur environnement immédiat.

Au niveau des barrières économiques, le rôle important joué par la pauvreté comme barrière à la scolarisation des enfants conduit à réaffirmer l'urgence qu'il y a d'accélérer et de rendre effective la réduction des frais scolaires. En ce qui concerne le travail des enfants, particulièrement le travail dangereux, il s'agira de renforcer les lois sur la protection et les droits des enfants et adolescents et renforcer les programmes d'éducation parentale.

Au niveau de l'offre, ressort la nécessité d'accroître substantiellement les infrastructures, d'accroître en nombre et en qualité le personnel enseignant (particulièrement la proportion des femmes enseignantes pour accroître la rétention des filles à l'école), améliorer de manière substantielle la rémunération des enseignants tout en leur assurant un plan de carrière adéquat, mettre en place des approches pédagogiques spécifiques pour chaque catégorie d'enfant vivant avec un handicap, renforcer les initiatives tendant à rendre possible la scolarisation des enfants de la rue et renforcer les initiatives tendant à permettre la scolarisation des enfants dans les sites d'orpaillage.

Au plan politique et de la gouvernance, il est proposé d'accroître de manière substantielle les ressources de l'Etat allouées à l'éducation, de développer un partenariat Etat/privé, avec des fora annuels en vue de prévenir de l'exploitation économique des enfants et des jeunes, d'instituer un " fonds de compensation communautaire " dans les zones à forte production industrielle, d'étudier les options possibles pour une augmentation progressive de la participation à l'enseignement secondaire pour les filles et les garçons, d'améliorer le système de pilotage (communication entre acteurs au niveau central mais aussi entre niveau central et niveau local), de mettre en place des mécanismes au niveau provincial en vue d'une sensibilisation et d'information sur les potentialités économiques et intellectuelles des provinces. 


\section{Introduction}

Les rapports sur le suivi des Objectifs du Millénaire pour le Développement (OMD) de ces dernières années révèlent que l'un des objectifs pour lesquels des progrès notables ont été observés dans les pays en développement et notamment en Afrique est l'objectif 2, celui de la réalisation de l'éducation primaire universelle. Toutefois, malgré ces progrès, plusieurs enfants et adolescents, notamment des couches sociales les plus vulnérables et des zones rurales demeurent encore en dehors du système éducatif. Or, au fur et à mesure que les pays s'acheminent vers l'atteinte des objectifs de l'EPT, plus les efforts à déployer pour atteindre les exclus du système éducatif seront importants.

Le rapport mondial de suivi de l'EPT de 2012 révèle, sur la base de l'Indice de Développement de l'Éducation (indice composite des progrès de l'EPT), que sur les 20 pays qui sont à la traîne (IDE $<0,80$ ), 12 sont situés en Afrique subsaharienne (UNESCO, 2012). Au-delà de cette situation d'ensemble du continent africain, les niveaux de scolarisation révèlent des disparités non seulement d'un pays à un autre, mais aussi à l'intérieur d'un même pays, entre zones géographiques, d'une part et entre différentes catégories sociales, d'autre part. L'objectif 2 du Cadre d'action du Forum de Dakar met en exergue la nécessité d'accorder un intérêt croissant aux groupes vulnérables ou marginalisés, notamment de " faire en sorte que, d'ici 2015, tous les enfants, en particulier les filles, les enfants en difficulté et ceux qui appartiennent à des minorités ethniques, aient la possibilité d'accéder à un enseignement primaire obligatoire et gratuit, de qualité et de le suivre jusqu'à son terme " (UNESCO, 2000, p. 15). En vue de répondre à ces défis et réaliser le droit à l'éducation pour tous les enfants, I'UNICEF et l'Institut de Statistique de I'UNESCO (ISU), ont lancé en début 2010 une initiative mondiale sur les Enfants et Adolescents en Dehors de l'Ecole (EADE), dans laquelle 26 pays sont engagés. L'objectif de cette initiative est d'améliorer le système d'information statistique et d'analyse sur les EADE et examiner les facteurs d'exclusion de l'école ainsi que les politiques d'amélioration de la participation scolaire.

En RDC, l'étude sur les EADE est menée sous l'égide du Ministère de l'Enseignement Primaire, Secondaire et Professionnel (Min-EPSP), avec le soutien financier de DFID et de I'UNICEF, l'appui technique de I'ISU/UNESCO et de I'UNICEF, et l'exécution technique de l'ISSP/Université de Ouagadougou. L'étude s'est réalisée en deux phases : la première phase a porté sur l'état des lieux (MEPSP-UNICEF-ISSP, 2011) qui a consisté en une revue documentaire des travaux existants sur la question et l'analyse des sources de données secondaires (statistiques administratives et enquêtes auprès des ménages), et qui a aboutit à la production d'un rapport en juillet 2011. Tirant les leçons de cet état des lieux, la deuxième phase a consisté à la réalisation d'une enquête nationale spécifique sur la question des EADE.

Le présent rapport final de recherche sur les EADE en RDC présente les résultats de l'enquête nationale spécifique sur les EADE, tout en répondant aux questions de recherche suivantes: "Combien sont les EADE ? "; « Où sont-ils ? » ; "Qui sont-ils »; "Pourquoi sont-ils en dehors de l'école ? » ; "Quels sont l'effectivité et l'impact des politiques et stratégies dans les secteurs de l'éducation et de la protection sociale ? ». S'articulant autour de ces questions de recherche, le rapport est structuré en cinq (5) chapitres :

- Chapitre 1 (Introduction) : présente successivement le contexte du pays, le système éducatif et les aspects méthodologiques de l'étude;

- Chapitre 2 (Profil des enfants et adolescents en dehors de l'école) : rappelle tout d'abord quelques chiffres globaux sur la population enquêtée, présente ensuite le profil scolaire des enfants et adolescents et fait ensuite une description des caractéristiques démographiques, sociales et économiques des EADE par rapport aux cinq (5) dimensions de l'exclusion;

- Chapitre 3 (Barrières et goulots d'étranglement) : présente les principaux facteurs, tant du côté de l'offre que de la demande, qui contribuent à exclure les enfants du système éducatif, ainsi que tous les apports de l'enquête qualitative quant aux opinions et perceptions sur les inégalités en matière d'éducation ainsi que les causes de non scolarisation des enfants ;

- Chapitre 4 (Politiques et stratégies) : présente quelques éléments de réflexion en termes d'implications politiques pour surmonter les obstacles à la scolarisation de tous les enfants et adolescents;

- Chapitre 5 (Conclusion) : reprends les principaux résultats de la recherche ainsi que ses implications politiques. 


\subsection{Contexte de la RDC}

\subsubsection{Situation socio-économique et politique}

Depuis la fin des années 70, l'économie de la RDC connaît une crise multiforme qui s'est amplifiée au début des années 90 avec l'instabilité politique du pays. Par exemple, entre 1990 et 2000, le PIB a enregistré une baisse cumulée de plus de $43 \%$. Cette situation a entraîné une baisse de près de $60 \%$ du revenu national moyen par tête. Malgré les importantes ressources naturelles dont dispose le pays, l'incidence de la pauvreté reste énorme, comme l'indiquent les analyses effectuées dans le cadre du Document de Stratégie de Croissance et de Réduction de la Pauvreté (DSCRP) publié en 2006 et dans d'autres travaux recourant notamment aux données de l'enquête 1-2-3 de 2004-2005 (Banque Mondiale, 2006 ; Moummi, 2010) : l'incidence de la pauvreté (proportion de personnes en dessous du seuil de pauvreté) en 2005 est estimée à $69,2 \%$ au niveau national, et est plus forte en milieu rural $(71,7 \%)$ qu'en milieu urbain $(58,3 \%)$ (Moummi, 2010, p. 12).

La RDC se classe ainsi parmi les pays les plus pauvres du monde avec un PIB par tête d'environ 120 dollars USD, soit six fois inférieur à la moyenne africaine (RDC, 2009). Cette situation est aggravée par le poids de la dette extérieure, qui en dépit de l'assistance obtenue dans le cadre de l'initiative PPTE, reste un fardeau pour les finances publiques ainsi que pour la balance des paiements. Par exemple, en 2007, l'encours de la dette extérieure était évalué à plus de 10 millions de dollars USD et son service représentait $6,9 \%$ des exportations et $12,9 \%$ des recettes fiscales, soit 480 millions de dollars USD. Au cours de la période 2002-08, la croissance et l'inflation ont augmenté respectivement de $6 \%$ et de 15,9\% par an (RDC, 2010).

Toutefois, la reprise de la coopération avec l'appui des partenaires extérieures (FMI, BM, UE, BAD) et les efforts mis en œuvre par le gouvernement en vue d'instaurer la démocratie et la paix dans le pays, ont permis une reprise de l'économie. Mais, bien qu'importants, les résultats restent insuffisants et précaires au regard des défis énormes qui se posent et en raison des potentialités en ressources nationales dont dispose la RDC.

La situation économique du pays a, de toute évidence, des effets négatifs sur le développement du système éducatif en général. Cela a entraîné notamment la stagnation, voire l'amenuisement des ressources allouées à l'éducation pourtant indispensables pour la mise en œuvre des objectifs stratégiques pour le développement du secteur éducatif de la RDC. On peut toutefois noter un signe positif en termes de mobilisation de ressources qui devrait profiter aux secteurs sociaux, notamment l'éducation: en effet, les progrès économiques récents (croissance du PIB de plus de $7 \%$ en 2010 et du taux d'inflation en dessous des $10 \%$ ), et l'atteinte du point d'achèvement de l'initiative en faveur des pays pauvres très endettés (PPTE) ont eu pour résultat un allègement de la dette du pays de 12,3 milliards de dollars par les conseils d'administration du FMI et de l'Association internationale de développement (IDA) de la Banque mondiale à la mi-2010'.

\subsubsection{Contexte démographique}

\section{a) Les sources de données sur la population}

Les sources de données classiques sur la population sont les recensements généraux de la population, les enquêtes socio-démographiques et l'état civil. Le recensement général de la population est une source exhaustive d'information sur la population, permettant de produire des indicateurs socio-démographiques et économiques à des niveaux géographiques très fins. Toutefois, contrairement à plusieurs pays africains qui comptent de nos jours plusieurs recensements généraux de la population, la RDC n'en a réalisé qu'un seul datant de 1984. Les résultats de projections utilisés dans la planification des besoins sociaux, notamment celles des Nations Unies et de l'Institut National de la Statistique (INS) partent des données du recensement de 1984. Bien que très utiles, elles sont aussi sujettes à caution dans la mesure où toute projection est basée sur des hypothèses qu'il faut d'ailleurs réviser périodiquement à la lueur des résultats des enquêtes démographiques.

L'état civil n'est pas non plus de nos jours une source de données fiable, contrairement à la période coloniale. A cette époque, l'état civil et le registre de population fonctionnaient assez bien et étaient mis à jour (Ngondo, 2001 ; Metela, 2010). Comme dans beaucoup de pays africains, l'état civil reste peu développé et sa couverture se limite essentiellement au milieu urbain.

\footnotetext{
${ }^{1}$ Cf. dossier spécial sur la RDC dans Afrique magazine (AM) de juillet 2011 (p. 90-91).
} 
Ce sont surtout les enquêtes auprès des ménages qui fournissent régulièrement des données sur la population. On peut citer, entre autres, les enquêtes MICS (1995, 2001 et 2010), l'EDS (2007) et l'enquête 12-3 (2004-2005). Toutefois, ces sources d'informations ne permettent pas souvent de calculer des indicateurs au niveau des petites entités administratives, afin de mettre en évidence leurs spécificités. En revanche, diverses enquêtes sociodémographiques très localisées (monographies) sont réalisées, mais leur portée étant limitée, elles ne peuvent être généralisées à l'ensemble du pays.

Enfin, la diversité des sources de données sur la population explique la multiplicité et la discordance des indicateurs socio-démographiques se référant à une même période.

\section{b) Caractéristiques de la population congolaise}

Le manque de données de recensement récent, rend difficile l'estimation de la population congolaise. Aussi, l'estimation de la population varie selon les sources entre 64 et 68 millions d'habitants pour les années 2009 et 2010 : 67 millions d'habitants en 2009 selon le Programme National Multisectoriel de Lutte contre le Sida de la RDC (PNMLS, 2009) ; 64,420 millions d'habitants en 2010 d'après le Ministère de la Santé Publique et 67,827 millions d'habitants en 2010 d'après OIM-RDC. La population serait passée de 13,5 millions en 1958 à 30,7 millions d'habitants en 1984. En 2007, I'Institut National de la Statistique (INS) cité par EDS-RDC de 2007 l'estimait à 65,8 millions d'habitants, avec près de 8 millions dans la seule ville de Kinshasa, la capitale du pays. Quant au taux d'accroissement naturel de la population, il est estimé à 3,5\% pour la période 20052010 (UNDP, 2009).

Si l'on s'en tient à l'hypothèse moyenne des projections des Nations Unies, qui est l'une des sources les plus utilisées, la population congolaise est estimée à 69781411 d'habitants en 2012 avec un taux d'accroissement annuel de la population de $2,7 \%$ entre 2009 et 2010 . Cette forte dynamique démographique si elle restait constante, entraînerait un doublement de la population congolaise tous les 26 ans.

\subsubsection{Le système éducatif congolails : politique et stratégies éducatives}

\section{a) Structuration actuelle du système éducatif}

En RDC, le système éducatif est administré par trois ministères : le Ministère de l'Enseignement Primaire, Secondaire et Professionnel (EPSP), le Ministère de l'Enseignement Supérieur et Universitaire (ESU) et le Ministère des Affaires Sociales (MAS). Toutefois, d'autres ministères tels que les ministères de la Recherche scientifique, de l'emploi et de prévoyance sociale, de la santé et de la jeunesse et des sports sont également impliqués dans le système éducatif à travers certaines activités.

Au niveau central chaque ministère est géré par un ministre nommé par le Président de la République et au niveau provincial, par un ministre provincial nommé par le Gouverneur. L'ensemble des services administratifs et pédagogiques est sous la direction d'un Secrétariat Général (SG) qui exécute la politique du Gouvernement en matière d'éducation (Banque Mondiale, 2005).

Le ministère de l'EPSP est divisé en 30 provinces éducationnelles. Le ministère est représenté dans les provinces par des divisions provinciales (PROVED) et sous-provinciales (Sous-PROVED). Au niveau provincial, le Ministre de l'EPSP est donc représenté par le chef de division provinciale qui est lui aussi représenté par les chefs de sous-division au niveau sous-provincial. L'EPSP comporte trois niveaux d'enseignement : pré-primaire $\left(\right.$ CITE $0^{2}$ ), primaire (CITE 1 ) et secondaire (CITE 2 et 3). Le pré-primaire, organisé en un cycle de trois ans, est facultatif. Il est géré en grande partie par le secteur privé et accueille les enfants âgés de 3 à 5 ans. Le niveau d'enseignement primaire dure 6 ans et concerne les enfants âgés de 6 à 11 ans. Le niveau d'enseignement secondaire comprend 4 cycles : (i) le cycle long (humanités) d'une durée de 6 ans qui donne accès aux études supérieures et universitaires, est subdivisé en deux sous-cycles, un premier cycle de 2 ans pour les enfants de 12-13 ans (CITE 2) et le second cycle concerne les enfants de 14-17 ans (CITE 3); (ii) le cycle Spécialisation Professionnelle (CSP) qui dure 1 ou 2 ans ; (iii) le cycle des Arts et Métiers d'une durée de 1 à 3 ans et (iv) le cycle Professionnel d'une durée de 4 à 5 ans (RDC, 2010).

\footnotetext{
2 Des détails peuvent être obtenus sur la correspondance des cycles en RDC avec la classification internationale type de l'éducation sur le site de l'UIS : http://www.uis.unesco.org/Education/ISCEDMappings/Pages/defaultFR.aspx?SPSLanguage=FR.
} 
L'enseignement supérieur comporte un premier cycle de trois ans (CITE 5) et un second de deux à trois ans selon les filières (CITE 6). Trois types d'enseignement supérieur sont offerts : l'enseignement supérieur universitaire, l'enseignement supérieur pédagogique et l'enseignement supérieur technique.

Le Ministère des Affaires Sociales (MAS) est chargé de l'éducation non-formelle, notamment le rattrapage scolaire, l'alphabétisation des jeunes et des adultes, l'apprentissage professionnel et l'éducation permanente des adultes.

D'autres acteurs, notamment les parents, interviennent également dans le système éducatif congolais. En effet, les parents constituent un des acteurs majeurs de l'administration du système scolaire congolais. Ils sont représentés par des comités de parents dans les écoles et les coordinations. Ces associations ont pour rôle d'inciter les parents à scolariser leurs enfants et à coopérer à la gestion des écoles.

Sur le plan organisationnel, on dénombre quatre catégories d'écoles en RDC : (i) Les écoles directement sous le contrôle de l'État; (ii) Les écoles des réseaux, généralement confessionnels, en convention avec l'État, dites conventionnées; (iii) les écoles privées agréées par l'État; et (iv) les écoles privées non agréées par l'État. Les deux premiers types d'écoles sont qualifiés d'écoles publiques et les autres d'écoles privées (RDC, 2010).

\section{b) Politique actuelle}

Une stratégie pour le développement du sous-secteur de l'enseignement primaire, secondaire et professionnel a été adoptée par le Gouvernement en mars 2010. Dans l'attente de la finalisation de la stratégie globale du secteur de l'éducation ${ }^{3}$, le MEPSP a élaboré un Plan intérimaire de l'Education (PIE) pour la période 20122014. Celui-ci a pour objectifs :

- l'accroissement de l'accès et de l'accessibilité à l'enseignement primaire ;

- l'amélioration de la qualité de l'enseignement et des apprentissages scolaires ;

- le renforcement de la gouvernance.

Pour atteindre ces objectifs, le PIE est structuré en dix (10) programmes dont :

trois (3) programmes visant l'accroissement et l'amélioration de l'offre et de la demande d'éducation (faciliter l'accès au préscolaire, alléger la charge financière des ménages par la prise en charge des frais scolaires par l'Etat, construire et réhabiliter les établissements scolaires) ;

- quatre (4) visent l'amélioration de la qualité des apprentissages et de la pertinence de l'enseignement (à travers, entre autres, la refondation de la formation initiale et continue des enseignants, la dotation des écoles en supports et matériels pédagogiques...) ;

- trois (3) programmes visent le renforcement des capacités et acteurs du système éducatif.

\subsection{Approche globale de l'analyse des EADE: les cinq dimensions de l'exclusion}

Sur la base de la définition des EADE (UNICEF et UNESCO Institute of statistics, 2010), la recherche se fait autour des cinq dimensions de l'exclusion qui comprend deux grands groupes d'enfants (graphique 1). Les enfants d'âge scolaire en dehors de l'école (dimensions 1, 2 et 3) et les enfants à l'école mais présentant un grand risque d'abandonner (dimensions 4 et 5). Ces différentes dimensions sont définies ainsi qu'il suit :

- Dimension 1 : les enfants d'âge scolaire pré-primaire qui ne sont pas scolarisés en pré primaire ou primaire;

- Dimension 2 : les enfants d'âge scolaire primaire qui ne sont pas scolarisés ni au primaire ni à un degré supérieur;

- Dimension 3 : les enfants d'âge scolaire du $1^{\text {er }}$ cycle du secondaire qui ne sont pas scolarisés ni au primaire, ni au secondaire, ni à un degré supérieur

- Dimension 4 : les enfants scolarisés au primaire mais qui risquent d'abandonner;

- Dimension 5 : les enfants scolarisés au $1^{\text {er }}$ cycle du secondaire et qui risquent d'abandonner.

\footnotetext{
${ }^{3}$ Les deux autres ministères intervenant dans le secteur de l'éducation, le Ministère de l'Enseignement Supérieur et Universitaire (MESU) et le Ministère des Affaires Sociales (MAS) poursuivent le processus de finalisation de leurs stratégies sous-sectorielles.
} 
Graphique 1 : Illustration des cinq dimensions de l'exclusion (5DE)

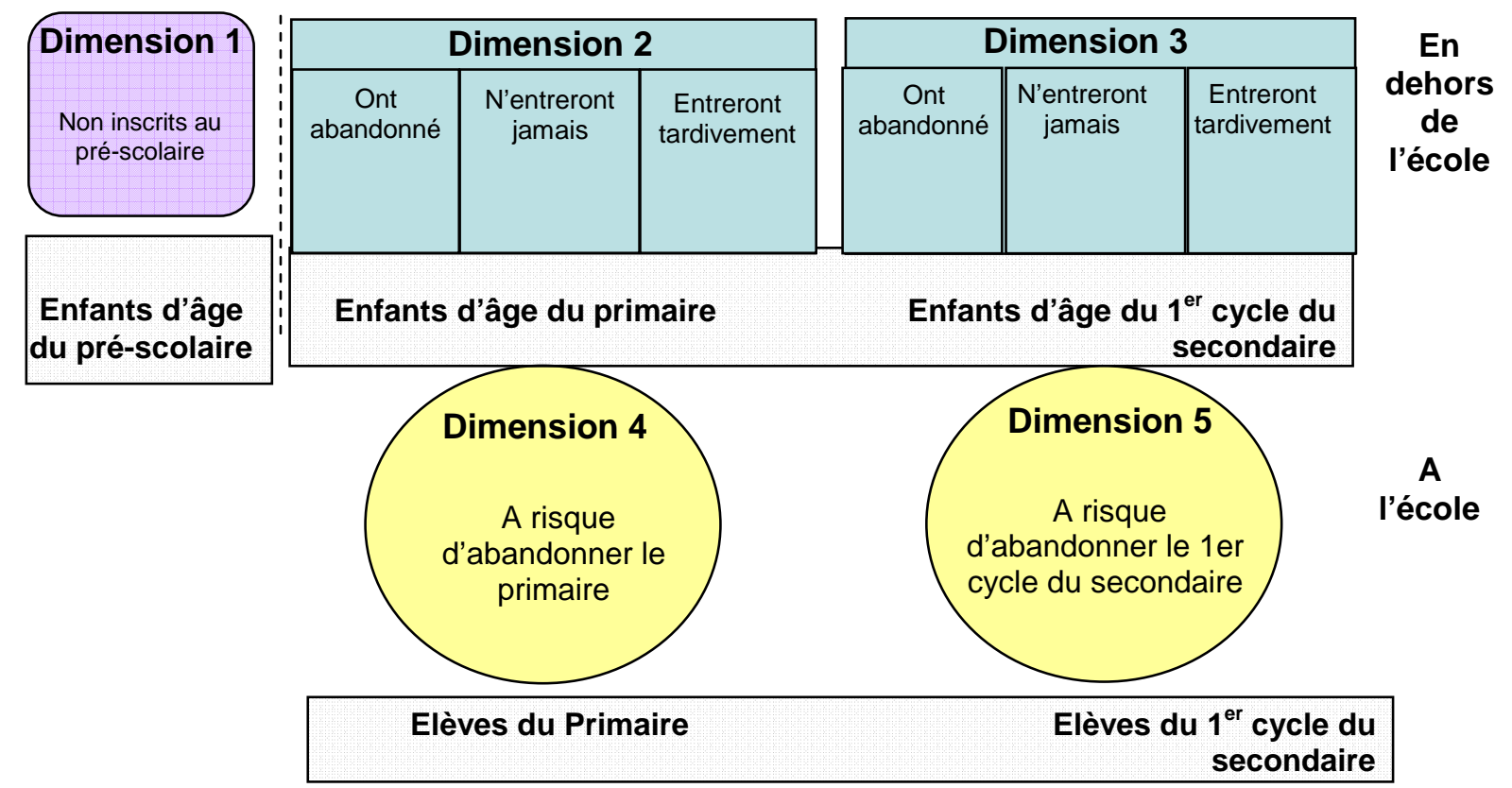

Source : UNICEF et UNESCO Institute of statistics (2011)

L'étude sur les enfants et adolescents a voulu aller au-delà des tranches conventionnelles du Cadre conceptuel et méthodologique (CMF), comme convenu à l'atelier national méthodologique. Étant donné que la tranche d'âge officiel pour le pré-primaire est de 3-5 ans en RDC, tout en sortant les résultats pour les 5 ans (comme dans le CMF), l'étude EADE-RDC donne également les chiffres de l'exclusion pour les 3-5 ans. Par ailleurs, l'EADE-RDC donne en plus des résultats de la dimension 3 ( $\left({ }^{\mathrm{er}}\right.$ cycle du secondaire), des résultats de l'exclusion scolaire pour les enfants d'âge du second cycle du secondaire (14-17 ans).

II convient de faire un commentaire sur les groupes d'âges à considérés. En effet, comme le mentionne le Cadre conceptuel et méthodologique (CMF), l'âge des enfants à considérer dépend du moment de la collecte des données. Lorsque le moment de la collecte dépasse largement six (6) mois par rapport au début de la rentrée scolaire, les enfants et adolescents qui sont enquêtés au moment de l'enquête étaient en réalité jeunes d'un an en moyenne au moment de la rentrée scolaire. II peut être alors nécessaire dans ce cas d'ajouter un an de plus à l'âge des enfants pour ne pas surestimer l'ampleur de l'exclusion scolaire. Dans le cadre de l'EADE-RDC, l'enquête a eu lieu du 8 mars au 31 mai 2012, alors que la rentrée scolaire a eu lieu en septembre 2011, ce qui fait une durée après la rentrée allant de 6 mois (pour ceux enquêtés en mars) à 8 mois (pour ceux enquêtés en mai). Rigoureusement, en suivant la directive du CMF, pour ceux enquêtés en avril et mai, il aurait fallu considérer les tranches d'âge scolaires, majorées d'une année pour apprécier leur niveau d'exclusion de l'école (au lieu de 5-17 ans, il aurait donc fallu considérer les 6-18 ans). Toutefois, en procédant ainsi, nous obtenons une proportion d'EADE de 24,5\% (pour les 6-18 ans) ce qui comparé au MICS 2010 (32,5\% d'EADE), donnerait un écart de 8 points sur une période de deux ans (2010-2012). Un tel écart semble difficilement soutenable compte tenu des tendances entre 2007 et 2010 (38,5\% à l'EADE 2007). Par conséquent, dans toute la suite, les tranches d'âges sont maintenues telles que dans le CMF. 


\subsection{Méthodologie de l'enquête nationale sur la situation des EADE}

\subsubsection{Spécificités et apports de l'enquête EADE}

Tirant les enseignements de l'état des lieux, notamment l'exploitation des enquêtes auprès des ménages et des autres rapports et études, l'étude EADE a réalisé des collectes de données spécifiques tout en prenant en compte des questions très peu ou pas du tout abordées dans les sources de données existantes. Dans le volet quantitatif, trois collectes ont été réalisées : une enquête auprès des ménages, un recensement des enfants de la rue et un recensement des structures d'accueil des enfants et adolescents.

\section{a) Enquête auprès des ménages}

Le questionnaire "ménage " a permis d'enregistrer tous les membres du ménage avec certaines de leurs caractéristiques démographiques et socio-économiques : lien de parenté avec le chef de ménage, sexe, âge, situation de résidence, niveau d'instruction, activité économique, etc. Le but premier du questionnaire ménage était de fournir les informations permettant de déterminer les populations de référence pour le calcul des taux dans le domaine éducatif (scolarisation, fréquentation scolaire, etc.), et d'identifier les enfants de 3-5 ans et de 6-17 ans éligibles pour les interviews individuelles.

Le questionnaire ménage contenait également des informations relatives aux conditions de vie du ménage, notamment les caractéristiques de l'habitat, la possession de biens durables et non durables, la production agricole, le revenu du ménage, les dépenses d'éducation et la situation des enfants du ménage vivant ailleurs.

Les questionnaires "enfant de 3-5 ans » et "enfant de 6-17 ans » ont été utilisés pour enregistrer les informations concernant chaque catégorie d'enfants. Ils comprenaient, chacun, les trois sections suivantes :

- Caractéristiques sociodémographiques de l'enfant ;

- Éducation ;

- Travail des enfants.

Le questionnaire des 3-5 ans était adressé à un des parents de l'enfant ou une personne de référence, tandis que celui des 6-17 ans était administré à l'enfant ou l'adolescent lui-même.

\section{b) Recensement des enfants dans les structures d'accueil}

Une «fiche structure " a été élaborée en vue du recensement des institutions d'accueil des enfants et adolescents. Elle comprenait en plus des informations sur l'identification de la structure, la liste exhaustive des personnes qui résident dans la structure avec leurs caractéristiques socio-démographiques (sexe, âge et lieu de naissance), l'information sur leur statut scolaire et sur la survie des parents ainsi que l'existence ou pas de contacts avec les parents biologiques (s'ils sont en vie) ou d'autres parents.

\section{c) Recensement des enfants de la rue}

Pour les enfants ou adolescents vivant dans la rue, une «fiche enfant de la rue " a été élaborée. Elle comprenait les informations sur la localisation géographique du site, les caractéristiques sociodémographiques des enfants recensés sur le site (sexe, âge et lieu de naissance), sur leur statut scolaire et sur la survie des parents ainsi que l'existence ou pas de contacts avec les parents biologiques (s'ils sont en vie) ou d'autres parents, et s'il leur arrive de fréquenter une structure d'accueil tout en étant dans la rue.

\subsubsection{Définition de quelques principaux concepts}

\section{a) Travail des enfants}

La définition du travail des enfants est généralement complexe. Cette complexité est en rapport avec les fondements mêmes du travail des enfants qui sont de natures diverses : fondements culturels (Erny, 1972 ; Schlemmer, 1996, Salazar, 1998) mais aussi fondements économiques en rapport avec le mode de production (Kamuzora, 1984 ; Meillassoux, 1992) ou en rapport avec la pauvreté (Brisset, 2000 ; Unicef, 1997). Mais entre le travail socialisateur et le travail économique de l'enfant, la frontière n'est pas toujours distincte, comme entre le travail économique et les pires formes du travail des enfants. 
Deux définitions du travail seront considérées ici pour mettre en évidence comment le choix d'une définition donnée influe sur l'ampleur de la participation des EADE au travail :

- la première définition est celle d'une acception générale du travail des enfants, au sens d'« activité économique ", c'est-à-dire l'ensemble des activités productives menées par les enfants que celles-ci soient marchandes ou non (Diallo, 2006);

- la deuxième définition est celle de UCW (Understanding Children's Work) utilisée dans le cadre de la méthodologie de l'Initiative mondiale sur les EADE (ISU-UNESCO et UNICEF, 2010) qui définit le travail des enfants en termes de nombre d'heures de travail économique effectué par l'enfant au cours de la période de référence. Cette définition est une opérationnalisation de la définition adoptée lors de la $18^{\text {ème }}$ Conférence internationale des statisticiens du travail en 2008 (ILO, 2009).

\section{b) Ménage et Chef de ménage}

Un ménage (ordinaire) est un ensemble de personnes apparentées ou non reconnaissant l'autorité d'un même individu appelé "Chef de ménage". Elles habitent le plus souvent sous un même toit, dans la même cour ou dans la même concession. Elles prennent le plus souvent leurs repas ensemble et partagent les dépenses courantes du ménage.

Le chef de ménage est la personne responsable de l'entretien ou de la prise en charge du ménage.

\section{c) Enfant du ménage et enfant vivant ailleurs}

L'importance des réseaux de solidarités familiales en Afrique implique généralement que l'investissement des parents et des familles dans l'éducation va au-delà des propres enfants biologiques du couple et s'étend bien souvent à d'autres enfants et adolescents de la parentèle. Aussi, se limiter aux seuls enfants résidant dans le ménage que ce soit en termes de niveau de scolarisation ou en termes d'investissement économique peut biaiser l'estimation de l'ampleur de la contribution des ménages à la scolarisation des enfants.

Par enfant du ménage nous entendons deux catégories d'enfants : (i) les enfants résidant dans le ménage au moment de l'enquête, incluant les propres enfants biologiques du chef de ménage et de son (ou ses) conjoint(s) et les autres enfants (apparentés ou non) ; (ii) les enfants biologiques du chef de ménage et de son (ou ses) conjoint(s) vivant ailleurs.

Par enfant vivant ailleurs, nous entendons deux groupes d'enfants: (i) les enfants biologiques du chef de ménage et de son (ou ses) conjoint(s) vivant ailleurs (qui sont aussi des enfants du ménage) et (ii) les autres enfants autres que les enfants biologiques du chef de ménage et de son (ou ses) conjoint(s) vivant ailleurs et dont le ménage contribue au financement de la scolarisation.

\section{d) Pauvreté}

La pauvreté est une "privation prononcée de bien-être " ("pronounced deprivation in well-being") (World Bank, 2001, p. 15). La privation pouvant relever de divers aspects de la vie humaine (revenu, alimentation, logement, santé, éducation, etc.), la pauvreté est un phénomène résolument multidimensionnel, recouvrant aussi bien la privation matérielle ou pauvreté monétaire (income poverty) que la pauvreté non monétaire, notamment les besoins essentiels non satisfaits (approche B.E.N.S) et la question des capacités (“capabilities") introduite par Amartya Sen (1992).

Quelle que soit leur diversité, toutes les définitions de la pauvreté peuvent être regroupées (Hagenaars et De Vos, 1988, p. 212), en trois catégories : i) la pauvreté absolue (la pauvreté c'est avoir en deçà d'un minimum absolu défini ; ii) la pauvreté relative (la pauvreté c'est avoir moins que les autres dans la société), et iii) la pauvreté subjective (la pauvreté c'est estimer qu'on n'a pas assez pour survivre).

L'un des objectifs de la recherche EADE est d'examiner l'effet du revenu sur les risques d'exclusion des enfants et adolescents du système éducatif. Par conséquent, c'est l'effet de la pauvreté monétaire que nous cherchons à capter. Compte tenu de la difficulté de collecter des informations sur les dépenses de consommation dans une enquête comme celle-là ayant plusieurs autres objectifs (pour des raisons de temps et de dispositifs) ou sur le revenu exact du ménage (réticence à donner son revenu exact), l'enquête EADE a demandé aux ménages de se situer par rapport à des classes de revenu mensuel déjà prédéfinies («moins de 50 USD », « 50 à 100 USD », 101 à 200 USD, « 201 à 500 USD », « 501 à 1000 USD » et «Plus de 1000 USD »). 


\subsubsection{Eléments sur l'échantillonnage}

\section{a) L'échantillonnage des données quantitatives}

- Enquête sur les enfants et adolescents vivant dans les ménages :

La procédure d'échantillonnage suivie dans l'enquête EADE-RDC 2012, suit celle adoptée par les autres enquêtes nationales passées, notamment l'EDS 2007 et le MICS 2010.

\section{Plan de sondage}

Le processus d'échantillonnage mis en œuvre est présenté suivant les éléments suivants : la nature de l'enquête, le champ d'investigation, les niveaux de stratification, les unités de sondage, la base de sondage, le mode de tirage, le calcul de la taille de l'échantillon. Ce plan de sondage concerne l'échantillon des enfants vivant dans les ménages.

\section{Nature d'enquête et champ}

C'est un sondage par grappe stratifiée et à plusieurs degrés. Elle couvre toutes les 11 provinces du pays. Tous les ménages ordinaires résidant sur l'ensemble du territoire national sont concernés à l'exception des ménages collectifs et ceux du corps diplomatique.

\section{La stratification}

Chacune des 10 provinces a été découpée en trois strates qui sont le milieu rural, les villes statutaires et les cités. La ville-province de Kinshasa a été découpée en 4 strates correspondant aux 4 anciens districts. On aboutit ainsi à un total de 34 strates. Le tirage des unités de sondage s'est fait de façon indépendante dans chaque strate.

\section{Les unités de sondages}

Les unités de sondages sont définies comme suit :

a) Dans la strate des villes statutaires:

- Au 1er degré (unité primaire) = le quartier

- Au 2è degré (unité secondaire) = le ménage

b) Dans la strate des cités :

- Au 1er degré (unité primaire) = la cité

- Au 2è degré (unité secondaire) = le quartier

- Au 3è degré (unité tertiaire) = le ménage

c) Dans la strate rurale :

- $A u 1^{\text {er }}$ degré (unité primaire) = le secteur/la chefferie

- Au 2è degré (unité secondaire) = le village

- Au 3è degré (unité tertiaire) = le ménage

\section{La base de sondage}

Les recensements sont en général utilisés en Afrique comme base de sondage pour atteindre les ménages et les individus dans les ZD (zone de dénombrement) ou SE (Secteur d'énumération). En RDC, les unités les plus fines dont on dispose d'une base de sondage sont les chefferies/secteurs en milieu rural, et les quartiers/cités en milieu urbain. Ces unités ont constitué notre base de sondage. Cette liste (base de sondage) a été mise à jour en 2010 pour les besoins du MICS 2010.

\section{Mode de tirage}

Les unités (quartiers ou cités ou secteurs/chefferies, segments), sont tirées avec des probabilités proportionnelles à la taille (effectifs de population) tandis que les ménages ont été sélectionnés par un tirage systématique à probabilités égales. Quand un village ou un quartier est tiré, on procède à un dénombrement des ménages. Si le nombre de ménages compté est inférieur ou égal à 500 , on tire les 30 ménages. Par contre, si le nombre de ménages comptés est supérieur à 500 on procède à la segmentation qui est un découpage en des segments d'environ 300 ménages. On tire ensuite un segment et dans ce segment on tire les 30 ménages. Pour plus de détails sur le mode de tirage, voir l'annexe 5 sur l'échantillonnage. 


\section{Taille d'échantillon et précision}

La taille de l'échantillon a été estimée de telle sorte qu'elle puisse permettre d'assurer une représentativité de l'indicateur clef (proportion des enfants et adolescents en dehors de l'école) au niveau de la catégorie d'âge ayant la plus faible importance en termes d'effectif, donc les 12-13 ans. Aussi en assurant la représentativité au niveau de ce groupe d'âge, la représentativité est alors assurée au niveau des autres groupes d'âges plus importants en effectif à savoir : les 3-5 ans ; les 6-11 ans ; les 14-17 ans. Cette représentativité est aussi assuré au niveau de l'urbain et du rural de chaque province pour les 6-11 ans et les 14-17 ans. Pour les groupes d'âges 3-5 ans et 12-13 ans, la représentativité n'est assurée qu'au niveau province. L'urbain/rural n'est assuré qu'au niveau national. La distinction entre villes statutaires et cités au niveau des indicateurs n'est assurée avec une bonne précision qu'au niveau national. La formule utilisée pour le calcul de la taille de l'échantillon est la suivante :

$$
n=\frac{z^{2} r(1-r) * \text { deff } *(1+t)}{\mathcal{E}^{2 *}\left(p^{*} n_{h}\right)}
$$

Avec:

$n=$ Taille de l'échantillon (en termes de nombres de ménages);

$z^{2}=$ Constante issue de la loi normale pour un seuil de confiance donnée ;

$r=$ proportion de l'estimateur clef recherché ;

deff = effet du plan de sondage (ou effet de grappe) ;

$t=$ taux de non réponse ;

$\mathcal{E}^{\mathbf{2}}=$ Marge d'erreur ;

$p=$ est la proportion du sous-groupe dans la population totale ;

$\mathrm{nh}=$ est la taille moyenne du ménage ;

L'estimation de la taille de l'échantillon a été faite pour chacune des 34 strates à partir des informations issues du MICS 2010 de la RDC. Les valeurs standards retenues pour l'estimation de la taille sont : une précision de 0,05 soit $z^{2}=3,84$; un effet de grappe de 1,5 ; un taux de non réponse de $10 \%$ et une erreur $\varepsilon=0,05$. Les valeurs de la proportion des EADE dans le groupe d'âge 12-13 ans pour chacune des 34 strates sont données en annexe. On obtient alors en appliquant la formule précédente dans chaque strate, à un échantillon total de 13611 ménages et une estimation attendue 30869 enfants de 3-17 ans. Le tableau A1 en annexe sur l'échantillonnage donne la répartition de l'échantillon par strate.

- Recensement des enfants vivant dans les institutions et dans la rue :

L'un des apports de l'enquête EADE-RDC 2012 est la collecte des données sur les enfants en situation de rupture familiale, c'est-à-dire les enfants dans les institutions d'accueil et les enfants vivant dans la rue.

Pour les enfants dans les structures d'accueil, nous avons dans un premier temps récupéré la liste des structures d'accueil au niveau central auprès du Ministère de l'Action Sociale et de la Solidarité. Ces listes ont été ensuite complétées sur la base des entretiens réalisés au niveau local dans toutes les onze provinces, avec les organisations travaillant dans le domaine de l'enfance. Les structures ont été toutes visitées à l'aide d'une "Fiche structure » conçue à cet effet et collectant un certain nombre d'informations sur la structure et établissant la liste exhaustive de toutes les personnes accueillies dans la structure.

Au total, 232 structures ont été recensées dans les 11 provinces avec un effectif total de 13499 personnes accueillies. Parmi elles, les enfants et adolescents de 5-17 ans sont au nombre de 11301 individus, soit 83,9 $\%$.

- Recensement des enfants vivant dans la rue :

En ce qui concerne les enfants vivant dans la rue, la démarche a consisté dans un premier temps à s'entretenir avec les organisations de la société civile intervenant dans le domaine afin d'avoir quelques premiers éléments de connaissance générale sur le phénomène et la démarche la mieux indiquée pour dénombrer ces enfants et adolescents vivant dans la rue. Cette collecte a porté sur l'ensemble des grandes agglomérations des onze provinces du pays.

Les informations obtenues au niveau central sur le phénomène et les organisations de la société civile intervenant au niveau local auprès des enfants et adolescents vivant dans la rue, ont été ensuite actualisées sur le terrain avec les acteurs locaux. Ensuite, avec l'aide d'informateurs, les espaces de vie de ces enfants et adolescents ont été identifiés et une "fiche enfant vivant dans la rue " a été utilisée pour recueillir un ensemble d'informations sur les personnes dénombrées. L'identification et le dénombrement ont été faits le soir afin de ne retenir que les enfants qui dorment dans la rue. 
Les enfants et adolescents vivant dans la rue sont ceux qui y vivent et qui y dorment. Ils se composent exclusivement des enfants et adolescents n'ayant d'espace de vie que la rue. Ceux qui vivent dans la rue et qui n'y dorment pas ne sont donc pas concernés et par conséquent n'ont pas été pris en compte dans le recensement. Les enfants et adolescents vivant dans la rue sont une catégorie de population très spécifique qui vit par petit groupe et dans des espaces bien déterminés. Dans la journée, ces populations se dispersent dans la ville à la recherche de quoi survivre dans les espaces publics (services, abords de boutiques, banques, marchés, les coins de rue, axes routiers, restaurants, etc.). Elles se mêlent de ce fait donc à une autre catégorie de la population qui ne résident pas forcément dans la rue mais qui est contrainte de fréquenter aussi ces milieux pour des raisons de survie. Leur recensement c'est fait dans la nuit. Pour atteindre cette catégorie d'enfants et adolescents ayant élu domicile dans la rue, l'approche suivante a été adoptée par l'EADE-RDC de 2012 :

Répertoire des espaces de vie et identification des informateurs clés

Les agents de terrain dans les centres urbains, avec l'aide de la population, des autorités administratives, coutumières et religieuses ainsi que des leaders d'opinion, ont répertorié les espaces où dorment chaque groupe d'enfants et adolescents. Chaque groupe a son "protecteur" qui est un adulte et sur qui les enquêteurs EADE-RDC se sont appuyés pour pouvoir approcher les membres du groupe.

\section{Moments des interviews}

Dans la journée, il a été quasiment impossible de rencontrer les enfants et adolescents sur le site où ils passent la nuit. II a donc fallu avec l'informateur clé (le protecteur du groupe) faire des arrangements pour les recenser la nuit.

\section{Durée de la collecte}

Le recensement des enfants vivant dans la rue a duré toute la période de l'enquête EADE-RDC de 2012, soit un mois et demi, ceci dans le souci de permettre aux agents de collecte d'effectuer plusieurs visites de rappel en vue de trouver les enfants et pouvoir les recenser.

Au total, 212 espaces de vie ont été recensés, regroupant un effectif de 5915 personnes, dont 4365 (73,8\%) sont des enfants et adolescents de 5-17 ans.

\section{Situation d'autres approches sur les enfants et adolescents vivant dans la rue}

Le Réseau des Éducateurs des Enfants et Jeunes de la Rue (REEJER) a conduit en 2006 un recensement des enfants de la rue dans la ville province de Kinshasa. Cette opération, initialement prévue, pour être conduite la nuit a finalement été menée dans la journée pour des raisons de sécurité. L'opération qui s'est déroulée en 2 jours (les 6 et 7 octobre $2006^{4}$ ) a mobilisé 720 agents recenseurs.

\section{Comparaison des approches}

Pour la ville province de Kinshasa, l'EADE-RDC de 2012 a dénombré au total 2446 personnes dont 1578 de 0-17 ans qui vivent effectivement dans la rue. Le REEJER lors du recensement de 2006, a dénombré pour la ville province de Kinshasa, un effectif de 18098 (14 906 en milieu ouvert et 3192 en milieu fermé). Le milieu ouvert (ou site ouvert) s'entend "la rue au sens large du terme, c'est-à-dire comprenant places publiques, marchés, terrains vagues, bâtiments abandonnés etc. devenue la demeure habituelle de l'enfant sans protection ni encadrement". Le milieu fermé a été défini par "centre d'hébergement ou de transit des enfants de la rue" (REEJER, 2006).

Les différences observées entre les chiffres de l'EADE-RDC de 2012 et ceux du recensement du REEJER de 2006 proviennent sans nul doute des approches méthodologiques suivies et probablement de l'évolution positive du contexte qui s'est caractérisée par une stabilité des institutions qui se serait accompagnée d'une plus grande attention accordée à la situation des enfants en difficulté. De plus, la collecte des données de l'étude REJEER a été menée dans la journée pour des raisons de sécurité. On peut donc bien comprendre qu'elle a pris en compte des enfants et adolescents qui passaient la journée dans la rue sans forcément y passer la nuit. En effet, certains enfants passent la journée à faire diverses activités et retournent le soir à la maison ou en institution. Ce qui explique l'écart avec les chiffres du recensement des enfants de la rue réalisé en 2006 par le REEJER.

\footnotetext{
4 II convient de noter, en passant qu'Octobre 2006 correspondait au second tour des élections présidentielles en RDC.
} 


\section{b) L'échantillonnage des données qualitatives}

La recherche qualitative vise à fournir des informations sur les profils des enfants et adolescents en situation d'exclusion du système scolaire, examiner les raisons de leur non scolarisation et identifier les obstacles et goulots d'étranglement qui entravent la mise en œuvre des politiques éducatives.

Quatre provinces ont été sélectionnées pour la réalisation des entretiens qualitatifs auprès de divers acteurs de l'éducation. Tenant compte de la diversité de ces acteurs, plusieurs guides d'entretiens ont été élaborés pour la réalisation des entretiens. Ce sont :

- Trois guides d'entretiens individuels :

1- Guide d'entretien avec les parents, le personnel du système éducatif et les leaders communautaires ;

2- Guide d'entretien avec les partenaires techniques et financiers

3- Guide d'entretien avec les représentants des institutions et ONG travaillant dans le domaine de l'enfance et de la jeunesse

- Deux guides d'entretiens de groupes

1- Guide d'entretien avec les groupes d'enfants et adolescents vivant dans les ménages

2- Guide d'entretien avec les groupes d'enfants et adolescents sans attache familiale

De plus, des canevas de guides d'entretiens ont été élaborés pour la réalisation des études de cas. Au total 156 entretiens individuels, 80 entretiens de groupes et 13 études de cas ont été réalisés sur des thématiques d'intérêt spécifique constatées lors des entretiens dans les quatre provinces échantillonnées ainsi qu'au Katanga, Bas-Congo et Bandundu. Les entretiens individuels ont concerné les différents acteurs du système éducatif sans distinction de sexe (tableau 1). En revanche, pour les entretiens de groupes, dans chaque groupe cible, un nombre identique de groupes de garçons et de filles ont été interviewés. En plus du genre, les discussions de groupes ont aussi tenu compte de la diversité selon l'âge (enfants, adolescents), le milieu de résidence (urbain/rural), et le statut familial (membre d'un ménage/sans attache familiale).

Tableau 1 : Liste des entretiens qualitatifs réalisés

\begin{tabular}{|c|c|c|c|c|c|}
\hline \multirow{2}{*}{$\begin{array}{l}\text { Niveau de } \\
\text { collecte }\end{array}$} & \multirow{2}{*}{ Informateurs } & \multirow{2}{*}{$\begin{array}{l}\text { Entretiens } \\
\text { individuels }\end{array}$} & \multicolumn{2}{|c|}{ Entretiens ind / sexe } & \multirow{2}{*}{$\begin{array}{l}\text { Entretiens de } \\
\text { groupe }\end{array}$} \\
\hline & & & Hommes & Femmes & \\
\hline National & $\begin{array}{l}\text { Décideurs politiques, ministères, inspections } \\
\text { et directions de l'EPSP, PTF, coordination } \\
\text { nationale des écoles conventionnées }\end{array}$ & 20 & 17 & 3 & NC \\
\hline \multirow{17}{*}{$\begin{array}{l}\text { Province } \\
\text { (4/11) } \\
\text { 1-Kinshasa } \\
\text { 2-Equateur } \\
\text { (Ouest) } \\
\text { 3-Nord Kivu } \\
\text { (Est) } \\
\text { 4-Kasaï } \\
\text { oriental } \\
\text { (Centre sud) }\end{array}$} & Gouvernorat & 4 & 4 & 0 & \\
\hline & PROVED & 4 & 4 & 0 & \\
\hline & Sous-PROVED & 16 & 16 & 0 & \\
\hline & Inspection principale provinciale & 8 & 5 & 3 & \\
\hline & Institutions de prise en charge des enfants & 8 & 5 & 3 & \\
\hline & Directeurs d'écoles & 8 & 7 & 1 & \\
\hline & Enseignants & 16 & 13 & 3 & \\
\hline & Encadreurs & 8 & 1 & 7 & \\
\hline & Comité de parents d'élèves & 16 & 14 & 2 & \\
\hline & COGES & 16 & 16 & 0 & \\
\hline & Parents d'enfants & 24 & 20 & 4 & \\
\hline & Leaders Religieux & 4 & 4 & 0 & \\
\hline & Chef de quartier ou chef de village & 4 & 4 & 0 & \\
\hline & Ado. vivant dans ménages & & & & $\begin{array}{c}24 \text { (12 Garçons } \\
\& 12 \text { Filles) }\end{array}$ \\
\hline & Ado. en rupture familiale & & & & $\begin{array}{l}16 \text { (8 Garçons } \\
\& 8 \text { Filles) }\end{array}$ \\
\hline & Enfants vivant dans ménages & & & & $\begin{array}{c}24 \text { (12 Garçons } \\
\& 12 \text { Filles) }\end{array}$ \\
\hline & Enfants en rupture familiale & & & & $\begin{array}{l}16 \text { (8 Garçons } \\
\& 8 \text { Filles) }\end{array}$ \\
\hline $\begin{array}{l}\text { Sous total } \\
\text { province }\end{array}$ & & 136 & 113 & 23 & $\begin{array}{c}80 \text { (40 Garçons } \\
\text { \& 40 Filles) }\end{array}$ \\
\hline Total & & 156 & 130 & 26 & 80 \\
\hline
\end{tabular}

Source : Données de l'enquête qualitative, EADE-RDC 2012 
Au niveau national, 20 entretiens individuels ont été effectués auprès de décideurs politiques, des ministères, des partenaires techniques et financiers, coordination nationale des écoles conventionnées, inspections et directions du ministère de l'enseignement. Au niveau des provinces, 34 entretiens individuels et 20 focus groupes ont été réalisés dans chacune des quatre provinces. Le tableau ci-dessous présente la répartition des entretiens selon le profil des acteurs. Sur les 156 entretiens individuels réalisés, 26 femmes ont été interviewées contre 130 hommes, soit $16,7 \%$ de l'échantillon. Cette faible participation des femmes s'explique en partie par leur faible représentation dans les structures et institutions rencontrées.

\subsubsection{Méthodes d'analyse des données}

Pour l'exploitation des données de l'enquête EADE-RDC 2012, nous avons recours à plusieurs méthodes d'analyse.

\section{a) Analyse des données quantitatives}

En termes de méthodes quantitatives d'analyse, il a été fait recours à plusieurs méthodes. Plusieurs analyses descriptives, notamment des analyses bivariées ont été réalisées pour examiner la relation entre différentes variables et le fait d'être en dehors de l'école. Allant au-delà de ces analyses bivariées pour mieux apprécier l'effet net des différentes variables, des méthodes d'analyse multivariées explicatives ont été utilisées, notamment la régression logistique, compte tenu de la nature dichotomique de la variable dépendante, à savoir que l'enfant ou l'adolescent est en dehors de l'école ou pas au moment de l'enquête (voir annexe 3 sur la régression logistique pour plus de détails sur la méthode) $)^{5}$.

\section{b) Analyse des données qualitatives}

Les entretiens individuels et de groupe réalisés ont été enregistrés, traduits pour ceux réalisés en langues locales, transcrits, puis saisis sous forme de texte dans Microsoft Word par les agents de collecte. Ensuite, une dizaine d'agents de codification ont procédé à la codification des données. Les textes des entretiens qualitatifs ont été codifiés à l'aide du logiciel de traitement des données qualitatives NVivo. Les données ont été ensuite analysées à l'aide de la méthode d'analyse de contenu.

\subsubsection{Extrapolation des données}

Pour la réalisation de l'enquête EADE en RDC, un plan de sondage a été élaboré. Ce plan se fonde sur une base de sondage de I'INS mise à jour en vue de la réalisation de certaines enquêtes nationales comme l'Enquête 1-2-3. Cette base fournit les populations des différentes entités géographiques (jusqu'au niveau village et secteur) de la RDC. C'est cette base de sondage qui a été utilisée pour le tirage de l'échantillon. A partir des probabilités de tirage des différentes unités de sondage (secteur ou village), les coefficients de pondération (inverses des probabilités de tirage) ont été calculés. L'objectif de calculer les coefficients de pondération est d'extrapoler les résultats observés au niveau de l'échantillon à l'échelle de l'ensemble de la population, ce qui permet d'avoir également les chiffres absolus estimés dans l'ensemble de la RDC. D'autres paramètres ont été pris en compte dans le calcul de ces coefficients de pondération, à savoir les données de projections des Nations Unies pour la RDC en 2012 (pour caler, comme dans l'état des lieux, le chiffre global de la population sur la base de l'hypothèse moyenne des projections des Nations Unies) et les tendances observées dans la distribution de la population par province entre les enquêtes EDS-RDC 2007 et MICS-RDC 2010.

\subsubsection{Evaluation de la qualité des données}

L'évaluation des données, notamment de la structure par âge et sexe de la population enquêtée, révèle les mêmes erreurs de déclaration dans les âges qu'on rencontre dans les enquêtes auprès des ménages en Afrique, et se traduisant par le phénomène de l'attraction de certains âges (notamment les âges ronds se terminant par 0 et 5). Par ailleurs, l'examen de la pyramide par année d'âge a révélé quelques pics aux âges 18

\footnotetext{
5 Pour plus de détails sur l'ensemble de la méthodologie EADE, se référer au site web de l'étude EADE-RDC: http://eade.sulga.net/. Un processus de transfert vers le site de l'EPSP (http://www.eduquepsp.cd) est en cours.
} 
ans et 2 ans et donc des creux à 17 ans et 3 ans : il s'agit là très probablement de la tendance des enquêteurs sur le terrain à réduire le nombre de questionnaires individuels (3-5 ans et 6-17 ans), en "rajeunissant » certains enfants de 3 ans et "vieillissant 》 d'autres de 17 ans. Nous avons procédé au calcul d'un coefficient de correction que nous avons intégré dans les coefficients d'extrapolation en vue d'avoir un effectif de population scolarisable correspondant aux tendances observées.

La pyramide par groupes d'âges quinquennaux permet de corriger la distorsion dans la déclaration des âges. L'allure de la pyramide correspond bien à ce qu'on observe dans les pays à forte fécondité (base large de la pyramide) et un sommet qui s'effile au fur et à mesure qu'on avance en âge du fait de la forte mortalité (pour plus de détails sur cette évaluation de la structure par âge, se référer au document méthodologique sur le site web de l'étude EADE-RDC : http://eade.sulga.net/).

\subsection{Chiffres globaux de la population enquêtée}

\subsubsection{Enquête auprès des ménages}

Les 454 grappes sélectionnées pour l'échantillon de l'EADE-RDC de 2012 ont été couvertes. Au total, 13620 ménages ont été sélectionnés et, parmi eux, 13614 ménages ont été identifiés sur le terrain au moment de l'enquête. Parmi ces 13614 ménages identifiés, 13519 ont pu être enquêtés avec succès, soit un taux de réponse de 99,3\% (tableau 2). Avec une population estimée à 70255072 individus en 2012 selon les projections des Nations Unies, on estime à environ 13010199 le nombre de ménages en RDC pour une taille moyenne des ménages de 5,4 personnes. L'échantillon de l'EADE représente un peu plus du $1000^{\text {ème }}$ des ménages $(1,05 \%)$ et de la population $(1,04 \%$ ).

Tableau 2 : Effectif de ménages, nombre d'interviews individuelles et taux de réponse selon le milieu de résidence (Résultats non pondérés), RDC 2012

\begin{tabular}{|c|c|c|c|c|c|}
\hline \multirow{2}{*}{ Interviews } & \multicolumn{2}{|c|}{ Sexe } & \multicolumn{2}{|c|}{ Milieu } & \multirow[b]{2}{*}{ Ensemble } \\
\hline & Garçons & Filles & urbain & Rural & \\
\hline \multicolumn{6}{|l|}{ Interview des ménages } \\
\hline Ménages sélectionnés & na & na & 5324 & 8296 & 13620 \\
\hline Ménages identifiés & na & na & 5320 & 8294 & 13614 \\
\hline Ménages interviewés & na & na & 5275 & 8244 & 13519 \\
\hline Taux de couverture des ménages & na & na & 99,1 & 99,4 & 99,3 \\
\hline \multicolumn{6}{|l|}{ Interview des enfants de 3-5 ans } \\
\hline Effectifs d'enfants 3-5 ans éligibles & 4016 & 3856 & 3244 & 4628 & 7872 \\
\hline Effectifs d'enfants 3-5 ans éligibles interviewés & 3987 & 3828 & 3215 & 4600 & 7815 \\
\hline Taux de réponse des enfants de $3-5$ ans & 99,3 & 99,3 & 99,1 & 99,4 & 99,3 \\
\hline \multicolumn{6}{|l|}{ Interview des enfants de 6-17 ans } \\
\hline Effectifs d'enfants 6-17 ans éligibles & 10927 & 10534 & 9655 & 11806 & 21461 \\
\hline Effectifs d'enfants 6-17 ans éligibles interviewés & 10839 & 10448 & 9585 & 11702 & 21287 \\
\hline Taux de réponse des enfants de 6-17 ans & 99,2 & 99,2 & 99,3 & 99,1 & 99,2 \\
\hline
\end{tabular}

Dans les 13519 ménages enquêtés, 7826 enfants âgés de 3-5 ans ont été identifiés comme étant éligibles pour l'enquête individuelle, et pour 7817 d'entre eux, l'interview a été menée avec succès, soit un taux de réponse de 99,9\%. Pour les enfants de 6-17 ans, 21315 éligibles ont été identifiés et 21120 ont été interviewés (taux de réponse de 99,1\%). Au total, ce sont 28937 enfants de 3-17 ans qui ont été enquêtés sur 29141 identifiés (soit un taux de réponse global de 99,3\%). 
Tableau 3 : Taux de couverture (en \%) des enquêtes MICS et EDS de quelques pays

\begin{tabular}{|c|c|c|c|c|c|c|c|}
\hline \multirow[t]{2}{*}{ Pays/année } & \multicolumn{2}{|c|}{ Ménages MICS } & \multirow{2}{*}{$\begin{array}{c}\text { Taux de } \\
\text { couverture }\end{array}$} & \multirow[t]{2}{*}{ Pays/année } & \multicolumn{2}{|c|}{ Ménages EDS } & \multirow{2}{*}{$\begin{array}{c}\text { Taux de } \\
\text { couverture }\end{array}$} \\
\hline & Sélectionnés & Enquêtés & & & Sélectionnés & $\begin{array}{c}\text { Enquêté } \\
\mathrm{S}\end{array}$ & \\
\hline Cameroun 2006 & 9856 & 9848 & 99,9 & Cameroun 2011 & 14354 & 14214 & 99,0 \\
\hline RCA 2006 & 11940 & 11723 & 98,2 & Congo Brazzaville 2005 & 5926 & 5879 & 99,2 \\
\hline Nigeria 2007 & 28341 & 26735 & 94,3 & Rwanda 2010 & 12570 & 12540 & 99,8 \\
\hline Ghana 2006 & 6264 & 5939 & 94,8 & Tchad 2004 & 5399 & 5369 & 99,4 \\
\hline RCI 2006 & 7600 & 7600 & 100,0 & Sénégal 2010-11 & 8029 & 7902 & 98,4 \\
\hline
\end{tabular}

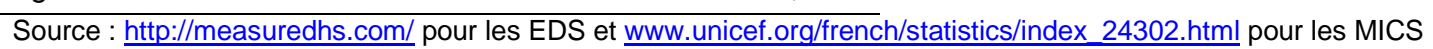

II apparaît ainsi que les différents taux de couverture sont très élevés et s'inscrivent dans ce qui est observés ailleurs dans d'autres enquêtes de même envergure en Afrique (tableau 3). Pour ce qui est de l'EADE-RDC 2012, il y a eu des campagnes de sensibilisation pour une participation des populations à l'enquête. Ces campagnes ont été menées par les radios et les télévisions publics et privés au niveau national et dans chacune des provinces. De plus, il y a eu la mobilisation communautaire par les responsables de quartier et village, ainsi que de l'administration publique. Ce sont autant d'arguments qui justifient ces taux de couverture élevés.

\subsubsection{Comparaison des échantillons et des taux couvertures avec les enquêtes EDS (2007) et MIICS (2010)}

Les trois dernières enquêtes d'envergure nationale, conduites en République Démocratique du Congo sur une méthodologie similaire, montrent que la taille des échantillons a progressivement augmenté d'une enquête à l'autre (tableau 4) de 27,8 \% et 18,3\%: 9000 ménages en 2007 (EDS-RDC) ; environ 11500 ménages en 2010 (MICS-RDC) et 13600 en 2012 (EADE-RDC). Du point de vue de la couverture, les taux montrent qu'elle a été bonne dans toutes les opérations avec des niveaux pratiquement identiques (99\%).

Tableau 4 : Effectif de ménages et taux de réponse selon le milieu de résidence EDS-RDC 2007, MICS-RDC 2010 et EADE-RDC 2012

\begin{tabular}{|c|c|c|c|c|}
\hline \multirow{2}{*}{ Enquête } & \multirow{2}{*}{ Interviews } & \multicolumn{2}{|c|}{ Milieu } & \multirow{2}{*}{ Ensemble } \\
\hline & & urbain & Rural & \\
\hline \multirow{4}{*}{ EADE } & Ménages sélectionnés & 5324 & 8296 & 13620 \\
\hline & Ménages identifiés & 5320 & 8294 & 13614 \\
\hline & Ménages interviewés & 5275 & 8244 & 13519 \\
\hline & Taux de couverture des ménages & 99,1 & 99,4 & 99,3 \\
\hline \multirow{4}{*}{ MICS } & Ménages sélectionnés & 4410 & 7080 & 11490 \\
\hline & Ménages identifiés & 4410 & 7079 & 11489 \\
\hline & Ménages interviewés & 4379 & 7014 & 11393 \\
\hline & Taux de couverture des ménages & 99,3 & 99,1 & 99,2 \\
\hline \multirow{4}{*}{ EDS } & Ménages sélectionnés & 3752 & 5250 & 9002 \\
\hline & Ménages identifiés & 3726 & 5219 & 8945 \\
\hline & Ménages interviewés & 3697 & 5189 & 8886 \\
\hline & Taux de couverture des ménages & 99,2 & 99,4 & 99,3 \\
\hline
\end{tabular}

\subsubsection{Enfants et adolescents en situation de rupture familiale}

Les personnes en situation de rupture familiale sont celles vivant dans la rue, mais aussi une partie de celles accueillies par les structures. Dans les structures on distingue les personnes qui vivent et qui dorment dans les structures. Celles là sont en rupture familiale. Mais il y a aussi celles qui sont prises en charge par la structure, mais qui retournent dormir chez elles le soir (tableau 5). Ces dernières ne sont donc pas en situation de rupture familiale. 
Tableau 5 : Récapitulatif des effectifs de personnes recensées dans la rue et dans les structures d'accueil par catégorie.

\begin{tabular}{|c|c|c|c|c|c|}
\hline \multirow{2}{*}{$\begin{array}{l}\text { Catégories } \\
\text { d'âges au } \\
\text { moment de } \\
\text { l'enquête }\end{array}$} & \multicolumn{3}{|c|}{ Personnes encadrées } & \multirow{2}{*}{$\begin{array}{l}\text { Personnes } \\
\text { de la rue }\end{array}$} & \multirow{2}{*}{$\begin{array}{l}\text { Personnes en } \\
\text { situation de rupture familiale } \\
\text { (de la rue et des structures) }\end{array}$} \\
\hline & $\begin{array}{l}\text { Dans une } \\
\text { Structure }\end{array}$ & En famille & Total & & \\
\hline $0-4$ & 556 & 214 & 770 & 63 & 619 \\
\hline $5-17$ & 5045 & 6256 & 11301 & 4365 & 9410 \\
\hline $18 \&+$ & 449 & 923 & 1372 & 1474 & 1923 \\
\hline ND & 14 & 42 & 56 & 13 & 27 \\
\hline Ensemble & 6064 & 7435 & 13499 & 5915 & 11979 \\
\hline
\end{tabular}

\section{a) Structures d'accueil}

L'enquête a dénombré 232 structures qui accueillent les enfants, et de façon plus générale, les personnes en situation difficile. C'est Kinshasa qui abrite la majorité des structures (38,4\%) suivi du Sud-Kivu (14,2\%). Le nombre important de structures à Kinshasa s'explique du fait que c'est la capitale où il y aurait plus d'opportunités. Quant au Sud-Kivu, c'est probablement dû à la situation de conflit armé dans cette partie du pays. On verra plus loin d'ailleurs que les enfants qui vivent dans ces structures au Sud-Kivu, viennent essentiellement du milieu rural, donc très probablement fuyant la guerre.

La taille moyenne d'une structure est de 102 personnes accueillies. Mais il y a une très forte disparité entre elles. On en trouve qui n'accueillent que seulement 4 personnes et il y en a de très grosses, surtout dans le Sud-Kivu.

Tableau 6 : Répartition (en \%) des personnes de 5-17 ans dans les structures d'accueil et vivant dans la rue, par province

\begin{tabular}{|c|c|c|}
\hline Province & $\begin{array}{l}\text { 5-17 ans accueillies dans } \\
\text { les structures d'accueil }\end{array}$ & 5-17 ans de la rue \\
\hline Kinshasa & 18,8 & 35,7 \\
\hline Bas-Congo & 1,5 & 3,0 \\
\hline Bandundu & 6,0 & 0,7 \\
\hline Equateur & 1,8 & 0,9 \\
\hline Province Orientale & 3,2 & 7,7 \\
\hline Nord-Kivu & 33,4 & 9,4 \\
\hline Maniema & 1,9 & 0,3 \\
\hline Sud-Kivu & 22,2 & 24,3 \\
\hline Katanga & 4,1 & 3,6 \\
\hline Kasaï Oriental & 4,7 & 8,2 \\
\hline Kasaï Occidental & 2,4 & 6,2 \\
\hline Total & 100,0 & 100,0 \\
\hline Effectifs & 11301 & 4365 \\
\hline
\end{tabular}

Source : Données du recensement auprès des structures d'accueil des enfants et du recensement des enfants de la rue, EADE-RDC 2012

Nous avons dénombré dans les structures 13499 personnes dont 11301 enfants et adolescents de 5-17 ans (83,7\%). C'est le Nord-Kivu qui vient en tête avec 33,4 \% des enfants accueillis, suivi du Sud-Kivu avec 22,2 $\%$ et de Kinshasa avec 18,8 \% (Tableau 6). Le nombre élevé des enfants accueillis dans les deux Kivu s'explique sans doute par la situation de conflit armée dans ces provinces.

Une forte proportion des enfants et adolescents dénombrés dans les structures d'accueil sont des orphelins $(46,5 \%)$. Près de $40 \%$ des enfants et adolescents dénombrés dans les structures d'accueil ont leurs deux parents biologiques en vie (tableau 7). Près d'un pensionnaire sur 5 est un orphelin complet. 
Tableau 7 : Statut familial des enfants 5-17 ans dans les structures et dans la rue

\begin{tabular}{lcc}
\hline Statut de l'enfant & $\begin{array}{c}5-17 \text { ans accueillis dans } \\
\text { les structures d'accueil }\end{array}$ & $\begin{array}{c}5-17 \text { ans dans } \\
\text { la rue }\end{array}$ \\
\hline Mère et père en vie & 39,6 & 35,4 \\
Orphelin complet & 17,2 & 13,9 \\
Orphelin de mère & 9,9 & 14,3 \\
Orphelin de père & 16,5 & 13,9 \\
Mère vivante situation du père inconnue & 4,1 & 4,6 \\
Père vivant situation mère inconnue & 0,9 & 1,8 \\
Père DCD Situation mère inconnue & 0,6 & 1,6 \\
Mère DCD situation père inconnue & 3,2 & 7,4 \\
Situation père et mère inconnue & 8,1 & 7,1 \\
\hline Total & 100,0 & 100,0 \\
Effectifs & 11301 & 4365
\end{tabular}

Source : Données du recensement auprès des structures d'accueil des enfants et du recensement des enfants de la rue, EADE-RDC 2012

\section{b) Enfants et adolescents vivant dans la rue}

Dans l'ensemble du pays, l'étude EADE-RDC a recensé au total 5915 personnes vivant dans la rue. Parmi ces personnes, $73,8 \%$ sont d'âge scolaire (5-17 ans). Les adultes de la rue représentent $24,9 \%$ soit le quart des effectifs. II y a cinq provinces où le phénomène des personnes vivant dans la rue est le plus important. II s'agit par ordre d'importance de Kinshasa, du Sud-Kivu, du Nord-Kivu, du Kasaï oriental, de la Province Orientale (tableau 8). Mais c'est à Kinshasa que le phénomène est le plus important avec plus de $41 \%$ des effectifs des personnes vivant dans la rue. Le Sud-Kivu qui arrive en deuxième position est de loin derrière. Le nombre important d'enfants vivant dans la rue dans les provinces du Kivu une fois encore est sans doute en lien avec les conflits dans cette province.

Même si le phénomène touche essentiellement les garçons, il est important de souligner la présence de filles vivant dans la rue. Elles représentent $18,2 \%$ de l'effectif des enfants en âge scolaire dans la rue. Quel que soit le sexe, les enfants vivant dans la rue ont dans leur grande majorité un âge compris entre 10 et 14 ans.

D'où viennent les enfants vivant dans la rue ? Près de $20 \%$ des enfants et adolescents vivant dans la rue sont nés en milieu rural. C'est dans les deux Kivu que les natifs du milieu rural sont proportionnellement les plus nombreux (tableau 8). Au Sud-Kivu, 50,5\% des enfants et adolescents vivant dans la rue proviennent du milieu rural. Cette proportion est de $34,9 \%$ dans le Nord Kivu. Une proportion non négligeable des enfants vivant dans la rue de la province de l'Equateur $(23,8 \%)$ provient aussi du milieu rural. Mais globalement, toutes les provinces confondues, ce sont les villes qui « produisent » les enfants vivant dans la rue et le milieu rural y contribue pour $20 \%$ environ. Les enfants vivant dans la rue à Kinshasa proviennent principalement de Kinshasa $(66,4 \%)$ et des capitales provinciales $(20,5 \%)$. 
Tableau 8 : Répartition (en \%) des enfants 5-17 ans vivant dans la rue selon le lieu de résidence et le lieu de naissance

\begin{tabular}{|c|c|c|c|c|c|c|c|c|}
\hline \multirow{2}{*}{$\begin{array}{l}\text { Province de } \\
\text { résidence }\end{array}$} & \multicolumn{7}{|c|}{ Lieu de naissance } & \multirow[b]{2}{*}{ Effectifs } \\
\hline & Kinshasa & $\begin{array}{l}\text { Capitale } \\
\text { provinciale }\end{array}$ & Autre ville & Cité & Rural & N.D & Total & \\
\hline Kinshasa & 65,6 & 21,0 & 6,2 & 2,4 & 4,8 & 0,0 & 100,0 & 1534 \\
\hline Bas-Congo & 18,6 & 17,8 & 39,5 & 13,2 & 10,9 & 0,0 & 100,0 & 129 \\
\hline Bandundu & 23,3 & 6,7 & 56,7 & 6,7 & 6,7 & 0,0 & 100,0 & 30 \\
\hline Equateur & - & 19,0 & 16,7 & 40,5 & 23,8 & 0,0 & 100,0 & 42 \\
\hline Province Orientale & 0,3 & 6,0 & 53,0 & 36,0 & 4,5 & 0,3 & 100,0 & 336 \\
\hline Nord-Kivu & 1,2 & 32,9 & 26,0 & 5,0 & 34,9 & 0,0 & 100,0 & 416 \\
\hline Maniema & - & 53,3 & 40,0 & 6,7 & - & 0,0 & 100,0 & 15 \\
\hline Sud-Kivu & 0,1 & 36,2 & 7,7 & 5,3 & 50,7 & 0,4 & 100,0 & 1073 \\
\hline Katanga & 1,3 & 31,4 & 42,1 & 11,3 & 13,8 & 0,0 & 100,0 & 159 \\
\hline Kasaï Oriental & 1,4 & 72,3 & 12,7 & 7,1 & 5,4 & 1,1 & 100,0 & 354 \\
\hline Kasaï Occidental & 3,3 & 35,4 & 36,9 & 12,2 & 12,2 & 0,0 & 100,0 & 271 \\
\hline Total RDC & 24,3 & 30,1 & 17,4 & 8,0 & 20,1 & 0,1 & 100,0 & 4359 \\
\hline
\end{tabular}

Source : Données du recensement des enfants de la rue, EADE-RDC 2012

Dans la conception populaire, la présence dans la rue est associée à l'absence de famille, de lien familial. La structure familiale étant le lieu de vie et d'évolution normale d'un enfant, la présence d'enfants vivant dans la rue amène à se questionner sur la famille d'origine de l'enfant, la survie de ses parents.

Parmi les personnes vivant dans la rue, on trouve $35,0 \%$ qui ont les deux parents en vie, $49,2 \%$ ont perdu un parent et $14,8 \%$ sont des orphelins de père et de mère (Tableau 9$)$. Pour les enfants en âge scolaire (5-17 ans), ces chiffres sont respectivement de $35,4 \% ; 50,5 \%$ et $13,9 \%$. Certains enfants ont déclaré ne pas connaitre la situation de leurs parents $(7,1 \%)$.

Tableau 9 : Comparaison de la répartition (en \%) selon la situation familiale des enfants vivant dans la rue, dans les structures d'accueil et dans les ménages

\begin{tabular}{lcccc}
\hline Catégorie d'enfants & $\begin{array}{l}\text { Non } \\
\text { orphelin }\end{array}$ & $\begin{array}{l}\text { Orphelin } \\
\text { d'un parent }\end{array}$ & $\begin{array}{c}\text { Orphelin } \\
\text { complet }\end{array}$ & Total \\
\hline Enfants vivant dans la rue & 35,4 & 50,7 & 13,9 & 100,0 \\
\hline Enfants dans les structures & 17,2 & 43,2 & 17,2 & 100,0 \\
\hline Enfants dans les ménages & 87,9 & 10,2 & 1,9 & 100,0 \\
\hline Source : Données de l'enquête auprès des ménages, du recensement auprès des structures d'accueil des enfants et du \\
recensement des enfants de la rue, EADE-RDC 2012
\end{tabular}

Le premier constat est qu'il y a proportionnellement plus d'orphelins parmi les enfants et adolescents vivant dans les structures d'accueil et dans la rue que les enfants vivant dans les ménages (Tableau 9). Le deuxième constat est que les enfants dans la rue ne sont pas nécessairement tous orphelins : plus d'un enfant ou adolescent dans la rue sur trois n'est pas orphelin. Dans de nombreux cas, ce n'est donc pas l'absence d'une famille qui est la cause du recours à la rue comme espace de vie mais bien d'autres facteurs. Des facteurs socio-économiques sont à la base de l'errance des enfants dans la rue. Parmi ceux-ci, on peut citer les divorces, les familles recomposées qui acceptent mal les enfants des unions précédentes, la perception même du bonheur par les enfants qui préfèrent quitter leurs parents qui sont trop pauvres, la fuite de responsabilité de certains parents, la pauvreté. Dans les structures d'accueil, il est rapporté que c'est souvent un parent qui vient confier l'enfant. Dans les discussions de groupe, les enfants ont avancé cinq types de raison à savoir :

- Le divorce ou la séparation des parents (rupture de l'union) ; « Mes parents ont divorcé et mon papa a pris une autre femme et ma maman a pris un autre homme. Je ne suis pas bien accepté dans cette nouvelle famille c'est pourquoi je suis parti. »

- Le décès du parent ;

- L'absence de prise en charge illustrée par ceci : « quand mon papa est décédé ma maman s'est mis dans le commerce et elle ne s'occupait plus de moi. Alors je suis parti. "

- La pauvreté des parents qui n'arrivaient plus à s'occuper des enfants. 
- La stigmatisation ou le bannissement: "Quand mon père/mère est décédé on m'a traité d'enfant sorcier. »

\subsection{Limites de l'étude}

Comme toute étude, l'enquête sur les EADE en RDC comporte des limites qu'il convient de mentionner.

\section{Au niveau de l'enquête quantitative:}

- II convient de souligner que comme dans toutes les autres enquêtes auprès des ménages, la question posée est la fréquentation scolaire ou non à un moment donné au cours de l'année scolaire de référence. La réponse est déclarative et ne dit rien sur la période totale de participation scolaire au cours de cette année de référence. Toutefois, il convient de mentionner que les résultats des enquêtes auprès des ménages donnent des ordres de grandeur de la participation scolaire assez proches des statistiques scolaires ${ }^{6}$.

- Au niveau de la mesure de l'absentéisme, le choix de deux semaines minimum comme durée d'absence répondait au souci de limiter le biais de mémoire pour les enquêtés de se rappeler toutes les absences, même celles de très courtes durée (moins d'une semaine). Mais finalement, les résultats suggèrent que les absences au cours de l'année scolaire de quatre semaines au moins sont rares. On peut donc supposer qu'une mesure plus fine de l'absence, peut-être au minimum deux semaines ou une semaine de durée d'absence, aurait permis de capter davantage les absences pour motif de non paiement de frais scolaires.

\section{Au niveau de l'enquête qualitative :}

- L'enquête qualitative n'a pas porté sur l'ensemble des 11 provinces pour des raisons de ressources limitées. La décision faite a donc été de choisir 4 provinces ayant des profils socio-économiques diversifiés par rapport à la problématique des EADE afin de voir s'il y a convergence ou divergence des discours sur ce phénomène des EADE. Avec plus de ressources, on aurait pu élargir les entretiens aux autres provinces, mais les résultats du qualitatif ne révèlent pas une différence entre les 4 provinces en termes de discours.

- L'un des objectifs de l'enquête qualitative était d'avoir le point de vue des différents acteurs du système éducatif. En ce qui concerne la structure par sexe du personnel enseignant et responsables du système éducatif, les statistiques scolaires révèlent qu'on a $30 \%$ de femmes et $70 \%$ des hommes. Dans la démarche de la collecte des données qualitatives, le respect scrupuleux de cette structure par sexe n'a pas été un critère pour sélectionner la cible des responsables du système éducatif. Par ailleurs, le nombre de femmes parents interviewés est nettement inférieur à celui des hommes parents, en raison de la structure sociale qui fait que c'est l'homme qui est presque toujours interpelé sur les questions du ménage ou de la famille. De plus, il n'y a pas eu au départ une répartition des parents à interviewer en termes de sexe.

\footnotetext{
${ }^{6}$ Si le dénominateur (les effectifs de population scolarisable) utilisé dans les statistiques scolaires est actualisé et relativement proche des données de l'enquête ménage. Les enquêtes ménages ont en fait l'avantage de donner aussi bien le numérateur que le dénominateur à partir de la même source.
} 


\section{Profil des EADE}

\subsection{Statut scolaire des enfants}

La structure de la population scolarisable (5-17 ans) montre que pour l'ensemble du pays, plus de la moitié de la population scolarisable estimée à 25455279 , est âgée de 6-11 ans (13 117697$)$, soit 51,5\%. Les enfants âgés de 14-17 ans viennent en deuxième position avec un effectif absolu de 5701982 (22,4\%). Viennent ensuite les 12-13 ans avec un effectif absolu de 3919945 (soit 14,4\%) et enfin la population de 5 ans avec un effectif de 2720081 (soit 10,7\%). Cette structure est quasiment la même selon le milieu de résidence et le sexe (tableau 10).

Tableau 10 : Répartition (en \%) de la population scolarisable par groupes d'âges selon le sexe et le milieu de résidence

\begin{tabular}{|c|c|c|c|c|c|c|c|c|c|}
\hline \multirow{2}{*}{$\begin{array}{l}\text { Groupes } \\
\text { d'âges }\end{array}$} & \multicolumn{3}{|l|}{ Urbain } & \multicolumn{3}{|l|}{ Rural } & \multicolumn{3}{|l|}{ Ensemble } \\
\hline & Garçons & Filles & Ensemble & Garçons & Filles & Ensemble & Garçons & Filles & Ensemble \\
\hline 5 ans & 8,5 & 9,7 & 9,1 & 11,4 & 11,6 & 11,5 & 10,5 & 10,9 & 10,7 \\
\hline 6-11 ans & 50,3 & 49,8 & 50,0 & 52,2 & 52,3 & 52,3 & 51,6 & 51,4 & 51,5 \\
\hline $12-13$ ans & 16,0 & 13,6 & 14,8 & 15,3 & 16,0 & 15,7 & 15,6 & 15,2 & 15,4 \\
\hline $14-17$ ans & 25,2 & 26,9 & 26,1 & 21,0 & 20,1 & 20,6 & 22,4 & 22,4 & 22,4 \\
\hline Total & 100,0 & 100,0 & 100,0 & 100,0 & 100,0 & 100,0 & 100,0 & 100,0 & 100,0 \\
\hline Effectifs & 4193295 & 4205790 & 8399085 & 8965230 & 8090964 & 17056194 & 13158525 & 12296754 & 25455279 \\
\hline
\end{tabular}

Source : Données de l'enquête auprès des ménages, EADE-RDC 2012

A la différence des autres enquêtes qui nécessitent le rapprochement de plusieurs informations pour élaborer le profil scolaire, l'enquête EADE le saisit directement. II permet une distinction nette du statut scolaire et couvre une gamme plus large des tranches d'âges à savoir les 3-17 ans. Cependant, pour les besoins de comparaisons, le profil scolaire sera décrit pour les 5-17 ans tout comme dans l'état des lieux. Trois catégories d'enfants sont distinguées dans le statut scolaire :

- les enfants qui fréquentent l'école au moment de l'enquête (ou enfants scolarisés) ;

- les enfants qui ont déjà fréquenté l'école et ne fréquentant pas l'école au moment de l'enquête (ou enfants déscolarisés ou encore les abandons) ;

- les enfants qui n'ont jamais fréquenté l'école au moment de l'enquête.

\subsubsection{Distribution des enfants et adolescents par statut scolaire selon l'âge et le sexe}

L'EADE 2012 révèle que 71,1\% des enfants de 5-17 ans fréquentaient l'école en 2011-2012 (tableau 11). Ce pourcentage est le plus élevé chez les $12-13$ ans (86,9\%), suivi des $14-17$ ans (78,8 \%), des 6-11 ans $(73,2$ $\%)$ et des 5 ans $(21,2 \%)$.

La proportion des 5-17 ans en dehors de l'école est estimée à 28,9\% par l'EADE 2012, soit en termes absolus, 7375876 enfants et adolescents ${ }^{7}$ (tableau 11). Parmi ces enfants en dehors de l'école, les filles représentent plus de la moitié soit 3892464 (52,7\%). C'est aussi chez les filles que l'ampleur du phénomène des enfants et adolescents en dehors de l'école est la plus forte : $31,7 \%$ chez les filles contre $26,5 \%$ chez les garçons.

C'est chez les 5 ans que la proportion des enfants en dehors de l'école est la plus élevée (78,8\%), suivi des 6-11 ans avec une proportion d'EADE de 26,7 \%, des 14-17 ans avec une proportion de 21,2\% d'EADE et enfin les 12-13 ans avec une proportion d'EADE de 13,1\% (pour les données par année d'âge, cf. tableaux A2, A3 et A4 en annexe).

L'examen des données du tableau 11, fait ressortir une différence assez notable entre sexes. En effet, 73,5\% des garçons sont à l'école contre $68,3 \%$ de filles. Les filles accèdent moins à l'école $(23,5 \%$ d'entre elles n'ont

\footnotetext{
${ }^{7}$ L'état des lieux sur la base du MICS 2010 donnait un effectif de 7655592 (une différence de 279717 avec EADE 2012).
} 
jamais fréquenté l'école) que les garçons (20,9\%). En outre, il y a plus d'abandons chez les filles $(8,0 \%$ contre $5,6 \%$ chez les garçons).

Tableau 11 : Statut scolaire des enfants de 5-17 ans par groupe d'âges et sexe (en \%)

\begin{tabular}{|c|c|c|c|c|c|c|c|}
\hline \multirow[b]{2}{*}{ Caractéristiques } & \multicolumn{5}{|c|}{ Statut scolaire } & \multicolumn{2}{|c|}{ EADE } \\
\hline & Scolarisé & Déscolarisé & $\begin{array}{c}\text { Jamais } \\
\text { fréquenté }\end{array}$ & Total & Effectifs & $\%$ & Effectifs \\
\hline \multicolumn{8}{|l|}{ Garçons } \\
\hline 5 ans & 20,7 & 0,6 & 78,7 & 100,0 & 1376624 & 79,3 & 1091943 \\
\hline 6-11 ans & 74,2 & 4,0 & 21,7 & 100,0 & 6791505 & 25,7 & 1749792 \\
\hline $12-13$ ans & 90,1 & 5,9 & 4,1 & 100,0 & 2047336 & 10,0 & 203408 \\
\hline $14-17$ ans & 85,1 & 11,3 & 3,6 & 100,0 & 2943062 & 14,9 & 438269 \\
\hline Total & 73,5 & 5,6 & 20,9 & 100,0 & 13158527 & 26,5 & 3483412 \\
\hline \multicolumn{8}{|l|}{ Filles } \\
\hline 5 ans & 21,7 & 0,4 & 77,9 & 100,0 & 1343459 & 78,3 & 1052252 \\
\hline 6-11 ans & 72,2 & 4,4 & 23,4 & 100,0 & 6326091 & 27,8 & 1759459 \\
\hline $12-13$ ans & 83,4 & 8,3 & 8,2 & 100,0 & 1870610 & 16,5 & 309760 \\
\hline $14-17$ ans & 72,0 & 20,3 & 7,6 & 100,0 & 2756594 & 27,9 & 770993 \\
\hline Total & 68,3 & 8,2 & 23,5 & 100,0 & 12296754 & 31,7 & 3892464 \\
\hline \multicolumn{8}{|l|}{ Ensemble } \\
\hline 5 ans & 21,2 & 0,5 & 78,3 & 100,0 & 2720083 & 78,8 & 2144195 \\
\hline 6-11 ans & 73,2 & 4,2 & 22,5 & 100,0 & 13117596 & 26,7 & 3509251 \\
\hline $12-13$ ans & 86,9 & 7,0 & 6,1 & 100,0 & 3917946 & 13,1 & 513168 \\
\hline $14-17$ ans & 78,8 & 15,7 & 5,5 & 100,0 & 5699656 & 21,2 & 1209262 \\
\hline Total & 71,1 & 6,8 & 22,1 & 100,0 & 25455281 & 28,9 & 7375876 \\
\hline
\end{tabular}

Source : Données de l'enquête auprès des ménages, EADE-RDC 2012

La parité entre sexes (taux des filles/taux des garçons) en matière de fréquentation scolaire des 5-17 ans est de 0,93 à travers l'enquête EADE 2012. Cette parité entre sexes est encore plus proche de un (1) au niveau de la tranche d'âge du primaire : en effet, 72,2 \% des filles de 6-11 ans sont à l'école en RDC en 2011-2012 contre $74,3 \%$ des garçons, soit un indice de parité de 0,97. Ce chiffre est en nette amélioration par rapport à ce qui a été observé à travers les enquêtes passées (EDS 2007 et MICS 2010) : en effet, pour les 6-11 ans en 2007, les taux de fréquentation scolaire étaient respectivement de $59,3 \%$ et de $62,6 \%$ pour les filles et les garçons, soit un indice de parité de 0,94. En 2010, les taux de fréquentation scolaire à 6-11 ans étaient respectivement de $68,3 \%$ et de $73,2 \%$ pour les filles et les garçons, soit un indice de parité de $0,93^{8}$. Cette réduction importante des écarts entre sexes en RDC, notamment au niveau du primaire, est, comme cela ressort des entretiens qualitatifs avec les acteurs du système éducatif, des actions de sensibilisation menées ainsi que le processus de pacification et de reprise économique après le conflit des années 2006. Nous y revenons plus loin dans l'analyse des opinions des acteurs du système éducatif sur l'appréciation des inégalités scolaires. La tendance des écarts entre sexes en RDC est aussi conforme à ce qui est observé au niveau mondial et notamment en Afrique. En effet, comme il ressort du rapport mondial de suivi de l'EPT de 2012, "la réduction de l'écart entre sexes en matière de scolarisation dans l'enseignement primaire est l'un des plus grands succès de l'EPT » (UNESCO, 2012, p. 7). Alors que 33 pays (sur 167 disposant de données) dont 17 en Afrique subsaharienne, avaient un indice de parité des sexes au primaire inférieur à 0,90 en 1999, en 2010, ce groupe ne compte plus que 17 pays dont 12 en Afrique subsaharienne (UNESCO, 2012).

L'évolution de la proportion des enfants et adolescents en dehors de l'école (graphique 2) montre une baisse régulière depuis 2007, passant de 38,5\% en 2007 (EDS 2007), à 32,5\% en 2010 (MICS 2010) et 28,9 \% en 2012 (EADE 2012) ${ }^{9}$.

\footnotetext{
${ }^{8}$ L'annuaire statistique 2010-2011 de la DEP-EPSP (le plus récent) ne donne pas les taux nets de scolarisation, mais plutôt des taux bruts de scolarisation au primaire, ce qui rend la comparaison difficile avec un taux de fréquentation scolaire des 6 11 ans dans les enquêtes auprès des ménages. Mais on peut mentionner que, l'un indice de parité pour le taux de brut de scolarisation au primaire selon l'annuaire 2010-2011 est de 0,86 (TBS fille de 85,8 \% et TBS garçon de 99,6\%).

${ }^{9}$ Le calcul de l'intervalle de confiance à $95 \%$ de la différence entre les proportions des EADE des années successives (Annexe 2), révèle que les écarts observés entre 2007, 2010 et 2012 sont statistiquement significatifs.
} 
Au cours de la période (2007-2012), le rythme de baisse moyen de la proportion des EADE a été de 1,92\% par an. Si cette tendance se poursuit, c'est en 2027, donc dans 15 ans qu'on pourrait espérer avoir tous les enfants et adolescents à l'école en RDC.

Sur la même période de cinq ans (2007-2012), la baisse relative ${ }^{10}$ de la proportion des EADE a été la plus importante dans la tranche d'âge des $12-13$ ans (33,5\% de baisse), suivie de la tranche d'âges des 6-11 ans $(31,5 \%)$, de celle des $14-17$ ans $(27,6 \%)$. Elle a été la plus faible chez les 5 ans (14,2\%).

Graphique 2 : Evolution de la proportion (en \%) des enfants et adolescents en dehors de l'école selon la tranche d'âges

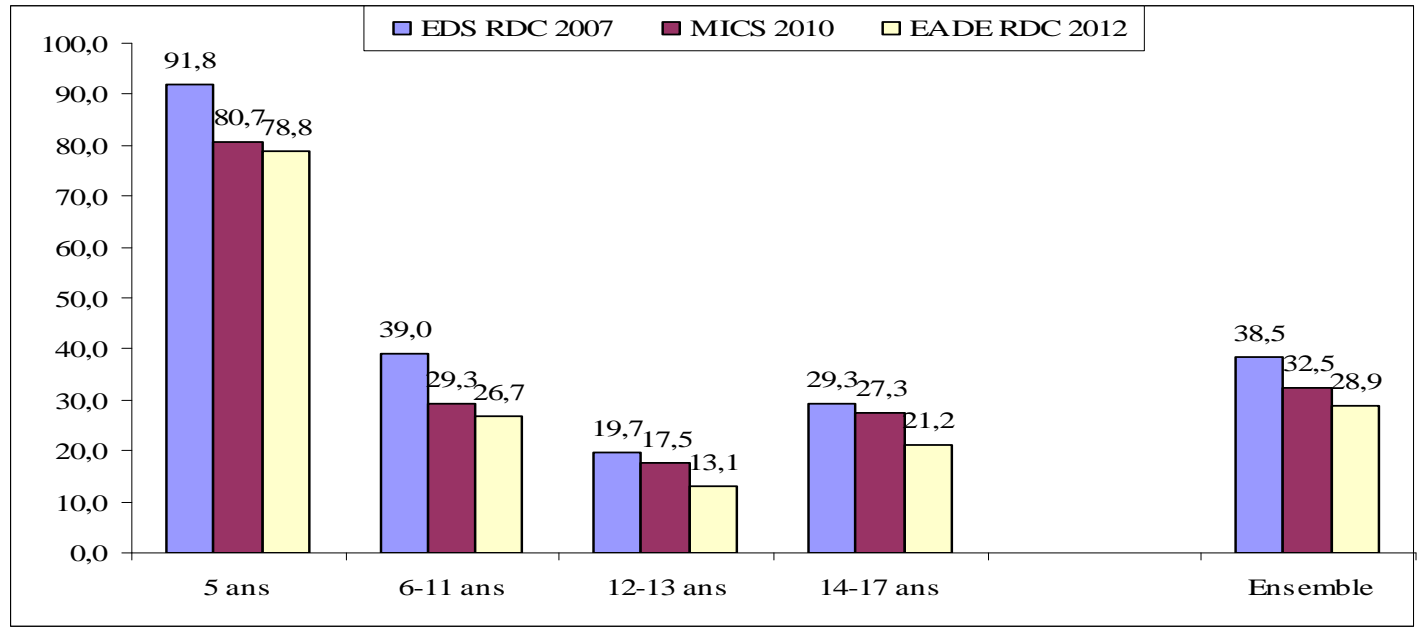

Source : Données de l'enquête auprès des ménages, EADE-RDC 2012, MICS-RDC 2010, EDS-RDC 2007

\subsubsection{Distribution des enfants et adolescents par statut scolaire selon le milieu de résidence}

\section{a) Urbain versus rural}

Le phénomène des enfants en dehors de l'école est plus prépondérant en milieu rural $(33,4 \%)$ qu'en milieu urbain (20,0\%). En termes absolus, ce sont 5694525 EADE en milieu rural contre 1681391 en milieu urbain (tableau 12). C'est au niveau de l'accès que les écarts sont importants entre la ville et la campagne : en effet, $27,1 \%$ des enfants n'ont jamais fréquenté en milieu rural contre seulement $12,1 \%$ en milieu urbain. Les déscolarisés sont proportionnellement plus importants à la campagne $(7,9 \%)$ qu'en ville $(6,3 \%)$. C'est au niveau de la structure par âge des déscolarisés que les différences sont nettes. A la campagne, les déscolarisés sont particulièrement en nombre important aux âges 12-13 ans et 14-17 ans. Le travail des enfants, beaucoup plus précoce et plus important en milieu rural pourrait être à l'origine de ces écarts.

${ }^{10}$ La baisse relative est égale à la baisse absolue (différence entre la proportion des EADE en 2007 et la proportion des EADE en 2012) rapportée à la proportion des EADE en 2007. Cet indicateur permet de mieux rendre compte des progrès par rapport au niveau initial (en 2007), notamment dans une perspective comparative. 
Tableau 12 : Statut scolaire des enfants de 5-17 ans par groupe d'âges et milieu de résidence (en \%)

\begin{tabular}{|c|c|c|c|c|c|c|c|}
\hline \multirow[b]{2}{*}{ Caractéristiques } & \multicolumn{5}{|c|}{ Statut scolaire } & \multicolumn{2}{|c|}{ EADE } \\
\hline & Scolarisé & Déscolarisé & $\begin{array}{c}\text { Jamais } \\
\text { fréquenté }\end{array}$ & Total & Effectifs & $\%$ & Effectifs \\
\hline \multicolumn{8}{|l|}{ Urbain } \\
\hline 5 ans & 40,4 & 1,3 & 58,3 & 100,0 & 762974 & 59,6 & 454494 \\
\hline 6-11 ans & 81,9 & 5,8 & 12,2 & 100,0 & 4202049 & 18,0 & 760389 \\
\hline $12-13$ ans & 90,5 & 6,9 & 2,7 & 100,0 & 1245718 & 9,6 & 118960 \\
\hline $14-17$ ans & 84,1 & 14,6 & 1,2 & 100,0 & 2188346 & 15,8 & 347508 \\
\hline Total & 80,0 & 7,9 & 12,1 & 100,0 & 8399087 & 20,0 & 1681351 \\
\hline \multicolumn{8}{|l|}{ Rural } \\
\hline 5 ans & 13,7 & 0,2 & 86,1 & 100,0 & 1957109 & 86,3 & 1689701 \\
\hline 6-11 ans & 69,2 & 3,5 & 27,4 & 100,0 & 8915547 & 30,9 & 2748862 \\
\hline $12-13$ ans & 85,2 & 7,1 & 7,6 & 100,0 & 2672228 & 14,7 & 394208 \\
\hline $14-17$ ans & 75,5 & 16,3 & 8,2 & 100,0 & 3511310 & 24,5 & 861754 \\
\hline Total & 66,6 & 6,3 & 27,1 & 100,0 & 17056194 & 33,4 & 5694525 \\
\hline \multicolumn{8}{|l|}{ Ensemble } \\
\hline 5 ans & 21,2 & 0,5 & 78,3 & 100,0 & 2720083 & 78,8 & 2144195 \\
\hline 6-11 ans & 73,2 & 4,2 & 22,5 & 100,0 & 13117596 & 26,7 & 3509251 \\
\hline $12-13$ ans & 86,9 & 7,0 & 6,1 & 100,0 & 3917946 & 13,1 & 513168 \\
\hline $14-17$ ans & 78,8 & 15,7 & 5,5 & 100,0 & 5699656 & 21,2 & 1209262 \\
\hline Total & 71,1 & 6,8 & 22,1 & 100,0 & 25455281 & 28,9 & 7375876 \\
\hline
\end{tabular}

Source : Données de l'enquête auprès des ménages, EADE-RDC 2012

\section{b) Comparaison des provinces}

Les graphiques 3 et 4 nous donnent visuellement la situation géographique de l'ampleur (en termes aussi bien relatifs qu'absolus) du phénomène des EADE des 5-17 ans et des 6-11 ans. En considérant les 5-17 ans, il ressort que c'est au Nord Kivu qu'il y a proportionnellement plus d'enfants d'âges scolaires en dehors de l'école, soit 43,9\% (tableau A5 en annexe). Cinq autres provinces ont des proportions d'enfants en dehors de l'école supérieures à la moyenne nationale qui est de $28,9 \%$. II s'agit du Katanga (34,8 \%), du Kassaï occidental (32,4\%), de la province orientale (32,2\%), du Sud-Kivu (30,3\%) et du Kassaï oriental (29,3\%). En termes absolus, c'est le Katanga qui vient en tête avec 1334876 enfants en dehors de l'école (graphique 3), suivi de la Province orientale (1 039 858), du Nord Kivu (994 366) et de l'Equateur (726 194). Les mêmes variations provinciales observées au niveau des 5-17 ans se maintiennent au niveau des 6-11 ans. On peut toutefois noter l'importance de la distinction qu'il y à faire entre l'ampleur en termes relatifs et celle en termes absolus : en effet, la province de Kinshasa et celle du Bandundu sont celles où le phénomène a une ampleur relative faible, mais en termes absolus, il y a plus d'EADE à Kinshasa et dans le Bandundu comparativement au Maniema. 
Graphique 3 : Carte des proportions (en \%) et des effectifs des EADE de 5-17 ans par province, EADE-RDC 2012

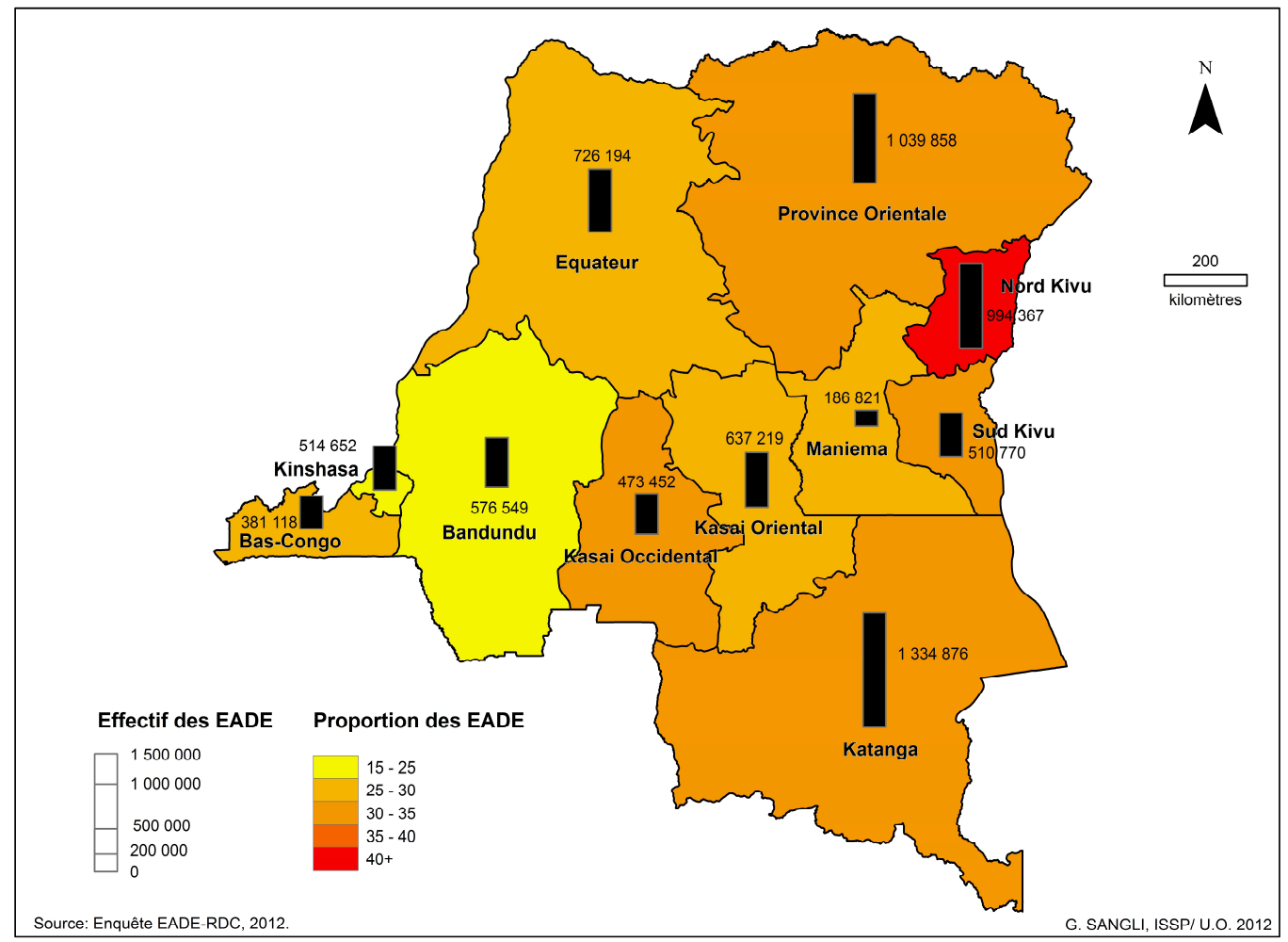

Source : Données de l'enquête auprès des ménages, EADE-RDC 2012

Graphique 4 : Carte des proportions (en \%) et des effectifs des EADE de 6-11 ans par province, EADE-RDC 2012

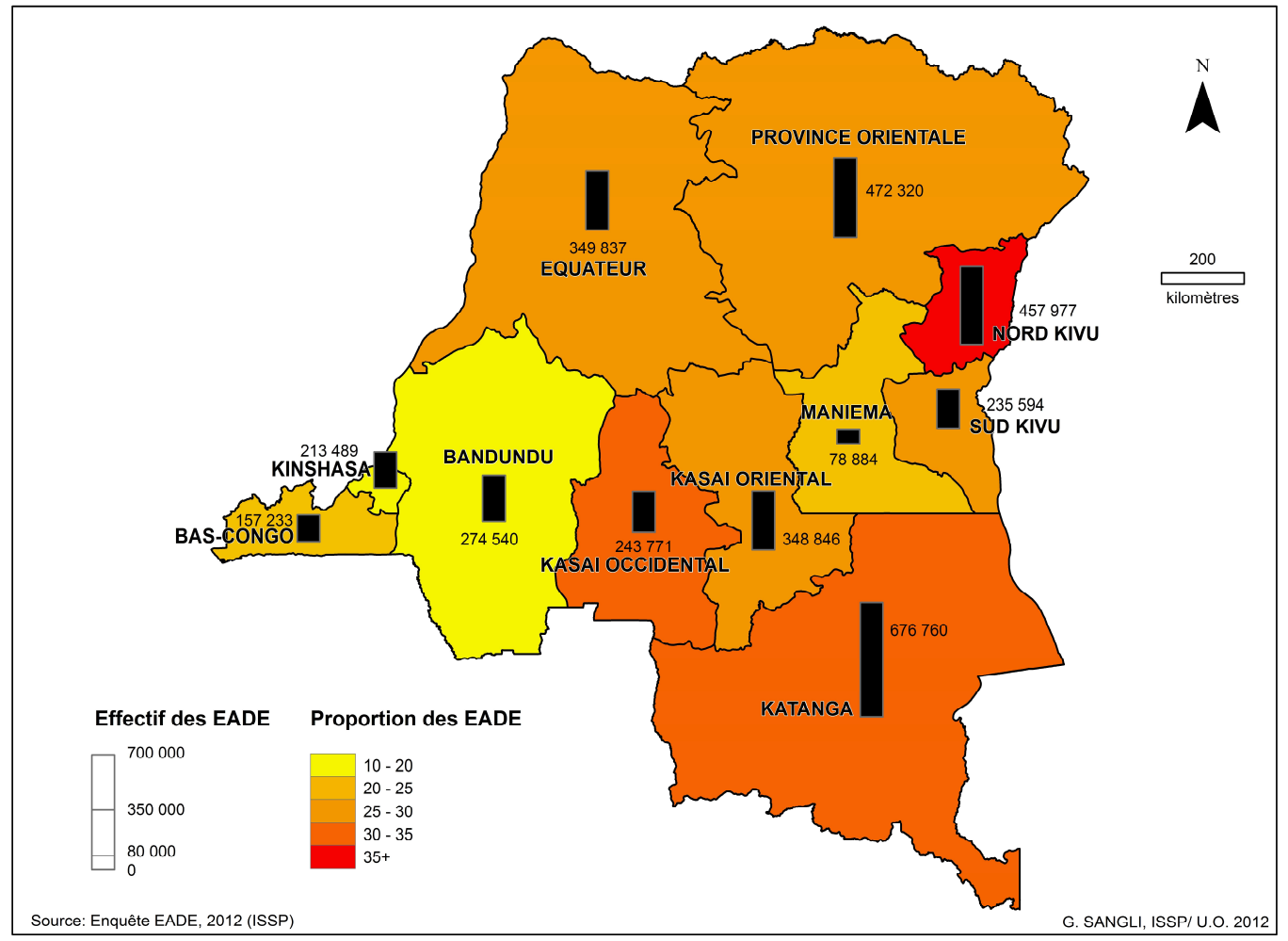

Source : Données de l'enquête auprès des ménages, EADE-RDC 2012

Quant au phénomène de la déscolarisation, c'est au Nord Kivu qu'il est le plus important $(9,7 \%)$ suivi de la province orientale $(9,0 \%)$, de Kinshasa $(8,6 \%)$, du Katanga (7,8 \%), du Kasaï Oriental $(7,5 \%)$ et du Sud-Kivu $(7,2 \%)$ (tableau A5 en annexe). 
L'examen de l'évolution par province (Graphique 5) révèle que c'est dans la province du Bas Congo que la baisse da la proportion des EADE en termes relatifs a été plus importante $(40,9 \%$ de baisse), suivie de Kinshasa (39,6\%) et du Bandundu (36,5\%). A l'opposé, le Katanga qui a connu une hausse du phénomène en 2010, connaît une baisse relativement faible sur la période des cinq ans (9,0\%). Cependant, la baisse relative la plus faible est observée dans la Province orientale $(6,2 \%)$.

Graphique 5 : Evolution de la proportion (en \%) des enfants de 5-17 ans en dehors de l'école par province

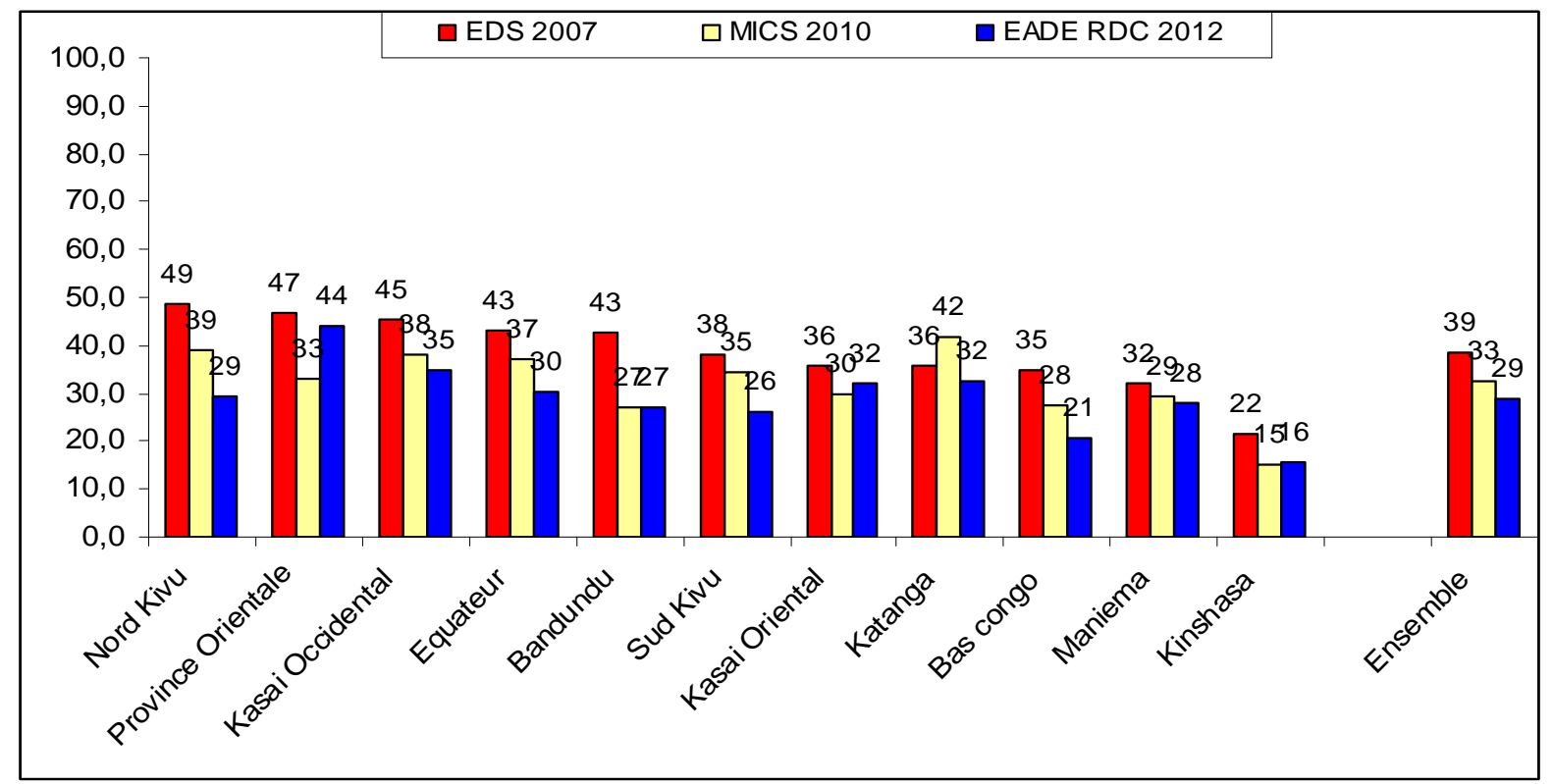

Source : Données de l'enquête auprès des ménages, EADE-RDC 2012, MICS-RDC 2010, EDS-RDC 2007

Exceptée la ville province de Kinshasa (où il n'y a pas de différence entre garçons et filles), dans toutes les autres provinces, les filles sont proportionnellement plus nombreuses à être en dehors de l'école comparées aux garçons (graphique 6). C'est dans le Kasaï Oriental et le Kasaï Occidental que l'écart entre garçons et filles est la plus forte. Les écarts entre sexes sont par contre faibles dans le Bandundu, le Maniema et la Province Orientale.

Graphique 6 : Proportion (en \%) d'enfants de 5-17 ans en dehors de l'école par sexe et selon la province

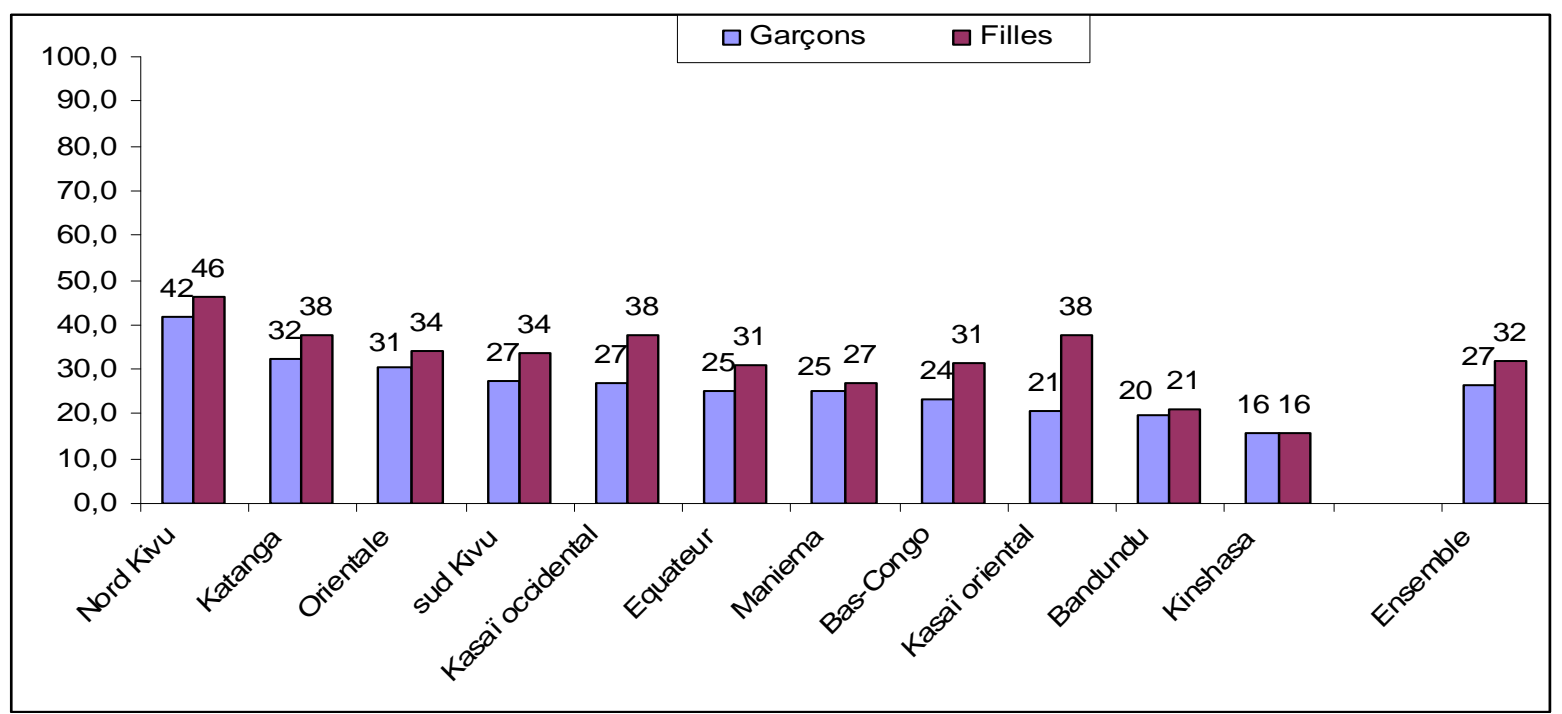

Source : Données de l'enquête auprès des ménages, EADE-RDC 2012. Graphique construit à partir du tableau A5 en annexe

Le graphique 7 montre la participation scolaire des 5-17 ans pour chaque niveau d'enseignement (préprimaire, primaire, premier cycle du secondaire et deuxième cycle du secondaire). II ressort, comme mentionné plus haut, que la participation au pré-primaire est très faible et qu'une petite proportion d'enfants de 6 ans (et même de 7 ans) fréquente le pré-primaire (aire de couleur bleue). La participation scolaire est plus 
élevée au primaire (aire de couleur verte). Mais bien que la tranche d'âges officiels soit 6-11 pour le primaire, on note qu'une bonne proportion d'enfants d'âges du supérieur (jusqu'à 17 ans) fréquentent toujours le primaire. Par ailleurs, moins de $50 \%$ des enfants de 6 ans participent à l'école et même à 7 ans, ils sont moins de $70 \%$, ce qui reflète assez bien le phénomène des retards scolaires sur lesquels nous reviendrons. Près de $13 \%$ d'enfants de 5 ans fréquentent le primaire. Le phénomène du retard scolaire s'observe également lorsqu'on examine les autres niveaux d'enseignement.

Graphique 7 : Participation scolaire par âge et niveau d'études des enfants et adolescents de 5-17 ans en 2012 (en \%)

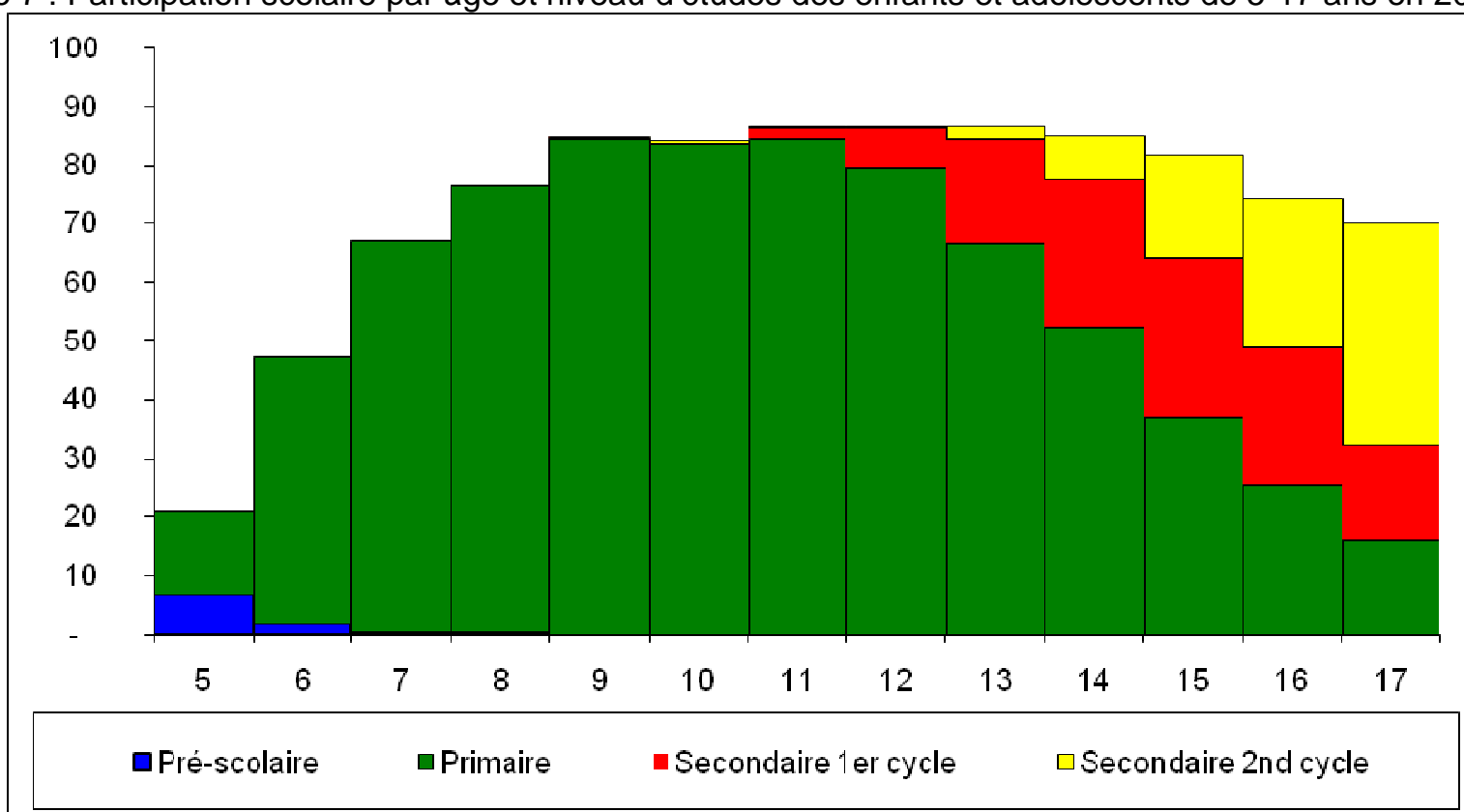

Source : Données de l'enquête auprès des ménages, EADE-RDC 2012 


\subsubsection{Statut scolaire des enfants et adolescents dans les structures et dans la rue}

a) Enfants et adolescents dans les structures d'accueil

Les enfants et adolescents qui sont dans les structures d'accueil fréquentent dans leur grande majorité $(83,8$ \%) (tableau 13).

Tableau 13 : Statut scolaire des enfants de 5-17 ans dans les structures d'accueil par groupe d'âges (en \%)

\begin{tabular}{|c|c|c|c|c|c|c|c|}
\hline \multirow[b]{2}{*}{ Caractéristiques } & \multicolumn{5}{|c|}{ Statut scolaire } & \multicolumn{2}{|c|}{ EADE } \\
\hline & Scolarisé & Déscolarisé & $\begin{array}{l}\text { Jamais } \\
\text { fréquenté }\end{array}$ & Total & Effectifs & $\%$ & Effectifs \\
\hline \multicolumn{8}{|l|}{ Garçons } \\
\hline 5 ans & 65,4 & 2,3 & 32,3 & 100,0 & 130 & 34,6 & 45 \\
\hline 6-11 ans & 87,4 & 5,5 & 7,1 & 100,0 & 2334 & 12,6 & 294 \\
\hline $12-13$ ans & 84,9 & 10,8 & 4,3 & 100,0 & 1450 & 15,1 & 219 \\
\hline $14-17$ ans & 78,1 & 16,4 & 5,4 & 100,0 & 2131 & 21,9 & 466 \\
\hline Ens $\mathrm{G}$ & 83,1 & 10,6 & 6,4 & 100,0 & 6045 & 16,9 & 1024 \\
\hline \multicolumn{8}{|l|}{ Filles } \\
\hline 5 ans & 68,2 & 0,7 & 31,1 & 100,0 & 148 & 31,8 & 47 \\
\hline 6-11 ans & 90,7 & 3,7 & 5,6 & 100,0 & 2069 & 9,3 & 192 \\
\hline $12-13$ ans & 90,4 & 6,6 & 3,0 & 100,0 & 1133 & 9,6 & 109 \\
\hline $14-17$ ans & 76,3 & 17,9 & 5,9 & 100,0 & 1863 & 23,7 & 442 \\
\hline Ens $\mathrm{F}$ & 84,8 & 9,3 & 5,8 & 100,0 & 5213 & 15,2 & 790 \\
\hline \multicolumn{8}{|l|}{$\mathrm{G}+\mathrm{F}$} \\
\hline 5 ans & 66,9 & 1,4 & 31,7 & 100,0 & 278 & 33,1 & 92 \\
\hline 6-11 ans & 89,0 & 4,7 & 6,4 & 100,0 & 4403 & 11,0 & 486 \\
\hline $12-13$ ans & 87,3 & 8,9 & 3,8 & 100,0 & 2583 & 12,7 & 328 \\
\hline $14-17$ ans & 77,3 & 17,1 & 5,6 & 100,0 & 3994 & 22,7 & 908 \\
\hline Ens $\mathrm{G}+\mathrm{F}$ & 83,9 & 10,0 & 6,1 & 100,0 & 11258 & 16,1 & 1814 \\
\hline
\end{tabular}

Source : Données du recensement auprès des structures d'accueil des enfants, EADE-RDC 2012

b) Enfants vivant dans la rue

La plupart des enfants vivant dans la rue sont des déscolarisés ${ }^{11}$ (tableau 14). On note tout de même que 4,6 $\%$ des enfants vivant dans la rue sont scolarisés. II n'y a pas de différence entre filles et garçons en matière de scolarisation des enfants vivant dans la rue.

\footnotetext{
${ }^{11}$ Les abandons scolaires des enfants vivant dans la rue se font principalement au primaire.
} 
Tableau 14 : Statut scolaire des enfants de 5-17 ans de la rue par groupe d'âges (en \%)

\begin{tabular}{|c|c|c|c|c|c|c|c|}
\hline \multirow[b]{2}{*}{ Caractéristiques } & \multicolumn{5}{|c|}{ Statut scolaire } & \multicolumn{2}{|c|}{ EADE } \\
\hline & Scolarisé & Déscolarisé & $\begin{array}{c}\text { Jamais } \\
\text { fréquenté }\end{array}$ & Total & Effectifs & $\%$ & Effectifs \\
\hline \multicolumn{8}{|l|}{ Garçons } \\
\hline 5 ans & 0,0 & 0,0 & 100,0 & 100,0 & 36 & 100,0 & 36 \\
\hline 6-11 ans & 3,8 & 37,9 & 58,3 & 100,0 & 900 & 96,2 & 866 \\
\hline $12-13$ ans & 6,7 & 60,5 & 32,8 & 100,0 & 780 & 93,3 & 728 \\
\hline $14-17$ ans & 3,7 & 69,7 & 26,7 & 100,0 & 1846 & 96,3 & 1778 \\
\hline Ens G & 4,3 & 58,9 & 36,7 & 100,0 & 3562 & 95,7 & 3408 \\
\hline \multicolumn{8}{|l|}{ Filles } \\
\hline 5 ans & 0,0 & 0,0 & 100,0 & 100,0 & 7 & 100,0 & 7 \\
\hline 6-11 ans & 6,3 & 40,0 & 53,8 & 100,0 & 240 & 93,8 & 225 \\
\hline $12-13$ ans & 6,8 & 58,4 & 34,7 & 100,0 & 190 & 93,2 & 177 \\
\hline $14-17$ ans & 5,1 & 72,6 & 22,2 & 100,0 & 351 & 94,9 & 333 \\
\hline Ens $\mathrm{F}$ & 5,8 & 58,6 & 35,5 & 100,0 & 788 & 94,2 & 742 \\
\hline \multicolumn{8}{|l|}{$\mathrm{G}+\mathrm{F}$} \\
\hline 5 ans & 0,0 & 0,0 & 100,0 & 100,0 & 43 & 100,0 & 43 \\
\hline 6-11 ans & 4,3 & 38,3 & 57,4 & 100,0 & 1140 & 95,7 & 1091 \\
\hline $12-13$ ans & 6,7 & 60,1 & 33,2 & 100,0 & 970 & 93,3 & 905 \\
\hline $14-17$ ans & 3,9 & 70,1 & 25,9 & 100,0 & 2197 & 96,1 & 2111 \\
\hline Ens $\mathrm{G}+\mathrm{F}$ & 4,6 & 58,9 & 36,5 & 100,0 & 4350 & 95,4 & 4150 \\
\hline
\end{tabular}

Source : Données de l'enquête auprès des enfants de la rue, EADE-RDC 2012

\subsection{Profil des enfants en dehors de l'école d'âges du pré-primaire (Dimension 1)}

Si dans les enquêtes auprès des ménages (MICS et EDS), la question sur la fréquentation scolaire des enfants d'âges du pré-primaire n'est posée qu'à ceux âgés de 5 ans, l'enquête EADE RDC de 2012 a investigué la fréquentation scolaire des enfants âgés de 3 à 5 ans. Les proportions calculées portent donc sur ces enfants d'âges du pré-primaire, avec toutefois un focus sur les enfants de 5 ans pour des perspectives de comparaison internationale. L'objectif de cette section est de donner le profil des EADE, c'est-à-dire de décrire leurs caractéristiques socio-démographiques et montrer dans quels types de ménage ils se retrouvent davantage. II ne s'agit pas ici d'expliquer pourquoi ils sont EADE, cet exercice interviendra dans le chapitre 3 sur l'analyse des barrières et déterminants de l'exclusion scolaire.

\subsubsection{Sexe de l'enfant et répartition spatiale des enfants en dehors de l'école de 3-5 ans}

Les enfants en dehors de l'école âgés de 3-5 ans représentent un effectif de 6699099 individus. Ceux âgés de 5 ans sont au nombre de 2144194 individus soit 32,0 \% de la population d'âges du pré-primaire.

Parmi les enfants en dehors de l'école de 3-5 ans, il y a 51,6 \% de garçons et $48,4 \%$ de filles. La répartition par sexe des enfants de 3-5 ans qui n'ont jamais fréquenté l'école reste à l'avantage des filles car elles ne représentent que $48,5 \%$ (tableau 15). La tendance est presque la même pour les déscolarisés, mais il y a plus de garçons déscolarisés $(58,0 \%)$ que de filles $(42,0 \%)$. Ainsi, même si les garçons sont plus scolarisés que les filles, c'est parmi eux que l'on retrouve proportionnellement le plus de déscolarisés. 
Tableau 15 : Répartition (en \%) des enfants d'âges du pré-primaire en dehors de l'école par statut scolaire selon le sexe de l'enfant, le milieu de résidence et la province

\begin{tabular}{|c|c|c|c|c|c|c|}
\hline \multirow[b]{2}{*}{ Caractéristiques } & \multicolumn{3}{|c|}{ Enfants de $3-5$ ans } & \multicolumn{3}{|c|}{ Enfants de 5 ans } \\
\hline & Déscolarisé & $\begin{array}{c}\text { Jamais } \\
\text { fréquenté }\end{array}$ & Ens. & Déscolarisé & $\begin{array}{l}\text { Jamais } \\
\text { fréquenté }\end{array}$ & Ens. \\
\hline \multicolumn{7}{|l|}{ Sexe de l'enfant } \\
\hline Garçons & 58,0 & 51,5 & 51,6 & 59,3 & 50,9 & 50,9 \\
\hline Filles & 42,0 & 48,5 & 48,4 & 40,7 & 49,1 & 49,1 \\
\hline Total & 100,0 & 100,0 & 100,0 & 100,0 & 100,0 & 100,0 \\
\hline \multicolumn{7}{|l|}{ Milieu de résidence } \\
\hline Urbain & 64,3 & 25,3 & 25,5 & 72,9 & 20,9 & 21,2 \\
\hline Rural & 35,7 & 74,7 & 74,5 & 27,1 & 79,1 & 78,8 \\
\hline Total & 100,0 & 100,0 & 100,0 & 100,0 & 100,0 & 100,0 \\
\hline \multicolumn{7}{|l|}{ Provinces } \\
\hline Kinshasa & 25,6 & 8,2 & 8,2 & 32,2 & 6,0 & 6,2 \\
\hline Bas-Congo & 9,9 & 6,4 & 6,4 & 13,2 & 6,3 & 6,3 \\
\hline Bandundu & 1,3 & 11,7 & 11,7 & - & 11,0 & 10,9 \\
\hline Equateur & 13,9 & 11,3 & 11,3 & 18,8 & 11,7 & 11,7 \\
\hline Orientale & 12,6 & 10,0 & 10,0 & - & 13,7 & 13,6 \\
\hline Nord Kivu & 1,3 & 11,0 & 11,0 & 1,7 & 12,7 & 12,6 \\
\hline Maniema & 3,6 & 3,0 & 3,0 & 3,3 & 3,6 & 3,6 \\
\hline sud Kivu & 5,1 & 7,7 & 7,6 & - & 6,8 & 6,7 \\
\hline Katanga & 14,8 & 16,9 & 16,8 & 23,8 & 14,8 & 14,8 \\
\hline Kasaï oriental & 5,6 & 7,4 & 7,4 & 7,1 & 6,7 & 6,7 \\
\hline Kasaï occidental & 6,4 & 6,5 & 6,5 & - & 6,9 & 6,8 \\
\hline Total & 100,0 & 100,0 & 100,0 & 100,0 & 100,0 & 100,0 \\
\hline Effectifs & 22176 & 6676920 & 6699096 & 13793 & 2130402 & 2144195 \\
\hline
\end{tabular}

Source : Données de l'enquête auprès des ménages, EADE-RDC 2012

On trouve plus d'enfants de 3-5 ans en dehors de l'école en milieu rural (tableau 15). Par contre, il y a plus de déscolarisés en milieu urbain qu'en milieu rural. Pour les enfants n'ayant jamais fréquenté, c'est plus en milieu rural qu'ils se retrouvent. La répartition des enfants en dehors de l'école de 3-5 ans par province reste presque calquée sur celle de la population. On relève cependant qu'en termes de déscolarisés et de ceux n'ayant jamais fréquenté l'école, la répartition est tout autre et serait plus liée à l'accès aux structures du pré-primaire. Ce qui implique que la déscolarisation soit un phénomène urbain. Elle est très nette pour les enfants de 5 ans à Kinshasa (tableau 15).

\subsubsection{Caractéristiques du chef de ménage}

Le tableau 16 présente la répartition des enfants de 3-5 ans en dehors de l'école selon certaines caractéristiques du chef de ménage. II en ressort que trois enfants sur cinq, soit 59,1\% vivent dans des ménages dirigés par des hommes et deux enfants sur cinq, soit $40,9 \%$ sont dans des ménages dirigés par une femme. Pour les déscolarisés et les jamais scolarisés, ils sont toujours proportionnellement plus nombreux dans les ménages dirigés par un homme. En termes absolus, l'essentiel des enfants en dehors de l'école de 35 ans n'ont jamais été à l'école (6 677 920) et résident également avec des chefs de ménage qui eux aussi n'ont pas été à l'école $(69,5 \%)$. 
Tableau 16 : Répartition (en \%) des enfants d'âges du pré-primaire en dehors de l'école par statut scolaire selon les caractéristiques du chef de ménage

\begin{tabular}{|c|c|c|c|c|c|c|}
\hline \multirow[b]{2}{*}{ Caractéristiques } & \multicolumn{3}{|c|}{ Enfants de $3-5$ ans } & \multicolumn{3}{|c|}{ Enfants de 5 ans } \\
\hline & Déscolarisé & $\begin{array}{l}\text { Jamais } \\
\text { fréquenté }\end{array}$ & Ens. & Déscolarisé & $\begin{array}{l}\text { Jamais } \\
\text { fréquenté }\end{array}$ & Ens. \\
\hline \multicolumn{7}{|c|}{ Sexe du chef de ménage } \\
\hline Hommes & 54,2 & 59,1 & 59,1 & 60,5 & 59,2 & 59,3 \\
\hline Femmes & 45,8 & 40,9 & 40,9 & 39,5 & 40,8 & 40,7 \\
\hline Total & 100,0 & 100,0 & 100,0 & 100,0 & 100,0 & 100,0 \\
\hline \multicolumn{7}{|c|}{ Niveau d'instruction du CM } \\
\hline Aucun & 73,1 & 69,4 & 69,5 & 70,0 & 71,4 & 71,4 \\
\hline Primaire & 10,2 & 8,6 & 8,6 & 16,4 & 8,5 & 8,6 \\
\hline Secondaire+ & 16,7 & 22,0 & 22,0 & 13,6 & 20,1 & 20,0 \\
\hline Total & 100,0 & 100,0 & 100,0 & 100,0 & 100,0 & 100,0 \\
\hline Effectifs & 22176 & 6676920 & 6699096 & 13793 & 2130402 & 2144195 \\
\hline
\end{tabular}

Source : Données de l'enquête auprès des ménages, EADE-RDC 2012

\subsubsection{Revenu du ménage}

Les enfants en dehors de l'école d'âges du pré-primaire sont issus pour plus de la moitié des ménages à faible revenu mensuel. Sur les 6699099 enfants de 3-5 ans, il y a 56,1\% qui sont issus des ménages à revenu mensuel inférieur à 50 USD. On trouve seulement 5,8\% des enfants en dehors de l'école d'âges du pré-primaire qui sont issus des ménages à revenu mensuel supérieur à 200 USD (tableau 17). Parmi les enfants d'âges du pré-primaire qui sont déscolarisés il y a une répartition presqu'uniforme de ceux-ci dans les différentes classes de revenu mensuel des ménages. Chez les enfants de 3-5 ans jamais scolarisés, plus de la moitié sont dans des ménages avec un revenu mensuel de moins de 50 USD. Il en est de même des enfants en dehors de l'école de 5 ans.

Tableau 17 : Répartition (en \%) des enfants d'âges du pré-primaire en dehors de l'école par statut scolaire selon le revenu mensuel du ménage

\begin{tabular}{|c|c|c|c|c|c|c|}
\hline \multirow{2}{*}{$\begin{array}{l}\text { Revenu mensuel du } \\
\text { ménage }\end{array}$} & \multicolumn{3}{|c|}{ Enfants de $3-5$ ans } & \multicolumn{3}{|c|}{ Enfants de 5 ans } \\
\hline & Déscolarisé & $\begin{array}{l}\text { Jamais } \\
\text { fréquenté }\end{array}$ & Ens. & Déscolarisé & $\begin{array}{l}\text { Jamais } \\
\text { fréquenté }\end{array}$ & Ens. \\
\hline Moins de 50 USD & 24,4 & 56,2 & 56,1 & 16,4 & 58,4 & 58,1 \\
\hline 50 à 100 USD & 22,6 & 27,3 & 27,3 & 22,6 & 27,3 & 27,3 \\
\hline 101 à 200 USD & 28,7 & 10,7 & 10,8 & 30,9 & 10,4 & 10,5 \\
\hline Plus de 200 USD & 24,3 & 5,8 & 5,8 & 30,1 & 4,0 & 4,1 \\
\hline Total & 100,0 & 100,0 & 100,0 & 100,0 & 100,0 & 100,0 \\
\hline Effectifs & 22176 & 6676920 & 6699096 & 13793 & 2130402 & 2144195 \\
\hline
\end{tabular}

Source : Données de l'enquête auprès des ménages, EADE-RDC 2012 


\subsection{Profil des EADE d'âges du primaire et du secondaire (Dimensions 2 et 3)}

Les enfants et adolescents en dehors de l'école d'âges du primaire sont ceux du groupe d'âges 6-11 ans (dimension 2) et ceux d'âges du secondaire (classes inférieures du secondaire premier cycle) sont du groupe d'âges 12-13 ans (dimension 3).

\subsubsection{Sexe de l'enfant et répartition spatiale des EADE de 6-11 ans et de 12-13 ans}

\section{a) Enfants d'âges du primaire en dehors de l'école}

L'enquête EADE de 2012 a estimé les enfants en dehors de l'école de la dimension 2 (6-11 ans) à 3509253 individus. Parmi eux, il y a presqu'autant de filles en dehors de l'école que de garçons (tableau 18). Les déscolarisés y représentent $15,8 \%$ des EADE (555 511).

Tableau 18 : Répartition (en \%) des enfants et adolescents en dehors de l'école par statut scolaire et âges de scolarisation (6-11 ans et 12-13 ans) selon le sexe, l'âge, le milieu de résidence et la province

\begin{tabular}{|c|c|c|c|c|c|c|}
\hline \multirow[b]{2}{*}{ Caractéristiques } & \multicolumn{3}{|c|}{ Dimension 2 (6-11 ans) } & \multicolumn{3}{|c|}{ Dimension 3 (12-13 ans) } \\
\hline & Déscolarisé & $\begin{array}{c}\text { Jamais } \\
\text { fréquenté }\end{array}$ & Total & Déscolarisé & $\begin{array}{c}\text { Jamais } \\
\text { fréquenté }\end{array}$ & Total \\
\hline \multicolumn{7}{|l|}{ Sexe } \\
\hline Garçons & 49,5 & 49,9 & 49,9 & 43,5 & 35,1 & 39,6 \\
\hline Filles & 50,5 & 50,1 & 50,1 & 56,5 & 64,9 & 60,4 \\
\hline Total & 100,0 & 100,0 & 100,0 & 100,0 & 100,0 & 100,0 \\
\hline \multicolumn{7}{|l|}{ Milieu } \\
\hline Urbain & 44,2 & 17,4 & 21,7 & 31,1 & 14,0 & 23,2 \\
\hline Rural & 55,8 & 82,6 & 78,3 & 68,9 & 86,0 & 76,8 \\
\hline Total & 100,0 & 100,0 & 100,0 & 100,0 & 100,0 & 100,0 \\
\hline \multicolumn{7}{|l|}{ Provinces } \\
\hline Kinshasa & 20,7 & 3,3 & 6,1 & 11,6 & 1,0 & 6,7 \\
\hline Bas-Congo & 2,4 & 4,9 & 4,5 & 6,6 & 4,4 & 5,6 \\
\hline Bandundu & 3,8 & 8,6 & 7,8 & 2,5 & 4,9 & 3,6 \\
\hline Equateur & 5,7 & 10,8 & 10,0 & 6,5 & 13,0 & 9,5 \\
\hline Orientale & 17,1 & 12,8 & 13,5 & 18,3 & 14,3 & 16,4 \\
\hline Nord Kivu & 10,1 & 13,6 & 13,1 & 15,1 & 18,8 & 16,8 \\
\hline Maniema & 1,0 & 2,5 & 2,2 & 1,2 & 2,0 & 1,5 \\
\hline sud Kivu & 6,7 & 6,7 & 6,7 & 7,0 & 6,9 & 7,0 \\
\hline Katanga & 18,7 & 19,4 & 19,3 & 18,1 & 23,6 & 20,7 \\
\hline Kasaï oriental & 9,0 & 10,1 & 9,9 & 7,2 & 8,3 & 7,7 \\
\hline Kasaï occidental & 4,8 & 7,4 & 6,9 & 5,9 & 2,8 & 4,5 \\
\hline Total & 100,0 & 100,0 & 100,0 & 100,0 & 100,0 & 100,0 \\
\hline Effectifs & 555511 & 2953742 & 3509253 & 275901 & 237267 & 513168 \\
\hline
\end{tabular}

Source : Données de l'enquête auprès des ménages, EADE-RDC 2012

En milieu rural, il y a proportionnellement plus de déscolarisés d'âges du primaire (55,8 \%) qu'en milieu urbain $(44,2 \%)$. Cette différence est encore plus accentuée avec les enfants qui n'ont jamais fréquenté l'école (tableau 18). En termes de distribution par province, la province du Katanga à elle seule regroupe près d'un EADE d'âges du primaire sur cinq (19,4\%). Elle est suivie du Nord Kivu (13,6 \%) et de la province orientale $(12,8 \%)$. Mais en termes de déscolarisés, ce sont Kinshasa $(20,7 \%)$, le Katanga $(18,7 \%)$ et la Province Orientale $(17,1 \%)$ qui ont les proportions les plus élevées. 


\section{b) Adolescents d'âge du secondaire premier cycle qui sont en dehors de l'école}

On dénombre 513168 enfants d'âges du premier cycle du secondaire (12-13 ans) qui sont en dehors de l'école, dont 237267 n'ayant jamais fréquenté l'école et 275901 ayant abandonné (tableau 18). La proportion des déscolarisés est nettement plus importante que ce qu'on observe au niveau du primaire (plus on avance dans le système plus les abandons sont importants).

II y a proportionnellement 2 fois plus de déscolarisés (31,1\%) que ceux n'ayant jamais fréquenté en milieu urbain (14,0\%). La distribution par province des EADE d'âges du premier cycle du secondaire montre encore que la province du Katanga est en tête $(20,7 \%)$, suivie de la province du Nord Kivu (16,8\%) et de la province orientale $(16,4 \%)$.

\subsubsection{Caractéristiques du chef de ménage}

La répartition des enfants d'âges du primaire qui sont en dehors de l'école selon le niveau d'instruction du chef de ménage montre que dans l'ensemble, près de 6 EADE sur 10 sont dans des ménages où le chef de ménage n'a pas été à l'école, alors que seulement $16,2 \%$ des EADE vivent dans des ménages où le chef de ménage est de niveau d'instruction primaire. II ressort du tableau 18 que six enfants sur dix, soit 60,2\% vivent dans des ménages dirigés par des hommes et quatre enfants sur dix, soit $39,8 \%$ sont dans des ménages dirigés par une femme (tableau 19).

Tableau 19 : Répartition (en \%) des enfants et adolescents en dehors de l'école par statut scolaire et âges de scolarisation (6-11 ans et 12-13 ans) selon certaines caractéristiques

\begin{tabular}{|c|c|c|c|c|c|c|}
\hline \multirow[t]{2}{*}{ Caractéristiques } & \multicolumn{3}{|c|}{ Dimension 2 (6-11 ans) } & \multicolumn{3}{|c|}{ Dimension 3 (12-13 ans) } \\
\hline & Déscolarisé & $\begin{array}{l}\text { Jamais } \\
\text { fréquenté }\end{array}$ & Total & Déscolarisé & $\begin{array}{l}\text { Jamais } \\
\text { fréquenté }\end{array}$ & Total \\
\hline \multicolumn{7}{|c|}{ Sexe du chef de ménage } \\
\hline Hommes & 58,7 & 60,4 & 60,2 & 65,1 & 69,4 & 67,1 \\
\hline Femmes & 41,3 & 39,6 & 39,8 & 34,9 & 30,6 & 32,9 \\
\hline Total & 100,0 & 100,0 & 100,0 & 100,0 & 100,0 & 100,0 \\
\hline \multicolumn{7}{|c|}{ Niveau d'instruction du CM } \\
\hline Aucun & 60,0 & 66,3 & 65,3 & 43,7 & 49,8 & 46,5 \\
\hline Primaire & 18,2 & 15,8 & 16,2 & 26,2 & 27,0 & 26,6 \\
\hline Secondaire+ & 21,9 & 17,9 & 18,6 & 30,1 & 23,2 & 26,9 \\
\hline Total & 100,0 & 100,0 & 100,0 & 100,0 & 100,0 & 100,0 \\
\hline Effectifs & 555511 & 2953742 & 3509253 & 275901 & 237267 & 513168 \\
\hline
\end{tabular}

Source : Données de l'enquête auprès des ménages, EADE-RDC 2012

Pour les enfants d'âges du secondaire les mêmes tendances sont observées, mais avec des niveaux différents. En effet, moins de la moitié $(46,5 \%)$ des EADE de $12-13$ ans sont dans des ménages où le chef n'a pas été à l'école.

\subsubsection{Revenu du ménage}

Les EADE dans l'ensemble se retrouvent davantage dans les ménages les plus démunis (tableau 20). En effet, $64,3 \%$ des enfants et adolescents d'âges du primaire en dehors de l'école et autant pour les enfants et adolescents d'âges du secondaire en dehors de l'école $(64,9 \%)$ sont issus des ménages les plus pauvres (moins de 50 \$ par mois), alors que respectivement 2,9\% et 5,2\% de ces enfants viennent des ménages nantis (plus de 200 USD par mois). 
Tableau 20 : Répartition (en \%) des enfants et adolescents en dehors de l'école par statut scolaire et âges de scolarisation (6-11 ans et 12-13 ans) selon le revenu mensuel du ménage.

\begin{tabular}{|c|c|c|c|c|c|c|}
\hline \multirow[b]{2}{*}{ Revenu mensuel du ménage } & \multicolumn{3}{|c|}{ Dimension 2 (6-11 ans) } & \multicolumn{3}{|c|}{ Dimension 3 (12-13 ans) } \\
\hline & Déscolarisé & $\begin{array}{c}\text { Jamais } \\
\text { fréquenté }\end{array}$ & Total & Déscolarisé & $\begin{array}{c}\text { Jamais } \\
\text { fréquenté }\end{array}$ & Total \\
\hline Moins de 50 USD & 51,2 & 66,8 & 64,3 & 57,9 & 73,0 & 64,9 \\
\hline 50 à 100 USD & 27,2 & 24,2 & 24,7 & 21,7 & 16,7 & 19,4 \\
\hline 101 à 200 USD & 15,0 & 6,8 & 8,1 & 15,0 & 5,3 & 10,5 \\
\hline Plus de 200 USD & 6,6 & 2,2 & 2,9 & 5,4 & 4,9 & 5,2 \\
\hline Total & 100,0 & 100,0 & 100,0 & 100,0 & 100,0 & 100,0 \\
\hline Effectifs & 555511 & 2953742 & 3509253 & 275901 & 237267 & 513168 \\
\hline
\end{tabular}

Source : Données de l'enquête auprès des ménages, EADE-RDC 2012

\subsubsection{Typologie des enfants et adolescents en dehors de l'école}

En référence au cadre conceptuel et méthodologique (CMF), une analyse de la typologie des EADE a été faite. Elle a permis de préciser pour ce qui est des EADE, ceux qui ont abandonné, ceux qui entreront plus tard à l'école avant 17 ans et ceux qui n'entreront jamais. II ressort que pour les enfants d'âges du primaire en dehors de l'école (26,7\% des 6-11 ans), ceux qui entreront à l'école d'ici leur $17^{\text {ème }}$ anniversaire représentent $74,2 \%$, ceux qui vont abandonner représentent $15,8 \%$ et ceux qui n'entreront jamais à l'école représentent $10,0 \%$ (graphique 8). Les effectifs correspondant à chaque catégorie sont donnés au tableau A7 en annexe.

Graphique 8 : Typologie des enfants et adolescents en dehors de l'école

Enfants d'âges du primaire secondaire

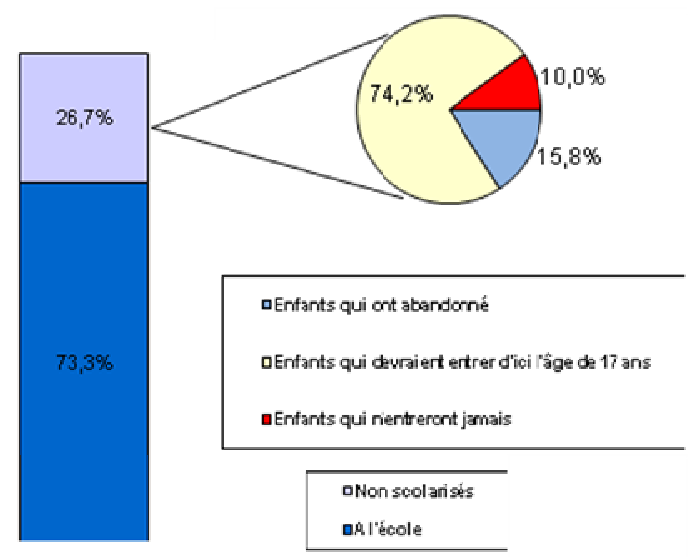

Enfants d'âges du $1^{\mathrm{er}}$ cycle du

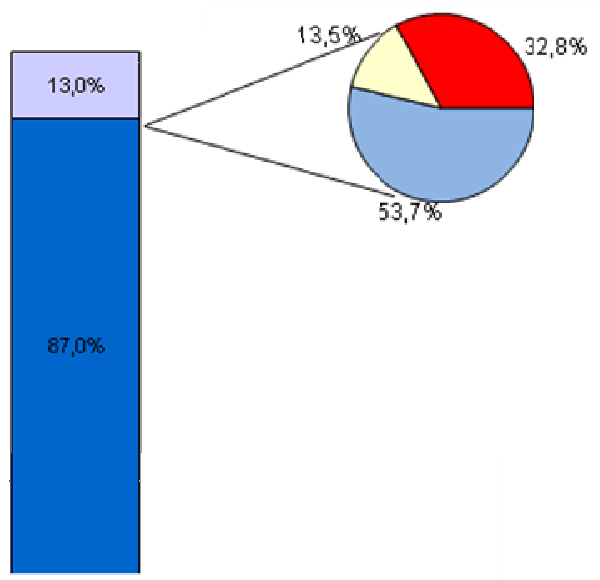

Source : Données de l'enquête auprès des ménages, EADE-RDC 2012

Pour les enfants d'âges du $1^{\text {er }}$ cycle du secondaire en dehors de l'école (13,0 \% des $12-13$ ans), ceux qui abandonnent dépassent plus de la moitié des $\operatorname{EADE}(53,7 \%)$. Très peu $(13,4 \%)$ entreront avant 17 ans et le tiers n'entrera jamais à l'école (graphique 8).

\subsection{Profil des enfants et adolescents à risque d'abandonner (Dimension 4 et 5)}

Les dimensions 4 et 5 traitent principalement des enfants qui courent le plus grand risque d'abandonner. II existe plusieurs indicateurs permettant de caractériser la population des enfants à risque. Par exemple, la scolarisation au préscolaire est apparue dans la littérature comme pouvant réduire la probabilité de déperdition scolaire plus tard dans le parcours scolaire (Hammond et al., 2007). Ainsi, le taux de nouveaux inscrits au niveau primaire sans aucune expérience d'éduction préscolaire (Early Childhood Care and Education : ECCE) constitue un indicateur du risque d'abandon. Le retard scolaire, le travail des enfants, ainsi que certaines 
caractéristiques familiales sont des facteurs de risque d'abandon (Potvin et Al., 1999 ; HTSPE - EUROPE, 2012; Diagne, 2006).

L'Institut de Statistique de l'UNESCO (ISU) dans la note méthodologique de juin 2011 sur l'analyse des dimensions 4 et 5 arrive à la conclusion que la meilleure approche pour mesurer l'effectif des élèves du primaire et du premier cycle du secondaire à risque d'abandonner est de recourir aux taux d'abandons de ceux qui ont déjà quitté le système scolaire. On peut également présenter les statistiques sur le retard scolaire, mais dans ce cas, il convient de s'en tenir aux enfants ayant deux années de retard scolaire par rapport à leur classe, pour éviter de surestimer le nombre d'enfants à risque d'abandonner.

\subsubsection{Estimation du risque d'abandonner à partir des taux d'abandon déjà réalisés}

En recourant aux taux d'abandons récents observés (entre deux années scolaires successives) comme étant la meilleure mesure du pourcentage des enfants à risque d'abandonner, on voit bien (tableau 21) que les niveaux obtenus sont très différents de la proportion des enfants ayant au moins deux années de retard scolaire. Au niveau du primaire, le taux d'abandon est de 2,5\%. Le taux d'abandon pour le premier cycle du secondaire n'est pas très différent $(2,4 \%)$. Ces taux sont également très faibles car portant uniquement sur l'abandon entre deux années scolaires. Ils ne donnent donc pas une bonne mesure du risque d'abandonner sur l'ensemble du cycle pour un enfant scolarisé. 
Tableau 21 : Taux d'abandon (en $\%$ ) par classe du primaire et $\mathrm{du}$ secondaire $1^{\text {er }}$ cycle des enfants et adolescents

\begin{tabular}{|c|c|c|c|c|c|c|c|c|c|c|}
\hline \multirow{2}{*}{ Caractéristiques } & \multicolumn{7}{|c|}{ Primaire } & \multicolumn{3}{|c|}{ Secondaire } \\
\hline & 1 & 2 & 3 & 4 & 5 & 6 & Ens. & 7 & 8 & Ens. \\
\hline \multicolumn{11}{|l|}{ Sexe } \\
\hline Garçons & 2,9 & 2,7 & 2,8 & 2,9 & 2,4 & 2,3 & 2,6 & 2,2 & 2,4 & 2,3 \\
\hline Filles & 3,5 & 2,5 & 2,9 & 2,1 & 1,5 & 1,7 & 2,4 & 2,1 & 2,9 & 2,5 \\
\hline \multicolumn{11}{|l|}{ Milieu } \\
\hline Urbain & 4,3 & 3,9 & 4,5 & 2,9 & 2,9 & 2,0 & 3,4 & 2,4 & 2,4 & 2,4 \\
\hline Rural & 2,8 & 2,1 & 2,2 & 2,3 & 1,5 & 2,0 & 2,2 & 2,0 & 2,8 & 2,4 \\
\hline \multicolumn{11}{|l|}{ Provinces } \\
\hline Kinshasa & 4,3 & 4,5 & 4,7 & 4,3 & 3,2 & 2,1 & 3,8 & 1,3 & 2,7 & 2,1 \\
\hline Bas-Congo & 3,5 & 1,8 & 1,3 & 2,8 & 1,6 & 1,7 & 2,1 & 2,0 & 1,6 & 1,8 \\
\hline Bandundu & 1,3 & 0,9 & 0,4 & 0,9 & 0,8 & 0,1 & 0,7 & 1,3 & 3,6 & 2,5 \\
\hline Equateur & 4,3 & 1,5 & 2,9 & 0,3 & 1,1 & 2,8 & 2,2 & 3,0 & 5,0 & 4,0 \\
\hline Orientale & 1,9 & 2,4 & 2,6 & 4,1 & 1,9 & 1,1 & 2,3 & 1,9 & 0,5 & 1,2 \\
\hline Nord Kivu & 4,5 & 3,7 & 4,1 & 4,8 & 3,0 & 2,9 & 3,8 & 3,2 & 5,8 & 4,6 \\
\hline Maniema & 2,8 & 0,5 & 1,8 & 0,8 & 1,0 & 2,1 & 1,6 & 1,1 & 3,0 & 2,2 \\
\hline sud Kivu & 3,3 & 1,6 & 1,5 & 3,1 & 1,6 & 3,2 & 2,4 & 3,5 & 1,3 & 2,3 \\
\hline Katanga & 5,1 & 4,0 & 3,9 & 2,6 & 2,5 & 2,9 & 3,5 & 2,8 & 2,3 & 2,5 \\
\hline Kasaï oriental & 1,0 & 3,3 & 4,2 & 1,8 & 2,2 & 1,7 & 2,3 & 0,7 & 0,9 & 0,8 \\
\hline Kasaï occidental & 3,5 & 1,6 & 1,6 & 0,3 & 1,3 & 2,3 & 1,8 & 4,2 & 2,5 & 3,3 \\
\hline \multicolumn{11}{|c|}{ Classes de revenu mensuel } \\
\hline Moins de 50 dollars & 3,4 & 2,5 & 2,9 & 2,6 & 1,3 & 2,1 & 2,5 & 2,7 & 2,6 & 2,7 \\
\hline $50-100$ dollars & 2,8 & 2,1 & 2,4 & 2,2 & 2,6 & 1,7 & 2,3 & 1,6 & 2,9 & 2,3 \\
\hline 101-200 dollars & 4,3 & 4,4 & 3,1 & 3,4 & 2,3 & 1,9 & 3,2 & 2,2 & 2,5 & 2,4 \\
\hline Plus de 200 dollars & 1,5 & 2,9 & 3,7 & 1,2 & 3,1 & 2,5 & 2,5 & 1,1 & 2,3 & 1,8 \\
\hline \multicolumn{11}{|l|}{ Sexe CM } \\
\hline Homme & 3,2 & 2,4 & 2,4 & 2,5 & 1,6 & 2,4 & 2,4 & 2,2 & 2,5 & 2,4 \\
\hline Femmes & 3,2 & 2,8 & 3,6 & 2,4 & 2,4 & 1,4 & 2,7 & 2,0 & 2,8 & 2,4 \\
\hline \multicolumn{11}{|c|}{ Niveau d'instruction CM } \\
\hline Aucun & 3,6 & 2,5 & 3,1 & 2,6 & 2,1 & 1,7 & 2,6 & 2,0 & 2,2 & 2,1 \\
\hline Primaire & 3,1 & 3,6 & 3,3 & 2,8 & 2,2 & 2,3 & 2,9 & 0,0 & 0,0 & 0,0 \\
\hline Secondaire+ & 2,6 & 2,0 & 2,2 & 2,1 & 1,5 & 2,3 & 2,1 & 2,3 & 3,1 & 2,7 \\
\hline Ensemble RDC & 3,2 & 2,6 & 2,8 & 2,5 & 1,9 & 2,0 & 2,5 & 2,1 & 2,7 & 2,4 \\
\hline
\end{tabular}

Source : Données de l'enquête auprès des ménages, EADE-RDC 2012

La mesure du risque d'abandonner après l'entrée à l'école comme mesuré dans l'EADE permet de mieux apprécier le risque d'abandonner sur l'ensemble du cycle. En effet, un des apports de l'enquête EADE-RDC 2012 est l'analyse du parcours scolaire des enfants et adolescents, notamment en termes d'entrée à l'école, de redoublement, d'interruption, d'absence scolaire et d'abandon. L'un des défis importants de cette collecte de données sur les parcours scolaires est celui de la bonne datation des événements collectés en vue d'une description de leur occurrence dans le temps. 


\subsubsection{Risque d'abandonner au cours du cycle}

L'examen des probabilités cumulées d'abandonner après l'entrée à l'école montre que durant tout le cursus scolaire, les filles présentent des risques plus élevés d'abandonner que les garçons (graphique 9). Cette différence entre filles et garçons se renforce tout le long du cursus scolaire : ainsi, 6 années après l'entrée à l'école (ce qui correspond à la fin du primaire pour un parcours sans redoublement), 16,6 \% des filles ont déjà abandonné contre $12,1 \%$ des garçons. Cette probabilité cumulée d'abandonner après 6 années pourrait être aussi une mesure des taux d'abandon pour les élèves de la dimension 4. A 12 ans après l'entrée à l'école (ce qui correspond à la fin du secondaire pour un parcours sans faute), 38,8 \% des filles ont déjà quitté le système scolaire contre 30,7 \% pour les garçons (tableau A18 en annexe).

Graphique 9 : Probabilités cumulées (en \%) d'abandonner par sexe et par milieu de résidence

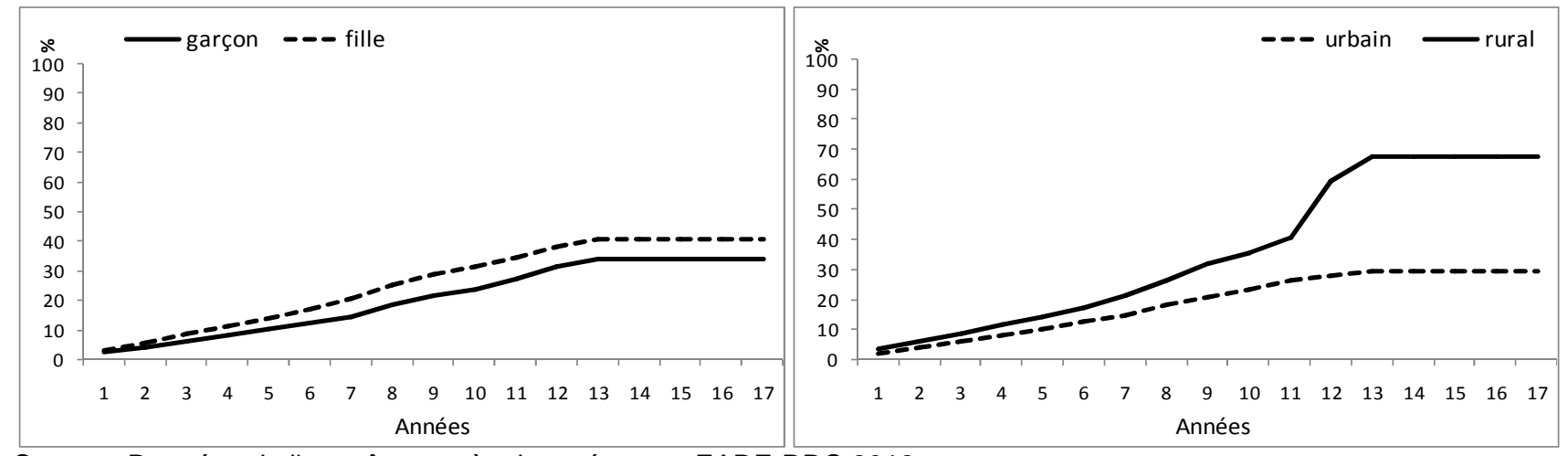

Source : Données de l'enquête auprès des ménages, EADE-RDC 2012

L'écart entre le milieu urbain et le milieu rural en termes de risque d'abandon scolaire est encore plus important : 12 ans après l'entrée à l'école $27,4 \%$ des enfants en milieu urbain ont déjà abandonné l'école, alors que cette proportion est de $45,6 \%$ en milieu rural (un écart absolu de 18 points). Il apparaît également des variations provinciales énormes en ce qui concerne les probabilités d'abandonner. Le Bandundu se caractérise par des risques d'abandons très faibles au cours du cycle comparativement aux autres provinces, résultat qui mérité un examen plus approfondi des données et du contexte, car la différence avec les autres provinces est énorme. Les pourcentages les plus élevés se situent au Nord Kivu (39,8\%), au Kasaï Occidental (38,5\%), au Sud Kivu (36,9\%), dans la Province Orientale (35,9\%) (tableau A19 en annexe).

\subsubsection{Age d'entrée à l'école}

L'entrée tardive à l'école est aussi un élément qui pourrait entraîner un retard scolaire. Le graphique 10 donne les probabilités cumulées d'entrée à l'école respectivement par sexe, par milieu de résidence et par province. On note qu'il n'y a pas différence significative entre garçons et filles en termes d'entrée à l'école : en effet, à 6 ans, un garçon sur cinq $(20,9)$ et une fille sur cinq $(20,3)$ sont déjà entrés l'école. A 9 ans, $73,1 \%$ des garçons et $69,8 \%$ des filles sont déjà entrés à l'école (tableau A20 en annexe).

S'il n'y a pas de différence énorme entre garçons et filles en termes de chances d'entrée à l'école, en ce qui concerne le milieu de résidence par contre, la différence entre les courbes d'entrée à l'école est statistiquement significative : A 6 ans, plus d'un enfant sur trois en ville est déjà entré à l'école $(34,5 \%)$ alors qu'à la campagne, seulement un peu plus d'un enfant sur dix (13,5\%) est déjà à l'école à cet âge. De même, à 9 ans, on note que les enfants du milieu urbain sont à $79,1 \%$ déjà entrés à l'école, proportion qui se situe à $67,6 \%$ en milieu rural (tableau A20 en annexe).

II existe également une variation entre provinces en termes de probabilités d'entrée à l'école. On note tout d'abord un phénomène quasi-général d'entrée tardive des enfants à l'école dans toutes les provinces : dans aucune des onze provinces, la proportion d'enfants qui sont déjà à l'école à 6 ans n'atteint $50 \%$. A 7 ans, 65,0 $\%$ des enfants sont déjà entrés à l'école à Kinshasa et dans les autres provinces, ce n'est qu'à 8 ans que plus de la moitié des enfants sont déjà à l'école ; le Bas-Congo et le Sud Kivu venant après Kinshasa (73,9 \%) avec respectivement $63,3 \%$ et $61,6 \%$ (tableau A21 en annexe). 
Graphique 10 : Probabilités cumulées (en \%) d'entrée à l'école par sexe et par milieu de résidence

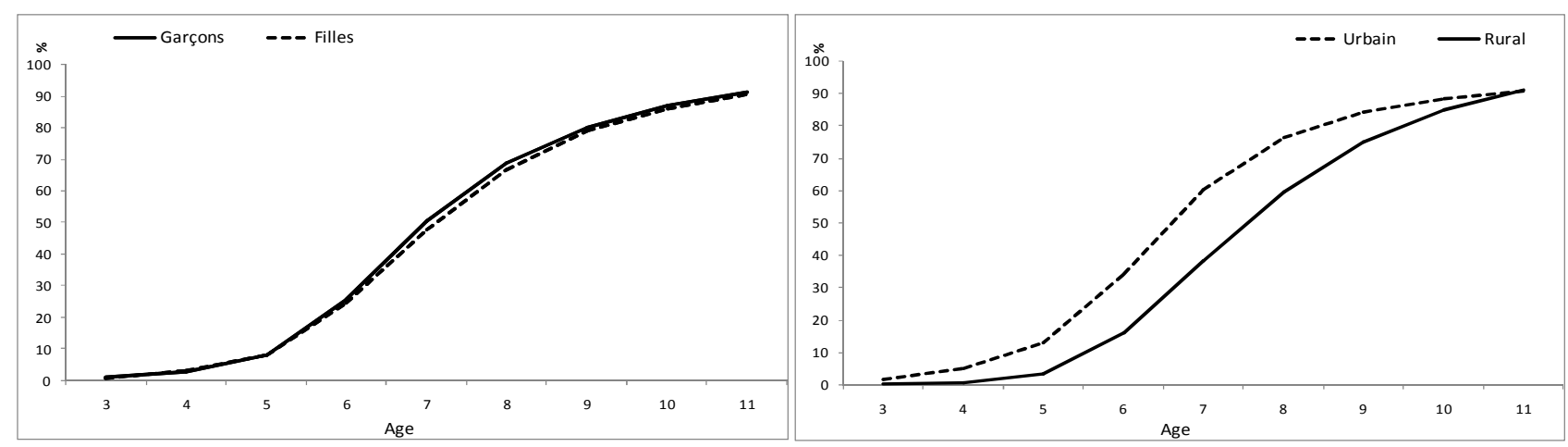

Source : Données de l'enquête auprès des ménages, EADE-RDC 2012

\subsubsection{Retard scolaire}

A partir des informations sur l'âge, le niveau et la classe fréquentée, on peut déterminer la situation actuelle des enfants en termes de retard (ou de précocité) par rapport à la classe fréquentée.

a) Le primaire

II ressort du tableau 22 que $77,2 \%$ des élèves du primaire sont en retard d'au moins un an par rapport à leur classe. Considérer une année de retard scolaire comme un facteur de risque d'abandon surestime énormément la réalité car il est difficile de penser que plus de 3 élèves du primaire sur 4 abandonneront probablement. Même en considérant deux années de retard scolaire, on a plus de la moitié des élèves du primaire $(55,3 \%)$ qui seraient à risque d'abandonner. II n'y a pas de différence majeure entre filles et garçons, les premières étant $56,0 \%$ à connaître au moins deux années de retard et les derniers 54,8\%. Par contre, entre milieux, les écarts sont importants $(63,8 \%$ des élèves du primaire ont au moins deux années de retard en milieu rural et $37,0 \%$ en milieu urbain).

Tableau 22 : Proportions (en \%) des enfants et adolescents scolarisés au primaire selon le retard scolaire par sexe et par milieu de résidence

\begin{tabular}{|c|c|c|c|c|c|}
\hline Situation scolaire & Garçons & Filles & Urbain & Rural & Ensemble \\
\hline Précoce & 6,2 & 6,4 & 10,5 & 4,3 & 6,3 \\
\hline Normal & 16,7 & 16,2 & 26,3 & 12,0 & 16,5 \\
\hline Tardif 1 an & 77,2 & 77,4 & 63,3 & 83,7 & 77,2 \\
\hline Tardif 2 ans & 54,8 & 56,0 & 37,0 & 63,8 & 55,3 \\
\hline Tardif 3 ans + & 37,7 & 37,8 & 22,0 & 45,0 & 37,7 \\
\hline Effectifs & 8126073 & 7100617 & 4794165 & 10432523 & 15226690 \\
\hline
\end{tabular}

Source : Données de l'enquête auprès des ménages, EADE-RDC 2012

\section{b) Le secondaire}

Les schémas observés au niveau du primaire se maintiennent au niveau du premier cycle du secondaire, sauf que les proportions d'élèves en retard scolaire sont encore plus importantes. II ressort (tableau 23) que 70,4\% des élèves du premier cycle du secondaire sont en retard scolaire d'au moins deux années. Cette proportion est plus forte chez les garçons que chez les filles (respectivement $71,8 \%$ et $68,3 \%$ ) et plus élevée en milieu rural $(82,4 \%)$ qu'en milieu urbain $(57,1 \%)$. 
Tableau 23 : Proportions (en \%) des enfants et adolescents scolarisés au secondaire $1^{\text {er }}$ cycle selon le retard scolaire par sexe et par milieu de résidence

\begin{tabular}{|c|c|c|c|c|c|}
\hline Situation scolaire & Garçons & Filles & Urbain & Rural & Ensemble \\
\hline Précoce & 4,0 & 3,5 & 5,6 & 2,2 & 3,8 \\
\hline Normal & 8,8 & 10,5 & 15,8 & 3,8 & 9,5 \\
\hline Tardif 1 an & 87,2 & 85,9 & 78,7 & 93,9 & 86,7 \\
\hline Tardif 2 ans & 71,8 & 68,3 & 57,1 & 82,4 & 70,4 \\
\hline Tardif 3 ans + & 54,2 & 50,7 & 37,5 & 66,6 & 52,8 \\
\hline Effectifs & 1336267 & 985808 & 1043362 & 1278719 & 2322075 \\
\hline
\end{tabular}

Source : Données de l'enquête auprès des ménages, EADE-RDC 2012

\subsubsection{Phénomène des absences au cours de l'année scolaire 2010-2011}

\section{a) Fréquences}

L'absence scolaire peut être un facteur d'échec car si l'absence est assez longue et répétitive, l'élève ne bénéficiera pas d'une formation complète. II est donc intéressant d'analyser les absences pour apprécier leur incidence dans l'échec des enfants. Au cours de l'année scolaire 2010-2011, on observe 3,4 absents pour 100 élèves. Ce taux d'absence est légèrement moins élevé chez les filles (3,3 absentes pour 100 filles contre 3,5 chez les garçons).

Tableau 24 : Proportions d'élèves absents (en \%) par province, sexe et milieu de résidence, année scolaire 2010-2011

\begin{tabular}{lccccc}
\hline Province & Garçon & Fille & Urbain & Rural & Ensemble \\
\hline Kinshasa & 5,4 & 3,1 & 4,2 & & 4,2 \\
Bas-Congo & 0,8 & 2,8 & 1,6 & 1,8 & 1,7 \\
Bandundu & 2,0 & 0,7 & 2,5 & 1,1 & 1,4 \\
Equateur & 0,8 & 2,7 & 1,0 & 1,9 & 1,8 \\
Province orientale & 4,9 & 4,3 & 4,4 & 4,6 & 4,6 \\
Nord-Kivu & 2,5 & 2,6 & 1,5 & 3,0 & 2,6 \\
Maniema & 2,2 & 2,2 & 2,8 & 2,0 & 2,2 \\
Sud-Kivu & 4,0 & 4,0 & 1,8 & 4,6 & 4,0 \\
Katanga & 1,8 & 1,7 & 2,2 & 1,4 & 1,7 \\
Kasaï oriental & 9,7 & 10,9 & 5,5 & 14,9 & 10,3 \\
Kasaï occidental & 4,0 & 4,3 & 3,5 & 4,2 & 4,1 \\
\hline Ensemble & 3,5 & 3,4 & 3,4 & 3,4 & 3,4 \\
\hline Source : Donné
\end{tabular}

Source : Données de l'enquête auprès des ménages, EADE-RDC 2012

Ces absences paraissent faibles contrairement à l'idée qu'on se faisait du phénomène. Mais cela peut être dû à la définition de l'absence adoptée par l'enquête EADE. En effet, la durée d'absence minimale prise en compte dans le questionnaire est de 4 semaines. Or les absences scolaires les plus fréquentes sont de très courtes durées ne dépassant pas généralement une à deux semaines.

Entre le milieu urbain et le milieu rural globalement à l'échèle du pays il n'y a pratiquement pas de différence. Mais un examen par province fait ressortir des écarts importants entre milieu rural et urbain. Ainsi au Kasaï oriental en milieu rural la proportion des absents est de $14,9 \%$ contre $5,5 \%$ en milieu urbain, au Sud-Kivu (4,6\% contre 1,8\%), au Nord-Kivu (3,0\% contre 1,5\%), à l'Equateur (1,9\% contre 1,0\%). Dans d'autres provinces telles qu'au Katanga, dans le Bandundu, c'est en ville que les proportions d'absents sont les plus élevées (tableau 24). Les cinq provinces où les proportions d'absents sont les plus élevées sont par ordre d'importance, le Kasaï oriental, la Province orientale, Kinshasa, le Kasaï Occidental et le Sud-Kivu. 


\section{b) Classes fréquentées et absences}

Au niveau du pré-primaire des absences sont observées en première et troisième année. Au niveau du primaire, c'est durant les trois premières années qu'on observe le plus d'absences et plus particulièrement à la première année. Au secondaire c'est aux dernières années de chaque cycle qu'il y a le plus d'absences et particulièrement à la sixième année (tableau 25).

Tableau 25 : Proportions (en \%) des absences selon le niveau et la classe

\begin{tabular}{cccc}
\hline \multirow{2}{*}{ Classe } & \multicolumn{3}{c}{ Niveau au moment de l'absence } \\
\cline { 2 - 4 } & Pré-primaire & Primaire & Secondaire \\
\hline 1 & 6,3 & 3,0 & 2,4 \\
2 & 0,0 & 2,8 & 3,8 \\
3 & 0,5 & 3,0 & 2,0 \\
4 & NA & 2,6 & 2,1 \\
5 & NA & 2,8 & 9,0 \\
6 & NA & 2,5 & 37,1 \\
\hline Ensemble & 1,4 & 2,8 & 3,0 \\
\hline
\end{tabular}

Source : Données de l'enquête auprès des ménages, EADE-RDC 2012

NA : Non applicable

\section{c) Durée moyenne des absences}

Selon l'enquête EADE, les durées moyennes d'absence varient entre 4 et 7,3 semaines. Les durées moyennes d'absence sont plus longues dans les villes (5,6 semaines en moyenne) comparativement au milieu rural (4,8 semaines). Cette situation est assez générale sauf au Bandundu et dans une moindre mesure dans les deux Kasaï (tableau 26). C'est dans les provinces du Bandundu, du Maniema, et de Kinshasa que les durées d'absence sont les plus longues.

Tableau 26 : Durée moyenne d'absence en nombre de semaines par province selon le milieu

\begin{tabular}{lrcc}
\hline Provinces & Urbain & Rural & Ensemble \\
\hline Kinshasa & 6,0 & - & 6,0 \\
Bas-Congo & 5,4 & 5,5 & 5,5 \\
Bandundu & 5,7 & 7,3 & 6,7 \\
Equateur & 5,3 & 4,4 & 4,5 \\
Province orientale & 5,7 & 4,8 & 5,0 \\
Nord-Kivu & 5,5 & 3,8 & 4,1 \\
Maniema & 8,4 & 5,4 & 6,2 \\
Sud-Kivu & 5,3 & 4,6 & 4,7 \\
Katanga & 5,6 & 4,5 & 5,1 \\
Kasaï oriental & 4,3 & 4,8 & 4,6 \\
Kasaï occidental & 4,8 & 5,2 & 5,1 \\
\hline Ensemble & 5,6 & 4,8 & 5,1 \\
\hline Sorce : Donne
\end{tabular}

Source : Données de l'enquête auprès des ménages, EADE-RDC 2012 


\subsubsection{Population à risque d'abandonner}

Comme mentionné précédemment, il existe plusieurs facteurs qui contribuent à accroître le risque d'abandonner pour un enfant ou un adolescent scolarisé. Pour estimer la population à risque d'abandonner, nous avons considéré les élèves qui répondaient à l'un ou l'autre des critères suivants (certains pouvant en combiner plusieurs à la fois) :

- un retard scolaire de 2 ans et plus ;

- une entrée tardive à l'école (au-delà de 7 ans) ;

- être au travail selon la définition de UCW ;

- connaître des périodes d'absences au cours de l'année scolaire 2010-2011 ;

- $\quad$ être entré au primaire sans avoir fait la maternelle (ECCE)

Sur cette base nous avons estimé à 2667319 les élèves de la dimension 4 (6-11 ans) à risque d'abandonner, ce qui correspond à $27,8 \%$ des élèves de cette tranche d'âge scolarisés en 2011-2012. Ces chiffres par sexe sont de 1397435 (27,7\%) pour les garçons et 1269884 (27,9\%) pour les filles. Au niveau de la population à risque d'abandonner de la dimension 5 (12-13 ans), on a une estimation de 2182412 , soit $64,1 \%$ des 12 13 ans scolarisés en 2011-2012. Des différences existent à ce niveau entre filles et garçons : les premières sont 66,0 \% (1 029 472) à être à risque contre 62,5\% (1 152 940) pour les derniers.

II apparaît que quelle que soit la dimension (4 ou 5), les élèves à risque d'abandonner sont proportionnellement plus nombreux en milieu rural $(78,6 \%)$. Lorsqu'on répartit par province la population à risque d'abandonner, on observe que plus de $50 \%$ se retrouvent dans quatre provinces : la Province orientale $(15,2 \%)$, le Katanga (14,7 \%), le Bandundu (13,1\%) et l'Equateur (10,1\%). C'est au Maniema (3,1\%) qu'il y a le plus faible pourcentage de population à risque d'abandonner. Cette répartition se maintient quels que soient la dimension et le sexe (tableau 27).

C'est dans les ménages avec moins de 50 USD de revenu mensuel que l'on trouve plus de la moitié des enfants à risque d'abandonner (55,1\%). On trouve plus d'enfants à risque d'abandonner appartenant à des ménages dont le chef n'a aucun niveau d'instruction. Environ deux enfants sur cinq appartiennent à un ménage dirigé par une femme. Les enfants à risque d'abandonner sont relativement plus nombreux dans les ménages ayant 3-4 enfants de 6-17 ans. 
Tableau 27 : Profil de la population à risque d'abandonner (en \%)

\begin{tabular}{|c|c|c|c|c|c|c|c|c|c|}
\hline \multirow{2}{*}{ Caractéristiques } & \multicolumn{3}{|c|}{ 6-11 ans } & \multicolumn{3}{|c|}{$12-13$ ans } & \multicolumn{3}{|c|}{$6-13$ ans } \\
\hline & Garçons & Filles & Ensemble & Garçons & Filles & Ensemble & Garçons & Filles & Ensemble \\
\hline \multicolumn{10}{|l|}{ Milieu de résidence } \\
\hline Urbain & 22,8 & 19,5 & 21,2 & 22,5 & 20,5 & 21,6 & 22,7 & 20,0 & 21,4 \\
\hline Rural & 77,2 & 80,5 & 78,8 & 77,5 & 79,5 & 78,4 & 77,3 & 80,0 & 78,6 \\
\hline Total & 100,0 & 100,0 & 100,0 & 100,0 & 100,0 & 100,0 & 100,0 & 100,0 & 100,0 \\
\hline \multicolumn{10}{|l|}{ Provinces } \\
\hline Kinshasa & 7,9 & 6,0 & 7,0 & 6,0 & 3,7 & 4,9 & 7,1 & 5,0 & 6,0 \\
\hline Bas-Congo & 8,6 & 7,6 & 8,1 & 6,9 & 7,3 & 7,1 & 7,8 & 7,4 & 7,6 \\
\hline Bandundu & 13,3 & 13,4 & 13,4 & 13,3 & 12,4 & 12,9 & 13,3 & 13,0 & 13,1 \\
\hline Equateur & 8,3 & 9,5 & 8,9 & 10,0 & 13,3 & 11,6 & 9,0 & 11,2 & 10,1 \\
\hline Orientale & 14,1 & 17,4 & 15,7 & 16,3 & 13,0 & 14,7 & 15,0 & 15,5 & 15,2 \\
\hline Nord Kivu & 8,0 & 7,9 & 8,0 & 6,9 & 8,8 & 7,8 & 7,5 & 8,3 & 7,9 \\
\hline Maniema & 2,9 & 3,3 & 3,1 & 3,3 & 2,8 & 3,1 & 3,1 & 3,1 & 3,1 \\
\hline sud Kivu & 8,2 & 8,1 & 8,1 & 6,7 & 7,6 & 7,1 & 7,5 & 7,9 & 7,7 \\
\hline Katanga & 15,4 & 12,9 & 14,2 & 15,4 & 15,4 & 15,4 & 15,4 & 14,0 & 14,7 \\
\hline Kasaï oriental & 7,4 & 8,0 & 7,7 & 9,7 & 8,3 & 9,0 & 8,4 & 8,1 & 8,3 \\
\hline Kasaï occidental & 6,0 & 6,0 & 6,0 & 5,6 & 7,5 & 6,5 & 5,8 & 6,7 & 6,2 \\
\hline Total & 100,0 & 100,0 & 100,0 & 100,0 & 100,0 & 100,0 & 100,0 & 100,0 & 100,0 \\
\hline \multicolumn{10}{|c|}{ Revenu mensuel du ménage } \\
\hline Moins de 50 USD & 54,1 & 56,8 & 55,4 & 55,6 & 53,9 & 54,8 & 54,7 & 55,5 & 55,1 \\
\hline 50 à 100 USD & 27,3 & 26,9 & 27,1 & 27,3 & 31,8 & 29,4 & 27,3 & 29,0 & 28,1 \\
\hline 101 à 200 USD & 11,8 & 10,7 & 11,3 & 11,8 & 9,8 & 10,9 & 11,8 & 10,3 & 11,1 \\
\hline Plus de 200 USD & 6,8 & 5,6 & 6,2 & 5,3 & 4,5 & 4,9 & 6,2 & 5,1 & 5,7 \\
\hline Total & 100,0 & 100,0 & 100,0 & 100,0 & 100,0 & 100,0 & 100,0 & 100,0 & 100,0 \\
\hline \multicolumn{10}{|l|}{ Statut familial } \\
\hline Enfant du CM & 81,2 & 79,4 & 80,3 & 80,0 & 78,5 & 79,3 & 80,7 & 79,0 & 79,9 \\
\hline Autre parent & 18,8 & 20,5 & 19,6 & 19,8 & 21,0 & 20,4 & 19,2 & 20,8 & 20,0 \\
\hline Sans parenté & - & 0,1 & - & 0,2 & 0,4 & 0,3 & 0,1 & 0,2 & 0,2 \\
\hline Total & 100,0 & 100,0 & 100,0 & 100,0 & 100,0 & 100,0 & 100,0 & 100,0 & 100,0 \\
\hline \multicolumn{10}{|l|}{ statut d'orphelin } \\
\hline Non orphelin & 89,1 & 88,8 & 88,9 & 84,9 & 86,4 & 85,6 & 87,2 & 87,8 & 87,5 \\
\hline Orphelin de mère & 2,2 & 2,9 & 2,5 & 3,1 & 4,0 & 3,6 & 2,6 & 3,4 & 3,0 \\
\hline Orphelin de père & 6,6 & 6,7 & 6,6 & 10,2 & 7,4 & 8,9 & 8,2 & 7,0 & 7,6 \\
\hline Orphelin complet & 2,2 & 1,6 & 1,9 & 1,8 & 2,1 & 1,9 & 2,0 & 1,8 & 1,9 \\
\hline Total & 100,0 & 100,0 & 100,0 & 100,0 & 100,0 & 100,0 & 100,0 & 100,0 & 100,0 \\
\hline \multicolumn{10}{|c|}{ Niveau d'instruction du CM } \\
\hline Aucun & 56,9 & 60,3 & 58,5 & 53,4 & 50,2 & 51,8 & 55,4 & 55,9 & 55,6 \\
\hline Primaire & 15,6 & 14,1 & 14,9 & 16,1 & 17,2 & 16,6 & 15,8 & 15,4 & 15,6 \\
\hline Secondaire+ & 27,5 & 25,6 & 26,6 & 30,5 & 32,7 & 31,6 & 28,8 & 28,7 & 28,7 \\
\hline Total & 100,0 & 100,0 & 100,0 & 100,0 & 100,0 & 100,0 & 100,0 & 100,0 & 100,0 \\
\hline \multicolumn{10}{|c|}{ Sexe du chef de ménage } \\
\hline Hommes & 60,9 & 58,8 & 59,8 & 64,8 & 62,8 & 63,8 & 62,6 & 60,5 & 61,6 \\
\hline Femmes & 39,1 & 41,2 & 40,2 & 35,2 & 37,2 & 36,2 & 37,4 & 39,5 & 38,4 \\
\hline Total & 100,0 & 100,0 & 100,0 & 100,0 & 100,0 & 100,0 & 100,0 & 100,0 & 100,0 \\
\hline \multicolumn{10}{|c|}{ nombre d'enfants de 6-17ans } \\
\hline $1-2$ & 41,7 & 43,6 & 42,6 & 34,7 & 36,8 & 35,7 & 38,6 & 40,7 & 39,6 \\
\hline $3-4$ & 46,7 & 44,2 & 45,5 & 48,3 & 48,3 & 48,3 & 47,4 & 46,0 & 46,7 \\
\hline 5 et plus & 11,6 & 12,1 & 11,9 & 17,0 & 14,8 & 16,0 & 14,0 & 13,3 & 13,6 \\
\hline Total & 100,0 & 100,0 & 100,0 & 100,0 & 100,0 & 100,0 & 100,0 & 100,0 & 100,0 \\
\hline
\end{tabular}

Source : Données de l'enquête auprès des ménages, EADE-RDC 2012

Le graphique 11 donne une vue synthétique des différents profils des EADE. Les filles sont proportionnellement plus nombreuses $(31,8 \%)$ que les garçons $(26,6 \%)$ à être en dehors de l'école ; les 
enfants du milieu rural sont proportionnellement plus nombreux (33,4 \%) que ceux résidant en milieu urbain $(20,0 \%)$. Par ailleurs, les inégalités en termes de participation scolaire sont importantes entre les enfants des catégories les plus pauvres (36,6\% d'EADE) et ceux des catégories les plus nanties (11,2\% d'EADE), d'une part ; et entre les enfants dont le chef de ménage n'a aucun niveau d'instruction (32,9\% d'EADE) et ceux dont de chef de ménage a le niveau secondaire ou plus (18,7\% d'EADE), d'autre part. Mais ce qui est important à retenir, c'est que le cumul de ces inégalités montre combien les efforts à faire sont énormes : en effet, pour les filles des ménages ayant moins de 50 USD par mois, dont le chef de ménage n'a aucun niveau d'instruction et résidant en milieu rural, la proportion des EADE est de 45,0 \% contre 37,6\% pour les garçons dans la même situation.

Graphique 11 : Profil scolaire des enfants et adolescents de 5-17 ans en 2012 selon certaines caractéristiques (en $\%)$

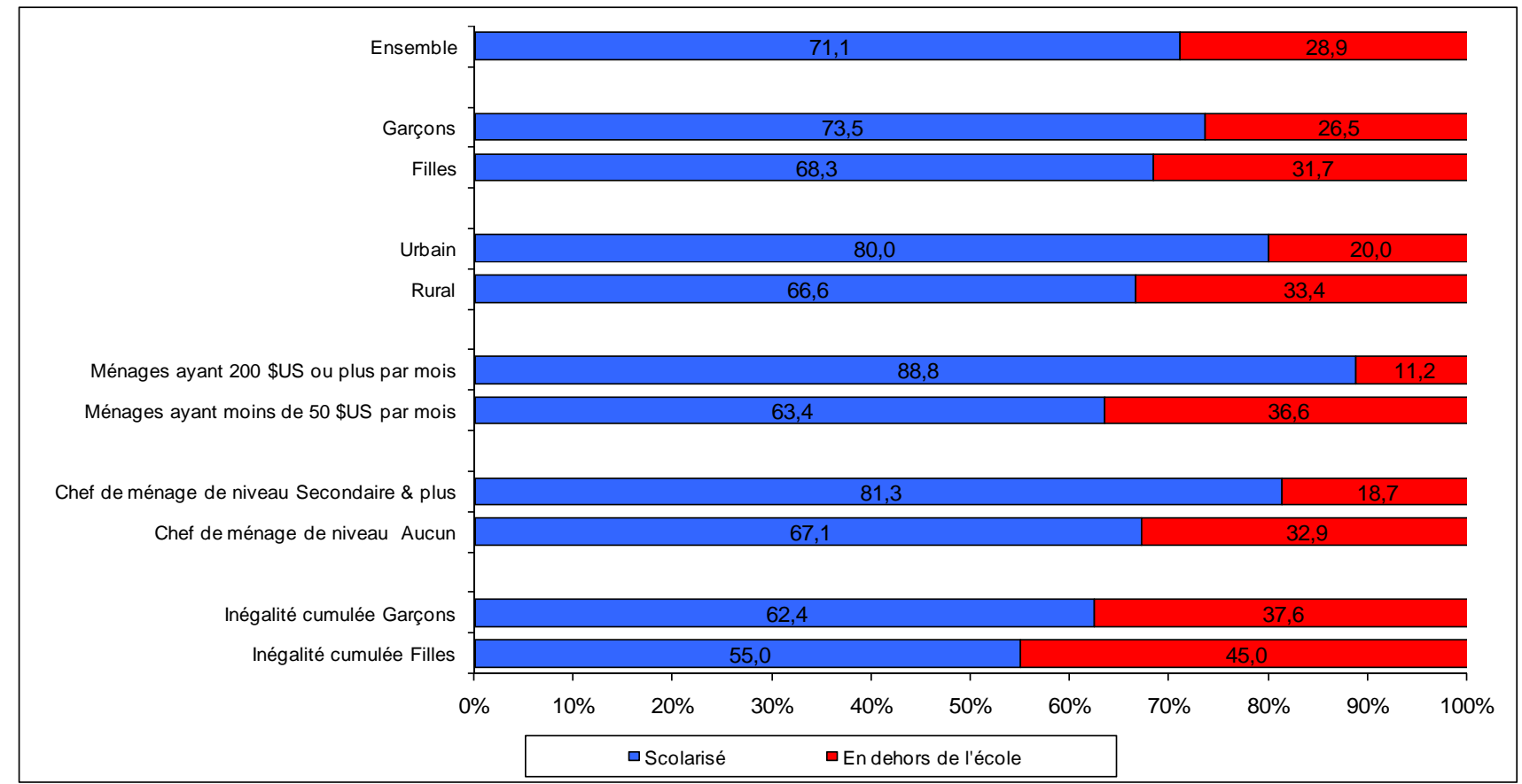

Source : Données de l'enquête auprès des ménages, EADE-RDC 2012

Le tableau 28 résume l'ampleur du phénomène des EADE, dans une perspective de comparaison internationale sur la base des trois tranches d'âge des cinq dimensions de l'exclusion.

Tableau 28 : Synthèse de l'ampleur des EADE selon les cinq dimensions

\begin{tabular}{|c|c|c|c|c|c|c|c|c|c|}
\hline \multirow{2}{*}{$\begin{array}{c}\text { Age de } \\
\text { scolarisation }\end{array}$} & Garçons & Filles & Ensemble & Garçons & Filles & Ensemble & Garçons & Filles & Ensemble \\
\hline & \multicolumn{3}{|c|}{ Population scolarisable } & \multicolumn{3}{|c|}{ Population des EADE } & \multicolumn{3}{|c|}{ Rapport } \\
\hline 5 ans & 1376623 & 1343458 & 2720081 & 1091943 & 1052252 & 2144195 & 79,3 & 78,3 & 78,8 \\
\hline 6-11 ans & 6791505 & 6326092 & 13117597 & 1749792 & 1759460 & 3509252 & 25,8 & 27,8 & 26,8 \\
\hline $12-13$ ans & 2047336 & 1870609 & 3917945 & 203408 & 309759 & 513167 & 9,9 & 16,6 & 13,1 \\
\hline \multirow[t]{2}{*}{ Total } & 215464 & 9540159 & 19755623 & 3045143 & 3121471 & 6166614 & 29,8 & 32,7 & 31,2 \\
\hline & \multicolumn{3}{|c|}{ Population scolarisée } & \multicolumn{3}{|c|}{ Population à risque d'abandonner } & \multicolumn{3}{|c|}{ Rapport } \\
\hline 6-11 ans & 5041713 & 4566633 & 9608346 & 1397435 & 1269884 & 2667319 & 27,7 & 27,8 & 27,8 \\
\hline $12-13$ ans & 1843928 & 1560850 & 3404778 & 1152940 & 1029472 & 2182412 & 62,5 & 66,0 & 64,1 \\
\hline Total & 6885641 & 6127483 & 13013124 & 2080692 & 1890781 & 3971473 & 30,2 & 30,9 & 30,5 \\
\hline
\end{tabular}

Source : Données de l'enquête auprès des ménages, EADE-RDC 2012 


\section{Résumé analytique :}

L'objectif de ce premier chapitre était de dresser le profil des enfants et adolescents en dehors de l'école, notamment de répondre aux trois premières questions de recherche, à savoir : combien sont-ils ?, où sont-ils ?, et qui sont-ils ?

En termes d'ampleur du phénomène des EADE, il ressort que celle-ci reste encore énorme, bien qu'on ait observé au fil des dernières années un accroissement de la participation scolaire des enfants et des adolescents. En effet, plus d'un enfant ou adolescent sur quatre (28,9\%) âgé de 5-17 ans est en dehors de l'école en 2012, soit en termes absolus, 7375876 enfants et adolescents. Sur la période de cinq ans (entre l'EDS de 2007 et l'enquête EADE de 2012), le rythme moyen annuel de baisse de la proportion des EADE a été de $1,92 \%$ par an. Si les tendances actuelles se poursuivent, il faudra attendre encore une quinzaine d'années (donc en 2027) pour voir se résorber définitivement le phénomène des EADE.

L'ampleur du phénomène des enfants et adolescents en dehors de l'école est la plus forte chez les filles ( 31,7 $\%$ ) que chez les garçons (26,5\%). En termes absolus, les filles représentent plus de la moitié (soit $52,7 \%$ ) des EADE. Par ailleurs, c'est dans la tranche d'âges de la scolarisation obligatoire (6-11 ans) que l'effectif des EADE est le plus élevé, soit $47,6 \%$ des EADE.

En termes de localisation géographique, c'est en milieu rural qu'on retrouve le plus grand nombre d'EADE, soit $77,2 \%$. C'est aussi en milieu rural que la proportion d'EADE est plus forte $(33,4 \%)$ contre $20,0 \%$ en milieu urbain. L'analyse spatiale de l'ampleur du phénomène révèle que c'est au Nord Kivu qu'il y a proportionnellement plus d'enfants d'âges scolaires en dehors de l'école $(43,9 \%$, suivis du Katanga $(34,8$ $\%)$, du Kassaï occidental (32,4\%), de la province orientale (32,2 \%), du Sud-Kivu (30,3\%) et du Kassaï oriental (29,3\%). En termes absolus, c'est le Katanga qui vient en tête avec 1334876 enfants de 5-17 ans en dehors de l'école, suivis de la Province orientale (1 039 858), du Nord Kivu (994 366) et de l'Equateur (726 194). C'est donc dans les provinces à forte production minière et celles où les conflits sont récurrents, que l'ampleur du phénomène est la plus forte.

En termes de profil, il ressort que les EADE sont davantage dans des ménages à faible revenu $(56,1 \%$ des enfants en dehors de l'école âgés de 5 ans, 64,3\% des enfants en dehors de l'école âgés de 6-11 ans, 64,9\% des enfants en dehors de l'école âgés de 12-13 ans et 60,2\% des adolescents en dehors de l'école âgés de 14-17 ans) ; qu'ils résident davantage dans des ménages dont le chef n'a aucun niveau d'instruction (65,3\% des enfants en dehors de l'école âgés de 6-11 ans, 46,5\% des enfants en dehors de l'école âgés de 12-13 ans et 50,8\% des adolescents en dehors de l'école âgés de 14-17 ans) et qu'ils sont surtout, à partir des âges du secondaire, des filles $(60,4 \%$ des enfants en dehors de l'école âgés de $12-13$ ans et $63,8 \%$ de ceux âgés de 14-17 ans).

Plus encore, cumuler cet ensemble d'inégalités, constitue un critère important à prendre en compte lors de l'identification des groupes cibles : en effet, pour les filles des ménages les plus pauvres, dont le chef de ménage n'a aucun niveau d'instruction et résidant en milieu rural, la proportion des EADE est de 45,0 \% contre $37,6 \%$ pour les garçons dans la même situation.

Ceci donne une idée de l'ampleur des efforts qu'il y aura à faire pour ramener tous les enfants et adolescents à l'école. Les défis sont encore énormes et exigent une bonne identification des barrières et goulots d'étranglement, en vue de s'attaquer aux causes profondes de l'exclusion scolaire. 


\section{Barrières et goulots d'étranglement}

La revue de la littérature révèle de nombreuses barrières tant au niveau de l'offre que de la demande qui limitent la scolarisation des enfants et des adolescents. Nous commençons ce chapitre par énumérer quelles sont ces barrières généralement citées, ensuite dans le cas de la RDC et à partir des données collectées dans le cadre de l'étude EADE, nous présentons celles qui apparaissent les plus déterminantes dans l'explication de l'exclusion des enfants et des adolescents du système scolaire.

\subsection{Barrières à la scolarisation des enfants et adolescents}

\subsubsection{Barrières relevant de l'offre scolaire}

La disponibilité et la proximité des infrastructures éducatives, leurs équipements, leur accessibilité financière, les qualifications du corps enseignant, les contenus des enseignements et leur adaptabilité, etc. sont autant de facteurs qui ressortent des études antérieures comme pouvant influencer la propension des familles ou des parents à envoyer les enfants à l'école ou leur choix du type l'école.

La question de la distance à l'école intervient souvent dans l'explication des inégalités sexuelles d'accès à l'école. Les parents évitent souvent d'envoyer les filles dans des écoles distantes du lieu d'habitation de crainte de les exposer à des agressions physiques ou morales: "In Egypt, Morocco, and Tunisia parents are reluctant to send their daughters to distant schools because they fear exposing them to moral or physical peril. Even in relatively more open societies of Malaysia and the Philippines distance to school is a greater deterrent to girls' enrollment than to boys' » (Hill et King, 1993, p. 33; Banque Mondiale, 2003; Durand, 2006; Diallo, 2006; Banque Mondiale, 2011). Une étude menée au Burkina Faso révèle que la distance à l'école est le premier déterminant de la scolarisation des enfants en milieu rural et cet effet de la distance est plus important pour les filles que pour les garçons (Kobiané, 2006).

Alderman et al. (1996) mettent également en évidence, dans le contexte du milieu rural pakistanais, que la disponibilité des infrastructures scolaires est l'un des facteurs déterminants de l'accès et des performances des filles à l'école. La disponibilité de certains équipements à l'école est aussi un facteur qui peut limiter les chances de scolarisation des filles : "In Bangladesh parents have withdrawn girls but not boys from schools without latrines » (Hill et King, 1993, p. 33).

La qualité de l'école et les coûts de la scolarisation sont aussi des déterminants de la décision des familles les plus pauvres à envoyer leurs enfants à l'école ou à choisir entre institutions scolaires publiques et privées : " ... schooling choices of poor households are sensitive to government and private school fees, distance to school, and school quality. In particular, lowering private school fees or distance will increase private school enrollment of poor children » (Alderman et al. 2001, p. 306).

\subsubsection{Barrières relevant de la demande}

En plus de la disponibilité et de la qualité de l'offre, il existe de nombreux autres facteurs de l'environnement familial qui influent sur les chances de scolarisation des enfants (Chernichovsky, 1985 ; Marcoux, 1994 ; Pilon, 1995 ; Lloyd et Blanc, 1996 ; Buchmann, 2000 ; Buchmann et Hannum, 2001 ; Kobiané, 2001 et 2006.).

\section{a) Barrière liée aux rapports de genre}

Plusieurs éléments d'ordre socio-culturel interviennent dans l'explication des inégalités entre garçons et filles en matière d'investissement scolaire. Parmi ceux-ci, la conception traditionnelle affectant la femme aux activités domestiques, la précocité du mariage pour les jeunes filles, le statut "d'étrangère » de la fille, etc. (King et Hill, 1993 ; Lange, 1998 ; Kobiané, 2007). Amenée à quitter sa famille pour aller se marier, la fille est généralement considérée dans les sociétés africaines, pour la plupart à résidence patrilocale, comme une perpétuelle "étrangère ", en qui il n'est pas "utile d'investir » dans la mesure où cet investissement (notamment scolaire) profitera davantage à sa future belle-famille (Kinda, 1995 ; Roth, 1996 ; Zoungrana et al., 1998). 


\section{b) Statut d'orphelin}

De nombreux travaux montrent que la perte des parents est généralement préjudiciable à la scolarisation des enfants (Marcoux et al., 2005 ; Wakam, 2002 ; Ainsworth et Filmer, 2002 ; Case et al. , 2004 ; Kobiané et al., 2005). Toutefois, d'autres travaux révèlent que les orphelins ne sont pas toujours nécessairement moins scolarisés que les non orphelins et arrivent à la conclusion que les orphelins ont parfois des niveaux de scolarisation aussi élevés et même parfois plus élevés que les non orphelins (Nyangara, 2004 ; Foster et al., 1995 ; Urassa et al., 1997). Ce dernier résultat pouvant s'expliquer dans le contexte africain, par le soutien aux orphelins à l'intérieur de la famille étendue.

\section{c) Le confiage}

Dans la plupart des sociétés africaines, les enfants sont confiés pour plusieurs raisons. Isiugo-lbanihe (1985) distingue cinq types de confiage: (i) le confiage à l'intérieur de la parentèle (envoi des enfants chez des membres de la famille en vue de renforcer les liens de solidarités familiales), (ii) le confiage de crise (à la suite d'une dissolution de la famille du fait d'un décès, un divorce ou une séparation), (iii) le confiage d'alliance ou d'apprentissage (enfants envoyés chez des non apparentés de statut social élevé comme aides ou apprentis, en vue de renforcer des liens sociaux, politiques ou économiques), (iv) le confiage domestique (envoi des enfants comme aides familiaux) et (v) le confiage scolaire (motif de scolarisation). Cette diversité des motivations à la base du confiage fait que la nature de la relation entre le lien de parenté avec le chef de ménage et les chances de scolarisation est ambivalente (Pilon, 2005). La relation peut se révéler positive, c'est-à-dire que le confiage offre aux enfants et adolescents une plus grande chance de scolarisation ou au contraire, par le détournement des enfants vers d'autres activités, le confiage peut réduire les chances de scolarisation.

\section{d) Niveau d'instruction des parents}

Un résultat classique qui ressort des travaux sur les déterminants de la demande scolaire est le lien positif entre le niveau d'instruction des parents et la scolarisation de leurs enfants. Plus les parents sont instruits, plus ils valorisent l'école formelle et plus ils accordent une importance particulière à l'éducation des enfants quel qu'en soit le sexe (Hill et King, 1993).

\section{e) Sexe du chef de ménage}

Contrairement aux résultats observés dans d'autres régions en développement, notamment en Amérique latine (Barros et al., 1997 ; Buvinic et Gupta, 1997), les travaux portant sur l'Afrique subsaharienne arrivent généralement à la conclusion que les femmes chefs de ménage scolarisent plus les enfants que les hommes chefs de ménage (Lloyd et Blanc, 1996 ; Pilon et Clévenot, 1996 ; Wakam, 2002 et 2003, Kobiané, 2003 et 2006). La raison la plus invoquée dans la littérature est que les femmes seraient garantes d'une meilleure allocation des ressources au sein du ménage (De Vreyer, 1993 ; Pilon et Clévenot, 1996 ; Lloyd et Blanc, 1996).

\section{f) Revenu du ménage}

Plusieurs travaux, que ce soit en Afrique subsaharienne ou dans d'autres régions en développement, montrent qu'il y a une relation positive entre le niveau de vie du ménage et la scolarisation des enfants (Marcoux, 1994a ; Shapiro et Tambashe, 1996, 1999, 2000 ; Filmer et Pritchett, 1999). Autrement dit, plus le ménage est aisé, moins les enfants seront en dehors de l'école. Ce qui veut dire que la pauvreté est une barrière à la participation scolaire des enfants et des adolescents.

\section{g) Nombre d'enfants d'âge scolaire}

La relation négative entre la taille de la famille ou du ménage et la scolarisation des enfants a été corroborée par les faits dans certaines régions en développement, notamment dans les pays d'Asie du Sud-Est (Knodel et Wongsith, 1991 ; Sathar et Lloyd, 1993 ; Degraff et al., 1996 ; Anh et al., 1998). Les travaux sur l'Afrique sub-saharienne en revanche, aboutissent à un tout autre résultat : la relation entre la taille de la famille ou du ménage et la scolarisation des enfants est plutôt inexistante, voire positive (Gomes, 1984 ; Chernichovski, 1985 ; Marcoux, 1994 ; Lloyd et Blanc 1996, Shapiro 1999). Cette différenciation entre l'Asie du Sud-Est et l'Afrique sub-saharienne quant à la nature de la relation entre la taille du ménage et le niveau de scolarisation des enfants est généralement expliquée par des différences dans les systèmes familiaux. L'existence de réseaux de solidarités familiales en Afrique sub-saharienne, qui permettent bien souvent l'accueil de personnes extérieures au ménage (enfants comme adultes) ou l'envoi de certains membres du ménage vers 
d'autres unités résidentielles, aurait pour effet de réduire la pression du nombre d'enfants sur les ressources disponibles.

\section{h) Nombre de malades de longue durée dans le ménage}

Les études portant sur l'effet de la mortalité des adultes sur le bien-être des enfants, notamment dans les zones de forte prévalence de certaines maladies telles que le VIH/SIDA, révèlent que lorsqu'il y a des cas de malades de longue durée, cela peut compromettre la participation scolaire des enfants et des adolescents (particulièrement des filles), et cela à travers deux mécanismes possibles : le coût d'opportunité (ou coût indirect) de la scolarisation des enfants, qui amènerait les ménages à retirer les enfants (et notamment des filles) de l'école pour qu'ils prennent soins de ces malades; ou alors du fait de la compétition sur les ressources, il y aurait davantage une réallocation des revenus vers les soins de santé, ce qui compromettrait la scolarisation des enfants ${ }^{12}$ (Steinberg et al., 2002 ; Yamano et Takashi, 2004).

Sur la base de ces éléments qui ressortent de la littérature sur les obstacles et les goulots d'étranglement dans la scolarisation, analysons à présent ce qu'il en est en RDC.

\subsection{Déterminants de l'exclusion scolaire des enfants et des adolescents en RDC}

Nous avons dans un premier temps examiné la relation entre chaque barrière potentielle (variable indépendante) et l'exclusion scolaire, donc réalisé des analyses bivariées. Les résultats de ces analyses bivariées avec les mesures d'association associées ${ }^{13}$ sont présentés dans les tableaux A24 et A25 en annexe.

Mais comme évoqué plus haut dans la section sur les méthodes d'analyse, pour mieux appréhender les déterminants de l'exclusion scolaire, une analyse multivariée s'impose. Elle a l'avantage de permettre la prise en compte simultanée de l'ensemble des facteurs possibles et de donner leur poids respectif dans la prédiction du risque d'être en dehors de l'école.

Sur la base des résultats de la littérature sur les barrières de la demande d'éducation, les variables suivantes ont été prises en compte dans l'analyse multivariée ${ }^{14}$ :

- le sexe de l'enfant;

- le statut d'orphelin;

- le lien de parenté avec le chef de ménage ;

- le niveau d'instruction du chef de ménage ;

- le sexe du chef de ménage ;

- le revenu du ménage ;

- le nombre d'enfants d'âge scolaire (6-17 ans) dans le ménage ;

- le nombre de malades de longue durée dans le ménage au cours des 12 derniers mois ;

- la distance à l'école.

Etant donné que les déterminants de l'exclusion scolaire peuvent varier d'un groupe d'âges scolaire à un autre mais aussi d'un milieu de résidence à un autre, la régression logistique a été réalisée à plusieurs niveaux :

- $\quad$ un modèle pour les enfants d'âge scolaire du primaire (6-11 ans) et un autre pour les enfants d'âge scolaire du secondaire (12-17 ans) ;

- $\quad$ un modèle pour l'ensemble de la RDC, mais aussi un modèle pour le milieu urbain et un autre pour le milieu rural ;

- $\quad$ un modèle pour chaque province.

Nous n'avons pas réalisé d'analyse multivariée pour la population âgée de 5 ans compte tenu du très faible niveau de participation scolaire à cet âge mais aussi parce que la participation au pré-primaire est

\footnotetext{
${ }^{12}$ C'est la raison pour laquelle dans le cadre de l'étude EADE-RDC, nous avons tenté de mesurer l'effet du nombre de malades de longue durée (maladie ayant durée au moins 3 mois) au cours des 12 derniers mois sur l'exclusion scolaire.

${ }^{13}$ Les mesures ou coefficients d'association sont des statistiques permettant de tester l'existence d'une relation entre deux variable, son intensité et éventuellement (pour des variables de niveau de mesure au minimum ordinal), la direction de la relation. Sur la base de la littérature et des avantages et limites des mesures d'association, nous avons opté pour le $\mathrm{V}$ de Cramer pour des variables de niveau de mesure nominal et le D de Somers pour des variables de niveau de mesure ordinal. Nous avons considéré le fait d'être en dehors de l'école ou pas (bien que dichotomique) comme une variable ordinale. Pour plus de détails sur les mesures d'association, voir notamment W. Fox (1999) ou A. Gilles (1994).

${ }^{14}$ La variable handicap bien que d'intérêt, n'a pas été prise en compte dans les analyses multivariées à cause des faibles effectifs qui posent des problèmes en termes de calcul des coefficients de régression pour plusieurs catégories.
} 
essentiellement l'apanage des zones urbaines et des catégories sociales les plus aisées. Toutefois, dans la synthèse, nous reviendrons sur un résumé des barrières au niveau de la population de 5 ans. Par ailleurs, nous n'avons pas distingué les 12-13 ans des 14-17 ans, pour avoir des effectifs suffisants pour les analyses multivariées notamment par province.

Les coefficients des modèles de régression logistique ou rapports de chances sont présentés dans les tableaux A26 et A27 en annexe. En plus de ces coefficients qui permettent d'examiner les écarts entre catégories des différentes variables, la hiérarchisation des variables selon leur pouvoir explicatif permet d'identifier quels sont les déterminants de l'exclusion scolaire au niveau national et dans chaque milieu de résidence ${ }^{15}$. Le tableau A29 donne cette hiérarchisation des variables explicatives du risque d'être en dehors de l'école, aussi bien au niveau de l'ensemble du pays que du milieu de résidence (urbain/rural et provinces) et cela tant pour les 6-11 ans que pour 12-17 ans. Les variables ont été ordonnées tout d'abord au niveau de l'ensemble du pays et ensuite leur positionnement au niveau de l'ensemble est examiné en milieu urbain, en milieu rural et dans chacune des provinces. Pour faciliter l'interprétation des résultats nous avons mis en exergue dans chaque milieu les trois premières variables les plus déterminantes (couleur rouge) et les trois variables les moins déterminantes (couleur bleue).

\subsubsection{Déterminants socio-culturels de la demande et environnement familial}

\section{a) Les inégalités sexuelles}

La hiérarchisation des variables (tableau A29 en annexe) révèle que c'est aux âges de scolarisation du secondaire (12-17 ans) et particulièrement en milieu rural que le sexe apparaît comme un déterminant de l'exclusion scolaire. La variable sexe occupe la troisième place au niveau national et le premier rang en milieu rural. Elle est particulièrement déterminante dans le Kassaï occidental (où elle occupe le premier rang) et dans le Bas Congo et le Nord Kivu (où elle occupe le $3^{\text {ème }}$ rang). L'examen des rapports de chances (tableau A28 en annexe) montre qu'une fille de 12-17 ans en milieu urbain a 1,49 fois de chances de se retrouver en dehors de l'école comparée à un garçon en milieu urbain et 2,28 fois en milieu rural.

Les efforts déployés par l'Etat et ses partenaires dans la scolarisation des enfants ces dernières années a permis de réduire considérablement les inégalités d'accès à l'école entre garçons et filles, notamment au primaire. Grâce aux campagnes de sensibilisation menées sur l'ensemble du territoire, beaucoup de parents ont compris l'intérêt de scolariser aussi bien les garçons que les filles.

"L'accès des filles et des garçons ? Ces derniers temps les filles s'adonnent aux études, plus que même les garçons, elles s'adonnent beaucoup, vous voyez dans le temps passé on disait qu'elles vont finir par se marier, mais maintenant c'est le contraire, vous voyez des femmes qui sont des autorités, si moi je n'avais pas étudié je n'allais pas travailler, je suis chez moi dans mon foyer mais je travaille, parce que si je n'avais pas un peu étudié, je n'allais pas travailler voilà pourquoi vous voyez que beaucoup de filles s'adonnent aux études, comme chez nous ici à l'école, l'effectif qui dépasse chez nous, c'est des filles; il y a plus de filles que de garçons, vous voyez que les écoles de filles sont devenus nombreuses, il y a que des filles, que des filles, beaucoup de parents se sont donnés à inscrire aussi les filles au lieu d'inscrire que des garçons c'est comme ça, les filles se donnent maintenant beaucoup aux études " (Femme, parente, Equateur).

Toutefois, l'inégalité entre filles et garçons augmente au fur et à mesure qu'on avance dans le système éducatif et se pose donc plus au niveau du secondaire et du supérieur.

" Je sais que dans le temps, il y avait une très grande disparité entre la fréquentation des garçons dans les écoles et celle des filles. Mais depuis quelques années, à peu près cinq ou six ans, il y a eu une campagne, très grande campagne pour la scolarisation des filles. Le mot d'ordre a été suivi, beaucoup de filles vont à l'école et même la tendance a été inversée... Au niveau du primaire beaucoup de filles sont à l'école et quand vous comptez le nombre des filles dans les classes, il dépasse celui des garçons. Mais malheureusement cet élan s'estompe à la fin de l'école primaire. Quand on commence l'école secondaire, on trouve que la tendance commence à s'inverser de nouveau, que ce sont les garçons qui continuent aux études secondaires de plus en plus, les filles commencent à abandonner. Et quand on arrive en sixième

\footnotetext{
${ }^{15}$ Cette hiérarchisation des variables (déterminants) dans les différents modèles d'analyse peut se faire à partir du calcul de la contribution de chacune des variables à l'explication du phénomène des enfants et adolescents en dehors de l'école, mesurée par la statistique du Khi-2 du modèle (voir annexe 3 de la note sur la régression logistique et tableau de la statistique du Khi-2).
} 
secondaire, ce sont les garçons qui sont les plus nombreux ». (Homme, cadre du système éducatif, Kasaï Oriental).

Cette importance de la variable sexe au niveau des 12-17 ans reflète assez bien la réalité socio-culturelle, notamment les rapports de genre discriminatoires, qui font qu'à ces âges, les logiques matrimoniales, les grossesses précoces et, d'une manière générale, la place accordée à la fille dans l'organisation familiale, amènent nombre de jeunes filles à quitter l'école. La proportion de filles de 12-17 ans scolarisées et qui sont en union est de $0,08 \%$. Par contre, la proportion de filles de 12-17 en dehors de l'école qui sont en union au moment de l'enquête est de 9,0\%. Même si l'entrée en union peut intervenir après la sortie de l'école et peut ne pas être nécessairement la cause de l'abandon, cette forte proportion des filles EADE en union fait penser à un effet des mariages précoces comme cause de déperdition scolaire chez les filles. Par ailleurs, le tableau A30 en annexe qui donne les causes d'abandon scolaire montre qu'au niveau de l'ensemble du pays, le mariage a été cité dans 3,3\% des cas, et ce chiffre atteint 10,2 \% dans le Maniema, 8,0 \% dans le Kasaï occidental et 7,6 \% dans le Kasaï oriental. De même, la grossesse a été citée dans 3,9\% des cas au niveau de l'ensemble du pays, chiffre qui est de $11,7 \%$ dans le Bandundu, $11,6 \%$ dans le Maniema et $7,0 \%$ dans la Province orientale. Lorsqu'on analyse ces chiffres uniquement pour les filles, ils sont encore plus importants : le mariage est cité dans $6,3 \%$ des cas et la grossesse est citée dans 5,2\% des cas comme raisons d'arrêt de scolarisation pour les filles (tableau A31 en annexe).

Les propos suivants qui ressortent des entretiens qualitatifs, illustrent assez bien le rôle important des logiques familiales :

"Il y a beaucoup de parents qui disent... comme on dit en Lingala " Aza na ye mwana mwasi ako finir naye na libala... ". C'est-à-dire : "étant que fille, elle finira toujours par le mariage ". Alors, ils préfèrent mieux scolariser les garçons pour que demain ou après-demain qu'ils viennent aussi prendre leurs charges [prendre la famille en charge]. » (Femme, parent d'enfant, Kinshasa).

\section{b) La survie des parents}

La perte des parents est une barrière à la scolarisation des enfants et des adolescents, particulièrement en milieu urbain au niveau des 12-17 ans. La hiérarchisation des variables révèle que le statut d'orphelin est le deuxième déterminant de l'exclusion scolaire en milieu urbain chez les $12-17$ ans $\left(6^{\text {ème }}\right.$ déterminant en milieu rural). A Kinshasa, le statut d'orphelin occupe le $1^{\text {er }}$ rang chez les $12-17$ ans et le $3^{\mathrm{eme}}$ rang chez les 6-11 ans. Dans trois autres provinces, Bas Congo, Equateur et Maniema, il est déterminant ( $2^{\text {ème }}$ rang au Bas Congo et à l'Equateur, et $3^{\mathrm{e} \text { me }}$ rang au Maniema).

Les rapports de chances au niveau des 12-17 ans (tableau A28 en annexe) montrent que les orphelins de mère présentent des risques plus élevés d'être en dehors de l'école que les non orphelins, quel que soit le milieu de résidence, avec des écarts encore plus importants que ceux observés chez les 6-11 ans (rapport de chances de 1,96 en milieu urbain contre 1,88 en milieu rural). Par ailleurs, être orphelin des deux parents en milieu rural est un facteur important d'un plus grand risque d'être en dehors de l'école comparativement à un non orphelin (rapport de chances de 1,86).

Le graphique 12 donne la proportion des EADE au sein des 12-17 ans et des 6-11 ans en fonction du statut d'orphelin. Les différences ne sont pas très nettes au niveau national entre les différentes catégories d'orphelin, excepté au niveau des 12-17 ans. L'analyse par milieu de résidence montre toutefois des résultats différents notamment en ce qui concerne la scolarisation des différentes catégories d'orphelin. On retient tout d'abord, que ce soit au niveau des 6-11 ans ou au niveau des 12-17 ans, que c'est chez les non orphelins que la proportion des EADE est la plus faible (pour les 6-11 ans, 17,6 \% en milieu urbain et 30,1\% en milieu rural ; pour les $12-17$ ans, $11,4 \%$ en milieu urbain et $18,3 \%$ en milieu rural). Par contre, en milieu urbain, ce sont les orphelins de mère et les orphelins de père qui présentent les plus fortes proportions des EADE comparativement aux orphelins des deux parents (orphelins complets); alors qu'en milieu rural, ce sont les orphelins des deux parents qui présentent la plus forte proportion des EADE. 
Graphique 12 : Proportion (en \%) d'EADE selon le statut d'orphelin et par groupe d'âge

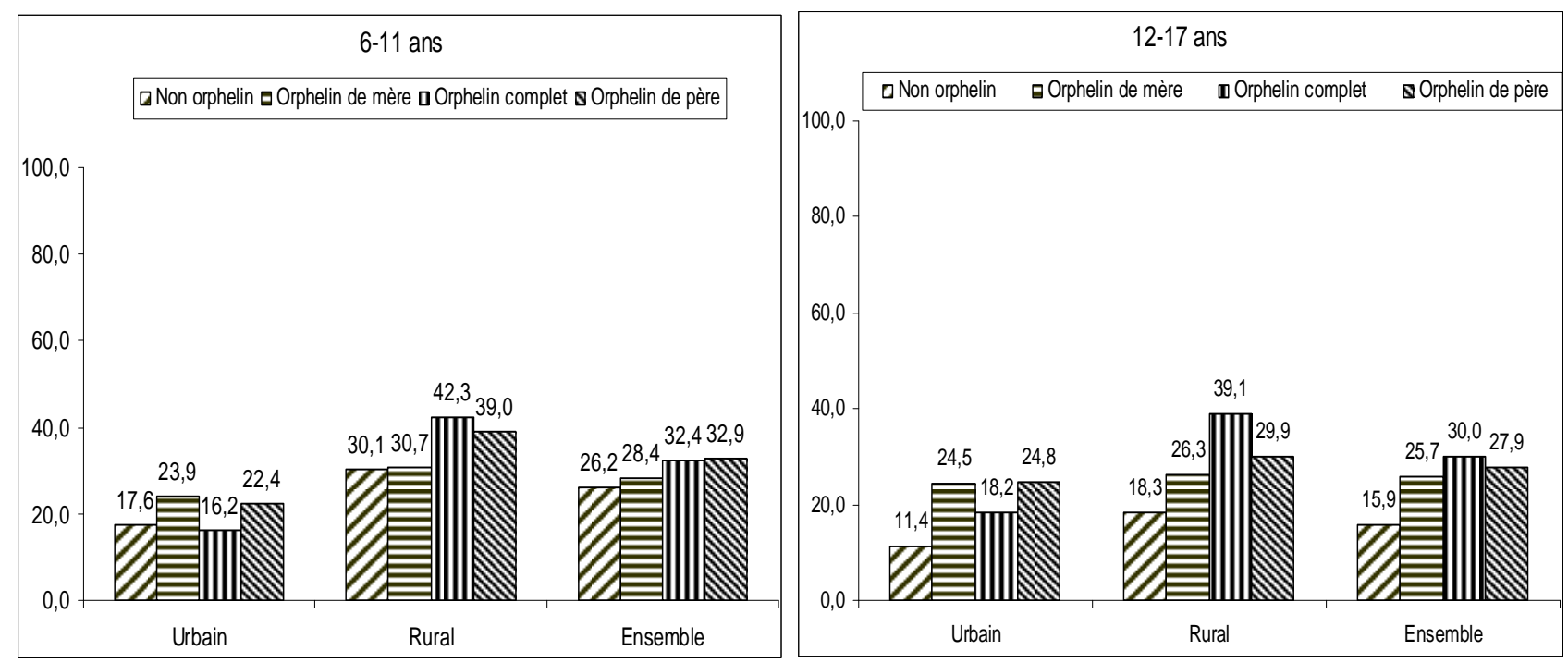

Source : Données de l'enquête auprès des ménages, EADE-RDC 2012

Les propos suivants issues de l'enquête qualitative montrent bien les difficultés de scolarisation auxquelles font face les orphelins :

« Je parlerai, je vous répondrai en tant que veuve moi-même. Moi, je suis veuve, je travaille c'est grâce à mon travail que j'ai scolarisé mes enfants mais, il m'arrive souvent de me poser la question de savoir comment les autres veuves se débrouillent, celles qui n'ont pas de travail ; celles qui n'ont aucune occupation, comment elles se débrouillent pour faire scolariser les enfants et c'est ainsi que vous voyez que la plupart des enfants orphelins sont hors du circuit scolaire à cause de manque de soutien financier (Femme, cadre du système éducatif, Kinshasa). »

"Les enfants orphelins n'ont pas la chance. Vous savez notre société était caractérisée par la solidarité clanique, mais avec le modernisme, la solidarité s'est affaiblie. Mais aussi avec le problème spécifique de notre pays où il y a la misère, c'est difficile de pratiquer la solidarité dans la misère. Alors les enfants orphelins n'ont pas la facilité d'étudier. Dans le cas spécifique de notre école, il y a certaines ONG qui passent prendre la liste des enfants orphelins, il y a certains quand même qui ont la chance, on paie pour eux, comme ceux-là qui sont orphelins du SIDA on essaie de les supporter, mais pas tous. Ce sont ceux-là qui plus ou moins ont trouvé l'occasion de se présenter à l'école. Mais il y a beaucoup d'autres qui sont chez eux à la maison ou même qui se retranchent dans la rue, qui deviennent des enfants en situation de rupture familiale parce que tout simplement ils n'ont pas des parents capables de pouvoir les supporter " (Homme, Enseignant, Nord Kivu).

«Non, non... les enfants orphelins n'ont pas l'accès à l'éducation facile, parce qu'ils n'ont pas d'abord les parents qui peuvent les encourager d'aller étudier et puis les moyens, comment ils peuvent aller étudier, c'est ça! Au moins les enfants qui sont dans des institutions comme dans des centres d'hébergement, comme dans des orphelinats-là, ils étudient » (Femme, institution de prise en charge des enfants, Kasaï oriental). Ceux qui ont la chance d'être récupérés dans les institutions ont des chances de pouvoir accéder à l'école ou poursuivre leur scolarité.

\section{c) Le confiage}

Le confiage est mesuré ici à travers le lien de parenté avec le chef de ménage ${ }^{16}$. Le lien de parenté est apparu comme un déterminant de la participation scolaire, notamment en milieu urbain où il occupe le $3^{\text {ème }}$ rang pour les 6-11 ans et le $4^{\text {ème }}$ rang pour les 12-17 ans. Toujours au niveau des 12-17 ans, cette variable occupe le $1^{\text {er }}$ rang dans le Maniema et le Kasaï occidental et le $3^{\text {ème }}$ rang dans le Bandundu et l'Equateur (tableau A29 en annexe).

L'examen des rapports des chances (tableau A28 en annexe) révèle que plus l'enfant est distant du chef de ménage en termes de parenté, plus il coure le risque de se retrouver en dehors de l'école. Ce résultat

\footnotetext{
${ }^{16}$ Mais il convient de souligner qu'il s'agit d'une mesure indirecte du confiage, car un enfant peut résider dans un ménage qui n'est pas celui de ses parents, sans être confié (par exemple, l'enfant qui est présent dans un ménage avec sa mère).
} 
s'observe aussi bien en milieu rural qu'en milieu urbain, mais est nettement plus important dans ce dernier : un enfant ou un adolescent de 12-17 ans « autre parent » du chef démange a 1,67 fois les chances (risque) d'être en dehors de l'école comparé à un enfant biologique du chef de ménage, et ce risque est cinq fois plus élevé lorsqu'il s'agit d'un enfant non apparenté au chef de ménage.

Ce résultat sur l'effet du lien de parenté avec le chef de ménage montre bien que le confiage des enfants ne contribue pas nécessairement à l'amélioration des chances de scolarisation. Même si parmi les motifs d'envoi, la scolarisation est l'une des raisons généralement évoquée, dans les faits, l'activité que l'enfant peut être amené à faire dans son ménage d'accueil peut être autre que chose que la scolarisation. Les informations collectées lors de l'enquête EADE sur les enfants du ménage vivant ailleurs, montrent que l'envoi des enfants dans d'autres unités résidentielles est un phénomène relativement important : en effet, il ressort de l'enquête que les enfants et adolescents de 5-17 ans vivant en dehors du ménage de leurs parents biologiques représentaient $13,5 \%$ des enfants et adolescents résidant avec leurs parents. Les raisons pour lesquelles les enfants et les adolescents sont envoyés ailleurs sont par ordre d'importance décroissante, pour " fréquenter l'école » $(72,7 \%)$, à cause de la «pauvreté » $(16,5 \%)$, pour « fréquenter et être aide familial » $(7,4 \%)$, pour « autres raisons » $(2,1 \%)$, pour « être aide familial » $(1,3 \%)$.

Les points de vue donnés lors de l'enquête qualitative sur la question de la scolarisation des enfants confiés permettent d'apprécier davantage pourquoi les chances de scolarisation des enfants diminuent lorsqu'ils ne sont plus sous le toit familial :

"Vous savez un enfant est bien éduqué quand il est sous le toit parental, quand il vit avec ses parents. Je veux vous dire que tous ces enfants quand bien même qu'ils peuvent étudier, ils étudient dans quelles conditions ? Aujourd'hui, la ville de Goma connait une difficulté en eau potable, il y a un problème d'eau. Ces enfants doivent se réveiller très tôt le matin, à quatre heure du matin pour parcourir de très longs kilomètres aller chercher de l'eau, l'enfant revient très fatigué, après cela il va se rendre à l'école. Ce sont des enfants qui, dans beaucoup de ménages, sont traités comme des domestiques de la maison et vous devez vous imaginer que les conditions des études deviennent aussi très difficiles (Homme, enseignant, Nord Kivu).

"Pour l'éducation de ces enfants [enfants confiés], il y a un peu de problème parce que certains des enfants en dehors de l'école, ce sont des enfants qui vivent hors [loin] de leurs parents à cause de la pauvreté. Certains membres de famille se déchargent des enfants de leurs proches et de leur famille élargie pour favoriser leurs propres enfants (Femme, personnel ONG national).

"Confiés, mais c'est comme je l'ai dit tantôt. Lorsqu'un enfant est confié à un autre, et si le type auprès de qui on a confié cet enfant n'a pas suffisamment des moyens, s'il a ses propres enfants en âge scolaire, il doit commencer par scolariser n'est-ce pas ses propres enfants. Donc, les enfants qui ont été confiés, courent déjà le risque de se retrouver en dehors du système. A moins qu'ils aient été confiés chez un parent ayant au moins des moyens, et la bonne volonté de pouvoir également les prendre en charge, à coté de ses propres enfants » (Homme, Chargé de la planification et du suivi).

On note également à travers les discussions de groupe avec les enfants et les adolescents, plusieurs propos qui attestent que les enfants confiés ont peu de chances d'être scolarisés par rapport aux autres enfants vivant avec leurs parents biologiques. Les propos suivants relevés lors d'une discussion avec un groupe d'adolescents vivant dans des ménages dans la province du Nord Kivu traduisent l'opinion de beaucoup d'enfants sur les chances de scolarisation des enfants confiés.

(i) "II [enfant confié] n'a pas la même chance que les enfants qu'il va trouver dans cette maison parce que ceux-là qui sont de la maison leur parents vont les aider plus que lui ». (ii) «Pour moi, ils ne sont pas égaux. On va scolariser les autres, mais celui qu'on a confié, on ne va pas le scolariser ". (iii) "Ils ne peuvent pas avoir la même chance. Parce que les parents auront la volonté de scolariser leurs enfants et n'auront pas la volonté de scolariser l'enfant confié. " (iv) "L'enfant confié, on ne va pas le traiter de la même façon que les autres parce que cette maman cherchera que ses enfants en rentrant de l'école, leurs habits soient lavés par l'enfant confié. On va le faire travailler et il n'aura pas le temps d'aller à l'école. ».

Le témoignage ci-dessous d'une adolescente illustre également la situation défavorisée des enfants confiés quant à la réussite scolaire. "Parce que vous voyez, comme certains, moi aussi j'habite chez mon ainée je devais faire l'examen sélectif mais je n'ai pas d'argent pour passer cet examen " (Fille, 13 ans, élève, Équateur). 


\section{d) Perceptions et attitudes des parents sur les droits des enfants}

\section{Perceptions du statut d'enfant}

Les perceptions culturelles de la société dans laquelle vivent les enfants ont un impact sur la formulation de leurs besoins et par conséquent de leurs droits. Elles sont déterminantes aussi bien des attitudes des adultes vis-à-vis des enfants que de leurs pratiques par rapport aux droits des enfants. Pour ce faire, lors des entretiens qualitatifs, les adultes ont été invités à fournir les critères qu'ils utilisent pour différencier l'enfant de l'adulte, leur opinion sur l'idée que les enfants ont des droits, l'égalité des filles et garçons et la nécessité de recueillir l'opinion de l'enfant pour les décisions le concernant.

Les critères de différentiation de l'enfant et de l'adulte relevées par les adultes sont divers. Les critères les plus utilisés sont l'âge, l'irresponsabilité sociale, la dépendance, l'incapacité à prendre et à défendre ses décisions. Ces critères sont soulignés dans les propos suivants. (i) "L'enfant ..., c'est de 0 à 18 ans. L'enfant diffère de l'adulte parce que l'enfant ne se prend pas en charge. Il n'a pas encore de moyens de se prendre en charge, il est un être en devenir, il est sous l'autorité de quelqu'un, il est sous la responsabilité de quelqu'un, c'est quelqu'un qui le sécurise...Mais, l'adulte se prend en charge, il peut poser des actes responsables (Homme, cadre du système éducatif, Kinshasa). (ii) Un adulte peut prendre des décisions de luimême et de même peut peser les pour et les contres. Mais l'enfant ne peut pas prendre une décision qui lui sera propre. Et peut-être aussi, il ne saura pas défendre sa décision (Femme, éducatrice, Kinshasa).

Ces discours montrent que l'enfant est perçu d'une part, comme un être humain en devenir, essentiellement dépendant, car ne pouvant se prendre en charge lui-même et d'autre part comme un être humain à qui on doit transmettre une éducation. La fréquence de ces critères, très spécifiques à l'enfant, permet de prime abord de conclure que la société congolaise a une perception favorable à la promotion des droits de l'enfant.

Toutefois, certains propos précisent que seules les personnes de très bas âge (moins de 12 ans) sont considérées comme enfants. Les personnes âgées de 12 et 18 ans, qualifiées d'adolescents, ne sont plus considérées comme des enfants par certains adultes tel qu'exprimé dans les propos ci-dessous :

"L'enfant je crois de 0, d'une année à 10 ans ; ça c'est un enfant... Un adulte ... quand on parle l'âge de puberté de 15 ans à 20 ans, je crois ça c'est l'âge d'un adolescent je dirai quand nous prenons de 12 ans à 18 ans c'est déjà un adolescent ... je crois quand vous prenez la catégorie de 12 ans à 18 ans c'est un adolescent. Ce n'est pas un enfant; celui-là quand c'est une femme on peut l'engrosser, on ne peut pas engrosser un enfant. Moins de 10 ans, ce n'est pas facile, donc celui-là reste enfant " (Homme, cadre du système éducatif, Équateur).

Une telle perception de l'enfant présente un danger. Le fait de considérer les adolescents comme des adultes et de les soumettre à des traitements d'adulte peut être à l'origine de pratiques préjudiciables à l'enfant comme les mariages précoces, les pires formes de travail des enfants, la prostitution des enfants, les violences sexuelles et l'exploitation économique des enfants. II est donc nécessaire que des actions d'information lèvent toute équivoque quant à l'âge de l'enfant et la nécessité de le protéger jusqu'à l'âge adulte.

Les adultes identifient aussi l'ignorance comme étant un des critères de différentiation entre l'enfant et l'adulte. Pour eux, l'enfant, c'est celui qui ne peut pas bien réfléchir, celui qui ne sait rien. L'adulte raisonne, connait le bien et le mal tandis que l'enfant est encore ignorant de beaucoup de choses (Homme, agent communal, Kinshasa). Cependant, la référence à l'intelligence pour définir un enfant n'est pas pertinent dans la mesure où il peut arriver que l'adulte se situe au même niveau mental que l'enfant. D'ailleurs, une personne interviewée souligne que : "l'enfant raisonne, ça dépend de son âge, il raisonne à la limite de rayon où il se trouve " (Homme, cadre dans l'administration, Kasaï Oriental). Au demeurant, celui qui pense que l'enfant est moins intelligent que l'adulte, ne sera pas prompt à le consulter et à lui demander son avis sur les questions le concernant. Ce qui constitue une violation de ses droits au regard de la CDE et de la loi portant protection de l'enfant. Les actions de sensibilisation devront insister sur les critères spécifiques et pertinents permettant de caractériser l'enfant et combattre les préjugés tendant à dénier de l'intelligence à un enfant.

L'enfant est aussi perçu comme celui qui va assurer la relève, une assurance vieillesse pour les parents comme exprimé dans le discours suivant: " Moi je trouve que l'enfant c'est mon champ.... l'enfant c'est le champ, ... je dois le faire étudier ... je paye les études pour lui. Un jour, quand l'enfant va terminer ses études, l'université, ...il va trouver un bon emploi, au moment où j'ai vieilli, ....cet enfant va penser à moi. II m'appelle papa, prend ceci ou cela, il m'achète un pantalon, il envoie une pièce de pagne à maman...il pensera à moi quand je serai vieux » (Homme, Leader religieux, Équateur). 
L'attitude des adultes vis-à-vis des principes directeurs de la Convention relative aux droits de l'enfant (CDE) L'opinion des personnes enquêtées a été recueillie concernant deux des quatre principes directeurs de la CDE que sont: le principe de non-discrimination et le principe de la participation des enfants. En ce qui concerne l'égalité de droits entre fille et garçon, une forte proportion de répondants estime que les filles et les garçons sont égaux parce que tous les deux sont des personnes à part entière et qu'aucun d'eux n'a demandé à naitre fille ou garçon. Ils doivent donc bénéficier de la même protection que ce soit en famille ou dans la société et être traités de la même manière sans discrimination. Toutefois, comme souligné dans les propos ci-dessous, ils soulignent que la discrimination fondée sur le sexe est présente dans la société congolaise surtout dans les rapports familiaux.

"Les filles sont défavorisées par rapport aux garçons, elles sont vraiment défavorisées. Surtout en matière des travaux domestiques, les travaux domestiques pèsent sur elles. Vous trouvez qu'une fillette-là de 14 ou 15 ans...est devenue comme la mère de famille en lieu et place de sa mère qui doit passer ses journées au marché ou qui doit aller à des kilomètres et des kilomètres amener des marchandises. Sur ce point, les filles vraiment sont défavorisées par rapport aux garçons. II y a des garçons qui sont utilisés en matières économiques, mais ce n'est pas trop. Mais les filles elles, immanquablement dans les travaux domestiques elles sont utilisées. Bien sûr, il y a celles-là qui glissent dans l'immoralité, permettre à la fille de faire la prostitution pour avoir un peu d'argent pour se prendre en charge, ça aussi » (Homme, enseignant, Nord Kivu).

Le principe de la participation de l'enfant ou encore le respect de l'opinion de l'enfant est difficilement admis dans la société congolaise comme dans la plupart des sociétés africaines où seuls les adultes ont le droit de prendre des décisions et de participer au développement de leur communauté. La question sur la nécessité de demander à l'enfant son avis sur toutes les questions le concernant a divisé profondément les personnes interviewées. Il y a ceux qui estiment qu'il faut recueillir l'avis de l'enfant sur toutes les questions le concernant comme le souligne une enseignante : «il faut demander l'avis à l'enfant et l'écouter aussi. Ce qui sort de son intérieur est reçu, mais tamisé par les parents en vue d'une éventuelle orientation pour l'avenir. Ne pas toujours s'imposer. » (Femme, enseignante, Kasaï Oriental). Ils précisent que c'est un droit reconnu à l'enfant qui constitue en même temps un apprentissage de la démocratie. Pour eux, c'est le moyen de mieux connaître ses besoins, ses difficultés et le meilleur moyen d'obtenir l'adhésion de l'enfant aux décisions des parents. Du reste, il faut discuter de tout sujet qui l'intéresse :

"C'est très important, pourquoi ? II faut donner des réponses à toutes les questions posées par l'enfant, pourquoi ? Parce que si l'enfant vous pose la question, si vous voyez que la question va donner une réponse scandaleuse, peut-être vous pouvez avoir honte de répondre. Avec la réponse que vous cachez à l'enfant, l'enfant peut aller ailleurs pour aller découvrir ça ou dans la télé ou soit par des mauvais amis, l'enfant va se rebeller quoi. C'est pourquoi il faut donner des réponses à toutes questions posées par l'enfant, c'est très bien. Il y a des choses qu'on peut faire à l'enfant sans pour autant demander son avis parce qu'il est enfant, de toutes les façons il est enfant. Mais il y a des choses que vous pouvez demander à l'enfant. " (Femme, éducatrice, Nord Kivu).

Par contre, certains ne sont pas favorables à la participation des enfants par crainte de ne pas pouvoir satisfaire les exigences de l'enfant et aussi de perdre leur autorité sur l'enfant. Pourtant, le droit à la participation, tel que défini dans la CDE, ne place pas l'enfant au-dessus de l'autorité ou de la responsabilité parentale. Par ailleurs, la participation dans la prise de décision est progressive, selon l'âge, la capacité et la maturité de l'enfant. Le principe de la participation de l'enfant met en exergue le fait que les enfants sont des personnes jouissant de droits fondamentaux et ayant des opinions et des sentiments qui leur sont propres. Son importance tient au fait qu'il tend à faire de l'enfant un acteur de son propre développement. Les efforts déployés en leur faveur n'aboutissent pas si les intéressés ne sont perçus que comme des personnes à nourrir, à vacciner ou à loger au lieu de personnes à traiter en tant que membres à part entière de leur communauté.

L'attitude des adultes vis-à-vis des droits de l'enfant en général

La Constitution congolaise met à la charge des parents la responsabilité de prendre soin de leurs enfants, de les éduquer et d'assurer leur protection contre tout acte de violence tant à l'intérieur qu'à l'extérieur du foyer. Les parents, donc les adultes, contribuent aux côtés de l'État à l'effectivité des droits de l'enfant. Leur attitude par rapport aux droits de l'enfant est importante. Aussi, est-il intéressant de vérifier si les adultes congolais partagent l'idée que l'enfant, comme tout être humain a des droits et qu'ils admettent que l'enfant a une opinion dont il faut tenir compte. Les résultats montrent que les adultes du milieu rural et urbain s'accordent pour reconnaître presqu'à l'unanimité le principe selon lequel les enfants ont des droits, donc sont sujets de droits. En témoignent ces quelques propos ci-dessous : 
"Les enfants ont des droits parce qu'un enfant, c'est une personne à part entière. Les enfants ont des droits mais il faut seulement aussi prendre le temps de leur apprendre leurs droits. Là on rentre encore dans l'histoire de scolarisation, s'ils ne sont pas instruits comment ils connaitront leurs droits, comment ils connaitront leurs devoirs parce que quand on parle des droits, il faut toujours penser aux devoirs " (Homme, formateur, Nord Kivu)...

"Eh oui, les enfants, comme personnes humaines, ont des droits de la même manière que les adultes et d'autres catégories des personnes. Cette idée est géniale, elle est bonne parce que c'est à partir du moment où il y a existence des droits de l'enfant, à partir du moment où ses droits sont connus par tous, par l'enfant lui-même, par l'adulte et par son entourage, que ses droits sont respectés (Homme, personnel d'une institution de prise en charge des enfants, Nord Kivu).

Toutefois, il y a quelques personnes qui désapprouvent les droits de l'enfant, notamment parmi les religieux et aussi les éducateurs.

"Moi, personnellement ça me choque, je me dis ça doit être d'abord contre la Bible, un enfant qui va accuser les grandes personnes là-bas aux droits des enfants ici à Goma. Moi, j'ai vu ça, ce sont les choses qu'on a imité chez les blancs... Maintenant il y a des droits qui disent qu'il ne faut pas toucher sur un enfant, vous touchez... un enfant, il va vous accuser là-bas, est ce que ça, c'est normal ? Les blancs, ils mettent au monde un enfant ou deux mais nous ici, nous avons 10, 12, 13 et maintenant on dit les droits des enfants, si vous essayez de tirer la joue de l'enfant, il va vous accuser là-bas, vous essayez de faire quoi, il va vous accuser, on dit que ça c'est les droits des enfants, les droits des enfants. Ce n'est pas une bonne idée, c'est à cause de ça, les enfants sont devenus des impolis, sont devenus je ne sais pas quoi, c'est à cause de cela il y a peutêtre même les enfants qui circulent dans la rue chaque jour parce qu'ils ne veulent pas qu'on tonne sur eux [qu'on les gronde], il y a maintenant beaucoup de délinquants à cause de ça " (Femme, éducatrice, Nord Kivu).

\subsubsection{Le capital éducatif comme déterminant de la demande}

Le fait que le chef de ménage n'est pas instruit ou ait un faible niveau d'instruction, constitue une barrière importante à la scolarisation des enfants et des adolescents. Toutefois, on n'observe pas une relation régulière dans le cas de l'enquête EADE 2012 : c'est plutôt chez les chefs de ménage de niveau d'instruction primaire que la proportion des EADE est la plus élevée, suivis des chefs de ménage sans aucun niveau d'instruction. C'est chez les chefs de ménage de niveau d'instruction du secondaire ou plus que la proportion des EADE est la plus faible (graphique 13).

Graphique 13: Proportion (\%) des EADE selon le niveau d'instruction du chef de ménage et par groupe d'âges des enfants
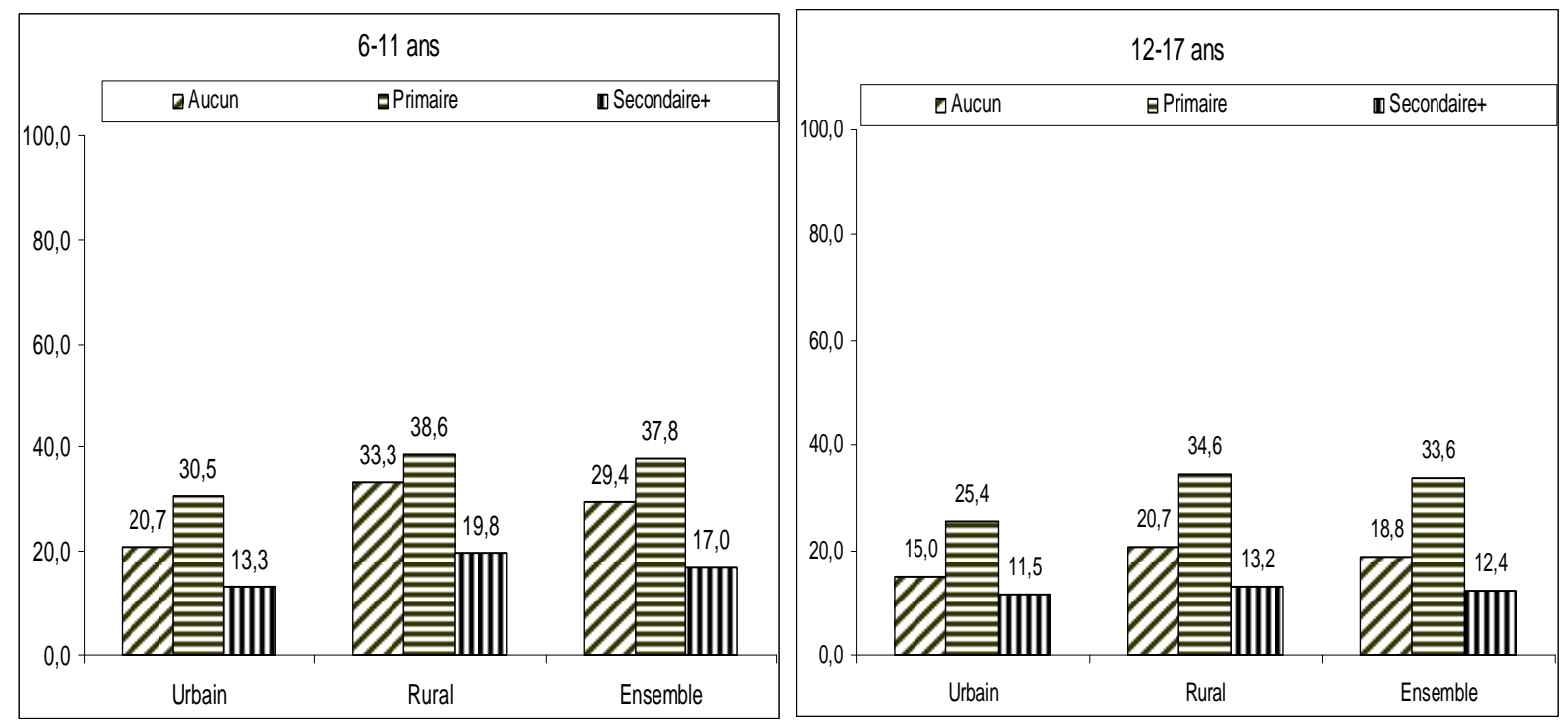

Source : Données de l'enquête auprès des ménages, EADE-RDC 2012 
Le fait que la proportion des EADE soit plus élevée chez les chefs de ménage de niveau d'instruction primaire comparé à ceux sans aucun niveau d'instruction pose question et mériterait plus d'investigation.

Les résultats des analyses multivariées montrent que le niveau d'instruction du chef de ménage est l'un des principaux déterminants de l'exclusion scolaire, puisqu'il vient en $3^{\mathrm{e} m e}$ position dans la hiérarchisation des variables au niveau des 6-11 ans et en $1^{\text {ère }}$ position chez les 12-17 ans. Que ce soit en milieu urbain ou en milieu rural, le niveau d'instruction du chef de ménage est un déterminant majeur des chances de participation scolaire des enfants et adolescents (tableau A29 en annexe). Ce résultat est aussi confirmé par les propos suivants provenant des entretiens qualitatifs :

"Il y a les parents qui préfèrent par exemple utiliser leur argent à autres choses que payer par exemple les frais pour leurs enfants, ça c'est dans des milieux ruraux. Mais en ville, là on voit les parents qui sont intellectuels, qui ont étudié et tout. Et disent [veulent] que leurs enfants puissent suivre leurs pas. " (Femme, éducatrice, Kinshasa).

\subsubsection{Déterminants économiques de la demande scolaire}

a) Revenu du ménage/coût de la scolarisation pour les ménages

Nous commençons d'abord par donner quelques informations sur la distribution de la variable revenu du ménage. Le graphique 14 montre qu'au niveau national, plus de la moitié des ménages congolais $(56,9 \%)$ ont un revenu de moins de 50 USD par mois et un ménage sur cinq $(24,8 \%)$ a un revenu mensuel compris entre 50 et 100 USD. La différence entre villes et campagnes en termes de distribution des revenus est très nette. En milieu rural, $70,4 \%$ des ménages ont moins de 50 USD par mois contre $24,2 \%$ en milieu urbain. C'est par ailleurs en ville qu'on retrouve davantage les ménages de gros revenus, puisque près d'un ménage sur cinq $(18,1 \%)$ en milieu urbain a un revenu mensuel compris entre 201 et 500 USD et près de $4 \%$ ont un revenu entre 501 et 1000 USD $^{17}$.

Graphique 14 : Distribution (en \%) des classes de revenu par milieu de résidence

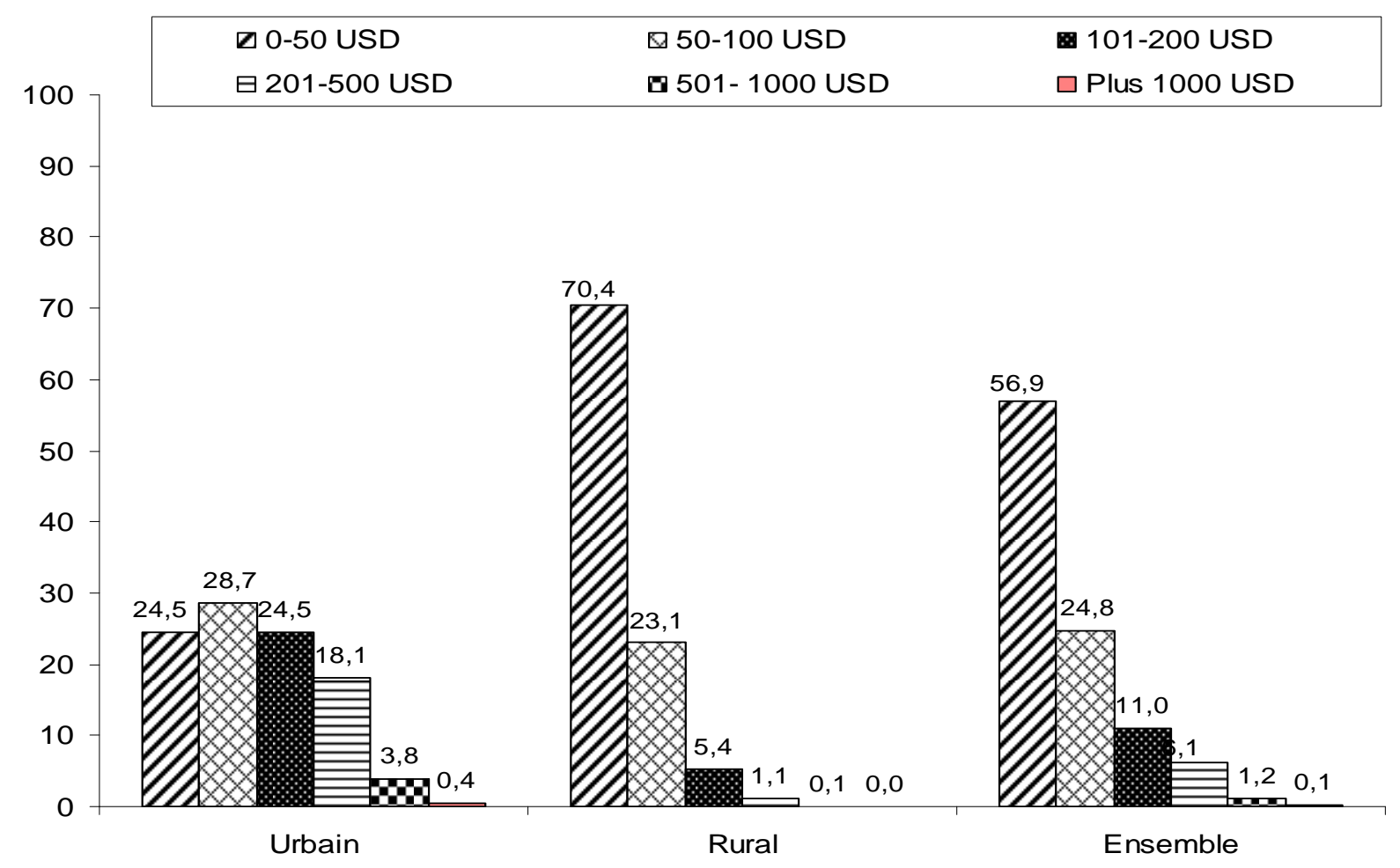

Source : Données de l'enquête auprès des ménages, EADE-RDC 2012

La comparaison des provinces en termes de distribution des classes de revenu (Graphique 15) révèle que c'est dans les provinces de l'Equateur, du Maniema et du Kasaï Oriental où il y a plus de $70 \%$ des ménages

\footnotetext{
${ }^{17}$ Toutefois soulignons que le coût de la vie est généralement plus élevé en milieu urbain qu'en milieu rural.
} 
avec un revenu mensuel inférieur à 50 S US (respectivement, 78,5\%;72,9\% et 72,2 \%). A l'opposé, Kinshasa (avec 5,2 \%), le Bas-Congo (avec 45,4\%) et le Sud-Kivu (avec 49,2 \%) sont les provinces où la proportion des ménages à très bas revenu est relativement faible (moins de $50 \%$ ). II y a une cohérence entre la classification des provinces sur la base des catégories de revenu issue de l'enquête EADE et les résultats de l'étude sur le profil de pauvreté réalisé à partir des données de l'enquête 1-2-3 de 2005 (Moummi, 2010 ) : en effet, l'étude du profil de pauvreté basée sur les données de l'enquête 1-2-3 donnait la province de l'Equateur comme celle où l'incidence de la pauvreté monétaire est la plus forte et Kinshasa, celle où elle est la plus faible ${ }^{18}$.

\section{Graphique 15 : Distribution (en \%) des classes de revenu par province}

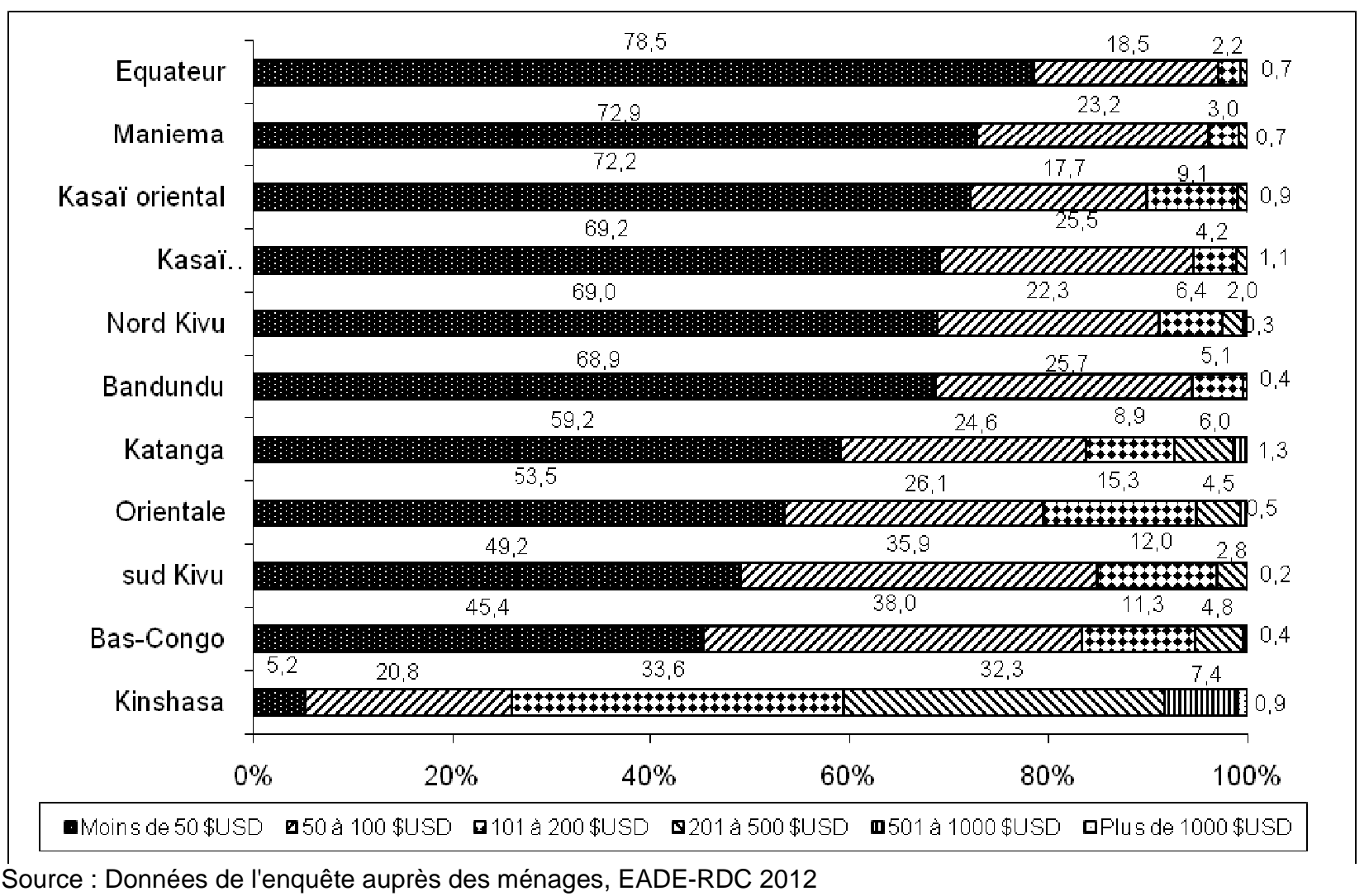

En considérant le pays dans son ensemble, il ressort que le revenu du ménage est l'une des barrières importantes à la participation scolaire des enfants et des adolescents. En effet, le revenu occupe le premier rang au niveau des 6-11 ans et le deuxième rang au niveau des 12-17 ans (tableau A29 en annexe). Cette importance du revenu comme barrière à la scolarisation des enfants est confirmée que ce soit en milieu urbain ou en milieu rural: au niveau des 6-11 ans, le revenu occupe ainsi le premier rang en milieu urbain et vient en troisième position en milieu rural. Au niveau des 12-17 ans, le revenu occupe toujours le premier rang en milieu urbain, mais par contre vient en cinquième position en milieu rural (tableau A29 en annexe).

L'examen du rôle du revenu suivant la province, montre au niveau des 6-11 ans, qu'exceptés dans la Province orientale (où le revenu vient en $7^{\text {ème }}$ position) et le Maniema (où il vient en $5^{\text {ème }}$ position), dans toutes les neuf autres provinces, le revenu du ménage est le premier ou le deuxième facteur le plus déterminant du risque de se retrouver en dehors de l'école. En considérant les 12-17 ans, le revenu du ménage fait partie des trois premières variables les plus déterminantes dans sept provinces sur les onze (Kinshasa, Bandundu, Province orientale, Nord Kivu, Sud Kivu, Katanga et Kasaï oriental).

L'examen des rapports de chances (tableau A27 en annexe) donne une idée de grandeur des écarts entre les catégories de revenu. Après contrôle du niveau d'instruction du chef de ménage et de l'ensemble des autres variables, la variable la plus discriminante en termes de risque d'être en dehors de l'école est le revenu du ménage : en effet, les rapports de chances associés aux risque d'être en dehors de l'école diminuent de manière très nette lorsqu'on passe de la deuxième classe de revenu à la quatrième classe de revenu : au niveau des 6-11 ans, un enfant dont le ménage a un revenu mensuel compris entre 50 et 100 USD a $33 \%$

\footnotetext{
${ }^{18}$ A deux rangs près, cinq (5) provinces sur les onze (11) ont le même classement sur les deux variables : profil de pauvreté (Moummi, 2010) et classe de revenu mensuel (EADE 2012).
} 
moins de risque (rapport de chances de 0,67) d'être en dehors de l'école comparé à un enfant dont le ménage a un revenu mensuel inférieur à 50 USD. Lorsque le ménage a un revenu mensuel entre 101 et 200 USD, l'enfant ou l'adolescent a $60 \%$ moins de risque d'être en dehors de l'école (rapport de chances de 0,40). Et lorsque le revenu mensuel du ménage est supérieur à 200 USD, l'enfant ou l'adolescent a $80 \%$ moins de risque d'être en dehors de l'école que celui dont le ménage a moins de 50 \$ US par mois. Quel que soit le milieu de résidence, cet effet du revenu est net, mais plus encore en milieu urbain. Comme au niveau des 611 ans, le revenu du ménage est l'une des variables les plus discriminantes au niveau des 12-17 ans, que ce soit en milieu urbain comme en milieu rural ${ }^{19}$ (tableau A29 en annexe).

Le graphique 16 montre bien comment la proportion d'EADE décroit de manière régulièrement et nette lorsque le revenu du ménage augmente : au niveau national, on passe d'une proportion d'EADE de 48,9\% dans les ménages à moins de 50 USD par mois, à 1,9\% dans les ménages à plus de 500 USD par mois. La même tendance s'observe aussi bien chez les filles que chez les garçons, avec des niveaux de proportions d'EADE plus élevées chez les filles que chez les garçons.

Graphique 16 : Proportions (en \%) des enfants et des adolescents de 5-17 ans en dehors de l'école par revenu mensuel du ménage et par sexe

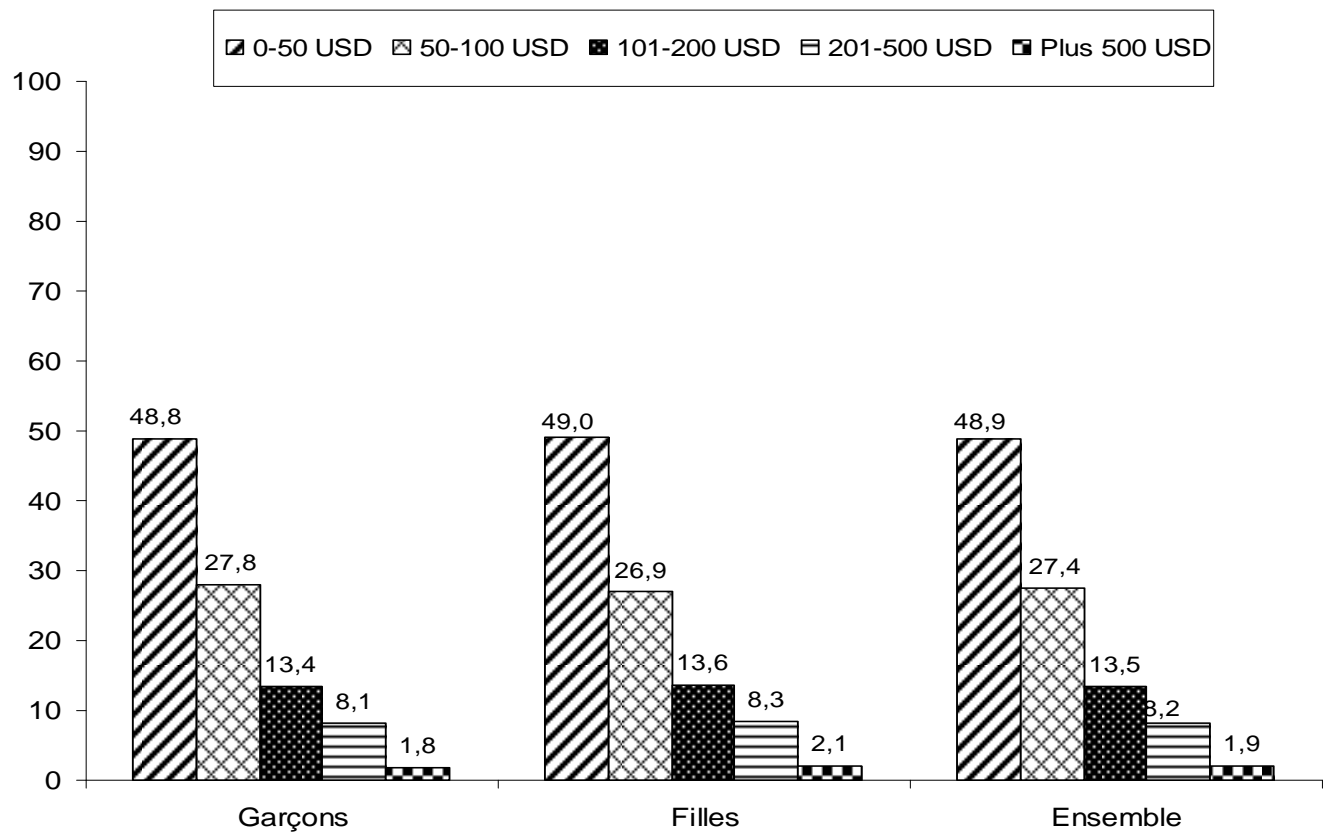

Source : Données de l'enquête auprès des ménages, EADE-RDC 2012

On peut noter également que les écarts entre ménages en fonction de la classe de revenu sont plus importants en milieu urbain qu'en milieu rural (Graphique 17).

\footnotetext{
${ }^{19}$ Les rapports de chances pour les classes de revenu supérieures en milieu rural ne sont pas statistiquement significatifs pour des raisons de faibles effectifs d'enfants dans ces catégories.
} 
Graphique 17 : Proportions (en \%) des enfants et des adolescents de 5-17 ans en dehors de l'école par revenu mensuel du ménage et par milieu de résidence

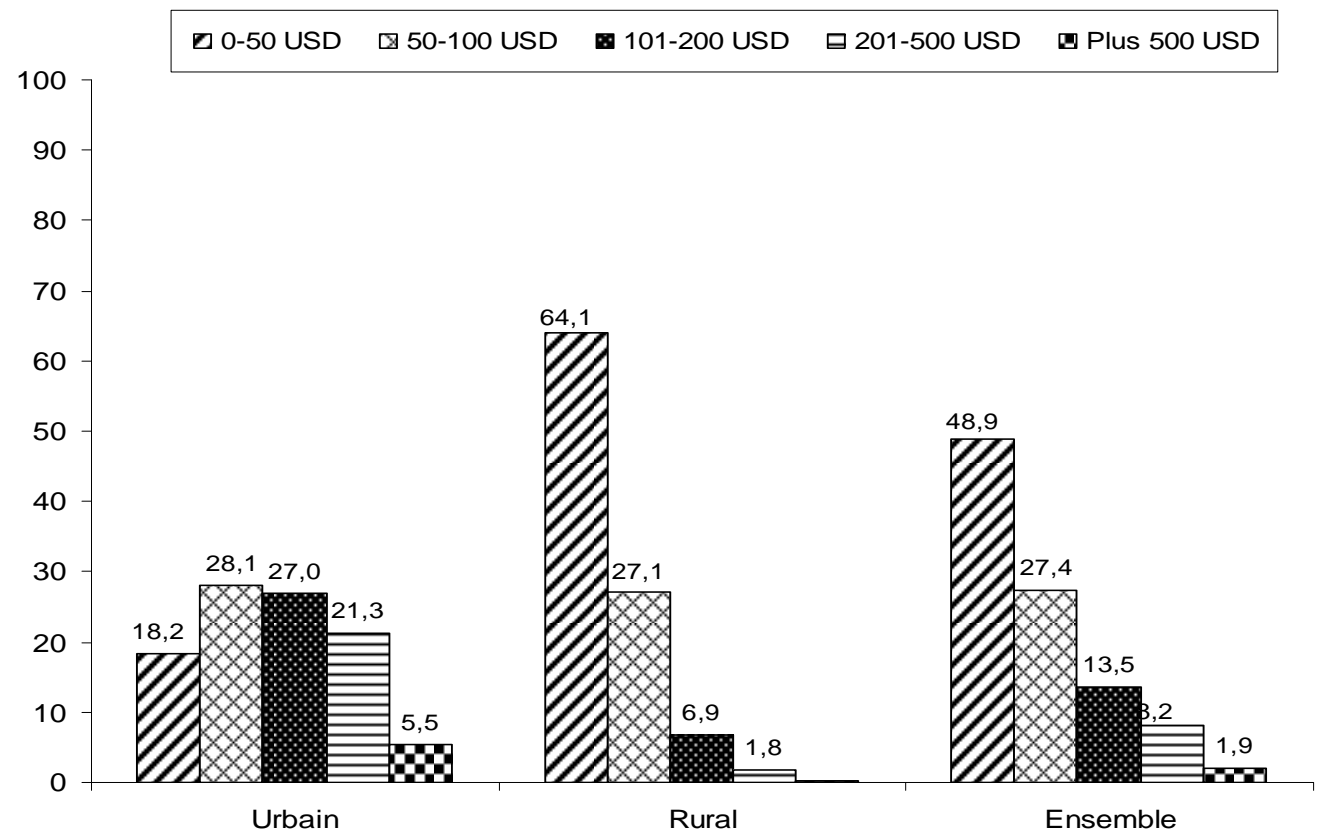

Source : Données de l'enquête auprès des ménages, EADE-RDC 2012

Cette importance de la question financière comme principale barrière à la scolarisation des enfants et des adolescents, est bien confirmée par les raisons invoquées par les ménages pour justifier la non inscription à l'école ou l'abandon scolaire. En effet, en se référant au tableau A30 en annexe, on note que quelle que soit la province, le manque d'argent vient largement en tête comme la raison la plus citée : au niveau de l'ensemble du pays, dans $69,8 \%$ des cas, elle est citée comme raison de non inscription à l'école et dans $71,0 \%$ des cas comme raison d'arrêt de l'école.

Les propos suivants issus des entretiens qualitatifs vont dans le même sens que les résultats quantitatifs et illustrent bien l'importance de la barrière financière :

"Il y a plusieurs raisons. D'abord, les raisons pécuniaires : la plupart des parents congolais sont pauvres, même ceux-là qui travaillent on est très mal payé et ce salaire vient quand l'employeur veut vous le donner, on ne sait pas s'organiser; avec ce salaire vous payez le loyer, vous payez la scolarité, la maladie, le transport tout ca; le parent dit: moi je ne peux plus supporter, choisir un ou deux enfants, les autres attendent » (Homme, cadre du système éducatif).

" [Les enfants qui ont moins de chances d'aller à l'école], ce sont les enfants qui sont nés dans des familles pauvres, c'est ça le mot clé. Quand on est pauvre, finalement on ne sait pas s'organiser à partir de la petite chose que vous avez, certainement les enfants n'auront pas la chance d'aller à l'école " (Femme, éducatrice, Nord Kivu).

"Comme nous l'avons peut-être souligné d'une manière ou d'une autre, il y a un problème de pauvreté. II manque l'argent pour payer les frais scolaires, ce que nous avons appelé prime ici. Nombreux ne vont pas à l'école à cause de cette raison. Alors il y a ce problème de pauvreté-là ». (Homme, enseignant, Nord Kivu).

Après avoir examiné l'effet du revenu sur l'exclusion scolaire, il convient à présent d'examiner ce que représentent les dépenses d'éducation des ménages congolais. L'enquête nationale sur la situation des enfants et adolescents en dehors de l'école a recueilli des informations sur les dépenses d'éducation effectuées par les ménages au cours de l'année scolaire 2010-2011. La collecte des données s'étant déroulée en 2012, le choix de l'année scolaire précédente (2010-2011) comme année de référence présentait l'avantage de pouvoir obtenir des informations plus complètes sur une année scolaire. Les enfants concernés sont ceux âgés de 3-17 ans et ayant été scolarisés en 2010-2011. Les informations ont été collectées pour 3 groupes distincts d'enfants répondant aux critères définis et présentant les profils suivants :

résident dans le ménage enquêté ;

- enfants biologiques du chef de ménage ou de sa conjointe (ou de son conjoint) vivant ailleurs ; 
- des enfants qui ne résident pas dans le ménage, ne sont pas des enfants biologiques du chef de ménage ou de sa conjointe (ou de son conjoint) mais dont le ménage enquêté a contribué à la scolarisation en 2010-2011.

Des études ont été réalisées auparavant sur la question des frais scolaires. On peut citer à ce titre l'étude intitulée "Politique et pratique des frais scolaires en RDC : frais de fonctionnement ou fonds de famille? " (Verhaghe, 2007). Mais l'enquête EADE est la première opération d'envergure à tenter d'estimer auprès des ménages, leurs dépenses d'éducation. En effet, comme le souligne le Document de stratégie de développement de l'EPSP 2010/2011-2015/2016 (RDC/MEPSP, 2010, p. 29), " il est également capital de mesurer correctement l'impact réel des contributions des parents sur le fonctionnement du système éducatif. Tout changement radical sans alternative durable pourrait avoir des conséquences néfastes. ”

Les dépenses d'éducation considérées sont catégorisées en sept postes qui ont été proposées et adoptées par les participants à l'atelier de validation des outils de collecte ${ }^{20}$ :

- les frais de scolarité et de cotisation ;

- les frais d'acquisition des fournitures scolaires ;

- les frais d'acquisition de l'uniforme ;

- les frais engagés pour l'achat de la nourriture (à l'école) ;

- l'argent de poche ;

- les frais ponctuels d'intervention ;

- les autres dépenses.

Pour l'ensemble du pays, 39,2\% de la dépense totale d'éducation sont affectés aux frais de scolarité et de cotisation (tableau 29). Le poste de dépenses relative à la nourriture de l'enfant scolarisé vient en deuxième position avec $14,8 \%$. Les postes fournitures, frais ponctuels d'intervention et uniforme ont quasiment le même poids dans les dépensent d'éducation (respectivement 10,9\%, 10,9\% et 10,8\%).

Quel que soit le milieu de résidence, le poste de dépenses prépondérant est celui des frais de scolarité et de cotisation. II représente $42,0 \%$ des dépenses d'éducation en milieu urbain et $31,3 \%$ en milieu rural. II convient de mentionner que ce soit en milieu urbain ou en milieu rural, que les dépenses de nourriture (en lien avec la scolarisation) et les frais ponctuels occupent une place importante dans les dépenses d'éducation des ménages (respectivement $14,8 \%$ et $14,8 \%$ pour les dépenses de nourriture, et $10,3 \%$ et $12,3 \%$ pour les frais ponctuels d'intervention). Trois éléments de différences apparaissent toutefois entre villes et campagnes quant à la structure des dépenses d'éducation des ménages: Les fournitures et les frais de transport représentent une part plus importante des dépenses d'éducation en milieu urbain (respectivement 15,9\% et $6,7 \%$ ) qu'en milieu rural (respectivement 9,2 \% et 0,4\%), alors que l'uniforme pèse plus dans les dépenses d'éducation en milieu rural (19,8\%) qu'en milieu urbain $(7,7 \%)$.

L'analyse selon la province révèle que le poste frais de scolarité et cotisation est le plus important dans huit des onze provinces (Sud Kivu, Kinshasa, Maniema, Kasaï occidental, Bas Congo, Equateur, Katanga et Province orientale). Dans les provinces du Nord-Kivu et du Kasaï Oriental, ce sont les frais ponctuels d'intervention qui représentent le poste plus important, avec respectivement $29,7 \%$ et $22,7 \%$ du total des dépenses d'éducation. L'uniforme vient en deuxième position dans les dépenses totales d'éducation des ménages au Kasaï Occidental (28,7\%), au Kasaï Oriental (20,5\%) et au Bandundu (24,0\%). Plus du quart $(27,6 \%)$ des dépenses totales d'éducation en 2010-2011 des ménages de la Province Orientale a été consacré à l'alimentation des enfants scolarisés.

\footnotetext{
${ }^{20}$ L'atelier de validation des outils de collecte qui a regroupé l'ensemble des parties prenantes a eu lieu du 11 au 12 août 2011. Pour la collecte des données, en plus de la formation des enquêteurs et de la présence d'un représentant de la DEP sur les frais scolaires, une note d'instruction a été élaborée à l'attention des enquêteurs pour aider à bien différencier les différents postes de dépenses liées à l'éducation.
} 
Tableau 29 : Structure (en \%) des dépenses d'éducation par poste de dépenses (en USD) des ménages pour l'année 2010-2011, par province, milieu de résidence

\begin{tabular}{|c|c|c|c|c|c|c|c|c|c|c|}
\hline Entités spatiales & $\begin{array}{l}\text { Frais scolarité } \\
\text { et cotisation }\end{array}$ & Fournitures & Transport & Uniforme & Nourriture & $\begin{array}{l}\text { Argent de } \\
\text { poche }\end{array}$ & $\begin{array}{c}\text { Frais } \\
\text { ponctuels } \\
\text { d'intervention }\end{array}$ & $\begin{array}{c}\text { Autres } \\
\text { dépenses }\end{array}$ & Total & $\begin{array}{c}\text { Somme des } \\
\text { dépenses totales } \\
\text { pour la scolarisation } \\
\text { en } 2010-2011\end{array}$ \\
\hline \multicolumn{11}{|l|}{ Provinces } \\
\hline Kinshasa & 45,8 & 7,9 & 8,0 & 5,7 & 13,9 & 9,6 & 8,0 & 1,1 & 100,0 & 628101541,8 \\
\hline Bas-Congo & 37,3 & 14,5 & 5,4 & 13,0 & 13,4 & 11,4 & 3,2 & 1,8 & 100,0 & 62440669,5 \\
\hline Bandundu & 28,0 & 18,0 & 1,9 & 24,0 & 9,5 & 10,1 & 6,8 & 1,6 & 100,0 & 53288246,5 \\
\hline Equateur & 34,3 & 19,0 & 0,9 & 18,2 & 8,0 & 5,7 & 13,0 & 0,9 & 100,0 & 51083272,2 \\
\hline Orientale & 30,0 & 11,4 & 0,6 & 16,2 & 27,6 & 4,1 & 6,7 & 3,3 & 100,0 & 122801350,4 \\
\hline Nord Kivu & 29,3 & 10,3 & 1,5 & 15,9 & 12,5 & 0,4 & 29,7 & 0,5 & 100,0 & 71277865,9 \\
\hline Maniema & 46,1 & 23,5 & 2,1 & 19,4 & 0,7 & 2,3 & 5,7 & 0,3 & 100,0 & 12251242,4 \\
\hline sud Kivu & 53,4 & 16,7 & 1,0 & 18,3 & 2,0 & 1,5 & 6,2 & 1,0 & 100,0 & 44164426,0 \\
\hline Katanga & 33,6 & 10,7 & 4,2 & 9,6 & 19,2 & 4,7 & 17,4 & 0,8 & 100,0 & 233050420,1 \\
\hline Kasaï oriental & 21,4 & 16,7 & 0,7 & 20,5 & 12,1 & 4,6 & 22,7 & 1,4 & 100,0 & 39726135,4 \\
\hline Kasaï occidental & 38,8 & 22,5 & 0,3 & 28,7 & 2,0 & 2,5 & 4,1 & 1,0 & 100,0 & 26217848,9 \\
\hline \multicolumn{11}{|c|}{ Milieu de résidence } \\
\hline Urbain & 42,0 & 9,2 & 6,7 & 7,7 & 14,8 & 8,3 & 10,3 & 1,0 & 100,0 & 996227627,1 \\
\hline Rural & 31,3 & 15,9 & 0,4 & 19,8 & 14,8 & 3,6 & 12,3 & 1,9 & 100,0 & 348175391,8 \\
\hline Ensemble RDC & 39,2 & 10,9 & 5,0 & 10,8 & 14,8 & 7,1 & 10,9 & 1,3 & 100,0 & 1344403018,9 \\
\hline
\end{tabular}

Source : Données de l'enquête auprès des ménages, EADE-RDC 2012 
En s'intéressant aux dépenses moyennes de scolarisation des enfants en 2010-2011, il ressort du tableau 30 qu'un ménage en RD Congo a dépensé en moyenne 201,3 dollars américains pour la scolarisation de ses enfants (tableau 30). Cette dépense moyenne était plus importante en milieu urbain (430,3 USD) qu'en milieu rural (79,8 USD). Elle était également plus importante dans les trois provinces abritant les trois principales villes du pays, notamment à Kinshasa (677,9 USD), au Katanga (228,1 USD) et dans la Province Orientale (172,9 UDS). Elle était par contre plus faible au Kasaï Occidental (63,0 USD), dans le Bandundu (63,8 \%), au Kasaï Oriental (71,2 USD), dans le Maniema $(74,2$ USD) et à l'Equateur (73,6 USD).

Un intérêt de l'analyse des dépenses d'éducation est de voir ce que celles-ci représentent dans le revenu des ménages. Pour estimer ce revenu annuel, nous avons considéré comme revenu mensuel du ménage le centre de classe du revenu mensuel. Ainsi pour les ménages dont le revenu est inférieur à 50 USD, il leur a été affecté 25 USD, pour la classe de revenu $50-100$ USD, la valeur 75, pour la classe $101-200$ USD, la valeur 150 USD, pour la classe $201-500$ USD, la valeur 350 USD, pour la classe $501-1000$ USD, la valeur 750 et enfin pour les ménages ayant plus de 1000 USD, la valeur 1250 (en supposant que la borne supérieure de cette classe de revenu est de 1500 USD). Ensuite, pour avoir un revenu annuel pour chaque ménage, cette valeur mensuelle a été multipliée par 12.

Tableau 30 : Dépense moyenne d'éducation (en USD) des ménages pour l'année scolaire 2010-2011, par entité spatiale de résidence

\begin{tabular}{|c|c|c|c|c|c|c|}
\hline \multirow{3}{*}{ Entités spatiales } & \multicolumn{4}{|c|}{ Dépenses moyennes de scolarisation en 2010-2011 } & \multirow{2}{*}{\multicolumn{2}{|c|}{$\begin{array}{c}\text { Dépenses totales pour la } \\
\text { scolarisation en 2010-2011 }\end{array}$}} \\
\hline & \multicolumn{2}{|c|}{ Enfants du ménage } & \multirow{2}{*}{$\begin{array}{l}\text { Autres enfants } \\
\text { à la charge du } \\
\text { ménage }\end{array}$} & \multirow{2}{*}{$\begin{array}{l}\text { Tous les } \\
\text { enfants } \\
\text { scolarisés }\end{array}$} & & \\
\hline & $\begin{array}{l}\text { Dans le } \\
\text { ménage }\end{array}$ & $\begin{array}{l}\text { En dehors du } \\
\text { ménage }\end{array}$ & & & Somme & $\begin{array}{l}\text { Ecart- } \\
\text { type }\end{array}$ \\
\hline \multicolumn{7}{|l|}{ Provinces } \\
\hline Kinshasa & 637,5 & 25,9 & 14,4 & 677,9 & 628101541,8 & 707,7 \\
\hline Bas-Congo & 124,1 & 13,6 & 2,7 & 140,5 & 62440669,5 & 225,4 \\
\hline Bandundu & 53,4 & 6,7 & 3,7 & 63,8 & 53288246,5 & 105,0 \\
\hline Equateur & 67,1 & 4,3 & 2,3 & 73,6 & 51083272,2 & 92,5 \\
\hline Orientale & 145,6 & 16,9 & 10,4 & 172,9 & 122801350,4 & 278,0 \\
\hline Nord Kivu & 135,2 & 5,4 & 9,0 & 149,6 & 71277865,9 & 199,5 \\
\hline Maniema & 65,5 & 3,6 & 5,2 & 74,2 & 12251242,4 & 93,3 \\
\hline sud Kivu & 87,4 & 8,9 & 6,4 & 102,7 & 44164426,0 & 128,7 \\
\hline Katanga & 214,0 & 9,1 & 4,9 & 228,1 & 233050420,1 & 387,3 \\
\hline Kasaï oriental & 62,6 & 5,4 & 3,3 & 71,2 & 39726135,4 & 106,7 \\
\hline Kasaï occidental & 50,3 & 7,1 & 5,6 & 63,0 & 26217848,9 & 76,7 \\
\hline \multicolumn{7}{|l|}{ Milieu de résidence } \\
\hline Urbain & 402,2 & 16,9 & 11,2 & 430,3 & 996227627,1 & 574,0 \\
\hline Rural & 68,0 & 7,7 & 4,1 & 79,8 & 348175391,8 & 120,8 \\
\hline Ensemble RDC & 183,8 & 10,9 & 6,5 & 201,3 & 1344403018,9 & 389,3 \\
\hline
\end{tabular}

Source : Données de l'enquête auprès des ménages, EADE-RDC 2012

Un ménage en RD Congo a consacré en 2010-2011 plus du dixième (11,2\%) de son revenu annuel aux dépenses d'éducation des enfants. Les ménages des provinces de Kinshasa (15,5\%), du Katanga $(11,7 \%)$ et du Nord-Kivu $(11,9 \%)$ sont ceux qui ont consacré une part relativement importante de leur revenu annuel aux dépenses d'éducation (tableau 31). La part du revenu annuel consacrée aux dépenses d'éducation des enfants en 2010-2011 en milieu urbain (14,1\%) était le double de celle observée en milieu rural $(7,0 \%)$. En plus de ceux de Kinshasa, les ménages urbains du Nord-Kivu (20,8\%) et du Katanga (15,0\%) sont ceux qui ont une part relativement importante des dépenses d'éducation dans le revenu annuel.

En mettant cette fois-ci les dépenses d'éducation des ménages de chaque catégorie sociale avec une estimation de leur revenu moyen annuel, il ressort que la part des dépenses d'éducation dans le revenu annuel estimé des différentes catégories sociales est quasiment constante d'une classe à l'autre : cette part est de 11,3\% pour les ménages de moins de 50 USD par mois, de 9,7\% pour les ménages ayant entre 50 et 100 USD, de $11,7 \%$ pour les ménages dont le revenu mensuel va de 101 USD à 200 USD et de 11,6\% pour les ménages de revenu supérieur à 200 USD par mois. 
Tableau 31 : Part des dépenses d'éducation dans le revenu annuel estimé des ménages (en USD) pour l'année 2010-2011, par milieu de résidence

\begin{tabular}{|c|c|c|c|c|c|c|c|c|c|}
\hline \multirow[b]{2}{*}{ Provinces } & \multicolumn{3}{|c|}{ Urbain } & \multicolumn{3}{|c|}{ Rural } & \multicolumn{3}{|c|}{ Ensemble RDC } \\
\hline & $\begin{array}{c}\text { Dépenses } \\
\text { totales des } \\
\text { ménages pour } \\
\text { l'éducation en } \\
2010-2011\end{array}$ & $\begin{array}{l}\text { Revenu annuel } \\
\text { moyen des } \\
\text { ménages }\end{array}$ & $\begin{array}{c}\text { Part des } \\
\text { dépenses } \\
\text { d'éducation }\end{array}$ & $\begin{array}{c}\text { Dépenses } \\
\text { totales des } \\
\text { ménages pour } \\
\text { l'éducation en } \\
2010-2011\end{array}$ & $\begin{array}{l}\text { Revenu annuel } \\
\text { moyen des } \\
\text { ménages }\end{array}$ & $\begin{array}{l}\text { Part des } \\
\text { dépenses } \\
\text { d'éducation }\end{array}$ & $\begin{array}{l}\text { Dépenses totales } \\
\text { des ménages pour } \\
\text { l'éducation en } \\
2010-2011\end{array}$ & $\begin{array}{l}\text { Revenu annuel } \\
\text { moyen des } \\
\text { ménages }\end{array}$ & $\begin{array}{l}\text { Part des } \\
\text { dépenses } \\
\text { d'éducatio }\end{array}$ \\
\hline Kinshasa & 628101541,8 & 4058579885,5 & 15,5 & - & - & - & 628101541,8 & 4058579885,5 & 15,5 \\
\hline Bas-Congo & 29079526,0 & 294853628,5 & 9,9 & 33361143,4 & 470262 679,0 & 7,1 & 62440 669,5 & 765116307,5 & 8,2 \\
\hline Bandundu & 16135687,7 & 167881716,8 & 9,6 & 37152558,8 & 580752861,1 & 6,4 & 53288246,5 & 748634577,9 & 7,1 \\
\hline Equateur & 17371543,9 & 156196005,1 & 11,1 & 33711728,3 & 488546083,7 & 6,9 & 51083272,2 & 644742088,8 & 7,9 \\
\hline Orientale & 37413357,8 & 376000678,6 & 10,0 & 85387992,6 & 988142634,3 & 8,6 & 122801350,4 & 1364143312,8 & 9,0 \\
\hline Nord Kivu & 43546660,9 & 209820731,3 & 20,8 & 27731205,0 & 389866551,6 & 7,1 & 71277865,9 & 599687282,9 & 11,9 \\
\hline Maniema & 5222 416,8 & 51985 199,8 & 10,0 & 7028825,6 & 119659707,5 & 5,9 & 12251242,4 & 171644907,3 & 7,1 \\
\hline sud Kivu & 14224455,0 & 187412312,1 & 7,6 & 29939971,0 & 496186513,8 & 6,0 & 44164426,0 & 683598825,9 & 6,5 \\
\hline Katanga & 178558845,6 & 1192808146,5 & 15,0 & 54491574,4 & 798955875,4 & 6,8 & 233050420,1 & 1991764021,9 & 11,7 \\
\hline Kasaï oriental & 22058529,0 & 326110063,1 & 6,8 & 17667606,4 & 269567411,4 & 6,6 & 39726135,4 & 595677474,5 & 6,7 \\
\hline Kasaï occidental & 4515062,6 & 59523616,3 & 7,6 & 21702786,4 & 366061831,5 & 5,9 & 26217848,9 & 425585447,8 & 6,2 \\
\hline Total & 996227627,1 & 7081171983,6 & 14,1 & 348175391,8 & $4968002 \quad 149,2$ & 7,0 & 1344403018,9 & 12049174 132,8 & 11,2 \\
\hline
\end{tabular}

Source : Données de l'enquête auprès des ménages, EADE-RDC 2012 


\section{b) Travail des enfants}

L'effet du travail des enfants sur leur participation à l'école n'est pas aisé à mettre en évidence d'un point de vue quantitatif, dans la mesure où ces deux activités de l'enfant ne sont pas nécessairement indépendantes ${ }^{21}$. Une manière de procéder est d'examiner d'une part la proportion des EADE qui travaillent et d'autre part, examiner la proportion des enfants au travail qui sont EADE. Mais l'ampleur de cette double facette de l'interaction entre participation au travail et participation à l'école dépend énormément de la définition du travail. Est-ce le travail considéré au sens large (aussi bien travail économique que travail non économique) ou le travail au sens économique et au sens de l'UCW, prenant en compte le nombre d'heures de travail ? Le graphique 18 illustre assez bien cela : en considérant le travail au sens large, on note que les EADE au travail représentent 3,7\% des enfants de 6-13 ans ${ }^{22}$ et les EADE non au travail 0,2\%. Les élèves de 6-13 ans au travail représentent $88,9 \%$ tandis que les élèves non au travail représentent $7,1 \%$ des $6-13$ ans. Autre lecture à travers ce graphique : au sein des EADE qui représentent $4,0 \%$ des 6-13 ans, 92,5\% sont au travail, tandis que parmi les enfants de $6-13$ ans au travail (représentant 92,6\% des 6-13 ans), 4,0\% sont des EADE. En d'autres termes plusieurs enfants d'âges scolaires combinent participation scolaire et exercice d'un travail, alors qu'une large partie des enfants en dehors de l'école sont davantage impliqués dans le travail.

Graphique 18 : Profil scolaire et implication dans le travail des enfants et des adolescents de 6-13 ans en fonction de la définition du travail

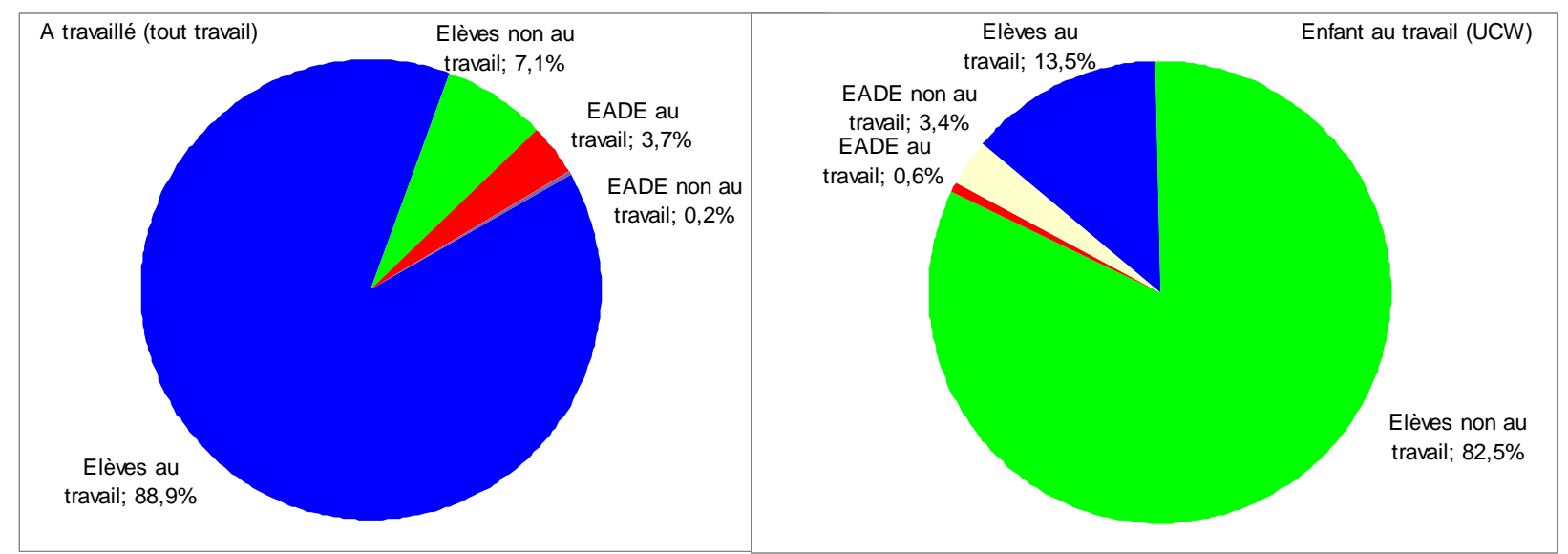

Source : Données de l'enquête auprès des ménages, EADE-RDC 2012

Lorsqu'on considère la définition du travail au sens UCW, les résultats changent considérablement (graphique 18). Dans la suite de nos analyses et pour tenir compte des recommandations internationales, c'est le travail au sens UCW qui sera privilégié.

\subsubsection{Enfants en dehors de l'école et impliqués dans le travail}

En considérant la définition du travail au sens UCW, il ressort pour l'ensemble de la RDC que $14,4 \%$ des EADE de 6-13 ans travaillaient au moment de l'enquête en 2012 (tableau 32). Cette participation des EADE au travail croît rapidement avec l'âge. La participation des filles EADE au travail est plus forte que celles des garçons EADE à certains âges. La participation au travail des EADE est très variable selon le milieu avec des différences significatives entre filles EADE du milieu urbain $(17,7 \%)$ et celles du milieu rural $(3,1 \%)$. A l'inverse, les filles du milieu rural participent au travail plus que les garçons du milieu rural (respectivement $28,3 \%$ et $13,4 \%$ ).

Quelle que soit la province, on trouve des EADE impliqués dans le travail. Les provinces du Bandundu, du Katanga et du Bas-Congo se distinguent par une proportion nettement plus importante d'EADE exerçant un travail (plus de un EADE sur cinq).

\footnotetext{
${ }^{21}$ Du fait de l'endogénéité de la décision de travailler et d'aller à l'école, il est difficile, surtout avec des données transversales d'établir des liens de causalité entre le fait de travailler et d'aller à l'école.

${ }^{22}$ Ce groupe d'âges est celui proposé dans la méthodologie de UCW.
} 
Tableau 32 : Proportions (en \%) des EADE d'âges du primaire et du secondaire en dehors de l'école impliqués dans le travail selon les caractéristiques de l'enfant et de leur ménage

\begin{tabular}{|c|c|c|c|c|c|c|}
\hline \multirow{2}{*}{ Caractéristiques } & \multicolumn{3}{|c|}{ Proportions } & \multicolumn{3}{|c|}{ Effectifs de travailleurs } \\
\hline & Garçons & Filles & Ensemble & Garçons & Filles & Ensemble \\
\hline \multicolumn{7}{|l|}{ Age } \\
\hline 6 & 22,8 & - & 6,6 & 1366 & - & 1366 \\
\hline 7 & 4,1 & 6,4 & 5,0 & 1310 & 1414 & 2724 \\
\hline 8 & 10,4 & 7,5 & 8,9 & 2927 & 2108 & 5035 \\
\hline 9 & 7,4 & 11,3 & 9,7 & 1697 & 3673 & 5370 \\
\hline 10 & 17,0 & 18,4 & 17,7 & 6409 & 6597 & 13006 \\
\hline 11 & 18,3 & 12,1 & 14,2 & 2979 & 3746 & 6725 \\
\hline 12 & 22,7 & 16,6 & 19,4 & 7012 & 6014 & 13026 \\
\hline 13 & 24,8 & 32,2 & 27,9 & 7171 & 6840 & 14011 \\
\hline \multicolumn{7}{|l|}{ Milieu de résidence } \\
\hline Urbain & 17,7 & 3,1 & 9,6 & 15171 & 3413 & 18584 \\
\hline Rural & 13,4 & 23,8 & 18,5 & 15700 & 26979 & 42679 \\
\hline \multicolumn{7}{|l|}{ Province } \\
\hline Kinshasa & 14,2 & - & 6,3 & 4668 & - & 4668 \\
\hline Bas-Congo & 18,4 & 24,3 & 22,5 & 1015 & 2931 & 3946 \\
\hline Bandundu & 39,9 & 36,4 & 37,5 & 1933 & 3721 & 5654 \\
\hline Equateur & 0,0 & 19,6 & 10,7 & - & 4517 & 4517 \\
\hline Orientale & 12,2 & 7,7 & 10,7 & 4323 & 1414 & 5737 \\
\hline Nord Kivu & 10,2 & 22,8 & 14,8 & 2537 & 3250 & 5787 \\
\hline Maniema & 0,0 & 24,5 & 12,0 & - & 893 & 893 \\
\hline sud Kivu & 12,9 & 23,2 & 18,2 & 1618 & 3072 & 4690 \\
\hline Katanga & 33,9 & 19,7 & 26,8 & 14778 & 8645 & 23423 \\
\hline Kasaï oriental & & 1,8 & 1,2 & - & 484 & 484 \\
\hline Kasaï occidental & - & 10,2 & 6,4 & - & 1464 & 1464 \\
\hline \multicolumn{7}{|l|}{ Revenu mensuel } \\
\hline Moins de 50 dollars & 11,4 & 18,4 & 15,1 & 10891 & 20832 & 31723 \\
\hline $50-100$ dollars & 19,7 & 11,5 & 16,0 & 11457 & 5547 & 17004 \\
\hline 101-200 dollars & 20,6 & 4,7 & 11,9 & 6955 & 1945 & 8900 \\
\hline Plus de 200 dollars & $100,0^{23}$ & 11,4 & 10,7 & 1569 & 2067 & 3636 \\
\hline \multicolumn{7}{|c|}{ Sexe du chef de ménage } \\
\hline Hommes & 17,0 & 16,3 & 16,7 & 21725 & 21311 & 43036 \\
\hline Femmes & 12,2 & 9,9 & 10,9 & 9147 & 9080 & 18227 \\
\hline \multicolumn{7}{|c|}{ Niveau d'instruction du chef de ménage } \\
\hline Aucun & 17,5 & 14,0 & 15,7 & 21143 & 18321 & 39464 \\
\hline Primaire & 15,7 & 22,2 & 18,6 & 6093 & 7103 & 13196 \\
\hline Secondaire et plus & 8,4 & 8,4 & 8,4 & 3636 & 4967 & 8603 \\
\hline Ensemble RDC & 15,2 & 13,7 & 14,4 & 30872 & 30391 & 61263 \\
\hline
\end{tabular}

Source : Données de l'enquête auprès des ménages, EADE-RDC 2012 Effectifs très faibles

Lorsqu'on tient compte du niveau de revenu des ménages, c'est dans les ménages à revenu inférieur à 50 USD que les garçons EADE sont plus impliqués au travail. Les filles EADE le sont aussi, mais dans une moindre mesure. Avec les chefs de ménages hommes, l'implication au travail des garçons et des filles EADE est presque dans les mêmes proportions (tableau 32). Avec les chefs de ménages femmes, l'implication au travail des EADE est plus important pour les garçons. On relève aussi que plus d'une fille EADE sur cinq est impliquée dans le travail chez les chefs de ménage de niveau d'instruction primaire.

\footnotetext{
${ }^{23}$ Du fait des faibles effectifs d'EADE dans ce groupe, tous semblent être impliqués au travail. D'où cette proportion de $100 \%$.
} 
En regardant cette fois-ci la situation des enfants au travail en termes de participation scolaire (tableau 33), il ressort pour l'ensemble du pays, que parmi les 6-13 ans au travail au sens UCW, 4,0\% \% sont en dehors de l'école. Un résultat à souligner est le fait que la proportion d'enfants au travail et qui sont à l'école, se maintient tout au long de la scolarité montrant ainsi le fait que les élèves combinent travail et scolarisation.

La proportion d'enfants au travail en dehors de l'école n'est pas très différente chez les filles et les garçons (respectivement $4,4 \%$ et $3,8 \%$ ). Mais cette différence est assez variable selon l'âge des enfants.

Tableau 33 : Proportions (en \%) d'enfants au travail d'âges du primaire et du secondaire impliqués statut scolaire, âge et sexe de l'enfant

\begin{tabular}{|c|c|c|c|c|c|c|c|c|c|}
\hline \multirow{2}{*}{ Statut scolaire } & \multicolumn{8}{|c|}{ Age } & \multirow{2}{*}{ Ensemble } \\
\hline & 6 & 7 & 8 & 9 & 10 & 11 & 12 & 13 & \\
\hline \multicolumn{10}{|l|}{ Garçons } \\
\hline Scolarisé & 91,5 & 96,9 & 95,5 & 98,1 & 94,6 & 97,6 & 96,7 & 95,1 & 96,2 \\
\hline EADE & 8,5 & 3,1 & 4,5 & 1,9 & 5,4 & 2,4 & 3,3 & 4,9 & 3,8 \\
\hline Total & 100,0 & 100,0 & 100,0 & 100,0 & 100,0 & 100,0 & 100,0 & 100,0 & 100,0 \\
\hline Effectifs & 16016 & 41778 & 64666 & 87146 & 119128 & 125594 & 213158 & 145473 & 812959 \\
\hline \multicolumn{10}{|l|}{ Filles } \\
\hline Scolarisé & 100,0 & 93,8 & 96,9 & 94,4 & 94,4 & 97,0 & 95,8 & 95,1 & 95,6 \\
\hline EADE & 0,0 & 6,2 & 3,1 & 5,6 & 5,6 & 3,0 & 4,2 & 4,9 & 4,4 \\
\hline Total & 100,0 & 100,0 & 100,0 & 100,0 & 100,0 & 100,0 & 100,0 & 100,0 & 100,0 \\
\hline Effectifs & 8106 & 22960 & 68687 & 65339 & 117047 & 126715 & 143293 & 138805 & 690952 \\
\hline \multicolumn{10}{|l|}{ Ensemble } \\
\hline Scolarisé & 94,3 & 95,8 & 96,2 & 96,5 & 94,5 & 97,3 & 96,3 & 95,1 & 95,9 \\
\hline EADE & 5,7 & 4,2 & 3,8 & 3,5 & 5,5 & 2,7 & 3,6 & 4,9 & 4,0 \\
\hline Total & 100,0 & 100,0 & 100,0 & 100,0 & 100,0 & 100,0 & 100,0 & 100,0 & 100,0 \\
\hline Effectifs & 24122 & 64738 & 133353 & 152485 & 236175 & 252309 & 356451 & 284278 & 1503911 \\
\hline
\end{tabular}

Source : Données de l'enquête auprès des ménages, EADE-RDC 2012

Toujours sur le registre de la situation des enfants au travail en termes de participation scolaire (tableau 34), la participation au travail des EADE est proportionnellement plus importante en milieu urbain. Dans toutes les provinces, les enfants impliqués dans le travail sont en majorité des élèves. II en est de même lorsqu'on examine la situation des enfants au travail en termes de participation scolaire suivant le revenu mensuel du ménage, le sexe du chef de ménage et le niveau d'instruction du chef de ménage. 
Tableau 34 : Proportions (en \%) d'enfants d'âges du primaire et du secondaire impliqués dans le travail par statut scolaire selon certaines caractéristiques du ménage

\begin{tabular}{|c|c|c|c|c|c|}
\hline \multirow[b]{2}{*}{ Caractéristiques } & \multicolumn{4}{|c|}{ Statut scolaire } & \multirow[b]{2}{*}{ Effectifs } \\
\hline & Scolarisé & Déscolarisé & $\begin{array}{c}\text { Jamais } \\
\text { fréquenté }\end{array}$ & Total & \\
\hline \multicolumn{6}{|l|}{ Milieu } \\
\hline Urbain & 93,9 & 5,5 & 0,6 & 100,0 & 303796 \\
\hline Rural & 96,4 & 3,6 & 0,0 & 100,0 & 1200117 \\
\hline \multicolumn{6}{|l|}{ Provinces } \\
\hline Kinshasa & 93,6 & 4,4 & 2,0 & 100,0 & 73151 \\
\hline Bas-Congo & 97,6 & 2,4 & 0,0 & 100,0 & 166499 \\
\hline Bandundu & 97,5 & 2,5 & 0,0 & 100,0 & 229391 \\
\hline Equateur & 96,4 & 3,6 & 0,0 & 100,0 & 127152 \\
\hline Orientale & 96,9 & 3,1 & 0,0 & 100,0 & 186691 \\
\hline Nord Kivu & 91,4 & 8,6 & 0,0 & 100,0 & 67407 \\
\hline Maniema & 98,6 & 0,7 & 0,7 & 100,0 & 65840 \\
\hline sud Kivu & 96,9 & 3,1 & 0,0 & 100,0 & 151999 \\
\hline Katanga & 90,6 & 9,4 & 0,0 & 100,0 & 248569 \\
\hline Kasaï oriental & 99,4 & 0,6 & 0,0 & 100,0 & 87881 \\
\hline Kasaï occidental & 98,5 & 1,5 & 0,0 & 100,0 & 99333 \\
\hline \multicolumn{6}{|l|}{ Revenu mensuel } \\
\hline Moins de 50 dollars & 95,9 & 4,1 & - & 100,0 & 777757 \\
\hline 50-100 dollars & 96,2 & 3,8 & - & 100,0 & 453283 \\
\hline 101-200 dollars & 94,9 & 4,2 & 0,8 & 100,0 & 175705 \\
\hline Plus de 200 dollars & 96,3 & 3,3 & 0,4 & 100,0 & 97168 \\
\hline \multicolumn{6}{|l|}{ Sexe du CM } \\
\hline Hommes & 95,2 & 4,7 & - & 100,0 & 905336 \\
\hline Femmes & 97,0 & 2,8 & 0,2 & 100,0 & 598576 \\
\hline \multicolumn{6}{|c|}{ Niveau d'instruction du CM } \\
\hline Aucun & 95,3 & 4,5 & 0,2 & 100,0 & 840833 \\
\hline Primaire & 93,4 & 6,6 & - & 100,0 & 199068 \\
\hline Secondaire et plus & 98,1 & 1,9 & - & 100,0 & 464031 \\
\hline
\end{tabular}

Source : Données de l'enquête auprès des ménages, EADE-RDC 2012 Effectifs très faibles

Si l'effet du travail sur l'exclusion scolaire n'est pas aisé à mettre en évidence du point de vue statistique (à travers les analyses mutivariées réalisées), les résultats des entretiens qualitatifs mettent bien en évidence les conséquences de cette implication des enfants dans le travail sur leur participation scolaire :

"D'une manière générale, ils [les enfants travailleurs] n'ont pas cette chance là parce que c'est difficile de combiner les activités scolaires et le travail. Bien sûr, il y a certains enfants qui se débrouillent, qui étudient, ils font le petit commerce, vendre le pétrole à la maison, il y a d'autres qui sont photographes, il y a d'autres qui travaillent dans des salons de coiffure. Un peu après ils trouvent l'argent. Pour ceux-là qui se débrouillent, cela leur permet de payer les frais scolaires par rapport aux autres. Mais du point de vue réussite, il y a ceux-là aussi qui, en travaillant n'arrivent pas à étudier leurs leçons, ils n'ont pas de temps de pouvoir étudier leurs leçons. Ils viennent juste pour accomplir la formalité de se présenter à l'école mais ils ne réussissent pas (Homme, enseignant, Nord Kivu).

"Leur accès à l'éducation pour ces enfants [enfants travailleurs], ce n'est pas tellement bien...Parce que vous savez, quant l'enfant n'a pas le temps de réviser ses notes de cours, ni de faire ses devoirs... vous voyez qu'il est d'abord quelque part occupé [au travail], s'époumoner pour l'école... vraiment c'est très différent de l'enfant qui est chez lui, qui n'est pas travailleur. Donc, celui-là a au moins ses notes, a tout moment il a quand même le temps de revoir ces notes. Mais un travailleur, il peut être fatigué, il vient du travail, tout fatigué ... il ne saura pas suivre les cours comme il faut .Vous voyez que ça crée toujours les anomalies. »(Femme, parent d'enfant, Kinshasa). 


\subsubsection{Déterminants ou barrières relevant de l'offre}

\section{a) Disponibilité et répartition des infrastructures}

L'offre scolaire s'entend ici les infrastructures scolaires, les équipements et le personnel enseignant aux trois niveaux d'enseignement. L'infrastructure scolaire est analysée de façon globale. II s'agit d'apprécier la «normalité » de l'école en termes de nombre de classes dont elle dispose.

\section{Le pré-primaire}

Le nombre d'écoles maternelles est passé de 2428 en 2006-2007 à 3396 en 2010-2011, soit une augmentation de 39,9\% au cours des cinq années (968 en termes absolus). Cette forte augmentation de l'offre en infrastructures du pré-primaire a été surtout nette dans la ville province de Kinshasa (tableau 35). Sur l'ensemble des 3396 écoles maternelles en 2011, une grande part (35,1\%) se trouve dans la ville province de Kinshasa, ce qui montre bien que le pré-primaire reste encore une réalité essentiellement urbaine. Après Kinshasa, viennent les provinces du Katanga (avec 13,7 \% des infrastructures), le Kasaï oriental (10,1\%) et le Bandundu (10,0 \%). Le Maniema, le Sud Kivu, le Nord Kivu et le Kasaï occidental, sont moins bien lotis en écoles maternelles.

On trouve en général des écoles à trois classes (ratio classes/écoles de 3). Le nombre moyen d'élèves par classe est de 25. Dans certaines provinces telles que le Sud-Kivu, le Katanga, le Nord-Kivu et le Kasaï oriental, le ratio élèves par classe est plus de 25 (respectivement 30, 29, 28 et 28).

\section{Le primaire}

Si en termes relatifs, l'accroissement du nombre d'écoles primaires $(28,3 \%)$ a été plus faible que celui des maternelles $(39,9 \%)$, en termes absolus cependant, c'est là qu'on observe la plus forte croissance de l'offre éducative : le nombre d'écoles primaires est passé de 29420 en 2006-2007 à 37749 en 2010-2011, soit 8329 écoles de plus en cinq ans (tableau 35). Sur l'ensemble des écoles primaires de la RDC en 2011, cinq provinces en concentrent plus de la moitié (61,3\%) : il s'agit du Bandundu et du Kasaï occidental (12,8\% chacun), de l'Equateur $(12,6)$, de la Province orientale $(11,4 \%)$, et du Katanga $(11,7$ $\%)$.

Il y a en moyenne 39 élèves par classe. On note cependant que dans certaines provinces les classes sont moins chargées que dans d'autres. C'est le cas de la province du Bandundu avec une moyenne par classe de 31 élèves, alors que la province du Katanga domine avec 44 élèves par classe en moyenne (tableau 36). 
Tableau 35 : Répartition (en \%) des écoles du pré-primaire, du primaire et du secondaire par province de 2006 à 2011

\begin{tabular}{|c|c|c|c|c|c|c|c|c|c|c|c|c|c|c|c|}
\hline \multirow[b]{2}{*}{ Provinces } & \multicolumn{5}{|c|}{ Ecoles pré-primaires } & \multicolumn{5}{|c|}{ Ecoles primaires } & \multicolumn{5}{|c|}{ Ecoles secondaires } \\
\hline & 2006-07 & $2007-08$ & 2008-09 & $2009-10$ & $2010-11$ & $2006-07$ & $2007-08$ & $2008-09$ & $2009-10$ & $2010-11$ & 2006-07 & $2007-08$ & $2008-09$ & $2009-10$ & $2010-11$ \\
\hline Kinshasa & 36,6 & 39,1 & 45,5 & 35,8 & 35,1 & 8,1 & 8,2 & 7,6 & 7,9 & 8,1 & 10,0 & 13,8 & 13,9 & 9,7 & 10,0 \\
\hline Bas-Congo & 5,0 & 5,3 & 5,2 & 4,3 & 4,4 & 5,7 & 5,4 & 5,0 & 4,9 & 4,8 & 6,4 & 6,7 & 7,0 & 5,5 & 5,2 \\
\hline Bandundu & 5,6 & 9,9 & 6,6 & 10,4 & 10,0 & 15,5 & 16,2 & 15,5 & 16,0 & 12,8 & 20,6 & 22,6 & 24,3 & 18,9 & 18,8 \\
\hline Equateur & 8,5 & 9,7 & 7,3 & 8,2 & 8,8 & 10,9 & 12,0 & 12,0 & 11,9 & 12,6 & 11,1 & 8,7 & 10,2 & 12,2 & 12,2 \\
\hline Province Orientale & 5,5 & 6,1 & 4,6 & 6,2 & 5,8 & 11,7 & 11,4 & 12,0 & 11,7 & 11,4 & 8,1 & 6,8 & 7,2 & 9,4 & 9,9 \\
\hline Nord-Kivu & 3,5 & 3,3 & 4,0 & 3,6 & 2,8 & 7,7 & 7,7 & 7,5 & 7,3 & 5,9 & 6,6 & 6,3 & 6,9 & 6,6 & 6,4 \\
\hline Sud-Kivu & 4,4 & 3,9 & 3,5 & 3,2 & 4,1 & 7,2 & 7,1 & 6,8 & 7,0 & 7,0 & 5,6 & 5,2 & 5,4 & 6,0 & 6,5 \\
\hline Maniema & 1,2 & 1,2 & 1,4 & 1,2 & 0,9 & 2,9 & 3,1 & 3,3 & 3,3 & 2,9 & 2,4 & 2,0 & 2,2 & 3,2 & 4,6 \\
\hline Kasaï-Oriental & 7,5 & 7,3 & 8,2 & 10,6 & 10,1 & 10,2 & 9,6 & 9,6 & 9,2 & 12,8 & 9,0 & 9,2 & 7,5 & 8,0 & 7,5 \\
\hline Kasaï-Occidental & 10,5 & 3,4 & 3,1 & 4,9 & 4,3 & 8,8 & 8,0 & 9,0 & 9,2 & 9,9 & 9,5 & 8,2 & 6,2 & 9,3 & 9,5 \\
\hline Katanga & 11,7 & 10,7 & 10,5 & 11,7 & 13,7 & 11,2 & 11,3 & 11,7 & 11,8 & 11,7 & 10,8 & 10,5 & 9,2 & 11,3 & 9,4 \\
\hline Total & 100,0 & 100,0 & 100,0 & 100,0 & 100,0 & 100,0 & 100,0 & 100,0 & 100,0 & 100,0 & 100,0 & 100,0 & 100,0 & 100,0 & 100,0 \\
\hline Effectifs & 2428 & 2600 & 3311 & 3048 & 3396 & 29420 & 31938 & 34512 & 35890 & 37749 & 14163 & 15231 & 16927 & 17381 & 19708 \\
\hline
\end{tabular}

Tableau 36 : Ratio classe/écoles et ratio élèves/salles de classes du pré-primaire et du primaire par province de 2006 à 2011

\begin{tabular}{|c|c|c|c|c|c|c|c|c|c|c|c|c|c|c|c|c|c|c|c|c|}
\hline \multirow{3}{*}{ Provinces } & \multicolumn{10}{|c|}{ Pré-primaire } & \multicolumn{10}{|c|}{ Primaire } \\
\hline & \multicolumn{5}{|c|}{ Ratio classe/écoles } & \multicolumn{5}{|c|}{ Ratio élèves/salles de classes } & \multicolumn{5}{|c|}{ Ratio classe/écoles } & \multicolumn{5}{|c|}{ Ratio élèves/salles de classes } \\
\hline & $\begin{array}{c}2006- \\
07\end{array}$ & $\begin{array}{c}2007- \\
08\end{array}$ & $\begin{array}{c}2008- \\
09\end{array}$ & $\begin{array}{c}2009- \\
10\end{array}$ & $\begin{array}{c}2010- \\
11\end{array}$ & $\begin{array}{c}2006- \\
07\end{array}$ & $\begin{array}{c}2007- \\
08\end{array}$ & $\begin{array}{c}2008- \\
09\end{array}$ & $\begin{array}{c}2009- \\
10\end{array}$ & $\begin{array}{c}2010- \\
11\end{array}$ & $\begin{array}{c}2006- \\
07\end{array}$ & $\begin{array}{c}2007- \\
08\end{array}$ & $\begin{array}{c}2008- \\
09\end{array}$ & $\begin{array}{c}2009- \\
10\end{array}$ & $\begin{array}{c}2010- \\
11\end{array}$ & $\begin{array}{c}2006- \\
07\end{array}$ & $\begin{array}{c}2007- \\
08\end{array}$ & $\begin{array}{c}2008- \\
09\end{array}$ & $\begin{array}{c}2009- \\
10\end{array}$ & $\begin{array}{c}2010- \\
11\end{array}$ \\
\hline Kinshasa & 3 & 3 & 3 & 3 & 3 & 23 & 24 & 24 & 23 & 22 & 9 & 9 & 9 & 9 & 8 & 37 & 40 & 39 & 38 & 37 \\
\hline Bas-Congo & 3 & 3 & 3 & 3 & 3 & 19 & 21 & 22 & 22 & 21 & 9 & 9 & 8 & 8 & 9 & 33 & 37 & 37 & 37 & 39 \\
\hline Bandundu & 3 & 3 & 3 & 3 & 3 & 24 & 27 & 27 & 27 & 26 & 8 & 8 & 8 & 7 & 9 & 28 & 31 & 31 & 30 & 31 \\
\hline Equateur & 3 & 3 & 3 & 3 & 3 & 29 & 29 & 27 & 26 & 26 & 8 & 7 & 7 & 7 & 7 & 36 & 37 & 35 & 36 & 38 \\
\hline Province Orientale & 3 & 3 & 3 & 3 & 3 & 25 & 25 & 24 & 25 & 24 & 8 & 8 & 8 & 8 & 8 & 40 & 41 & 39 & 39 & 41 \\
\hline Nord-Kivu & 3 & 3 & 3 & 3 & 3 & 27 & 27 & 31 & 28 & 28 & 8 & 9 & 8 & 9 & 9 & 43 & 44 & 43 & 44 & 44 \\
\hline Sud-Kivu & 2 & 2 & 3 & 2 & 2 & 30 & 32 & 32 & 31 & 30 & 8 & 8 & 8 & 8 & 8 & 44 & 46 & 43 & 42 & 41 \\
\hline Maniema & 3 & 3 & 3 & 3 & 3 & 25 & 26 & 25 & 23 & 26 & 8 & 7 & 7 & 7 & 7 & 37 & 38 & 35 & 34 & 36 \\
\hline Kasaï-Oriental & 2 & 3 & 2 & 3 & 3 & 28 & 28 & 27 & 27 & 28 & 7 & 7 & 7 & 7 & 6 & 43 & 43 & 40 & 41 & 40 \\
\hline Kasaï-Occidental & 3 & 3 & 3 & 3 & 3 & 25 & 30 & 25 & 23 & 22 & 7 & 7 & 7 & 7 & 7 & 40 & 39 & 38 & 37 & 38 \\
\hline Katanga & 3 & 3 & 3 & 3 & 3 & 34 & 34 & 36 & 29 & 29 & 8 & 8 & 8 & 8 & 8 & 41 & 46 & 44 & 43 & 44 \\
\hline Total & 3 & 3 & 3 & 3 & 3 & 26 & 27 & 26 & 25 & 25 & 8 & 8 & 8 & 8 & 8 & 38 & 40 & 38 & 38 & 39 \\
\hline
\end{tabular}

Source : Données des annuaires statistiques DEP/EPSP, RDC 2006-07, 2007-08, 2008-09, 2009-10 et 2010-11 
L'appréciation de la qualité des salles de classe révèle que globalement leur état s'est plutôt dégradé au fil du temps, passant de $85 \%$ de salles de classe en bon état en 2006-2007 à 79,0 \% en 2010-2011 (graphique 19). La dégradation des salles de classe est très marquée dans le Sud-Kivu, résultat sans doute en lien avec les conflits et l'instabilité qu'a connus cette partie du territoire. D'une manière générale, au niveau de l'ensemble du pays et dans la plupart des provinces, on note une dégradation de la qualité des infrastructures.

Graphique 19 : Evolution de la proportion (en \%) des salles de classe du primaire en bon état par province

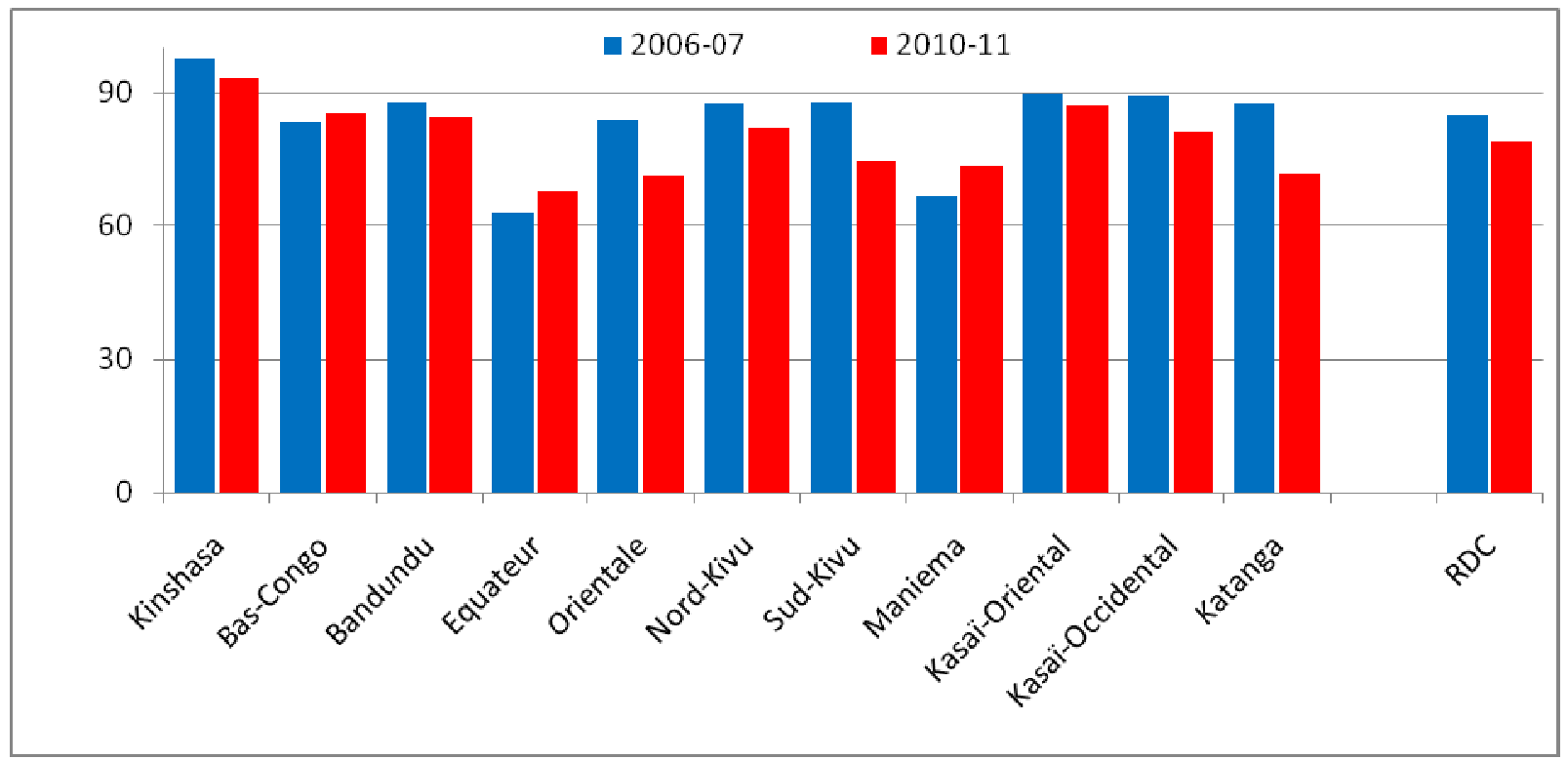

Source : Données des annuaires statistiques DEP/EPSP, RDC 2006-07 et 2010-11

\section{Le secondaire}

Le nombre d'établissements secondaires est passé de 14163 à 19708 au cours des cinq années (20062011), soit un accroissement absolu de 5545 établissements (39,2\%). es établissements secondaires sont encore plus inégalement répartis entre les provinces (tableau 35). La province du Bandundu concentre à elle seule près d'un établissement sur cinq $(18,8 \%)$, suivie de Kinshasa $(10,0 \%)$ et de l'Equateur (12,2\%).

Comme pour les écoles primaires, la dégradation des infrastructures au cours des cinq ans est aussi notable avec le même schéma observé précédemment (graphique 20). La dégradation est encore très nette dans la province du Sud-Kivu.

Graphique 20 : Evolution de la proportion (en \%) des salles de classe du secondaire en bon état par province

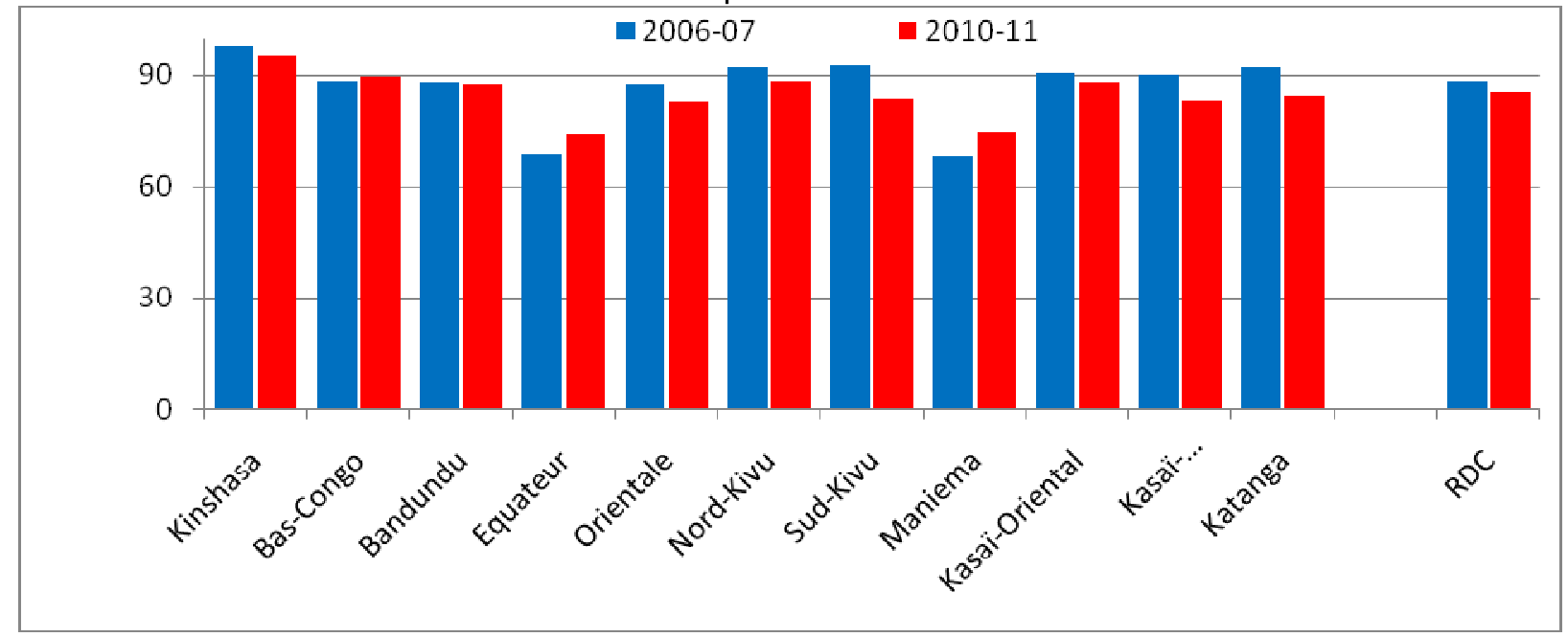

Source : Données des annuaires statistiques DEP/EPSP, RDC 2006-07 et 2010-11 
On note toutefois une amélioration dans trois provinces (Maniema, Equateur et Bas Congo) aussi bien au primaire qu'au secondaire.

\section{b) Appréciation de l'offre en personnel}

\section{Le pré-primaire}

Le nombre d'éducateurs est passé de 6744 en 2006-2007 à 9775 en 2010-2011, soit une augmentation de $44,9 \%$ au cours des cinq années ( 3031 en termes absolus). Cette forte augmentation du nombre d'éducateurs du pré-primaire a été au bénéfice de la ville province de Kinshasa (tableau 37). Les provinces avec de grands centres urbains sont les mieux dotées en personnel.

Le primaire

La répartition des enseignants du primaire est presqu'analogue à celle des écoles. C'est dans les provinces où il y a le plus d'écoles que l'on trouve le plus d'enseignants. L'évolution du nombre d'enseignants en termes relatifs, est restée presque stable pour les provinces. Le Bandundu est encore la province où il y a le plus d'enseignants. A l'opposé, le Kasaï oriental, qui a connu une baisse très importante de ses effectifs d'enseignants, est la province où il y a moins d'enseignants.

\section{Le secondaire}

La répartition des enseignants du secondaire par province n'est guère différente de celle des enseignants du primaire. On note cependant que les provinces du Bandundu et de l'Equateur disposent du plus grand nombre d'enseignants du secondaire. 
Tableau 37 : Répartition (en \%) des éducateurs, des enseignants du primaire et des enseignants du secondaire par province de 2006 à 2011

\begin{tabular}{|c|c|c|c|c|c|c|c|c|c|c|c|c|c|c|c|}
\hline \multirow[b]{2}{*}{ Provinces } & \multicolumn{5}{|c|}{ Educateurs } & \multicolumn{5}{|c|}{ Enseignants du primaire } & \multicolumn{5}{|c|}{ Enseignants du secondaire } \\
\hline & $\begin{array}{c}2006- \\
07\end{array}$ & $\begin{array}{c}2007- \\
08\end{array}$ & $\begin{array}{c}2008- \\
09\end{array}$ & $\begin{array}{c}2009- \\
10\end{array}$ & $\begin{array}{c}2010- \\
11\end{array}$ & $\begin{array}{c}2006- \\
07\end{array}$ & $\begin{array}{c}2007- \\
08\end{array}$ & $\begin{array}{c}2008- \\
09\end{array}$ & $\begin{array}{c}2009- \\
10\end{array}$ & $\begin{array}{c}2010- \\
11\end{array}$ & $\begin{array}{c}2006- \\
07\end{array}$ & $\begin{array}{c}2007- \\
08\end{array}$ & $\begin{array}{c}2008- \\
09\end{array}$ & $\begin{array}{c}2009- \\
10\end{array}$ & $\begin{array}{c}2010- \\
11\end{array}$ \\
\hline Kinshasa & 33,7 & 36,8 & 42,1 & 33,7 & 32,6 & 9,0 & 9,0 & 8,8 & 8,9 & 9,5 & 11,7 & 10,9 & 9,0 & 12,6 & 12,4 \\
\hline Bas-Congo & 4,6 & 4,9 & 4,5 & 4,1 & 4,2 & 6,1 & 5,9 & 5,4 & 5,3 & 5,4 & 6,2 & 6,2 & 5,6 & 6,3 & 5,8 \\
\hline Bandundu & 6,1 & 11,0 & 9,5 & 13,0 & 11,6 & 15,1 & 15,8 & 15,0 & 15,5 & 14,6 & 21,8 & 21,2 & 19,4 & 21,0 & 21,5 \\
\hline Equateur & 10,1 & 10,4 & 8,4 & 9,3 & 9,6 & 10,6 & 11,4 & 11,4 & 11,3 & 12,2 & 10,7 & 12,9 & 12,6 & 9,9 & 10,3 \\
\hline Province Orientale & 5,9 & 6,3 & 4,6 & 6,0 & 5,7 & 11,7 & 11,3 & 11,9 & 11,8 & 11,9 & 8,5 & 8,7 & 9,4 & 8,5 & 9,0 \\
\hline Nord-Kivu & 5,1 & 4,7 & 4,6 & 3,7 & 3,5 & 8,3 & 8,5 & 8,3 & 8,4 & 7,0 & 6,5 & 6,7 & 6,5 & 7,2 & 6,9 \\
\hline Sud-Kivu & 3,6 & 3,6 & 3,1 & 3,2 & 3,4 & 7,1 & 7,1 & 6,8 & 7,0 & 7,0 & 5,8 & 5,7 & 6,1 & 5,7 & 6,1 \\
\hline Maniema & 1,2 & 1,1 & 1,4 & 1,4 & 0,9 & 2,6 & 2,7 & 2,9 & 3,0 & 2,7 & 2,9 & 2,8 & 3,3 & 2,9 & 4,2 \\
\hline Kasaï-Oriental & 6,4 & 6,6 & 7,0 & 9,2 & 9,0 & 9,6 & 8,9 & 0,9 & 8,3 & 8,5 & 7,9 & 7,3 & 8,2 & 7,5 & 7,1 \\
\hline Kasaï-Occidental & 10,4 & 3,3 & 2,4 & 4,2 & 3,8 & 8,2 & 7,4 & 8,3 & 8,4 & 9,1 & 7,5 & 7,5 & 8,7 & 8,0 & 8,0 \\
\hline Katanga & 13,0 & 11,3 & 12,3 & 12,1 & 15,8 & 11,7 & 12,0 & 12,2 & 12,1 & 12,1 & 10,5 & 10,0 & 11,3 & 10,6 & 8,7 \\
\hline Total & 100,0 & 100,0 & 100,0 & 100,0 & 100,0 & 100,0 & 100,0 & 100,0 & 100,0 & 100,0 & 100,0 & 100,0 & 100,0 & 100,0 & 100,0 \\
\hline Effectifs & 6744 & 7241 & 10139 & 8585 & 9775 & 230834 & 255594 & 274453 & 285620 & 296554 & 179635 & 188808 & 212273 & 218320 & 248591 \\
\hline
\end{tabular}

Source : Données des annuaires statistiques DEP/EPSP, RDC 2006-07, 2007-08, 2008-09, 2009-10 et 2010-11 


\section{c) Inadéquation des infrastructures et des contenus à certains groupes d'enfants vulnérables}

La faible participation scolaire des enfants vivant avec un handicap est le reflet d'une inadaptation de l'offre scolaire (infrastructures et contenu pédagogique) aux besoins de ce groupe d'enfants. Les résultats de l'EADE 2012 révèlent que 1,3\% des enfants de 5-17 ans, soit un effectif absolu de 299 998 enfants, vivent avec un handicap. Parmi les enfants vivants avec un handicap, les handicapés des membres inférieurs représentent la grande part (34,2\%). Les enfants atteints de déficience mentale représentent $30,9 \%$. II existe quelques légères variations selon le sexe et le milieu de résidence quant à la nature du handicap (tableau 38).

Tableau 38 : Répartition (en \%) des enfants de 6-17 ans ayant un handicap selon le handicap

\begin{tabular}{lccccc}
\hline Principal handicap & Masculin & Féminin & Urbain & Rural & Ens RDC \\
\hline Sourd & 12,3 & 15,6 & 13,2 & 14,0 & 13,7 \\
Muet & 11,4 & 9,9 & 7,1 & 12,9 & 10,8 \\
Mal-voyant & 7,1 & 4,9 & 9,5 & 4,2 & 6,2 \\
Aveugle & 1,4 & 3,2 & - & 3,4 & 2,2 \\
Handicap mbre sup. & 13,3 & 19,3 & 18,4 & 14,4 & 15,8 \\
Handicap mbre inf. & 22,7 & 15,7 & 17,3 & 21,2 & 19,7 \\
Déficient mental & 18,4 & 17,0 & 13,3 & 20,4 & 17,8 \\
Autre & 13,3 & 14,5 & 21,2 & 9,5 & 13,8 \\
\hline Total & 100,0 & 100,0 & 100,0 & 100,0 & 100,0 \\
Effectif & 173176 & 126820 & 110650 & 189348 & 299998 \\
\hline \% parmi 5-17 ans & 1,5 & 1,2 & 1,4 & 1,3 & 1,3 \\
\hline \% parmi handicapés & 28,0 & 21,8 & 30,3 & 22,7 & 25,0 \\
\hline \multicolumn{5}{l}{ Source : Données de l'enquête auprès des ménages, EADE-RDC 2012 } \\
\multicolumn{7}{c}{ - Effectifs faibles }
\end{tabular}

En examinant le phénomène de l'exclusion scolaire selon le handicap (graphique 21), on note, comme on pouvait s'y attendre, que les enfants n'ayant aucun handicap, sont ceux qui sont le moins exclus du système éducatif (28,7 \% d'EADE au niveau de l'ensemble du pays). A l'autre extrémité, les enfants atteints de cécité sont plus en dehors de l'école. Après les enfants aveugles viennent les enfants muets avec la plus grande proportion d'EADE (82,5\%), suivis des enfants atteints de déficience mentale $(74,9 \%$ d'EADE), ceux atteints de surdité $(60,1 \%)$, ayant un handicap des membres inférieurs (46,8\%), ayant un handicap des membres supérieurs $(32,5 \%)$ et les enfants malvoyants $(31,1 \%)$. Cette hiérarchisation du niveau d'exclusion des enfants en fonction de la nature du handicap présente des différences entre filles et garçons d'une part (graphique 21) et entre milieu urbain et milieu rural d'autre part (graphique 22). Ainsi, la proportion des filles en dehors de l'école dans la catégorie " autre handicap " ${ }^{24}$ est nettement plus élevée $(57,6 \%)$ que ce qu'elle est chez les garçons $(29,2 \%)$. En outre, la proportion de filles mal-voyantes en dehors de l'école est de $45,7 \%$ contre $24,2 \%$ chez les garçons mal-voyants. Par ailleurs, la proportion d'enfants mal-voyants en dehors de l'école en milieu rural $(46,5 \%)$ est largement supérieure à celle observée chez les malvoyants en milieu urbain $(18,2 \%)$.

\footnotetext{
${ }^{24}$ Cette catégorie « autre handicap » qui représente $14,4 \%$ des handicaps n'a pas été précisée lors de la collecte.
} 
Graphique 21 : Proportion (en \%) des enfants scolarisables (5-17 ans) en dehors de l'école selon la nature du handicap ${ }^{25}$ et par sexe

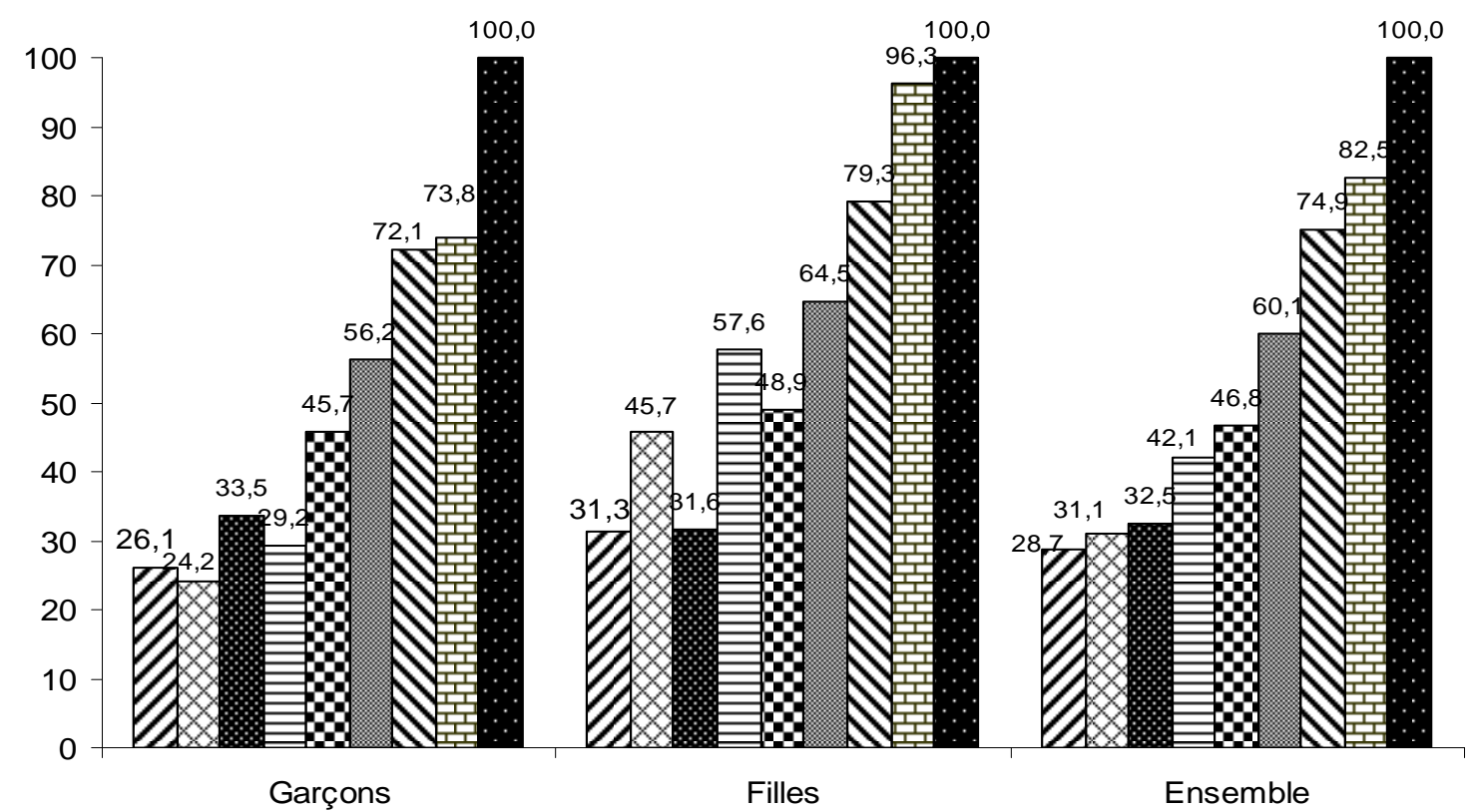

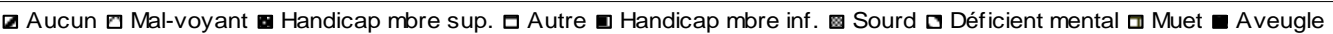

Source : Données de l'enquête auprès des ménages, EADE-RDC 2012

Graphique 22 : Proportion (en \%) des enfants scolarisables (5-17 ans) en dehors de l'école selon la nature du handicap et par milieu de résidence

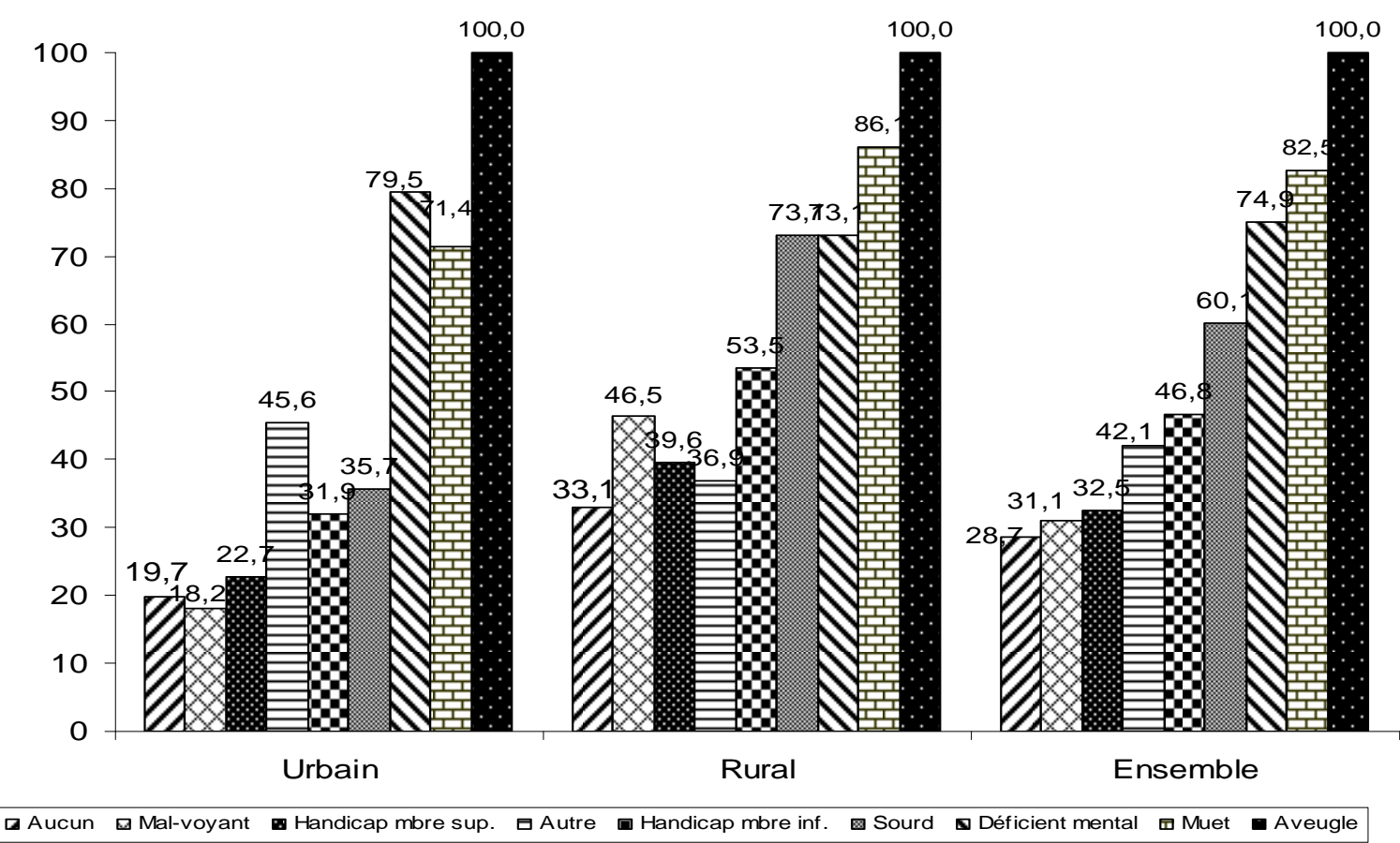

Source : Données de l'enquête auprès des ménages, EADE-RDC 2012

\footnotetext{
${ }^{25}$ Du fait des faibles effectifs d'enfants aveugles dans l'échantillon, ils apparaissent tous comme des EADE. D'où cette proportion de $100 \%$.
} 


\section{e) Dotation en manuels scolaires}

Le diagnostic du sous-programme fourniture de supports pédagogiques du Plan intérimaire de l'éducation 2012/2014 reconnait que malgré l'absence de politique nationale dans le domaine de manuels scolaires, des efforts sont entrepris au plan national et ce, depuis 2004 pour doter les établissements. L'objectif poursuivi étant d'améliorer, entre autres, les conditions d'apprentissage par la fourniture de matériels et de supports pédagogiques.

L'enquête sur la situation des enfants et adolescents en dehors de l'école a permis de recueillir des informations sur la dotation des élèves en manuels scolaires ${ }^{26}$. Le tableau 39 indique qu'au plan national, environ 1 enfant sur $5(22,3 \%)$ a déclaré que l'école donnait des manuels scolaires dont $21,7 \%$ auraient reçu une dotation partielle et $0,6 \%$ une dotation complète (tous les manuels).

Tableau 39 : Répartition (en \%) des enfants et des adolescents de 7-12 ans scolarisés au primaire en 2010-2011 selon la dotation en manuels scolaires par milieu de résidence et par sexe

\begin{tabular}{lccccccccc}
\hline Dotation en & \multicolumn{3}{c}{ Garçons } & \multicolumn{3}{c}{ Filles } & \multicolumn{3}{c}{ Ensemble } \\
\cline { 2 - 10 } manuels & Urbain & Rural & Ens. & Urbain & Rural & Ens. & Urbain & Rural & Ens. \\
\hline Aucun & 79,5 & 77,0 & 77,9 & 80,8 & 75,5 & 77,5 & 80,1 & 76,3 & 77,7 \\
Une partie & 19,7 & 22,6 & 21,5 & 18,4 & 24,0 & 21,8 & 19,0 & 23,2 & 21,7 \\
Tous & 0,9 & 0,4 & 0,6 & 0,8 & 0,6 & 0,7 & 0,8 & 0,5 & 0,6 \\
\hline Total & 100,0 & 100,0 & 100,0 & 100,0 & 100,0 & 100,0 & 100,0 & 100,0 & 100,0 \\
Effectifs & 1622996 & 2912283 & 4535279 & 1612289 & 2557830 & 4170119 & 3235285 & 5470113 & 8705399 \\
\hline
\end{tabular}

Source : Données de l'enquête auprès des ménages, EADE-RDC 2012

Selon le milieu de résidence, il ressort que 0,8\% aurait reçu tous les manuels en ville contre seulement $0,5 \%$ en milieu rural. À l'opposé, la dotation partielle est une pratique plus répandue en milieu rural $(23,2 \%)$ comparativement au milieu urbain $(19,0 \%)$.

La province Orientale et celle de Kinshasa présentent les pourcentages les plus élevés d'enfants ayant déclaré avoir reçu tous les manuels avec respectivement $2,7 \%$ et $1,6 \%$ (tableau 40). La dotation partielle est plus courante au Kasaï Occidental (43,8 \%), au Bandundu (34,2 \%), au Sud-Kivu $(32,7 \%)$ et dans une moindre mesure au Nord-Kivu, dans la Province Orientale, à l'Equateur et à Kinshasa avec des proportions variant entre 17,0\% (Kinshasa) et 26,1\% (Nord-Kivu). C'est au Maniema (93,4 \%), au Kasaï Oriental (90,7 \%), au Bas-Congo (89,7\%) et au Katanga (90,7 \%) qu'on observe les fortes proportions d'enfants ayant déclaré n'avoir pas reçu de manuels scolaires. La comparaison des pourcentages par province d'enfants ayant reçu tous les manuels scolaires donne un maximum de 1,6\% (Kinshasa).

\footnotetext{
${ }^{26}$ II convient de souligner que la question a été posée dans le sens de donner les manuels aux enfants, ce qui permet leur utilisation à domicile. Mais il semble que l'objectif de la politique était de rendre les manuels disponibles à l'école pour les élèves et non pas les leur donner. Cet aspect n'a pas été bien clarifié par les acteurs du système lors de l'atelier de validation des outils de collecte. On aurait pu alors poser une autre question sur la « disponibilité des manuels à l'école ».
} 
Tableau 40 : Répartition (en \%) des enfants et des adolescents de 7-12 ans scolarisés au primaire en 2010-2011 selon la dotation en manuels scolaires par province

\begin{tabular}{lcccccrrr}
\hline \multirow{2}{*}{ Provinces } & \multicolumn{9}{c}{ Pourcentages } \\
\cline { 2 - 9 } & Aucun & Une partie & \multicolumn{1}{c}{ Tous } & Total & \multicolumn{1}{c}{ Aucun } & Une partie & \multicolumn{1}{c}{ Tous } & \multicolumn{1}{c}{ Total } \\
\hline Kinshasa & 81,4 & 17,0 & 1,6 & 100,0 & 1064259 & 222720 & 20314 & 1307293 \\
Bas-Congo & 89,7 & 10,0 & 0,3 & 100,0 & 449088 & 50069 & 1498 & 500655 \\
Bandundu & 65,7 & 34,2 & 0,1 & 100,0 & 683131 & 356173 & 1093 & 1040397 \\
Equateur & 75,7 & 24,2 & 0,0 & 100,0 & 709561 & 226955 & 336 & 936852 \\
Orientale & 71,0 & 26,3 & 2,7 & 100,0 & 715442 & 264726 & 27098 & 1007266 \\
Nord Kivu & 73,7 & 26,1 & 0,2 & 100,0 & 455212 & 161238 & 1083 & 617533 \\
Maniema & 93,4 & 6,4 & 0,2 & 100,0 & 239976 & 16577 & 476 & 257029 \\
sud Kivu & 67,2 & 32,7 & 0,0 & 100,0 & 405913 & 197465 & 218 & 603596 \\
Katanga & 90,2 & 9,8 & - & 100,0 & 1107067 & 120068 & - & 1227135 \\
Kasaï oriental & 90,7 & 9,3 & - & 100,0 & 678473 & 69397 & - & 747870 \\
Kasaï occidental & 56,0 & 43,8 & 0,2 & 100,0 & 257538 & 201503 & 732 & 459773 \\
\hline Total & 77,7 & 21,7 & 0,6 & 100,0 & 6765660 & 1886891 & 52848 & 8705399 \\
\hline
\end{tabular}

Source : Données de l'enquête auprès des ménages, EADE-RDC 2012

\section{f) Distance à l'école}

La question a été posée au ménage de savoir quelle est la distance (en kms) pour se rendre à l'école la plus proche (en distinguant pour chaque type d'école : pré-scolaire, primaire et secondaire). Un regroupement des distances en classes significatives a été ensuite faite. Comme le montre le graphique 23 , excepté en milieu urbain chez les 12-17 ans ${ }^{27}$, d'une manière générale (et surtout en milieu rural), plus la distance à l'école est élevée, plus la proportion des EADE est élevée.

Graphique 23 : Proportion (en \%) des EADE selon la distance à l'établissement le plus proche

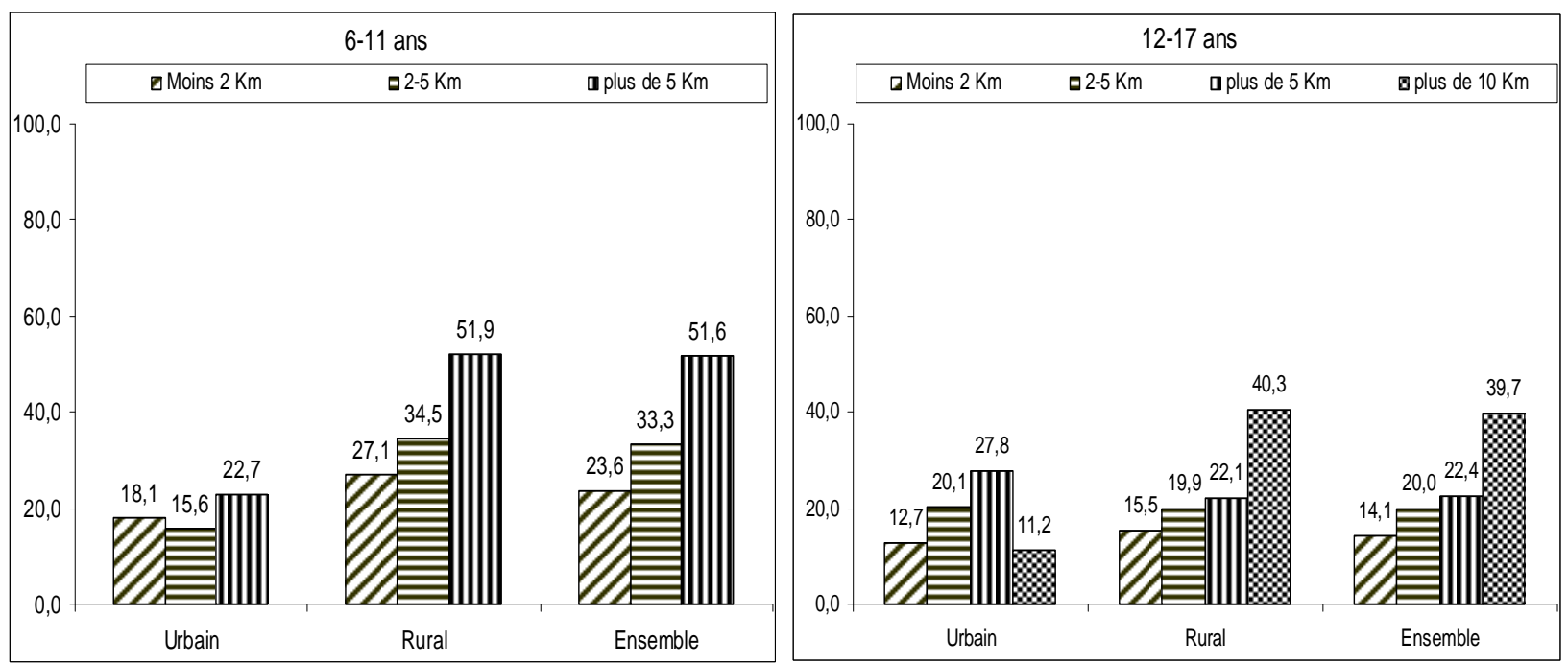

Source : Données de l'enquête auprès des ménages, EADE-RDC 2012

Les résultats des analyses multivariées confirment cette importance de la question de la distance (et donc de la disponibilité des infrastructures), comme un des déterminants de la participation scolaire des enfants et des adolescents en milieu rural. En effet, la distance à l'école primaire occupe le premier rang comme déterminant en milieu rural. Elle est déterminante dans 8 provinces sur les 11 : Bandundu, Province orientale, Maniema et Kasaï occidental $\left(1^{\text {er }}\right.$ rang), Nord Kivu et Katanga ( $2^{\text {ème }}$ rang), Equateur et Sud Kivu (3 ${ }^{\text {ème }}$ rang). En ce qui concerne le secondaire, la distance à

\footnotetext{
${ }^{27}$ Le fait que la proportion des EADE soit si faible en milieu urbain pour les $12-17$ ans pour la classe « $10 \mathrm{~km}$ et plus » peut être dû à un problème de faible effectif.
} 
l'établissement occupe le $3^{\text {ème }}$ rang en milieu rural. Elle occupe le premier rang dans le Bandundu, l'Equateur et la Province orientale et le $3^{\text {ème }}$ rang dans le Katanga (tableau A29 en annexe). L'exemple de l'Equateur est assez parlant, en ce sens que, bien que ce soit une des provinces où la proportion de ménages pauvres est la plus élevée, on note que ce n'est pas le revenu qui est le premier facteur déterminant au niveau du secondaire, mais plus tôt la disponibilité de l'offre, notamment la distance à l'école.

En effet, la raison la plus citée après la barrière financière pour justifier la non scolarisation des enfants est la distance ou l'absence de l'école, évoquée dans $21,0 \%$ des cas (tableau A30 en annexe). Les discours recueillis lors des entretiens qualitatifs vont dans ce sens :

"Les raisons sont multiples, il y a la pauvreté, il y a la mauvaise foi de certains parents. Il y a aussi la distraction des enfants eux-mêmes. Et parfois le manque des infrastructures aussi: vous vous retrouvez dans un milieu où il n'y a pas d'écoles. Pour que les enfants accèdent à l'école, ils doivent parcourir une distance de 15 à $20 \mathrm{~km}$. Et la conséquence, les petits enfants de 5 à 6 enfants n'étudient pas. Pour étudier, il faut avoir un ménage d'un ami, d'un frère pour les confier, un ménage qui est dans ce milieu là. Ce sont les raisons qui font que les enfants ne puissent pas étudier " (homme, parlementaire).

\subsubsection{Déterminants au niveau politique, gouvernance et financement}

\section{a) Faible financement du secteur de l'éducation/Effectivité partielle de la gratuité scolaire}

Le poids énorme des dépenses scolaires pour les ménages congolais est en en grande partie dû à la faiblesse des financements publics dans le secteur de l'éducation. En mettant en lien les dépenses totales d'éducation des ménages en 2010-2011 avec le budget de l'EPSP (tableau 41), il ressort que les ménages dépensent près de trois (3) fois plus que l'Etat dans l'éducation (rapport de 2,7). La part du budget de l'Etat consacré au secteur de l'EPSP demeure faible $(6,7 \%)$ et lorsque qu'on met en rapport la contribution financière des ménages à l'éducation des enfants de 3-17 ans, on note que cette contribution représente $17,9 \%$ du budget 2011 de l'Etat. Une fois encore ces résultats montrent combien la contribution des ménages congolais à l'éducation des enfants est énorme au regard du financement de l'Etat.

Tableau 41 : Rapport entre les dépenses d'éducation des ménages et le budget de l'éducation (Montants en USD)

\begin{tabular}{lr}
\hline \multicolumn{1}{c}{ Rubriques } & \multicolumn{1}{c}{ Montant } \\
\hline Budget EPSP 2011 & 504362222 \\
Budget national 2011 & 7495555556 \\
Dépenses d'éducation des ménages en 2010-2011 (EADE) & 1344403019 \\
Part du budget de l'EPSP dans le budget national & $6,7 \%$ \\
Ce que représentent les dépenses d'éducation des ménages par & $17,9 \%$ \\
rapport au budget national & 2,7 \\
Rapport entre dépenses d'éducation des ménages et budget EPSP & \\
\hline
\end{tabular}

Note : Source données sur le budget : Plan intérimaire de l'éducation 2012/2014. Conversion des montants en dollars US par les auteurs ( 1 USD=900 Francs Congolais)

Par ailleurs, la gratuité de l'éducation primaire (notamment pour les quatre premières années du primaire) est loin d'être totalement effective. Par ailleurs, les acteurs rencontrés souhaitent la généralisation de cette mesure à tout le cycle primaire et sur l'ensemble du pays.

"La politique de la gratuité, c'était une bonne politique puisque ça permet d'atteindre les objectifs du millénaire puisqu'on a dit que tous les enfants soient à l'école d'ici 2015. Mais les mesures d'encadrement n'ont pas suivi. Imaginez avec les parents ici un enseignant peut facilement avoir ici au Nord Kivu 150\$, avec le salaire de l'État, vous demandez à cet enseignant là de redescendre à 50\$... Donc les gens vont partir chercher mieux ailleurs. Et ce sont les enfants qui vont souffrir. II faut que l'État prenne ses responsabilités en main pour donner un salaire qui convient à l'enseignant et là les objectifs seront atteints. Puisqu'à l'intérieur, là où les parents sont démunis si les écoles sont payées, 
on n'a pas besoin de prime des parents, là on accepte tous les enfants. " (Homme, cadre du système éducatif, Nord Kivu).

\section{b) Faible capacité institutionnelle et humaine}

Ce problème de capacité institutionnelle et humaine se pose à plusieurs niveaux : au niveau des comités de gestion mis en place dans les écoles, au niveau de la gestion des ressources humaines et au niveau de la coordination et du pilotage du système éducatif.

\section{Au niveau des comités de gestion}

Dans presque toutes les provinces visitées, les acteurs soulignent des disfonctionnement dans la gestion des frais de fonctionnement des écoles. Sont notamment soulignés la faible capacité de gestion des comités de gestion mis en place dans les écoles, le manque de concertation et de transparence dans la gestion des fonds.

"Là il faut renforcer la capacité des gestionnaires des écoles. Très peu d'écoles ont cette culture de fonctionner avec la provision budgétaire, même pas élaboré...Pourtant, vous voyez même le Préfet élabore le budget lui-même, il est lui-même élaborateur, l'ordonnateur, ça ne peut pas se faire comme ça. Le budget doit être la carte visualisée de l'école, il faut impliquer le comité des parents... Le préfet doit respecter le besoin budgétaire, l'école c'est une entreprise qui doit avoir une comptabilité et aussi un suivi et un contrôle » (Homme, cadre du système éducatif).

Les propos suivants d'un enseignant montrent que toutes les composantes du comité de gestion ne participent pas à la gestion des écoles comme prévu : "Bon! Le fonctionnement des frais des écoles, moi en tant qu'enseignant, ce sont des chefs d'établissement qui gèrent. Nous, nous sommes de simples observateurs. Les chefs d'établissement qui gèrent cet argent qui sont contrôlés et qui ont des détails, qu'est ce qu'ils font avec cet argent? Mais, je connais bien qu'il y a certains chefs d'établissement qui gèrent très bien cet argent, il y a ceux qui ne gèrent même pas cet argent et ils pensent que c'est leur argent. Or, c'est pour faire fonctionner l'établissement public de l'État pour que l'établissement puisse avancer. C'est un problème d'homme. II y a ceux qui gèrent très bien, ils sont minoritaires, mais les autres cherchent à s'en accaparer, c'est leur propre argent " (Homme, enseignant, Kinshasa).

\section{Au niveau de la gestion des ressources humaines}

En ce qui concerne la gestion des ressources humaines du secteur de l'éducation, en plus du problème des salaires des enseignants déjà mentionné, on souligne plusieurs autres difficultés. II s'agit notamment du manque de formation de certains enseignants, du non renouvellement du personnel enseignant, de l'absence d'un système de retraite pour les enseignants, etc. On souligne aussi la dévalorisation de la fonction d'enseignant et le manque de motivation des enseignants actuels avec pour conséquences les fréquentes absences des enseignants dans les écoles et l'abandon de la profession par plusieurs enseignants. Ces difficultés, mentionnées dans les propos ci-dessous, ont des effets négatifs sur la qualité de l'enseignement et la réussite scolaire.

"Bon! La gestion de ressources humaines pose problème, la gestion de ressources humaines, parce qu'aujourd'hui nous avons des gens fatigués, qui attendent la pension et ceux qui meurent, 6 mois après, il n'y a rien! Pas de service de rente survie, donc, la gestion de ressources humaines pose problème. II y a des agents, depuis deux ans, ils sont impayés, alors qu'est ce qu'on va demander aux gens qui viennent une fois par semaine... II y en a qui viennent de très loin pour travailler ici, ils doivent payer le transport ? Et avec quel salaire, donc, tout ça a un impact négatif sur le rendement des agents... Voyez! II faudra améliorer les conditions de travail pour que les ressources humaines puissent donner le meilleur d'elles-mêmes, qu'ils soient un peu bien rémunérés pour ne pas dire très bien rémunérés » (homme, cadre du système éducatif, Kinshasa).

"La gestion des ressources humaines, il y a bien sûr des failles. En matière de recrutement dans les écoles publiques, je souhaiterai qu'on fasse des concours, d'ailleurs c'est ce qui est prévu dans le statut des agents de carrière afin que l'on puisse prendre ceux-là qui méritent réellement. Le concours ne se fait pas, ça c'est une faiblesse, c'est vraiment une faiblesse... La gestion des ressources humaines aussi en termes de salaire, parfois on ne donne pas un salaire suffisant aux enseignants, l'État ne donne pas ce qui est suffisant. Ce que les parents donnent parfois ce n'est pas aussi bien réparti, ce n'est pas équitablement réparti... Quand un enseignant tombe malade il doit se débrouiller... II n'y a pas de sécurités sociales, en termes de vieillesse il n'y a pas de sécurité sociale, 
l'enseignant est un laissé pour compte... En matière de formation aussi, évidement il y a les activités de SERNAFOR (service de formation des enseignants) qui permettent aux enseignants de pouvoir s'auto former, on essaie de gérer ça comme il faut... Mais quelque part, c'est un peu désuet, dépassé, parce que c'est comme une formalité qui n'a pas de raison d'être, parce que souvent il n'y a pas d'outil de formation... Cela n'a pas vraiment un impact très visible » (Homme, enseignant, Nord Kivu).

\section{Au niveau de la coordination et de la gestion du système éducatif}

La réussite des politiques et stratégies éducatives nécessite une bonne coordination des actions et une réelle concertation entre les différents acteurs du système éducatif. Cependant, globalement, on souligne une insuffisance de la communication entre les différents acteurs du niveau central et ceux du niveau provincial comme l'indiquent les propos suivant d'un responsable provincial de l'éducation. "Peut-être au niveau national, il y a quand même une collaboration, tandis que chez nous, au niveau provincial comme local, il n'y a pas une collaboration entre ces différents systèmes de pilotage (homme, cadre du système éducatif, Nord Kivu). Ces propos traduisent le manque de concertation d'une part entre les acteurs du niveau central et ceux du niveau déconcentré, et entre les acteurs au niveau provincial d'autre part. Cette situation explique le faible engagement de certains acteurs quant à la mise en œuvre des politiques éducatives. Pour remédier à cette situation, et favoriser la circulation de l'information à tous les niveaux, certains acteurs proposent la mise en place d'un mécanisme de communication au niveau du ministère de l'éducation avec des représentations au niveau provincial.

Par ailleurs, le système d'information et de gestion de l'éducation (SIGE), mis en place ces dernières années pour traiter et publier les données scolaires, constitue un moyen essentiel pour la diffusion des statistiques scolaires et leurs prises en compte dans les programmes de l'éducation. Cependant, on constate que le SIGE est méconnu même des principaux acteurs du système éducatif. De plus, les acteurs relèvent plusieurs faiblesses dans le fonctionnement du SIGE. Certains acteurs du niveau central et déconcentré soulignent la faible distribution des annuaires statistiques. Ils soulignent aussi l'insuffisance des moyens logistiques (ordinateurs, moyens de déplacement, etc.) pour la collecte de l'information au niveau provincial. Cette situation explique sans doute en partie le retard constaté dans la production des annuaires. Les propos suivants illustrent la faible diffusion des annuaires scolaires.

"On nous demande de faire les statistiques, nous les envoyons à la division, souvent un annuaire pour la division, pour la province éducationnelle, mais le document qui centralise pour tout le pays, ça ne nous revient pas, ça n'arrive pas, moi, je n'ai jamais vu ça. Moi, l'annuaire que j'ai, c'est l'annuaire de la division provinciale pour la province éducationnelle mais le ministère édite un document dans ce sens là, je ne l'ai jamais vu » (Homme, cadre du système éducatif, Nord Kivu).

Du point de vue des PTF interviewés, il existe un bon système de concertation au sein des PTF qui interviennent dans le secteur de l'éducation. "Jusqu'à preuve du contraire le pilotage se fait bien. D'ailleurs, il y a des initiatives afin de pouvoir cibler tous les partenaires qui interviennent dans le secteur de l'éducation, pour qu'il y ait cette coordination efficace de toutes les interventions des partenaires dans le secteur » (Homme, personnel de PTF). Cependant, ce système est peu connu des autres acteurs surtout au niveau déconcentré, qui pensent que les PTF agissent sans concertation entre eux et sans implication réelle des acteurs locaux. "Le système de pilotage, je crois qu'il y a certaines choses qu'il faut essayer de cadrer, ce système doit aussi obéir à la politique de la décentralisation. Il y a comme une sorte d'écart, il y a des programmes qui sont conçus au niveau national, mais qui doivent aussi s'appliquer au niveau des provinces. Sinon il y aura une cassure. A Kinshasa, avec la proximité il n'y a pas de problème, mais au niveau des provinces, il faut travailler avec les autorités provinciales selon l'orientation du programme" (Homme, cadre du système éducatif).

\section{c) Faible application des lois de protection des droits des enfants et du suivi}

L'état des lieux de la législation de la RDC en matière de droits de l'enfant, largement analysé dans le rapport sur l'état des lieux de juillet 2011, permet de faire le constat suivant : le pays dispose d'un arsenal juridique fourni. De même, des avancées ont été enregistrées dans la mise en œuvre de la loi n`09/001 du 10 janvier 2009 portant protection de l'enfant en République Démocratique du Congo. En effet, le décret n`11/01 du 05 janvier 2011 fixe les sièges ordinaires et ressorts des tribunaux pour enfants. De même les arrêtés ministériels $n^{\circ}$ 001/CAB/MIN/Y\&DH/2011 du 05 janvier 2011 portant création des sièges secondaires, des tribunaux pour enfants et fixation de leurs ressorts, et $n^{\circ} 002 / C A B / M I N / Y \& D H / 2011$ du 05 janvier 2011 portant regroupement des ressorts des tribunaux pour enfants pour l'exécution des mesures de garde, d'éducation et de réservation ont été adoptés. II s'agit 
aussi de l'arrêté inter-ministériel $n^{\circ} 490 / C A B / M I N$ Y\& DH/2011 et n011/CAB/MIN GEFAG du 25 décembre 2010 portant composition, organisation et fonctionnement du comité de médiation en matière de Justice pour mineurs ainsi que de l'arrêté ministériel n0248/GC/CAB MIN/AFF SAHSN/09 du 19 novembre 2009 portant réglementation du placement social des enfants en situation difficile.

Mais des difficultés se posent dans l'application des lois. Comme le souligne le Comité sur les droits de l'enfant, en dehors des textes d'application suscités, les lois promulguées ne font pas toujours l'objet de décrets d'application. Ainsi, la plupart des organes de protection sociale ne sont pas fonctionnels et dans le cas où ils le sont, c'est sous l'emprise d'un ancien texte pas toujours favorable à un fonctionnement efficace de la structure. Enfin, les activités entreprises pour faire connaître ces lois sont insuffisantes et par conséquent, ces lois méconnues ne sont pas effectivement appliquées.

\section{d) Distribution inégale des ressources/faible autonomisation des communautés}

L'un des paradoxes congolais évoqué dans la plupart des diagnostics récents dans le domaine du développement, et rappelé dans le Plan intérimaire de l'éducation 2012/2014 (MEPSP, 2012), est le fait que le pays regorge de ressources minières, forestières et agricoles énormes, alors qu'une bonne partie de la population vit dans la pauvreté. Les maux à la base de ce paradoxe sont divers et se résument à une mauvaise et inégale répartition des ressources. A cela s'ajoute une faible capacité des communautés à percevoir et transformer l'ensemble de ces opportunités qui se présentent dans leur environnement. La question a été justement posée aux différents acteurs lors de l'enquête qualitative, de savoir quelles étaient les opportunités de leur territoire en termes de ressources matérielles, financières et institutionnelles qui pourraient aider à relever les défis dans le domaine de l'éducation. De façon générale, il ressort des entretiens que les personnes interviewées ne perçoivent pas les opportunités qu'offre leur environnement. " Notre territoire n'a aucune opportunité pour améliorer notre enseignement, parce que notre territoire n'a pas des sociétés " (Homme, cultivateur, Équateur). Ces propos d'un parent d'élève traduit l'opinion de beaucoup d'acteurs rencontrés. Les opportunités ne sont perçues qu'en termes de sociétés industrielles.

Toutefois, certains acteurs soulignent quelques opportunités de leur environnement. Les ressources forestières, l'agriculture et l'élevage sont citées comme des opportunités dans les provinces du Nord Kivu et de l'Équateur. Au Nord Kivu on cite en plus les ressources du sous-sol telles que les ressources minières, la pêche et les sites touristiques. Dans cette dernière province, la paix et la construction de routes sont mentionnées comme des conditions préalables pour l'utilisation des opportunités de la province.

"Vous avez parlé des ressources naturelles. Ajoutez ressources financières; nous, nous avons notre forêt qui peut être exploité en encadrant par exemple les parents à... donc disons appuyer les initiatives locales. Les ressources naturelles ne manquent pas. La forêt nous fournit du bois, la forêt nous fournit encore les produits agricoles. Puisque nous sommes un territoire à vocation agricole, si on peut encadrer les parents dans ce sens là par des micros crédits, par les projets allant dans le sens des AGR (activités génératrices de revenus) en vendant leurs produits ils peuvent acquérir de l'argent qui peuvent les aider facilement à supporter les frais de scolarisation de leurs enfants " (Homme, cadre du système éducatif, Équateur).

"L'impression que j'ai, c'est que la province du Nord Kivu est une province qui pouvait vivre bien. La grosse difficulté qu'on a ici c'est au niveau de la sécurité... Les possibilités existent parce qu'il y a la richesse minière, il y a une richesse touristique énorme. Moi je suis toujours émerveillé par les sites que je rencontre dans les quelques rares déplacements à l'intérieur. Il y a des endroits très beaux qui pourraient attirer des touristes en masse s'il y avait un minimum de sécurité, de développement. Les possibilités agricoles immenses parce que le terrain ici est fertile, nous sommes à l'équateur, il y a de la pluie et du soleil tous les jours pendant toute l'année, donc il y a des possibilités de pêche, il y a des possibilités d'élevage, donc les possibilités sont énormes. Le Kivu pourrait être une région très riche. S'il ne l'est pas, c'est parce qu'il y a eu ces dernières années la guerre, je crois aussi que l'administration était obligée de faire face à des problèmes urgents et elle a oublié le reste " (Homme, personnel d'une institution, Nord Kivu).

Les propos ci-dessus révèlent les potentialités qui existent dans les provinces qui ont été choisies dans le cadre de l'enquête qualitative. Mais l'ensemble des autres provinces du pays ont également leurs potentialités. Mais encore faut-il que les populations prennent conscience de ces potentialités et 
qu'elles les exploitent pour améliorer leurs conditions de vie et relever les défis de l'éducation. Une sensibilisation et un accompagnement est sans doute nécessaire dans ce sens.

\section{e) Faiblesse du système de protection sociale}

L'analyse secondaire des données d'enquêtes auprès des ménages, faite dans l'état des lieux montre que le niveau de protection sociale des enfants et de leurs ménages est très faible, révélant ainsi le fossé qui existe entre l'existence des textes et des cadres juridiques et leur effectivité. En effet, seulement un enfant de moins de 5 ans sur cinq $(20,7 \%)$ possédait un acte de naissance en 2010. Par ailleurs, il ressort qu'au cours des douze derniers mois précédant l'enquête MICS 2010, la proportion d'enfants de 0 à 17 ans qui avaient reçu des soutiens était très faible: $3,1 \%$ pour le soutien médical, 1,0 \% pour le soutien social, 5,5\% pour le soutien scolaire, 1,8\% pour le soutien matériel et $7,3 \%$ pour le soutien émotionnel. L'étude diagnostique sur la " protection sociale adaptée aux besoins des enfants en RDC » réalisée en 2011 par Overseas Development Institute (ODI), nous donne une bonne synthèse de la situation et des défis de la mise en place d'un système de protection sociale performante en RDC (Bailey et al., 2011). Le Document de stratégie pour la réduction de la pauvreté (DSRP) de la RDC pour la période 2006-2008 souligne l'absence d'une politique nationale claire en matière de protection sociale. II mentionne également le fait qu'il y a de nombreuses interventions provenant de plusieurs intervenants (Etat, ONG, communautés de base, Eglises), mais qui malheureusement ne sont pas coordonnées et donc ne sont pas stratégiques. Par ailleurs, ces actions ont des portées limitées puisque ne touchant qu'une faible frange de la population. Devant cette absence de mécanismes étatiques formels de protection sociale, les populations s'appuient donc sur une série de réseaux informels de filets de sécurité sociale, pas toujours efficaces (Bailey et al., 2011).

\section{Résumé analytique}

L'objectif de ce chapitre était de dégager les barrières et goulots d'étranglement de l'éducation. II s'agissait donc de répondre à la question de recherche " pourquoi les enfants et adolescents sont-ils en dehors de l'école? " Recourant à des méthodes d'analyse statistique multivariée pour ce qui est des données quantitatives et une analyse des discours pour ce qui est des données qualitatives, l'étude a aboutit à une identification des déterminants de l'exclusion. Ceux-ci se situent au niveau de la demande, de l'offre et du politique.

Les déterminants de l'exclusion scolaire au niveau de la demande sont socioculturels et économiques. Au niveau socioculturel, les écarts entre les sexes sont encore importants au niveau du secondaire, particulièrement en milieu rural. Les représentations sociales sur les rôles et statuts de chaque sexe, notamment les logiques matrimoniales, entraînent souvent des choix familiaux d'investissement scolaire en défaveur des filles. Le décès des parents accroît aussi considérablement les risques des enfants et des adolescents d'être en dehors de l'école. Le confiage des enfants est aussi apparu au niveau socioculturel comme un des déterminants de l'exclusion scolaire, dans la mesure où les enfants confiés sont souvent utilisés pour accomplir de multiples tâches dans leur ménage d'accueil, diminuant ainsi leurs chances de participation scolaire.

Par ailleurs, les résultats des analyses quantitatives montrent que, quel que soit le milieu de résidence, le niveau d'instruction du chef de ménage est l'un des principaux déterminants de l'exclusion scolaire. Les enfants vivant dans les ménages où le chef a le niveau d'instruction secondaire ou plus, ont moins de risque de se retrouver en dehors de l'école que ceux dont le chef de ménage n'a aucun niveau d'instruction.

Au niveau économique, le revenu du ménage est apparu, quel que soit le milieu de résidence, comme un des déterminants majeurs de la fréquentation scolaire des enfants et des adolescents. La barrière financière est ainsi la principale raison évoquée par les ménages pour justifier la non inscription à l'école ou l'abandon scolaire des enfants, ce qui est aussi confirmée par les propos des acteurs interviewés lors des entretiens qualitatifs.

Au niveau de l'offre scolaire, la principale barrière demeure les longues distances que les enfants et adolescents sont amenées à parcourir, posant ainsi le problème de la disponibilité des infrastructures scolaires, de l'équipement et du personnel enseignant. Bien qu'on note globalement une augmentation de l'offre scolaire ces dernières années à tous les niveaux du système éducatif, il existe 
des disparités entre provinces et entre milieux urbain et rural, et le problème se pose particulièrement en milieu rural.

En ce qui concerne les barrières relevant du niveau politique, il ressort la faiblesse du financement de l'Etat, qui contraint les ménages à consacrer une part importante de leur revenu annuel aux dépenses d'éducation, mais aussi la faiblesse de la capacité institutionnelle et humaine du secteur de l'éducation. Ce problème de capacité institutionnelle et humaine se pose au niveau du fonctionnement des comités de gestion des écoles, au niveau de la gestion des ressources humaines et au niveau de la coordination et de la gestion du système éducatif. Globalement les acteurs soulignent un disfonctionnement des comités de gestion (faible capacité de gestion, manque de concertation et de transparence dans la gestion des fonds), et une faible communication entre les différents acteurs.

En résumé, les barrières à la scolarisation des enfants et adolescents sont énormes et relèvent de divers domaines. On ne peut à priori privilégier une dimension à une autre. Pour lever ces barrières et accroître quantitativement et qualitativement la participation des enfants à l'école, il faut une approche holistique des politiques et stratégies. 


\section{Politiques et stratégies}

Ce dernier chapitre se propose, à la lueur des résultats obtenus à travers l'état des lieux, mais aussi des enseignements de l'enquête nationale sur les EADE (volet quantitatif comme volet qualitatif), notamment les conclusions du chapitre sur les barrières et les goulots d'étranglement, de faire quelques suggestions en termes de politiques ou de stratégies qui pourront guider les décideurs en vue d'actions ciblées pour ramener tous les enfants et adolescents à l'école. Les propositions d'actions qui sont faites s'inspirent également des comptes rendus des missions de présentation de la première version du rapport aux acteurs au niveau provincial.

L'examen des documents de politique récents, notamment le Plan intérimaire de l'éducation 2012/2014, la Politique nationale sur les orphelins et enfants vulnérables, le document de diagnostic réalisé par Overseas Development Institue (ODI) sur la protection sociale des enfants en RDC, présentent déjà un certain nombre d'axes stratégiques en vue d'améliorer l'accès et le maintien à l'école des enfants et adolescents en RDC. L'étude EADE-RDC vient donner les éclairages chiffrés et les discours issus des entretiens qualitatifs, qui renforcent les orientations stratégiques opérées. Le rapport fait en outre des suggestions sur la base des expériences passées ou expérimentées dans d'autres contextes.

\subsection{Standards internationaux pour répondre aux 5 dimensions de l'exclusion}

Avant de présenter les politiques et stratégies propres au contexte de la RDC, il convient d'avoir une vue d'ensemble de ce qui apparaît selon les expériences au niveau international, comme les principales politiques permettant de résoudre le problème des enfants et adolescents en dehors de l'école. Selon le Rapport mondial de suivi de l'éducation pour tous 2010, " II n'y a pas de formule miracle pour surmonter la marginalisation dans l'éducation. II faut que les politiques s'attaquent aux causes profondes telles que l'inégalité sociale, les disparités entre les sexes, les désavantages ethniques et linguistiques et les écarts entre les zones géographiques. Dans chacun de ces domaines, l'égalisation des chances exige que l'on remédie à des rapports de pouvoir inégaux. Les inégalités auxquelles sont confrontés les marginalisés commencent durant la petite enfance et continuent pendant toute la scolarité. Elles sont particulièrement tenaces et très résistantes au changement. Pourtant, des progrès sont possibles avec un engagement politique soutenu en faveur de la justice sociale, de l'égalité des chances et des droits fondamentaux. »(UNESCO, 2010, p. 13).

Comme le montre le tableau 42, le rapport mondial de suivi de l'éducation 2010 a identifié trois grandes catégories de politiques qui peuvent faire une différence énorme pour tout pays voulant s'attaquer à la question de l'accès et de l'achèvement dans l'éducation de base. Ce sont : des politiques de promotion de l'accès, des politiques de promotion de l'environnement d'apprentissage et des politiques qui assurent les droits et les opportunités élémentaires pour les pauvres et les marginalisés.

Tableau 42 : Principales politiques pour répondre aux défis des EADE

\begin{tabular}{|c|c|c|}
\hline $\begin{array}{l}\text { Politiques de promotion de } \\
\text { l'accessibilité }\end{array}$ & $\begin{array}{l}\text { Politiques de promotion } \\
\text { l'environnement d'apprentissage }\end{array}$ & $\begin{array}{l}\text { Politiques assurant les droits } \\
\text { et opportunités }\end{array}$ \\
\hline $\begin{array}{ll}\text { - } & \text { Réduire les coûts directs et } \\
\text { indirects } \\
\text { - } \\
\text { Octroyer des compensations } \\
\text { financières ciblées } \\
\text { - Investir dans les infrastructures } \\
\text { - } \quad \text { Rapolaires } \\
\text { Rapprocher les classes des } \\
\text { enfants } \\
\text { - Soutenir les appuis flexibles } \\
\text { - } \text { noordonner et suivre les appuis } \\
\end{array}$ & $\begin{array}{l}\text { - } \text { Répartir les enseignants de } \\
\text { manière équitable } \\
\text { - } \quad \text { Recruter et former les } \\
\text { enseignants des groupes } \\
\text { marginalisés } \\
\text { - } \quad \text { Procurer un soutien additionnel } \\
\text { aux écoles défavorisées } \\
\text { - Elaborer un curriculum pertinent } \\
\text { - Faciliter l'éducation } \\
\text { interculturelle et bilingue }\end{array}$ & 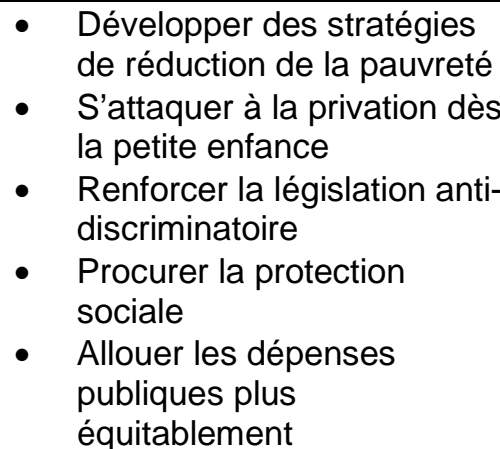 \\
\hline
\end{tabular}

Source: Synthèse faite à travers le rapport mondial de suivi de l'EPT (UNESCO, 2010) et Ghana country study (Government of Ghana and UNICEF, 2012). 


\subsection{L'éducative inclusive dans les documents de politique en RDC}

Le Document de Stratégie de développement du sous-secteur de l'enseignement primaire, secondaire et professionnel 2010/2011-2015/2016 adopté en mars 2010 se fixe comme objectif général de " construire un système éducatif inclusif et de qualité ". Cet objectif de la stratégie de développement de l'EPSP s'inscrit dans l'esprit de la loi fondamentale du pays qu'est la Constitution, qui consacre la gratuité et le caractère obligatoire de l'enseignement primaire. Principe qui va par la suite conduire au lancement de la politique de gratuité scolaire au niveau des quatre premières années du primaire en 2010. Mais comme cela ressort de l'étude EADE 2012, l'effectivité de la politique de gratuité se heurte à de nombreux défis, et il faudra certainement un engagement plus ferme de l'ensemble des acteurs et des mécanismes de suivi en vue de son effectivité, mais aussi son extension à l'ensemble du cycle primaire comme suggéré par les personnes interviewées lors de l'enquête EADE-RDC.

Le Plan intérimaire de l'éducation 2012/2014 comporte un certain nombre d'axes stratégiques ou de programmes tendant à cibler la question des EADE. On peut mentionner :

- L'appui aux communautés locales pour le développement de l'éducation préscolaire: vu l'importance de la préparation de la petite enfance pour la suite du parcours scolaire, l'objectif visé dans ce sous-programme est de développer en collaboration avec les communautés, d'autres modèles d'institutions du préscolaire plus accessibles au plus grand nombre d'enfants ;

- En matière d'universalisation progressive de l'enseignement primaire, il est prévu la prise en charge des frais scolaires par l'Etat, la question de l'insertion des enfants en dehors de l'école, le soutien à la scolarisation des filles ;

- Le renforcement des capacités d'accueil du système éducatif, notamment en réduisant la distance à l'école, mais aussi en accordant une attention particulière aux enfants vivants avec un handicap.

\subsection{Politiques et stratégies du côté de la demande socio- culturelle}

Sur la base des barrières socio-culturelles relevées dans le chapitre 3 , le tableau 43 donne un aperçu pour chaque type de barrières, des stratégies ou actions possibles à entreprendre. 
Tableau 43 : Politiques et stratégies pour répondre aux barrières socio-culturelles

\begin{tabular}{|c|c|c|c|}
\hline $\begin{array}{l}\text { Barrières socio- } \\
\text { cultuelles }\end{array}$ & Stratégies ou actions & Expériences réussies & Impact sur l'éducation \\
\hline $\begin{array}{l}\text { Perceptions } \\
\text { négatives ou } \\
\text { normes sociales } \\
\text { discriminatoires } \\
\text { envers l'éducation } \\
\text { des filles }\end{array}$ & $\begin{array}{l}\text { Mettre en place des stratégies orientées } \\
\text { vers l'accroissement de la scolarisation } \\
\text { des filles, particulièrement au niveau du } \\
\text { secondaire (campagnes de } \\
\text { sensibilisation, programme d'octroi de } \\
\text { bourses, dotations incitatives aux } \\
\text { ménages...) }\end{array}$ & $\begin{array}{l}\text { Exemples: } \\
\text {-Bangladesh : programme } \\
\text { d'assistance aux filles de } \\
\text { l'enseignement secondaire, } \\
\text { dans le cadre du } \\
\text { Programme national } \\
\text { d'allocation de } \\
\text { bourses aux filles (1994-); } \\
\text { - Burkina Faso : BRIGHT } \\
\text { (Burkinabe Response to } \\
\text { Improve girls'chances To } \\
\text { succeed/Réponse } \\
\text { burkinabé pour améliorer } \\
\text { les chances de réussite } \\
\text { des filles) (2005-); } \\
\text {-Pakistan: Programme de } \\
\text { réforme du secteur éducatif } \\
\text { du Penjab (2003-) }\end{array}$ & $\begin{array}{l}\text { Exemples: } \\
\text { Bangladesh } \\
\text { accroissement de } 12 \text { points } \\
\text { de pourcentage de la } \\
\text { scolarisation } \\
\text { Burkina Faso } \\
\text { accroissement de } 20 \text { points } \\
\text { de pourcentage de la } \\
\text { scolarisation, } \\
\text { accroissement de } 16 \\
\text { points de pourcentage de } \\
\text { l'assiduité, accroissement } \\
\text { de } 0,4 \text { écart type des } \\
\text { résultats aux tests de } \\
\text { mathématiques et de } \\
\text { français (ce qui équivaut à } \\
\text { une progression } \\
\text { du } 50 \text { au } 80 \text { percentile) } \\
\text {-Pakistan : accroissement } \\
\text { de } 11,1 \quad \% \\
\text { scolarisation de la }\end{array}$ \\
\hline $\begin{array}{l}\text { Faible prise de } \\
\text { conscience des } \\
\text { droits des enfants et } \\
\text { l'analphabétisme } \\
\text { des parents }\end{array}$ & $\begin{array}{l}\text { - Mettre en place des campagnes de } \\
\text { sensibilisation orientées vers les chefs } \\
\text { de ménage non instruits/non } \\
\text { alphabétisés } \\
\text { - Renforcer les programmes } \\
\text { d'alphabétisation, les capacités socio- } \\
\text { économiques des jeunes et des adultes, } \\
\text { et augmenter la connaissance des } \\
\text { acteurs des lois relatives à la protection } \\
\text { et au droit de l'enfant }\end{array}$ & $\begin{array}{l}\text { Exemple: } \\
\text {-Bouthan : Programme } \\
\text { d'éducation non formelle et } \\
\text { de formation continue }\end{array}$ & $\begin{array}{l}\text { Exemple: } \\
\text {-Bouthan : } \\
\text { d'alphabétisation taux } \\
\text { adultes de } 70 \% \text { d'ici à } \\
2013\end{array}$ \\
\hline $\begin{array}{l}\text { Mauvais confiage } \\
\text { des enfants }\end{array}$ & $\begin{array}{l}\text { Développer les programmes d'éducation } \\
\text { parentale afin de rappeler aux parents } \\
\text { leurs obligations envers les enfants et } \\
\text { promouvoir le soutien parental aux } \\
\text { enfants, que ceux-ci soient à l'intérieur } \\
\text { de l'unité familiale ou en dehors }\end{array}$ & $\begin{array}{l}\text { Exemple: } \\
\text {-Cambodge : Programme } \\
\text { communautaire d'éducation } \\
\text { parentale et maternelle en } \\
\text { zone rurale }\end{array}$ & $\begin{array}{l}\text { Exemple: } \\
\text {-Cambodge : } 3600 \text { familles } \\
\text { dont } 1845 \text { enfants de } 3 \text { à } 5 \\
\text { ans }\end{array}$ \\
\hline Mariages précoces & $\begin{array}{l}\text { Renforcer les lois sur le droit des enfants } \\
\text { et des adolescents, notamment l'âge } \\
\text { légal au mariage }\end{array}$ & & \\
\hline $\begin{array}{l}\text { Grossesses des } \\
\text { adolescentes }\end{array}$ & $\begin{array}{l}\text { Renforcer l'éducation en matière de } \\
\text { santé de la reproduction dans les } \\
\text { établissements, notamment à travers } \\
\text { des cours formels, mais aussi l'éducation } \\
\text { par les pairs, l'implication des } \\
\text { organisations de la société civile }\end{array}$ & & \\
\hline $\begin{array}{l}\text { Faible prise de } \\
\text { conscience des } \\
\text { communautés des } \\
\text { potentialités } \\
\text { économiques de } \\
\text { leur environnement }\end{array}$ & $\begin{array}{l}\text { Mettre en place des approches } \\
\text { multisectorielles pour renforcer les } \\
\text { capacités institutionnelles et } \\
\text { communautaires d'autonomisation en } \\
\text { matière de mobilisation de ressources } \\
\text { financières pour amener les populations } \\
\text { vulnérables à réaliser leurs potentialités } \\
\text { économiques dans leur environnement } \\
\text { immédiat. }\end{array}$ & & \\
\hline
\end{tabular}

Source : Exemples tirés du rapport mondial de suivi de l'EPT 2010 (UNESCO, 2010) 


\subsection{Politiques du côté de la demande économique}

En ce qui concerne la demande économique, l'état des lieux comme le rapport actuel ont bien montré combien la pauvreté (faiblesse des revenus des ménages) était un des facteurs les plus déterminants de risque pour les enfants et adolescents d'être en dehors de l'école. C'est aussi sans doute ce facteur de pauvreté qui explique aussi la forte participation des enfants au travail. Le tableau 44 synthétise ces barrières ainsi que les stratégies pour y répondre.

Tableau 44 : Politiques et stratégies pour répondre aux barrières économiques

\begin{tabular}{|c|c|c|c|}
\hline $\begin{array}{l}\text { Barrières } \\
\text { économiques }\end{array}$ & Stratégies ou actions & Expériences réussies & Impact sur l'éducation \\
\hline $\begin{array}{l}\text { Coûts directs de la } \\
\text { scolarisation } \\
\text { (Pauvreté) }\end{array}$ & $\begin{array}{l}\text { - Accélérer le processus de } \\
\text { réduction des frais scolaires } \\
\text { - Généraliser la gratuité } \\
\text { scolaire à l'ensemble du cycle } \\
\text { primaire } \\
\text { - Mettre en place des } \\
\text { systèmes de dotation } \\
\text { financière ou d'octroi de } \\
\text { bourses pour les plus démunis }\end{array}$ & $\begin{array}{l}\text { Exemples: } \\
\text {-Paraguay : Ménages des } \\
\text { quartiers les plus pauvres } \\
\text { ayant un faible indicateur de } \\
\text { qualité de vie et des enfants } \\
\text { âgés de } 15 \text { ans ou moins; } \\
\\
\text { - Nicaragua : Red de } \\
\text { protección social (2000- } \\
2005 / 6)\end{array}$ & $\begin{array}{l}\text { Exemples : } \\
\text {-Paraguay : accroissement } \\
\text { de 5-8 points de } \\
\text { pourcentage de l'assiduité, } \\
\text { en particulier chez les } \\
\text { garçons et les enfants plus } \\
\text { âgés; } \\
\text { Nicaragua : accroissement } \\
\text { de } 12,8 \text { de points de } \\
\text { pourcentage de la } \\
\text { scolarisation (25 points de } \\
\text { pourcentage chez les plus } \\
\text { pauvres), accroissement de } \\
7 \text { points du pourcentage } \\
\text { d'élèves progressant de } 2 \\
\text { classes en } 2 \text { ans }\end{array}$ \\
\hline $\begin{array}{l}\text { Coûts indirects ou } \\
\text { coûts d'opportunité } \\
\text { de la scolarisation } \\
\text { (Pauvreté) }\end{array}$ & $\begin{array}{l}\text { - Renforcer et généraliser les } \\
\text { systèmes de micro-crédits aux } \\
\text { femmes en lien avec } \\
\text { l'éducation des filles et celle } \\
\text { des autres EADE } \\
\text { - Généraliser les systèmes de } \\
\text { cantines scolaires }\end{array}$ & $\begin{array}{l}\text { Exemples: } \\
\text {-Burkina Faso: Cantine } \\
\text { scolaire (programmes de } \\
2005-2006 \text { ) }\end{array}$ & $\begin{array}{l}\text { Exemples: } \\
\text {-Burkina Faso : } \\
\text { accroissement de 5-6 points } \\
\text { de pourcentage de la } \\
\text { scolarisation des filles les } \\
\text { plus jeunes, baisse de } \\
\text { l'absentéisme des filles }\end{array}$ \\
\hline Travail des enfants & $\begin{array}{l}\text { - Renforcer les lois sur la } \\
\text { protection et les droits des } \\
\text { enfants et adolescents } \\
\text { - Renforcer les programmes } \\
\text { d'éducation parentale }\end{array}$ & & \\
\hline
\end{tabular}

Source : Exemples tirés du rapport mondial de suivi de l'EPT 2010 (UNESCO, 2010)

\subsection{Politiques du côté de l'offre}

L'importance de la distance à l'école, particulièrement en milieu rural et dans plusieurs provinces, comme facteur de risque d'être en dehors de l'école, mais aussi l'importance des entrées tardives à l'école, révèlent bien la faiblesse de l'offre scolaire. Par ailleurs la proportion importante d'enfants et adolescents vivant avec un handicap en dehors de l'école, montre la nécessité de mettre en place une offre adéquate (éducation inclusive) pour cette population d'enfants. Enfin, la nécessité de former l'ensemble des acteurs et de sensibiliser davantage sur les lois portant sur les droits des enfants (tableau 45). 
Tableau 45 : Politiques et stratégies pour répondre aux barrières liées à l'offre

\begin{tabular}{|c|c|c|c|}
\hline Barrières liées à & Stratégies ou actions & Expériences réussies & Impact sur l'éducation \\
\hline $\begin{array}{l}\text { Manque et inégale } \\
\text { distribution des } \\
\text { infrastructures } \\
\text { entraînant des } \\
\text { longues distances } \\
\text { à parcourir et une } \\
\text { entrée tardive à } \\
\text { l'école }\end{array}$ & $\begin{array}{l}\text { Accroître l'offre } \\
\text { fonction de la calaire en } \\
\text { nationale et des cartes scolaire } \\
\text { provinciales pour respecter le } \\
\text { critère du rayon maximum pour } \\
\text { une école; }\end{array}$ & $\begin{array}{lr}\text { Exemple : } & \\
& \\
\text {-Burkina Faso : } & \text { Plan } \\
\text { Décennal } & \text { de } \\
\text { Développement } & \text { de } \\
\text { l'Education de Base } & \end{array}$ & $\begin{array}{l}\text { Exemple: } \\
\text {-Burkina Faso : nombre } \\
\text { d'écoles est passé de } \\
4860 \text { en } 2000 \text { à } 10198 \text { en } \\
2009-2010 \text { soit une } \\
\text { augmentation de } 200 \% \text { en } \\
\text { dix ans }\end{array}$ \\
\hline $\begin{array}{lr}\text { Manque } & \text { de } \\
\text { formation } & \text { ou } \\
\text { formation limitée } \\
\text { des enseignants }\end{array}$ & $\begin{array}{l}\text { - Accroître en nombre et en } \\
\text { qualité le personnel enseignant, } \\
\text { (surtout la proportion des femmes } \\
\text { enseignantes pour accroître la } \\
\text { rétention des filles à l'école). } \\
\text { - Améliorer de manière } \\
\text { substantielle la rémunération des } \\
\text { enseignants tout en leur assurant } \\
\text { un plan de carrière adéquat }\end{array}$ & $\begin{array}{l}\text { Exemple: } \\
\text {-Burkina Faso : Plan } \\
\text { Décennal de } \\
\text { Développement de } \\
\text { l'Education de Base }\end{array}$ & \\
\hline $\begin{array}{l}\text { Inadaptation } r \text { du } \\
\text { système éducatif } \\
\text { aux diverses } \\
\text { situations de } \\
\text { vulnérabilité } \\
\text { (enfants vivant } \\
\text { avec un handicap ; } \\
\text { enfants travailleurs, } \\
\text { enfants de la rue) }\end{array}$ & $\begin{array}{l}\text { - Mettre en place des approches } \\
\text { pédagogiques spécifiques pour } \\
\text { chaque catégorie d'enfant vivant } \\
\text { avec un handicap } \\
\text { - Renforcer les initiatives tendant } \\
\text { à rendre possible la scolarisation } \\
\text { des enfants de la rue } \\
\text { - Renforcer les initiatives tendant } \\
\text { à permettre la scolarisation des } \\
\text { enfants dans les sites d'orpaillage }\end{array}$ & & \\
\hline $\begin{array}{l}\text { Faible } \\
\text { appropriation des } \\
\text { lois portant } \\
\text { protection et droit } \\
\text { des enfants }\end{array}$ & $\begin{array}{l}\text { Mettre en place des programmes } \\
\text { de renforcementrdes des } \\
\text { compétences des acteurs tant à } \\
\text { l'intérieur du système éducatif } \\
\text { qu'en dehors, sur les droits des } \\
\text { enfants }\end{array}$ & & \\
\hline
\end{tabular}

Source : Exemples tirés du rapport mondial de suivi de l'EPT 2010 (UNESCO, 2010)

\subsection{Au niveau politique, de la gestion et de la gouvernance}

L'analyse des dépenses d'éducation des ménages et leur mise en rapport avec le budget national alloué à l'EPSP, ainsi que les résultats des entretiens qualitatifs ont fait ressortir un certain nombre d'obstacles limitant l'efficacité des actions dans le secteur de l'éducation, en lien avec la question du financement, de la gestion et de la gouvernance. 
Tableau 46 : Politiques et stratégies pour répondre aux barrières liées à la gestion et la gouvernance

\begin{tabular}{|c|c|c|c|}
\hline $\begin{array}{l}\text { Barrières } \\
\text { politiques }\end{array}$ & Stratégies ou actions & Expériences réussies & Impact sur l'éducation \\
\hline $\begin{array}{l}\text { Faible financement } \\
\text { de l'Etat dans le } \\
\text { secteur } \\
\text { l'éducation }\end{array}$ & $\begin{array}{l}\text { - Accroître de manière } \\
\text { substantielle les ressources de } \\
\text { l'Etat allouées à l'éducation, } \\
\text { notamment le financement aux } \\
\text { établissements en vue de réduire } \\
\text { le poids des frais scolaires pour } \\
\text { les familles } \\
-\quad \text { Développer un partenariat } \\
\text { Etat/privé, avec des fora annuels } \\
\text { en vue de prévenir l'exploitation } \\
\text { économique des enfants et des } \\
\text { jeunes } \\
-\quad \text { Instituer un « fonds de } \\
\text { compensation communautaire " } \\
\text { dans les zones à forte production } \\
\text { industrielle en vue de financer les } \\
\text { investissements scolaires et } \\
\text { l'appui aux groupes vulnérables } \\
\text { - Etudier les options possibles } \\
\text { pour une augmentation } \\
\text { progressive de la participation à } \\
\text { l'enseignement secondaire pour } \\
\text { les filles et les garçons. }\end{array}$ & $\begin{array}{l}\text { Exemple : } \\
\text {-Turquie : Projet de } \\
\text { réduction } \\
\text { du risque social (2002-) }\end{array}$ & $\begin{array}{l}\text { Exemple : } \\
\text {-Turquie : Élèves du } \\
\text { primaire : accroissement } \\
\text { de 3 points du } \\
\text { pourcentage de la } \\
\text { scolarisation } \\
\text { Élèves du secondaire : } \\
\text { pas d'augmentation } \\
\text { significative de la } \\
\text { scolarisation }\end{array}$ \\
\hline 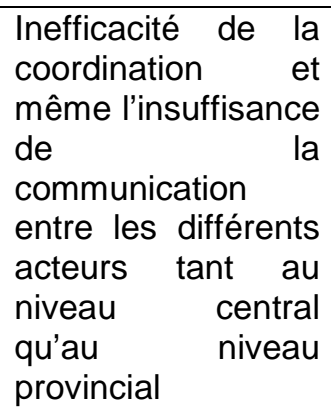 & $\begin{array}{l}\text { - Améliorer le système de pilotage } \\
\text { (communication entre acteurs au } \\
\text { niveau central mais aussi entre } \\
\text { niveau central et niveau local) } \\
\text { - Assurer une large diffusion des } \\
\text { annuaires statistiques au niveau } \\
\text { national }\end{array}$ & & \\
\hline $\begin{array}{l}\text { Distribution inégale } \\
\text { des } \\
\text { ressources/faible } \\
\text { autonomisation des } \\
\text { communautés }\end{array}$ & $\begin{array}{l}\text { - Mettre en place des } \\
\text { mécanismes au niveau provincial } \\
\text { en vue d'une sensibilisation et } \\
\text { d'une information sur les } \\
\text { potentialités économiques et } \\
\text { intellectuelles des provinces }\end{array}$ & & \\
\hline
\end{tabular}

Source : Exemples tirés du rapport mondial de suivi de l'EPT 2010 (UNESCO, 2010)

\subsection{Protection sociale}

L'une des conclusions majeures à laquelle aboutit l'étude sur la protection sociale citée précédemment (Bailey et al., 2011) est l'absence de mécanismes formels de protection sociale dans le pays, amenant les populations à développer leurs propres stratégies de protection à travers divers réseaux et mécanismes informels, qui peuvent dans certains cas être préjudiciables pour les populations. II existe plusieurs initiatives développées par-ci par-là par des organisations de la société civile, les organisations internationales, en collaboration avec l'Etat, mais elles restent de portée très limitée, notamment du fait de la faiblesse des ressources qui y sont allouées. L'étude arrive à un certain nombre de recommandations, notamment en ce qui concerne quelques expériences prometteuses qui pourraient être généralisées. Mais il s'agit dans la plupart des cas d'expériences cherchant à réduire la vulnérabilité des ménages et ne sont pas uniquement orientées vers l'amélioration de la participation scolaire. Par ailleurs, le rapport mondial pour le suivi de l'éducation de 
2010, a fait une synthèse de quelques expériences à travers le monde des effets sur l'éducation de certains programmes de protection sociale (UNESCO, 2010). Nous en reprenons quelques unes cidessous (tableau 47).

Tableau 47 : Quelques stratégies ou actions visant les groupes vulnérables

\begin{tabular}{|c|c|c|c|}
\hline $\begin{array}{l}\text { Groupes } \\
\text { vulnérables }\end{array}$ & Stratégies ou actions & Expériences réussies & Impact sur l'éducation* \\
\hline $\begin{array}{l}\text { Enfants et } \\
\text { adolescents issus } \\
\text { des } \\
\text { familles/ménages } \\
\text { les plus pauvres }\end{array}$ & $\begin{array}{l}\text { - Transferts monétaires aux } \\
\text { ménages les plus pauvres, } \\
\text { conditionnés à la participation } \\
\text { scolaire et à l'assiduité } \\
\text { - Octroi de petites bourses } \\
\text { d'étude } \\
\text { - Cantines scolaires dans les } \\
\text { écoles, notamment en milieu rural } \\
\text { - Rations alimentaires à emporter } \\
\text { en famille (par exemple pour les } \\
\text { filles de certaines classes } \\
\text { données) }\end{array}$ & $\begin{array}{l}\text { Exemple : } \\
\text { - Ethiopie : Programme } \\
\text { Filet de sécurité productif } \\
\text { (2005) }\end{array}$ & $\begin{array}{l}\text { Exemple : } \\
\text { Ethiopie : accroissement } \\
\text { de 19-23 points du } \\
\text { pourcentage de l'assiduité } \\
\text { chez les garçons, } \\
\text { accroissement de la } \\
\text { scolarisation dans environ } \\
33 \% \text { des ménages, } \\
\text { accroissement de mois de } \\
\text { scolarité dans environ } 50 \\
\% \text { des ménages, } \\
\text { accroissement du temps } \\
\text { d'étude à la maison }\end{array}$ \\
\hline Orphelins & $\begin{array}{l}\text { Octroyer des subventions aux } \\
\text { ménages extrêmement pauvres } \\
\text { accueillant des enfants et } \\
\text { adolescents orphelins }\end{array}$ & $\begin{array}{l}\text { Exemple: } \\
-\quad \text { Kenya : Ménages } \\
\text { extrêmement pauvres } \\
\text { accueillant un orphelin ou } \\
\text { un enfant vulnérable âgé } \\
\text { de } 17 \text { ans ou moins et ne } \\
\text { bénéficiant d'aucune aide; } \\
\text { enfant pris en charge par } \\
\text { un adulte atteint d'une } \\
\text { maladie chronique }\end{array}$ & $\begin{array}{l}\text { Exemple: } \\
\text {-Kenya : Évaluation } \\
\text { définitive non disponible } \\
\text { mais on a constaté une } \\
\text { amélioration de l'assiduité } \\
\text { et de la rétention. Quatre } \\
\text { projets pilotes } \\
\text { actuellement financés } \\
\text { pour une mise à l'échelle } \\
\text { en tant que programme } \\
\text { ordinaire }\end{array}$ \\
\hline Enfants de la rue & $\begin{array}{l}\text { Renforcer le partenariat } \\
\text { Etat/organisation de la société } \\
\text { civile : } \\
\text { - Pour une sensibilisation et une } \\
\text { éducation parentale plus accrues } \\
\text { sur les rapports parent/enfant } \\
\text { - Pour accroître les capacités } \\
\text { d'accueil des structures d'accueil }\end{array}$ & & \\
\hline Enfants travailleurs & $\begin{array}{l}\text { - Une sensibilisation et une } \\
\text { éducation parentale plus accrues } \\
\text { sur les rapports parent/enfant } \\
\text { - Intensifier les initiatives } \\
\text { entreprises en collaboration avec } \\
\text { le BIT à travers le programme } \\
\text { IPEC pour l'abolition des activités } \\
\text { dangereuses pour les enfants } \\
\text { - Rendre effective l'application de } \\
\text { la législation sur la protection de } \\
\text { l'enfant; } \\
\text { - Dans les zones minières, } \\
\text { entreprendre des activités de } \\
\text { formation à d'autres métiers pour } \\
\text { les jeunes EADE dans les mines. }\end{array}$ & & \\
\hline
\end{tabular}

Source : Exemples tirés du rapport mondial de suivi de l'EPT 2010 (UNESCO, 2010) 
En plus de ces actions ciblant des groupes vulnérables, on peut également envisager des activités de nature transversale, dont :

- La vulgarisation des lois promulguées sur les droits des enfants, notamment en produisant des dépliants en langues nationales ;

- L'introduction de formations pour l'ensemble des acteurs sur la consolidation de la paix et la promotion des droits de l'enfant.

\section{Résumé analytique}

Quelles politiques et quelles stratégies pour subjuguer les différentes barrières à la participation scolaire des enfants et adolescents en RDC ? Les politiques et stratégies proposées sont structurées autour du même regroupement que les barrières et goulots d'étranglements en quatre catégories (socio-culturelles, économiques, de l'offre, et politiques) auxquelles nous avons ajouté des éléments sur la protection sociale.

Au niveau socio-culturel, un certain nombre de stratégies ou d'actions ont été proposées pour répondre aux problèmes des représentations sociales défavorables aux filles, aux mariages et grossesses précoces, aux perceptions sur les droits et la place de l'enfant dans la société congolaise, aux effets parfois néfastes du confiage des enfants et aux perceptions des communautés sur les potentialités économiques de leur environnement de vie. En ce qui concerne la scolarisation des filles, il est proposé d'intensifier les campagnes de sensibilisation, pour la scolarisation des filles, particulièrement au niveau du secondaire, de mettre en place des programmes d'octroi de bourses et envisager comme cela a été expérimenté ailleurs, des dotations incitatives aux ménages. Pour éliminer le phénomène des mariages précoces, il est suggéré de faire appliquer la législation en matière d'âge légal au mariage et pour les grossesses précoces, d'intensifier les cours d'éducation en santé sexuelle et reproductive.

En ce qui concerne le faible niveau d'instruction et d'alphabétisation des parents et des perceptions sur les droits des enfants, il est suggéré :

- De mettre en place des campagnes de sensibilisation orientées vers les chefs de ménage non instruits/non alphabétisés ;

- De renforcer les programmes d'alphabétisation, les capacités socio-économiques des jeunes et des adultes, et augmenter la connaissance des acteurs sur les lois relatives à la protection et au droit de l'enfant.

Quant aux effets parfois néfastes du confiage, il s'agira de développer les programmes d'éducation parentale afin de rappeler aux parents leurs obligations envers leurs enfants. Enfin, pour accroître les capacités et les ressources des communautés, il est proposé de mettre en place des approches multisectorielles pour renforcer les capacités institutionnelles et communautaires d'autonomisation en matière de mobilisation de ressources financières pour permettre aux populations vulnérables de tirer profit des potentialités économiques dans leur environnement immédiat.

Au niveau des barrières économiques, le rôle important joué par la pauvreté comme barrière à la scolarisation des enfants conduit à réaffirmer l'urgence qu'il y a d'accélérer et de rendre effective la réduction des frais scolaires. En ce qui concerne le travail des enfants, particulièrement le travail dangereux, il s'agira de renforcer les lois sur la protection et les droits des enfants et adolescents, et renforcer les programmes d'éducation parentale.

Au niveau de l'offre, il s'agira d'accroître substantiellement les infrastructures, accroître en nombre et en qualité le personnel enseignant (particulièrement la proportion des femmes enseignantes pour accroître la rétention des filles à l'école), améliorer de manière substantielle la rémunération des enseignants tout en leur assurant un plan de carrière adéquat, mettre en place des approches pédagogiques spécifiques pour chaque catégorie d'enfant vivant avec un handicap, renforcer les initiatives tendant à rendre possible la scolarisation des enfants de la rue et renforcer les initiatives tendant à permettre la scolarisation des enfants dans les sites d'orpaillage.

Au plan politique et de gouvernance, il est proposé :

- D'accroître de manière substantielle les ressources de l'Etat allouées à l'éducation ; 
- De développer un partenariat Etat/privé, avec des fora annuels en vue de prévenir l'exploitation économique des enfants et des jeunes ;

- D'instituer un « fonds de compensation communautaire " dans les zones à forte production industrielle en vue de financer les investissements scolaires et l'appui aux groupes vulnérables ;

- D'étudier les options possibles pour une augmentation progressive de la participation à l'enseignement secondaire pour les filles et les garçons ;

- Améliorer le système de pilotage (communication entre acteurs au niveau central mais aussi entre niveau central et niveau local) ;

- Mettre en place des mécanismes au niveau provincial en vue d'une sensibilisation et d'information sur les potentialités économiques et intellectuelles des provinces. 


\section{Conclusion}

La recherche sur les Enfants et Adolescents en Dehors de l'Ecole (EADE) en République Démocratique du Congo (RDC) avait pour objectifs spécifiques de mesurer l'ampleur des EADE en RDC («Combien sont-ils?»), de donner leur localisation géographique («Où sont-ils ? »), de décrire leur profil socio-démographique et économique ("Qui sont-ils"), d'examiner les barrières et goulots d'étranglement à leur scolarisation («Pourquoi sont-ils en dehors de l'école ?») et de proposer des éléments de politique pour accroître leur participation à l'école ("Quels sont l'effectivité et l'impact des politiques et stratégies dans les secteurs de l'éducation et de la protection sociale ?»).

\section{Ampleur et profil des EADE en RDC}

Les résultats extrapolés de l'enquête EADE-RDC 2012 donne une estimation de 7375875 d'enfants et d'adolescents de 5-17 ans en dehors de l'école en 2012, soit en termes relatifs, $28,9 \%$ des 5-17 ans. L'ampleur des EADE est plus forte chez les filles (31,7\%) que chez les garçons $(26,5 \%)$. Si on s'en tient à la tranche d'âge du primaire qui constitue la tranche d'âge de la scolarisation obligatoire, l'effectif des EADE de 6-11 ans est estimé à 3509 252, ce qui représente 47,6\% de l'ensemble des $\operatorname{EADE}(26,8 \%$ des $6-11$ ans).

L'évolution de la proportion des enfants en dehors de l'école révèle que le phénomène est en baisse régulière depuis 2007, passant de 38,5\% en 2007 (EDS 2007), à 32,5\% en 2010 (MICS 2010) et $28,9 \%$ en 2012 (EADE 2012).

En termes absolus (comme en termes relatifs), c'est en milieu rural qu'on retrouve le plus grand nombre des EADE, soit 5694525 d'EADE en milieu rural contre 1681391 en milieu urbain. C'est aussi en milieu rural que l'ampleur du phénomène en termes relatifs est la plus forte $(33,4 \%$ en milieu rural contre $20,0 \%$ en milieu urbain). L'analyse spatiale de l'ampleur du phénomène révèle que c'est dans les provinces à forte production minière et celles où les conflits sont récurrents, que l'ampleur du phénomène est la plus forte (Nord Kivu, Katanga, Kassaï occidental, Province orientale, Sud-Kivu, Kassaï oriental).

Par ailleurs, l'analyse du profil des EADE révèle que ceux-ci sont davantage dans les ménages les plus pauvres $(64,3 \%$ des $6-11$ ans ; $60,4 \%$ des $12-13$ ans et $60,2 \%$ des $14-17$ ans), dans les ménages dont le chef n'a aucun niveau d'instruction $(65,3 \%$ des $6-11$ ans ; $46,5 \%$ des $12-13$ ans et $50,8 \%$ des $14-17$ ans) et qu'ils sont davantage des filles $(50,1 \%$ des $6-11$ ans; $60,4 \%$ des $12-13$ ans et $63,8 \%$ des $14-17$ ans).

Un des apports de l'enquête EADE a été de saisir l'importance du phénomène des enfants et adolescents en situation de rupture familiale (dans des structures d'accueil ou dans la rue). L'étude a ainsi recensé 19414 personnes dans la rue ou dans les structures d'accueil dont 11979 en situation de rupture familiale, la majorité étant des enfants d'âge scolaire (9 410 enfants et adolescents de 5-17 ans). Les enfants et adolescents dans la rue sont surtout concentrés à Kinshasa, au Sud-Kivu, au Nord-Kivu, au Kasaï oriental et dans la Province Orientale. En termes de participation scolaire, la plupart des enfants et adolescents dans la rue sont des déscolarisés et c'est au niveau du primaire qu'ils ont en majorité arrêté leurs études. Par ailleurs, contrairement à ce qu'on aurait pu penser, plus d'un enfant et adolescent de la rue sur trois est non orphelin. En ce qui concerne les enfants et adolescents accueillis dans les structures, le Nord-Kivu vient en tête, suivi du Sud-Kivu et de Kinshasa. Les enfants et adolescents dans les structures sont généralement scolarisés.

Malgré les progrès réalisés dans la scolarisation des enfants et des adolescents en RDC, il reste encore un bon bout de chemin à parcourir en vue la scolarisation primaire universelle. Ceux qui sont exclus du système sont ceux dans les zones les plus reculées, n'ayant pas les ressources financières nécessaires et n'étant pas dans l'environnement familial propice pour augmenter leurs chances de scolarisation. 


\section{Obstacles et goulots d'étranglement}

L'analyse des obstacles montre que les facteurs qui renforcent l'exclusion des enfants de l'école sont multiples et relèvent de la demande scolaire (déterminants socio-culturels et économiques), de l'offre scolaire ainsi que des politiques.

Au niveau de la demande scolaire, les résultats issus des données quantitatives comme des données qualitatives, révèlent la persistance des inégalités de genre dans l'éducation. Si des efforts ont été déployés par l'État et ses partenaires pour l'amélioration de las scolarisation ces dernières années, ayant permis de réduire de façon significative les inégalités d'accès à l'école entre garçons et filles, notamment au primaire, les inégalités de genre (en défaveur des filles) existent toujours au niveau secondaire, surtout en milieu rural. Par ailleurs, la survie des parents est apparue une barrière importante à la participation scolaire des enfants et des adolescents. Le confiage des enfants est apparu aussi comme un des déterminants de l'exclusion scolaire, dans la mesure où il est ressorti des analyses faites que les enfants confiés, particulièrement en milieu urbain, ont un risque plus grand de se retrouver en dehors de l'école que les enfants biologiques du chef de ménage. Le niveau d'éducation des parents est aussi ressorti comme un des déterminants de l'exclusion scolaire des enfants et des adolescents et cela, quel que soit le milieu de résidence ; les enfants vivant dans des ménages dont le chef n'a aucun niveau d'instruction courant un plus grand risque d'être en dehors de l'école. Cette faiblesse du capital éducatif des parents expliquerait aussi en partie les perceptions des parents sur les droits des enfants, perceptions non toujours favorables à la promotion de la protection et des droits des enfants.

Sur le plan économique, le revenu du ménage détermine la fréquentation scolaire des enfants et des adolescents. Que ce soit en milieu urbain ou en milieu rural, le faible revenu du ménage constitue une importante barrière à la scolarisation des enfants tant au niveau primaire que secondaire. Cette barrière est la principale raison invoquée par les ménages pour justifier la non inscription à l'école ou l'abandon scolaire des enfants, elle est aussi confirmée par les propos des acteurs interviewés lors des entretiens qualitatifs.

Au niveau de l'offre scolaire, il ressort que la disponibilité et la qualité des infrastructures scolaires, de l'équipement et du personnel enseignant aux trois niveaux de l'enseignement constituent aussi des déterminants de la participation scolaire des enfants et des adolescents. Bien qu'on note globalement une augmentation de l'offre scolaire ces dernières années à tous les niveaux de l'enseignement, il existe des disparités entre provinces et entre milieux urbain et rural quant à la disponibilité et à la répartition des infrastructures scolaires. Au niveau du pré-primaire, une grande part des écoles maternelles se trouve en milieu urbain. Au niveau du primaire et du secondaire, le nombre d'écoles a considérablement augmenté ces dernières années, mais les écoles primaires et surtout secondaires sont encore inégalement réparties entre les provinces. Dans le même temps, les analyses réalisées montrent que la distance à l'école constitue un des principaux déterminants de l'exclusion scolaire en milieu rural. Par ailleurs, l'offre scolaire est inadaptée aux besoins de certains groupes spécifiques tels que les enfants et adolescents vivant avec un handicap.

Au niveau politique, il ressort clairement que le faible niveau de financement de l'éducation par l'Etat, qui contraint les ménages à consacrer une part importante de leur revenu annuel aux dépenses d'éducation ( $14 \%$ en milieu urbain et $7 \%$ en milieu rural), constitue l'un des déterminants de l'exclusion scolaire. II en est de même de la faiblesse de la capacité institutionnelle et humaine du secteur de l'éducation. Ce problème de capacité institutionnelle et humaine se pose au niveau du fonctionnement des comités de gestion des écoles, au niveau de la gestion des ressources humaines et au niveau de la coordination et de la gestion du système éducatif. En ce qui concerne la gestion des ressources humaines du secteur de l'éducation, plusieurs difficultés sont soulignées dans les entretiens qualitatifs, à savoir l'insuffisance et l'irrégularité des salaires des enseignants, l'absence d'un système de retraite pour les enseignants, le manque de formation de certains enseignants, le non renouvellement du personnel enseignant, etc.

\section{Politiques et stratégies}

En termes de politiques et stratégies pour éliminer les barrières à la participation scolaire des enfants, l'étude EADE-RDC sur la base des résultats de l'enquête nationale, notamment ceux du volet qualitatif, des contributions des acteurs du système éducatif à la suite de la présentation des premiers résultats de la recherche, mais aussi sur la base des expériences dans d'autres contextes, a identifié 
un certain nombre de politiques ou de stratégies, structurées autour du même regroupement que les barrières et goulots d'étranglements.

Au niveau socio-culturel, pour répondre aux problèmes des représentations sociales défavorables aux filles, il est proposé d'intensifier les campagnes de sensibilisation pour la scolarisation des filles, particulièrement au niveau du secondaire, de mettre en place des programmes d'octroi de bourses et envisager comme cela a été expérimenté ailleurs, des dotations incitatives aux ménages. Pour éliminer le phénomène des mariages précoces, il est suggéré de faire appliquer la législation en matière d'âge légal au mariage et pour les grosses précoces, d'intensifier les cours d'éducation en santé sexuelle et reproductive. En ce qui concerne le faible niveau d'instruction et d'alphabétisation des parents et des perceptions sur les droits des enfants, il est suggéré de mettre en place des campagnes de sensibilisation orientées vers les chefs de ménage non instruits/non alphabétisés, de renforcer les programmes d'alphabétisation, de renforcer les compétences socio-économiques des jeunes et des adultes, et d'augmenter la connaissance des acteurs sur les lois relatives à la protection et au droit de l'enfant. Pour ce qui est des effets du mauvais confiage, il est proposé de développer des programmes d'éducation parentale afin de rappeler aux parents leurs obligations envers leurs enfants. Enfin, pour accroître les capacités et les ressources des communautés, il est proposé de mettre en place des approches multisectorielles pour renforcer les capacités institutionnelles et communautaires d'autonomisation en matière de mobilisation de ressources financières pour amener les populations vulnérables à réaliser leurs potentialités économiques dans leur environnement immédiat.

Au niveau des barrières économiques, le rôle important joué par la pauvreté comme barrière à la scolarisation des enfants conduit à réaffirmer l'urgence qu'il y a d'accélérer et de rendre effective la réduction des frais scolaires, comme déjà stipulé dans le Document de Stratégie de développement du sous-secteur de l'enseignement primaire, secondaire et professionnel 2010/2011-2015/2016. En ce qui concerne le travail des enfants, particulièrement le travail dangereux, les propositions vont dans le sens du renforcement des lois sur la protection et les droits des enfants et adolescents et le développement ou le renforcement des programmes d'éducation parentale.

Au niveau de l'offre, il ressort la nécessité d'un accroissement substantiel les infrastructures, du personnel enseignant en nombre et en qualité (particulièrement la proportion des femmes enseignantes pour accroître la rétention des filles à l'école), la nécessité d'une amélioration substantielle de la rémunération des enseignants tout en leur assurant un plan de carrière adéquat, de la nécessité de mettre en place des approches pédagogiques spécifiques pour chaque catégorie d'enfant vivant avec un handicap, et le renforcement des initiatives tendant à rendre possible la scolarisation des enfants de la rue et celles tendant à permettre la scolarisation des enfants et adolescents dans les sites miniers.

Au plan politique et de la gouvernance, les propositions de faites vont dans le sens d'accroître de manière substantielle les ressources de l'Etat allouées à l'éducation, de développer ou renforcer le partenariat entre l'Etat et le monde privé, comme cela existe dans plusieurs pays, à travers des rencontres annuelles permettant non seulement de prévenir de l'exploitation économique des enfants et des jeunes, mais aussi d'instituer et d'alimenter ce qu'on pourrait appeler un " fonds de compensation communautaire " dans les zones à forte production industrielle, qui servirait non seulement à contribuer au financement des investissements dans l'éducation mais aussi à alimenter un fonds pour la protection sociale. II est proposé également, compte tenu de la persistance des inégalités de genre au niveau des âges du secondaire, d'étudier les options possibles pour une augmentation progressive de la participation à l'enseignement secondaire pour les filles et les garçons. L'amélioration du système de pilotage (communication entre acteurs au niveau central mais aussi entre niveau central et niveau local) apparaît aussi comme une exigence pour accompagner toutes ces actions qui seront entreprises pour booster les indicateurs de participation scolaire.

Enfin, de manière plus globale, il faut résolument une démarche originale et volontariste en vue de briser le cycle de la pauvreté et de la dépendance : en cela, aider les communautés à percevoir les diverses potentialités économiques de leur environnement (non seulement les richesses minières, mais aussi les richesses agro-pastorales, les ressources halieutiques, le tourisme, etc.) et leur procurer les bases (en termes de compétences et de crédits) pour les exploiter, permettra finalement aux ménages de pouvoir se prendre davantage en charge et investir durablement dans la scolarisation des enfants. 


\section{Bibliographie}

AINSWORTH, Martha (1992), Economic Aspects of Child Fostering in Côte d'Ivoire, Living Standards Measurement Study, Working Paper $N^{\circ}$ 92, Banque Mondiale, Washington, $43 \mathrm{p}$.

AINSWORTH, Martha, and Deon FILMER (2002). Poverty, AIDS, and Children's Schooling. A targeting Dilemma. The World Bank Policy Research Working Paper (2885):41.

ANH TRUONG, Si, John KNODEL, David LAM et Jed FRIEDMAN (1998), "Family Size and Children's Education in Vietnam », Demography, Vol. 35, №1, p. 57-70.

BAILEY, Sarah, PEREZNIETO, Paola et JONES, Nicolas (2011), Protection sociale adaptée aux besoins des enfants en RDC. Etude diagnostique, Overseas Development Institute, $99 \mathrm{p}$.

Banque Mondiale (2003), Genre et développement économique : vers l'égalité des sexes dans les droits, les ressources et la participation, éditions Saint Martin, 350p.

BANQUE MONDIALE (2005), Le renouveau du système éducatif de la République Démocratique du Congo : Priorités et alternatives, Washington, $184 \mathrm{p}$.

Banque Mondiale (2011), Stratégies de construction scolaire pour l'éducation primaire universelle en Afrique. Faut-il habiliter les communautés à construire leurs écoles ? 302p.

BRISSET, C. (2000), " Le travail des enfants », Problèmes politiques et sociaux, № 839, Paris, 72 p.

BUCHMANN, Claudia, et Emily HANNUM (2001) «Education and stratification in developing countries: A review of theories and research", Annual Review of Sociology 27, p.77-102.

CASE, Anne, Christina PAXON, et Joseph ABLEIDINGER (2004), «Orphans in Africa; Parental death, poverty, and school enrolment», Demography, 4, n³, p. 483-508.

CHERNICHOVSKY, Dov (1985), "Socioeconomic and Demographic Aspects of School Enrollment and Attendance in Rural Botswana », Economic Development and Cultural Change, Vol. 33, $\mathrm{N}^{\circ}$ 2, p. 319-332.

CRAISSATI, Dina (2011), Conceptual and methodological framework mail 1: profiles, UNICEF, 3 p.

DE VREYER, Philippe (1993), "Une analyse économétrique de la demande d'éducation en Côted'Ivoire ", Revue d'économie du développement, Vol. 3, p. 51-79.

DEGRAFF, Deborah S., Richard E. BILSBORROW et Alejandro N. HERRIN (1996), "Children's education in the Philippines: Does high fertility matter? ", Population Research and Policy Review, No 15 , p. 219-247.

DIAGNE Abdoulaye, Ismaël Kafando et Moussa H. Ounteni (2006), Pourquoi les enfants quittent-ils l'école ? Un modèle hiérarchique multinomial des abandons dans l'éducation primaire au Sénégal, Les Cahiers du SISERA, 30p.

DIALLO, Boubacar Bayero, (2006), Les parcours scolaires atypiques du collège de Porédaka en guinée (Afrique de l'ouest): approche comparative, Revista Diálogo Educacional, v. 6, n.17, p. 45-64, jan./abr. 2006.

DIALLO, Y. (2006) Note méthodologique. Du cadre conceptuel aux estimations des formes de travail des enfants : une approche statistique. Version provisoire, $15 \mathrm{p}$.

DURAND, Marie-Hélène, (2006), Les enfants non scolarisés en milieu urbain : une comparaison des déterminants intra familiaux, inter familiaux et des effets de voisinage dans sept capitales ouest africaines, Document de travail, DIAL, 41p.

EDS-RDC (2007), Enquête démographique et de santé de la République démographique du Congo.

FILMER, Deon et Lant H. PRITCHETT (2001), « Estimating Wealth Effects without Expenditure Dataor Tears: an Application to Educational Enrollments in States India », Demography, Vol. 38, № 1, p. 115-132.

FOSTER G., SHAKESPEARE R., CHINEMANA F., JACKSON H., GREGSON S. MARANGE C., and MASHUMBA S. (1995), "Orphan Prevalence and Extended Family Care in Peri-Urban Community in Zimbabwe", AIDS Care, 7, $\mathrm{n}^{\circ} 1,3$.

FOSTER, G., R. SHAKESPEARE, F. CHINEMANA, H. JACKSON, S. GREGSON, C. MARANGE, and S. MASHUMBA. 1995. "Orphan Prevalence and Extended Family Care in a Peri-urban Community in Zimbabwe". Aids Care 7 (1):3-17.

FOX, William (1999), Statistiques sociales, Traduit de l'anglais et adapté par Louis M. Imbeau, Les Presses de l'Université Laval/De Boeck Université, Saint Nicolas, 374 p.

GERTLER, Paul, David I. LEVINE, and Minnie AMES. 2004. "Schooling and Parental Death." The Review of Economics and Statistics 86 (1):211-225.

GILLES, Alain (1994), Éléments de méthodologie et d'analyse statistique pour les sciences sociales, McGraw-Hill, Éditeurs, Montréal, 571 p.

GOMES, Melba (1984), "Family Size and Educational Attainment in Kenya », Population and Development Review, Vol. 10, № 4, p. 647-660. 
Government of Ghana and UNICEF (2012), Global Initiative on Out-of-School Children. Ghana Country Study, $170 \mathrm{p}$.

HAGENAARS, Aldi et Klaas DE VOS (1988), "The Definition and Measurement of Poverty », The Journal of Human Resources, Vol. 23, № 2, p. 211-221.

HAMMOND, Cathy, Dan Linton, Jay Smink and Sam Drew (2007), Drop out risk factors and exemplary programs: A technical report. Clemson, SC: National Dropout Prevention Center.

HILL, Anne M. et Elizabeth M. KING (1993), «Women's Education in Developing Countries: An Overview » in : Elizabeth M. KING et M. Anne HILL (éd.), Women's Education in Developing Countries. Barriers, Benefits, and Policies, Banque Mondiale, The Johns Hopkins University Press, Baltimore, p. 1-50.

HTSPE - EUROPE (2012), Etude sur les déterminants des abandons scolaires au Niger, rapport final, $124 p$.

ISIUGO-ABANIHE Uche C. (1985) "Child forestage in West-Africa», Population and Development Review, vol.11, n¹, p.53-73.

International Labour Organization (ILO) 2009. Report of the Conference: 18th International Conference of Labour Statisticians, Geneva, 24 November-5 December 2008.

KAMUZORA, L. C. (1984), "High Fertility and the Demand for Labor in Peasant Economies : the Case of Bukoba District, Tanzania ", Development and Change, Vol. 15, p. 105-124.

KNODEL, John et Malinee WONGSITH (1991), "Family Size and Children's Education in Thailand : Evidence from a National Sample », Demography, Vol. $28 \mathrm{~N}^{\circ} 1$, p. 119-131.

KOBIANÉ Jean-François (2001), "Revue générale de la littérature sur la demande d'éducation en Afrique ", in : Marc PILON et Yacouba YARO (éditeurs) La demande d'éducation en Afrique. État des connaissances et perspectives de recherche. Collection Réseaux thématiques de recherche de l'U.E.P.A, N 1, pp. 19-47.

KOBIANÉ Jean-François (2004), " Habitat et bien d'équipement comme indicateurs de niveau de vie des ménages : diversité de méthodologies et implications en matière de politique sociale ", Étude de la Population Africaine, Supplément A au Vol. 19, pp. 265-283.

KOBIANÉ Jean-François (2005), "Parental Death and Children's Schooling in Burkina Faso », Comparative Education Review, Vol. 49, No 4, pp. 468-489

KOBIANÉ Jean-François (2006), Ménages et scolarisation des enfants au Burkina Faso : à la recherche des déterminants de la demande scolaire, Collection "Monographies de l'Institut de Démographie de l'UCL », Louvain-la-Neuve, Academia-Bruylant, 306 p.

KOBIANÉ Jean-François (2007) «Ethnies, genre et scolarisation au Burkina Faso : du discours anthropologique aux résultats statistiques ", in : Thérèse $\mathrm{LOCOH}$ (éd.), Genre et sociétés en Afrique. Implications pour le développement. Collection « Les cahiers de I'INED », No. 160, pp. 221-241.

KOBIANÉ, Jean-François (2003), «Pauvreté, structures familiales et stratégies éducatives à Ouagadougou», in Éducation, Famille et Dynamiques Démographiques, Cosio M., Marcoux R., Pilon M., et Quesnel A. (dir.), CICRED, Paris, pp.183-217.

MEPSP-UNICEF-ISSP (2011), Recherche sur les enfants et adolescents en dehors de l'école en démographique et de santé de la République démographique du Congo. Rapport sur l'état des lieux, UNICEF, Kinshasa, $124 \mathrm{p}$.

LANGE, Marie-France (1998), "Introduction », in : Marie-France LANGE (éd.), L'école et les filles en Afrique. Scolarisation sous conditions, Éditions Karthala, Paris, p. 7-26.

LLOYD, Cynthia B. et Ann K. BLANC (1996), "Children's Schooling in sub-Saharan Africa: The Role of Fathers, Mothers, and Others ", Population and development review, Vol. 22, N², p. 265298.

MARCOUX, Richard, Amadou NOUMBISSI, an Tukufu ZUBERI (2005) « Living arrangements of orphans in sahelian countries" (paper presented at the annual meeting of the population association of merica, May 1-3, Minneapolis, 2003).

MEILLASSOUX, Claude (1992), Femmes greniers et capitaux, Éditions L'Harmattan, Paris, 251 p.

METELA S., (2010), "Physionomie démographique de la République Démocratique du Congo de 1957 à 2007 : évolution et facteurs explicatifs ", Actes du colloque sur les questions de population, développement et pauvreté en RDC, pp. 44-55. (A paraître).

MICS-RDC (2010), Enquête à indicateurs multiples de la République démographique du Congo, Kinshasa.

MOUMMI, Ahmed (2010), "Analyse de la pauvreté en République démocratique du Congo », African Development Bank Group, Working Paper № 112, 25 p.

NGONDO P. (2001), « Physionomie démographique de la République Démocratique du Congo et de l'Afrique, " Document Pédagogique $n^{\circ}$ 2, Université de Kinshasa, Faculté des Sciences 
Economiques et de Gestion, Département des Sciences de la Population et du Développement, PUK, Kinshasa, $113 p$.

NYANGARA Florence (2004), Sub-national distribution and situation of orphans : An analysis of the president's emergency plan for Aids Relief Focus Countries (USAID, Bureau of Africa, Washington, DC, 2004), 33.

NYANGARA, Florence. 2004. Sub-National Distribution and Situation of Orphans: An Analisys of the President's Emergency Plan for AIDS Relief Focus Countries: USAID, Bureau of Africa.

POTVIN Pierre, DESLANDES Rollande, BEAULIEU Paula, MARCOTTE Diane, FORTIN Laurier, ROYER Égide et LECLERC Danielle (1999), Risque d'abandon scolaire, style parental et participation parentale au suivi scolaire, REVUE CANADIENNE DE L'ÉDUCATION 24, 4 (1999) : 441-453.

PILON, Marc (2005), "Confiage et scolarisation en Afrique de l'Ouest: Eclairages à partir des sources de données démographiques ", Communication au Congrès de l'UIESP de Tour, $17 \mathrm{p}$.

PILON, Marc et Denis CLEVENOT (1996), "Femmes et scolarisation des enfants », Communication au Séminaire international "Femmes et gestion des ressources", IFORD, Yaoundé, 5-7 février 1996, $24 \mathrm{p}$.

Programme National Multisectoriel de Lutte contre le Sida de la RDC, (2009), Plan stratégique national de lutte contre le Sida (2010-2014), $116 \mathrm{p}$.

RDC (2009), «Loi № 09/001 portant protection de l'enfant du 10 janvier 2009 », Journal Officiel, Numéro spécial, pp 5-6.

RDC (2010), The democratic republic of Congo's country snapshot, Socio-Economic, Macroeconomic Environment and trend in social spending and Characteristics of the social Protection System. Kinshasa, $4 \mathrm{p}$.

RDC/MEPSP (2010), Stratégie pour le développement du sous-secteur de l'EPSP 2010/20112015/2016, Kinshasa, 87 p.

RDC/MEPSP (2012), Plan intérimaire de l'éducation 2012/2014, Kinshasa, 110 p.

ROTH, C. (1996), La séparation des sexes chez les Zara au Burkina Faso, Éditions L'Harmattan, Paris, $254 \mathrm{p}$.

SALAZAR, M. C. (1998), "Child Work and Education in Latin America », in : M. C. Salazar et W. A. Glasinovich (éd.), Child Work and Education. Five case studies from Latin America, UNICEF, Florence, pp. 1-19.

SATHAR, Zeba A. et Cynthia B. LLOYD (1993), "Who Gets Primary Schooling in Pakistan: Inequalities Among and Within Families ", Working Papers № 52, New York, The Population Council Research Division, $43 \mathrm{p}$.

SCHLEMMER, B. (1996), « Présentation générale », in : Bernard SCHLEMMER (éd.), L'enfant exploité. Oppression, mise au travail, prolétarisation, Karthala-ORSTOM, Paris, pp. 7-27.

SEN, Amartya (1992), Inequality Reexamined, Oxford, Clarendon Press.

SHAPIRO, David (1999), "Family Influences on Women's Educational Attainment in Kinshasa », Communication au Séminaire International CICRED "Stratégies éducatives, familles et dynamiques démographiques ", Ouagadougou, Burkina Faso, 15-19 novembre 1999, 20 p.

UCW/UNICEF/IOL/WBG (2011), Child labour and out-of-school children: a statistical profile. Italia, Rome, $16 \mathrm{p}$.

UNDP (2009), World population prospects : The 2008 revision. New York: UNDP.

UNESCO (2000), Forum mondial sur l'éducation. Cadre d'action de Dakar, Paris, 78 p.

UNESCO (2008), Rapport mondial de suivi de l'EPT 2009. Vaincre l'inégalité : l'importance de la gouvernance, Éditions UNESCO, Paris, $490 \mathrm{p}$.

UNESCO (2010), Rapport mondial de suivi de l'EPT 2010. Atteindre les marginalisés, Éditions UNESCO, Paris, $538 \mathrm{p}$.

UNESCO (2012), Rapport mondial de suivi de l'EPT 2012. Jeunes et compétences : l'éducation au travail, Éditions UNESCO, Paris, 524 p.

UNICEF (1997), La situation des enfants dans le monde 1997, New York, 116 p.

UNICEF et UNESCO Institute of statistics (2011), Global initiative on out-of-school children: Conceptual and methodological framework (CMF). New York and Montreal: UNICEF and UIS, March $16 \mathrm{p}$.

URASHA, Mark, J.Ties BOERMA, Japheth Z.L.NG'WSHERMI, Raphael ISINGO, Dick SCHAPINK, and Yusufu KUMOGOLA (1997), "Orphanhood, child fostering and the Aids Epidemic in Rural Tanzanie», Health Transition Review 7, suppl.2, 141-153.

WAKAM, Jean (2002), "La situation des enfants orphelins en matière de scolarisation en Afrique : le cas du Cameroun», in Jeunesses, vieillesses, démographies et sociétés, Chaire Quetelet 
2001/Reseau démographie de l'AUF, ed. Francis Gendreau, Dominique Tabutin, and Maud Poupard (Louvain-la-Neuve : Academia-Bruylant/L'Harmattan, 2002), pp.177-195.

WAKAM, Jean (2003), "Structures démographiques des ménages et scolarisation des enfants au Cameroun", in Éducation, Famille et Dynamiques Démographiques, Cosio M., Marcoux R., Pilon M., et Quesnal A. (dir.), CICRED, Paris, pp.183-217.

WONNACOTT, Thomas H. et Ronald J. WONNACOTT (1991), Statistique. Économie - gestion sciences - médecine (avec exercices d'application). Quatrième édition, Economica, Paris, 919 p.

World Bank (2001), World Development Report 2000/2001. Attacking Poverty, Oxford, Oxford University Press.

World Bank (2006), World Development Report 2007. Development and the Next Generation, Washington, $270 \mathrm{p}$.

ZOUNGRANA, Cécile, Joël Marie TOKINDANG, Richard MARCOUX et Mamadou KONATÉ (1998), "La trajectoire scolaire des filles à Bamako. Un parcours semé d'embûches », in : Marie-France LANGE (éd.), L'école et les filles en Afrique. Scolarisation sous conditions, Éditions Karthala, Paris, p. 167-696. 


\section{Annexe 1 : Les erreurs de sondage}

Les estimations des erreurs de sondage s'inspirent de la méthodologie utilisée pour les EDS (http://measuredhs.com/). Les estimations obtenues à partir d'une enquête par sondage sont sujettes à deux types d'erreurs : les erreurs de mesure et les erreurs de sondage. Les erreurs de mesure sont celles associées à la mise en œuvre de la collecte et de l'exploitation des données telles que l'omission des enfants des ménages sélectionnés, la mauvaise interprétation des questions de la part des enquêteurs ou des enquêtés, ou les erreurs de saisie des données. Malgré les précautions techniques prises pour minimiser ce type d'erreur pendant la mise en œuvre de l'EADE-RDC 2012, il est difficile d'éviter et d'évaluer toutes les erreurs de mesure.

Par contre, les erreurs de sondage peuvent être évaluées statistiquement. L'échantillon sélectionné pour l'EADE-RDC 2012 n'est qu'un parmi un grand nombre d'échantillons de même taille qui peuvent être sélectionnés dans la même population avec le même plan de sondage. Chacun de ces échantillons peut produire des résultats peu différents de ceux obtenus avec l'échantillon actuellement choisi. L'erreur de sondage est une mesure de cette variabilité entre tous les échantillons possibles. Bien que cette variabilité ne puisse pas être mesurée exactement, elle peut être estimée cependant, à partir des données collectées.

L'erreur-type $(E T)$ est un indice particulièrement utile pour mesurer l'erreur de sondage d'un paramètre (moyenne, proportion ou taux), elle est la racine carrée de la variance du paramètre. L'erreur-type peut être utilisée pour calculer des intervalles de confiance dans lesquels nous estimons que se trouve la vraie valeur du paramètre avec un certain niveau de confiance. Par exemple, la vraie valeur d'un paramètre se trouve dans les limites de sa valeur estimée plus ou moins deux fois de son erreurtype, avec un niveau de confiance de $95 \%$.

L'échantillon de l'EADE-RDC 2012 est un échantillon stratifié tiré à deux ou trois degrés. Le plan de sondage ne répond pas à un sondage aléatoire simple. On ne peut donc pas utiliser les formules simples pour calculer les erreurs de sondage.

La méthode de linéarisation (Taylor) a été utilisée pour l'estimation des proportions et la méthode de Jackknife est utilisée pour des estimations plus complexes (exemple dans l'EDS de l'indice synthétique de fécondité et des quotients de mortalité).

La méthode de linéarisation traite chaque proportion ou moyenne comme étant une estimation de ratio, $r=y / x$, avec $y$ la valeur du paramètre pour l'échantillon total, et $x$ le nombre total de cas dans l'ensemble (ou sous-ensemble) de l'échantillon. La variance de $r$ est estimée par :

$$
E T^{2}(r)=\operatorname{var}(r)=\frac{1-f}{x^{2}} \sum_{h=1}^{H}\left[\frac{m_{h}}{m_{h-1}}\left(\sum_{i=1}^{m_{h}} z_{h i}^{2}-\frac{z_{h}^{2}}{m_{h}}\right)\right]
$$

dans laquelle

$$
z_{h i}=y_{h i}-r x_{h i}, \text { et } z_{h}=y_{h}-r x_{h}
$$

où $\quad h$ représente la strate qui va de 1 à $H$,

$m_{h}$ est le nombre total de grappes tirées dans la strate $h$,

$y_{h i}$ est la somme des valeurs pondérées du paramètre $y$ dans la grappe $i$ de la strate $h$,

$x_{h i}$ est la somme des nombres pondérés de cas dans la grappe $i$ de la strate $h$, et

$f \quad$ est le taux global de sondage qui est négligeable.

La méthode de Jackknife dérive les estimations des taux complexes à partir de chacun des souséchantillons de l'échantillon principal, et calcule les variances de ces estimations avec des formules simples. Chaque sous-échantillon exclut une grappe dans les calculs des estimations. Ainsi, des souséchantillons pseudo-indépendants ont été créés. Dans l'EADE-RDC 2012, il y a 454 grappes nonvides. Par conséquent, 454 sous-échantillons ont été créés. La variance d'une proportion rest calculée de la façon suivante : 


$$
E T^{2}(r)=\operatorname{var}(r)=\frac{1}{k(k-1)} \sum_{i=1}^{k}\left(r_{i}-r\right)^{2}
$$

dans laquelle

$$
r_{i}=k r-(k-1) r_{(i)}
$$

où $\quad$ rest l'estimation calculée à partir de l'échantillon principal de 454 grappes,

$r_{(i)}$ est l'estimation calculée à partir de l'échantillon réduit de 453 grappes

$$
\text { (ìme } \text { grappe exclue), }
$$

$k$ est le nombre total de grappes.

Les erreurs de sondage pour l'EADE-RDC 2012 ont été calculées pour certaines des variables les plus pertinentes à partir de macros élaborés sous Excel. Les résultats sont présentés dans cette annexe pour l'ensemble de la RDC et les onze provinces. Les tableaux suivant présentent la valeur de la statistique $(p)$, l'erreur-type $(E T)$, le nombre de cas pondérés $(N)$, l'erreur relative $(E T / M)$, et l'intervalle de confiance à $95 \%(\mathrm{M} \pm 2 \mathrm{ET})$ pour chaque variable.

L'intervalle de confiance est interprété de la manière suivante : pour la variable Enfants de 5-17 ans, l'EADE-RDC 2012 a donné une proportion d'EADE de 0,290 (29,0\%) pour l'ensemble des enfants de 5-17 ans, auquel correspond une erreur-type de 0,009 enfants. Dans $95 \%$ des échantillons de taille et caractéristique identiques, la valeur réelle de la proportion d'EADE parmi les enfants de 5-17 ans se trouve entre $0,290-2 \times 0,009$ et $0,290+2 \times 0,009$, soit 0,272 et 0,307 .

Les erreurs de sondage ont été analysées pour l'échantillon national d'enfants et pour les proportions. Les erreurs relatives (ET/M) des proportions se situent entre $1,6 \%$ et $11,5 \%$ avec une moyenne de $4,6 \%$. Les erreurs relatives les plus élevées sont généralement celles des estimations de très faible valeur (par exemple, parmi les enfants des ménages de 200 USD et plus de revenu mensuel qui sont EADE). En général, les erreurs relatives de la plupart des estimations pour l'ensemble du pays sont faibles, sauf dans le cas de très faibles proportions. L'erreur relative de la proportion d'EADE de 5-17 ans est assez faible 3,0\%. Cependant, pour la proportion d'EADE de 12-13 ans et du milieu urbain, l'erreur relative est plus élevée $7,3 \%$ et $7,5 \%$.

II existe aussi des différences entre les erreurs relatives au niveau des sous-échantillons. Par exemple, pour la proportion d'EADE de 5-17 ans, l'erreur relative est respectivement de 11,6\%, 9,3\% et 3,0\% pour le Kasaï Occidental, Kinshasa et l'ensemble de la RDC. 
Tableau des erreurs de sondage, Echantillon national

\begin{tabular}{|c|c|c|c|c|c|c|}
\hline \multirow{2}{*}{$\frac{\text { RDC }}{\text { Variables }}$} & \multirow{2}{*}{$\begin{array}{c}\text { Valeur } \\
\text { p }\end{array}$} & \multirow{2}{*}{$\begin{array}{l}\text { Erreur } \\
\text { type } \\
\text { (ET) }\end{array}$} & \multirow{2}{*}{$\begin{array}{l}\text { Effectif }(\mathrm{N}) \\
\text { pondéré }\end{array}$} & \multirow{2}{*}{$\begin{array}{c}\text { Erreur } \\
\text { relative } \\
(\mathrm{ET} / \mathrm{p})\end{array}$} & \multicolumn{2}{|c|}{ Intervalle de confiance (IC) } \\
\hline & & & & & $\mathrm{p}-2 \mathrm{ET}$ & $p+2 E T$ \\
\hline Enfants de 5-17 ans & 0,290 & 0,009 & 25455282 & 0,030 & 0,272 & 0,307 \\
\hline Enfants de 5 ans & 0,788 & 0,013 & 2720087 & 0,016 & 0,762 & 0,814 \\
\hline Enfants de 6-11 ans & 0,268 & 0,011 & 13117605 & 0,040 & 0,246 & 0,289 \\
\hline Enfants de $12-13$ ans & 0,131 & 0,010 & 3917946 & 0,073 & 0,112 & 0,150 \\
\hline Enfants de $14-17$ ans & 0,212 & 0,011 & 5699644 & 0,051 & 0,190 & 0,234 \\
\hline Garçons & 0,265 & 0,009 & 13158587 & 0,033 & 0,247 & 0,282 \\
\hline Filles & 0,317 & 0,012 & 12296731 & 0,037 & 0,293 & 0,340 \\
\hline Enfants des ménages avec moins de 50 USD & 0,366 & 0,011 & 12457229 & 0,031 & 0,344 & 0,389 \\
\hline Enfants des ménages avec 200 USD et plus & 0,112 & 0,013 & 2584518 & 0,115 & 0,086 & 0,138 \\
\hline CM sans instruction & 0,329 & 0,010 & 14223032 & 0,029 & 0,309 & 0,348 \\
\hline CM de niveau secondaire et plus & 0,187 & 0,010 & 8391118 & 0,051 & 0,168 & 0,207 \\
\hline Enfants du chef de ménage & 0,282 & 0,009 & 20287603 & 0,033 & 0,264 & 0,300 \\
\hline Milieu urbain & 0,200 & 0,015 & 8399085 & 0,075 & 0,169 & 0,231 \\
\hline Milieu rural & 0,334 & 0,011 & 17056197 & 0,032 & 0,313 & 0,355 \\
\hline
\end{tabular}

Tableau des erreurs de sondage, Echantillon province de Kinshasa

\begin{tabular}{|c|c|c|c|c|c|c|}
\hline \multirow{2}{*}{$\begin{array}{l}\text { Kinshasa } \\
\text { Variables }\end{array}$} & \multirow{2}{*}{$\begin{array}{c}\text { Valeur } \\
p\end{array}$} & \multirow{2}{*}{$\begin{array}{l}\text { Erreur } \\
\text { type } \\
\text { (ET) }\end{array}$} & \multirow{2}{*}{$\begin{array}{l}\text { Effectif }(\mathrm{N}) \\
\text { pondéré }\end{array}$} & \multirow{2}{*}{$\begin{array}{l}\text { Erreur } \\
\text { relative } \\
(\mathrm{ET} / \mathrm{p})\end{array}$} & \multicolumn{2}{|c|}{ Intervalle de confiance (IC) } \\
\hline & & & & & $\mathrm{p}-2 \mathrm{ET}$ & $\mathrm{p}+2 \mathrm{ET}$ \\
\hline Enfants de 5-17 ans & 0,157 & 0,015 & 3270503 & 0,093 & 0,127 & 0,187 \\
\hline Enfants de 5 ans & 0,529 & 0,048 & 251280 & 0,091 & 0,431 & 0,628 \\
\hline Enfants de $6-11$ ans & 0,131 & 0,017 & 1626913 & 0,129 & 0,097 & 0,166 \\
\hline Enfants de $12-13$ ans & 0,071 & 0,018 & 479217 & 0,251 & 0,035 & 0,108 \\
\hline Enfants de $14-17$ ans & 0,147 & 0,016 & 913093 & 0,111 & 0,113 & 0,180 \\
\hline Garçons & 0,159 & 0,019 & 1635569 & 0,123 & 0,119 & 0,199 \\
\hline Filles & 0,156 & 0,014 & 1634930 & 0,087 & 0,129 & 0,183 \\
\hline Enfants des ménages avec moins de 50 USD & 0,431 & 0,063 & 100415 & 0,145 & 0,294 & 0,569 \\
\hline Enfants des ménages avec 200 USD et plus & 0,095 & 0,012 & 1571268 & 0,128 & 0,070 & 0,120 \\
\hline CM sans instruction & 0,195 & 0,019 & 1649696 & 0,096 & 0,156 & 0,233 \\
\hline $\mathrm{CM}$ de niveau secondaire et plus & 0,118 & 0,015 & 1600960 & 0,124 & 0,088 & 0,147 \\
\hline Enfants du chef de ménage & 0,130 & 0,015 & 2340953 & 0,113 & 0,100 & 0,159 \\
\hline
\end{tabular}

Tableau des erreurs de sondage, Echantillon province de Bas Congo

\begin{tabular}{|c|c|c|c|c|c|c|}
\hline \multirow{2}{*}{$\begin{array}{l}\text { Bas Congo } \\
\text { Variables }\end{array}$} & \multirow{2}{*}{$\begin{array}{c}\text { Valeur } \\
p\end{array}$} & \multirow{2}{*}{$\begin{array}{l}\text { Erreur } \\
\text { type } \\
(\mathrm{ET}) \\
\end{array}$} & \multirow{2}{*}{$\begin{array}{l}\text { Effectif }(\mathrm{N}) \\
\text { pondéré }\end{array}$} & \multirow{2}{*}{$\begin{array}{l}\text { Erreur } \\
\text { relative } \\
(\mathrm{ET} / \mathrm{p}) \\
\end{array}$} & \multicolumn{2}{|c|}{ Intervalle de confiance (IC) } \\
\hline & & & & & $p-2 E T$ & $p+2 E T$ \\
\hline Enfants de 5-17 ans & 0,271 & 0,017 & 1405175 & 0,062 & 0,237 & 0,305 \\
\hline Enfants de 5 ans & 0,869 & 0,024 & 156527 & 0,028 & 0,820 & 0,918 \\
\hline Enfants de 6-11 ans & 0,224 & 0,020 & 701716 & 0,090 & 0,182 & 0,265 \\
\hline Enfants de $12-13$ ans & 0,121 & 0,024 & 237749 & 0,198 & 0,072 & 0,171 \\
\hline Enfants de $14-17$ ans & 0,191 & 0,023 & 309183 & 0,120 & 0,144 & 0,238 \\
\hline Garçons & 0,235 & 0,015 & 763894 & 0,064 & 0,204 & 0,266 \\
\hline Filles & 0,314 & 0,028 & 641276 & 0,089 & 0,256 & 0,371 \\
\hline Enfants des ménages avec moins de 50 USD & 0,315 & 0,021 & 573933 & 0,067 & 0,270 & 0,359 \\
\hline Enfants des ménages avec 200 USD et plus & 0,085 & 0,029 & 86465 & 0,346 & 0,020 & 0,150 \\
\hline CM sans instruction & 0,330 & 0,019 & 783932 & 0,058 & 0,291 & 0,369 \\
\hline $\mathrm{CM}$ de niveau secondaire et plus & 0,153 & 0,016 & 452881 & 0,105 & 0,121 & 0,186 \\
\hline Enfants du chef de ménage & 0,269 & 0,019 & 1069624 & 0,069 & 0,231 & 0,307 \\
\hline
\end{tabular}


Tableau des erreurs de sondage, Echantillon province de Bandundu

\begin{tabular}{|c|c|c|c|c|c|c|}
\hline \multirow{2}{*}{$\begin{array}{l}\text { Bandundu } \\
\text { Variables }\end{array}$} & \multirow{2}{*}{$\begin{array}{c}\text { Valeur } \\
\mathrm{p}\end{array}$} & \multirow{2}{*}{$\begin{array}{l}\text { Erreur } \\
\text { type } \\
\text { (ET) }\end{array}$} & \multirow{2}{*}{$\begin{array}{l}\text { Effectif }(\mathrm{N}) \\
\text { pondéré }\end{array}$} & \multirow{2}{*}{$\begin{array}{l}\text { Erreur } \\
\text { relative } \\
(\mathrm{ET} / \mathrm{p})\end{array}$} & \multicolumn{2}{|c|}{ Intervalle de confiance (IC) } \\
\hline & & & & & $\mathrm{p}-2 \mathrm{ET}$ & $\mathrm{p}+2 \mathrm{ET}$ \\
\hline Enfants de 5-17 ans & 0,205 & 0,017 & 2811998 & 0,083 & 0,170 & 0,240 \\
\hline Enfants de 5 ans & 0,754 & 0,044 & 309605 & 0,058 & 0,665 & 0,844 \\
\hline Enfants de 6-11 ans & 0,190 & 0,022 & 1445513 & 0,117 & 0,144 & 0,236 \\
\hline Enfants de $12-13$ ans & 0,040 & 0,013 & 456926 & 0,311 & 0,015 & 0,066 \\
\hline Enfants de $14-17$ ans & 0,083 & 0,015 & 599954 & 0,185 & 0,052 & 0,115 \\
\hline Garçons & 0,198 & 0,016 & 1443041 & 0,083 & 0,164 & 0,231 \\
\hline Filles & 0,213 & 0,025 & 1368955 & 0,116 & 0,162 & 0,264 \\
\hline Enfants des ménages avec moins de 50 USD & 0,254 & 0,018 & 1767821 & 0,070 & 0,217 & 0,291 \\
\hline Enfants des ménages avec 200 USD et plus & 0,012 & 0,012 & 24134 & 0,949 & $-0,016$ & 0,041 \\
\hline CM sans instruction & 0,254 & 0,021 & 1498875 & 0,084 & 0,210 & 0,298 \\
\hline CM de niveau secondaire et plus & 0,135 & 0,019 & 1069688 & 0,141 & 0,096 & 0,174 \\
\hline Enfants du chef de ménage & 0,204 & 0,018 & 2275022 & 0,087 & 0,168 & 0,240 \\
\hline
\end{tabular}

Tableau des erreurs de sondage, Echantillon province de l'Equateur

\begin{tabular}{|c|c|c|c|c|c|c|}
\hline \multirow{2}{*}{$\begin{array}{l}\text { Equateur } \\
\text { Variables }\end{array}$} & \multirow{2}{*}{$\begin{array}{c}\text { Valeur } \\
\mathrm{p}\end{array}$} & \multirow{2}{*}{$\begin{array}{l}\text { Erreur } \\
\text { type } \\
(\mathrm{ET})\end{array}$} & \multirow{2}{*}{$\begin{array}{l}\text { Effectif }(\mathrm{N}) \\
\text { pondéré }\end{array}$} & \multirow{2}{*}{$\begin{array}{c}\text { Erreur } \\
\text { relative } \\
(\mathrm{ET} / \mathrm{p})\end{array}$} & \multicolumn{2}{|c|}{ Intervalle de confiance (IC) } \\
\hline & & & & & $\mathrm{p}-2 \mathrm{ET}$ & $p+2 E T$ \\
\hline Enfants de 5-17 ans & 0,279 & 0,024 & 2601109 & 0,085 & 0,230 & 0,328 \\
\hline Enfants de 5 ans & 0,812 & 0,029 & 309567 & 0,036 & 0,753 & 0,872 \\
\hline Enfants de 6-11 ans & 0,255 & 0,026 & 1374165 & 0,101 & 0,202 & 0,308 \\
\hline Enfants de $12-13$ ans & 0,124 & 0,045 & 393595 & 0,366 & 0,030 & 0,218 \\
\hline Enfants de $14-17$ ans & 0,145 & 0,025 & 523782 & 0,174 & 0,093 & 0,198 \\
\hline Garçons & 0,253 & 0,022 & 1344810 & 0,089 & 0,207 & 0,299 \\
\hline Filles & 0,308 & 0,029 & 1256288 & 0,093 & 0,249 & 0,366 \\
\hline Enfants des ménages avec moins de 50 USD & 0,317 & 0,029 & 1860334 & 0,092 & 0,256 & 0,377 \\
\hline Enfants des ménages avec 200 USD et plus & 0,198 & 0,087 & 29572 & 0,437 & $-0,016$ & 0,413 \\
\hline $\mathrm{CM}$ sans instruction & 0,323 & 0,024 & 1511662 & 0,076 & 0,272 & 0,373 \\
\hline $\mathrm{CM}$ de niveau secondaire et plus & 0,161 & 0,022 & 831084 & 0,136 & 0,116 & 0,207 \\
\hline Enfants du chef de ménage & 0,270 & 0,025 & 2240600 & 0,092 & 0,219 & 0,321 \\
\hline
\end{tabular}

Tableau des erreurs de sondage, Echantillon Province Orientale

\begin{tabular}{|c|c|c|c|c|c|c|}
\hline \multirow{2}{*}{$\begin{array}{c}\text { Province Orientale } \\
\text { Variables }\end{array}$} & \multirow{2}{*}{$\begin{array}{c}\text { Valeur } \\
p\end{array}$} & \multirow{2}{*}{$\begin{array}{l}\text { Erreur } \\
\text { type } \\
\text { (ET) }\end{array}$} & \multirow{2}{*}{$\begin{array}{l}\text { Effectif }(\mathrm{N}) \\
\text { pondéré }\end{array}$} & \multirow{2}{*}{$\begin{array}{l}\text { Erreur } \\
\text { relative } \\
(\mathrm{ET} / \mathrm{p})\end{array}$} & \multicolumn{2}{|c|}{ Intervalle de confiance (IC) } \\
\hline & & & & & $p-2 E T$ & $p+2 E T$ \\
\hline Enfants de 5-17 ans & 0,322 & 0,028 & 3223234 & 0,087 & 0,264 & 0,380 \\
\hline Enfants de 5 ans & 0,806 & 0,034 & 361249 & 0,043 & 0,735 & 0,877 \\
\hline Enfants de 6-11 ans & 0,287 & 0,035 & 1641303 & 0,123 & 0,215 & 0,359 \\
\hline Enfants de $12-13$ ans & 0,166 & 0,036 & 507014 & 0,217 & 0,092 & 0,241 \\
\hline Enfants de $14-17$ ans & 0,267 & 0,040 & 713668 & 0,149 & 0,185 & 0,350 \\
\hline Garçons & 0,305 & 0,031 & 1725829 & 0,102 & 0,241 & 0,369 \\
\hline Filles & 0,342 & 0,029 & 1497395 & 0,085 & 0,282 & 0,401 \\
\hline Enfants des ménages avec moins de 50 USD & 0,373 & 0,036 & 1498196 & 0,097 & 0,297 & 0,449 \\
\hline Enfants des ménages avec 200 USD et plus & 0,222 & 0,087 & 234993 & 0,394 & 0,030 & 0,414 \\
\hline CM sans instruction & 0,337 & 0,033 & 1716427 & 0,099 & 0,266 & 0,407 \\
\hline CM de niveau secondaire et plus & 0,244 & 0,026 & 893651 & 0,107 & 0,191 & 0,298 \\
\hline Enfants du chef de ménage & 0,327 & 0,032 & 2367120 & 0,097 & 0,262 & 0,392 \\
\hline
\end{tabular}


Tableau des erreurs de sondage, Echantillon province du Nord Kivu

\begin{tabular}{|c|c|c|c|c|c|c|}
\hline \multirow{2}{*}{$\begin{array}{l}\text { Nord Kivu } \\
\text { Variables }\end{array}$} & \multirow{2}{*}{$\begin{array}{c}\text { Valeur } \\
p\end{array}$} & \multirow{2}{*}{$\begin{array}{l}\text { Erreur } \\
\text { type } \\
\text { (ET) }\end{array}$} & \multirow{2}{*}{$\begin{array}{l}\text { Effectif }(\mathrm{N}) \\
\text { pondéré }\end{array}$} & \multirow{2}{*}{$\begin{array}{l}\text { Erreur } \\
\text { relative } \\
(\mathrm{ET} / \mathrm{p})\end{array}$} & \multicolumn{2}{|c|}{ Intervalle de confiance (IC) } \\
\hline & & & & & $p-2 E T$ & $p+2 E T$ \\
\hline Enfants de 5-17 ans & 0,440 & 0,023 & 2264871 & 0,052 & 0,393 & 0,487 \\
\hline Enfants de 5 ans & 0,959 & 0,010 & 281823 & 0,011 & 0,939 & 0,980 \\
\hline Enfants de 6-11 ans & 0,403 & 0,032 & 1138700 & 0,079 & 0,339 & 0,468 \\
\hline Enfants de $12-13$ ans & 0,261 & 0,029 & 330591 & 0,112 & 0,201 & 0,321 \\
\hline Enfants de $14-17$ ans & 0,352 & 0,034 & 513757 & 0,096 & 0,283 & 0,421 \\
\hline Garçons & 0,420 & 0,026 & 1154308 & 0,063 & 0,366 & 0,474 \\
\hline Filles & 0,461 & 0,024 & 1110563 & 0,051 & 0,413 & 0,510 \\
\hline Enfants des ménages avec moins de 50 USD & 0,519 & 0,025 & 1412368 & 0,048 & 0,468 & 0,571 \\
\hline Enfants des ménages avec 200 USD et plus & 0,268 & 0,086 & 81563 & 0,323 & 0,061 & 0,475 \\
\hline $\mathrm{CM}$ sans instruction & 0,482 & 0,027 & 1311892 & 0,057 & 0,426 & 0,537 \\
\hline $\mathrm{CM}$ de niveau secondaire et plus & 0,273 & 0,023 & 594591 & 0,085 & 0,225 & 0,321 \\
\hline Enfants du chef de ménage & 0,426 & 0,025 & 1865626 & 0,059 & 0,375 & 0,478 \\
\hline
\end{tabular}

Tableau des erreurs de sondage, Echantillon province du Maniema

\begin{tabular}{|c|c|c|c|c|c|c|}
\hline \multirow{2}{*}{$\begin{array}{l}\text { Maniema } \\
\text { Variables }\end{array}$} & \multirow{2}{*}{$\begin{array}{c}\text { Valeur } \\
\mathrm{p}\end{array}$} & \multirow{2}{*}{$\begin{array}{l}\text { Erreur } \\
\text { type } \\
(\mathrm{ET})\end{array}$} & \multirow{2}{*}{$\begin{array}{l}\text { Effectif (N) } \\
\text { pondéré }\end{array}$} & \multirow{2}{*}{$\begin{array}{c}\text { Erreur } \\
\text { relative } \\
(\mathrm{ET} / \mathrm{p})\end{array}$} & \multicolumn{2}{|c|}{ Intervalle de confiance (IC) } \\
\hline & & & & & $\mathrm{p}-2 \mathrm{ET}$ & $\mathrm{p}+2 \mathrm{ET}$ \\
\hline Enfants de 5-17 ans & 0,260 & 0,018 & 720539 & 0,068 & 0,223 & 0,296 \\
\hline Enfants de 5 ans & 0,837 & 0,027 & 92313 & 0,033 & 0,781 & 0,893 \\
\hline Enfants de 6-11 ans & 0,217 & 0,020 & 364228 & 0,091 & 0,177 & 0,258 \\
\hline Enfants de $12-13$ ans & 0,072 & 0,016 & 110601 & 0,224 & 0,039 & 0,105 \\
\hline Enfants de $14-17$ ans & 0,148 & 0,029 & 153397 & 0,195 & 0,088 & 0,208 \\
\hline Garçons & 0,250 & 0,019 & 381484 & 0,078 & 0,210 & 0,289 \\
\hline Filles & 0,271 & 0,023 & 339050 & 0,084 & 0,224 & 0,318 \\
\hline Enfants des ménages avec moins de 50 USD & 0,284 & 0,021 & 493043 & 0,073 & 0,242 & 0,326 \\
\hline Enfants des ménages avec 200 USD et plus & 0,197 & na & 4415 & na & na & na \\
\hline $\mathrm{CM}$ sans instruction & 0,298 & 0,024 & 429421 & 0,079 & 0,250 & 0,346 \\
\hline $\mathrm{CM}$ de niveau secondaire et plus & 0,172 & 0,019 & 224422 & 0,111 & 0,133 & 0,210 \\
\hline Enfants du chef de ménage & 0,263 & 0,018 & 585649 & 0,067 & 0,227 & 0,299 \\
\hline
\end{tabular}

Tableau des erreurs de sondage, Echantillon province du Sud Kivu

\begin{tabular}{|c|c|c|c|c|c|c|}
\hline \multirow{2}{*}{$\begin{array}{l}\text { Sud Kivu } \\
\text { Variables }\end{array}$} & \multirow{2}{*}{$\begin{array}{c}\text { Valeur } \\
\mathrm{p}\end{array}$} & \multirow{2}{*}{$\begin{array}{l}\text { Erreur } \\
\text { type } \\
(\mathrm{ET})\end{array}$} & \multirow{2}{*}{$\begin{array}{l}\text { Effectif }(\mathrm{N}) \\
\text { pondéré }\end{array}$} & \multirow{2}{*}{$\begin{array}{c}\text { Erreur } \\
\text { relative } \\
(\mathrm{ET} / \mathrm{p}) \\
\end{array}$} & \multicolumn{2}{|c|}{ Intervalle de confiance (IC) } \\
\hline & & & & & $\mathrm{p}-2 \mathrm{ET}$ & $\mathrm{p}+2 \mathrm{ET}$ \\
\hline Enfants de 5-17 ans & 0,303 & 0,022 & 1686122 & 0,074 & 0,257 & 0,349 \\
\hline Enfants de 5 ans & 0,760 & 0,062 & 189812 & 0,082 & 0,627 & 0,893 \\
\hline Enfants de 6-11 ans & 0,274 & 0,025 & 860094 & 0,092 & 0,222 & 0,326 \\
\hline Enfants de $12-13$ ans & 0,127 & 0,028 & 281700 & 0,219 & 0,069 & 0,185 \\
\hline Enfants de $14-17$ ans & 0,269 & 0,030 & 354516 & 0,111 & 0,208 & 0,329 \\
\hline Garçons & 0,272 & 0,021 & 859196 & 0,079 & 0,229 & 0,316 \\
\hline Filles & 0,335 & 0,030 & 826948 & 0,089 & 0,273 & 0,396 \\
\hline Enfants des ménages avec moins de 50 USD & 0,406 & 0,030 & 683370 & 0,074 & 0,343 & 0,470 \\
\hline Enfants des ménages avec 200 USD et plus & 0,107 & 0,031 & 88193 & 0,290 & 0,039 & 0,175 \\
\hline CM sans instruction & 0,362 & 0,032 & 1024014 & 0,087 & 0,297 & 0,428 \\
\hline $\mathrm{CM}$ de niveau secondaire et plus & 0,188 & 0,037 & 460694 & 0,196 & 0,106 & 0,269 \\
\hline Enfants du chef de ménage & 0,307 & 0,024 & 1441929 & 0,079 & 0,257 & 0,356 \\
\hline
\end{tabular}


Tableau des erreurs de sondage, Echantillon province du Katanga

\begin{tabular}{|c|c|c|c|c|c|c|}
\hline \multirow{2}{*}{$\begin{array}{l}\text { Katanga } \\
\text { Variables }\end{array}$} & \multirow{2}{*}{$\begin{array}{c}\text { Valeur } \\
p\end{array}$} & \multirow{2}{*}{$\begin{array}{l}\text { Erreur } \\
\text { type } \\
(\mathrm{ET}) \\
\end{array}$} & \multirow{2}{*}{$\begin{array}{l}\text { Effectif }(\mathrm{N}) \\
\text { pondéré }\end{array}$} & \multirow{2}{*}{$\begin{array}{l}\text { Erreur } \\
\text { relative } \\
(\mathrm{ET} / \mathrm{p})\end{array}$} & \multicolumn{2}{|c|}{ Intervalle de confiance (IC) } \\
\hline & & & & & $\mathrm{p}-2 \mathrm{ET}$ & $\mathrm{p}+2 \mathrm{ET}$ \\
\hline Enfants de 5-17 ans & 0,348 & 0,027 & 3833693 & 0,078 & 0,293 & 0,403 \\
\hline Enfants de 5 ans & 0,765 & 0,033 & 415280 & 0,044 & 0,697 & 0,833 \\
\hline Enfants de 6-11 ans & 0,332 & 0,029 & 2036685 & 0,088 & 0,273 & 0,391 \\
\hline Enfants de $12-13$ ans & 0,184 & 0,024 & 574859 & 0,128 & 0,137 & 0,232 \\
\hline Enfants de $14-17$ ans & 0,290 & 0,036 & 806869 & 0,124 & 0,217 & 0,364 \\
\hline Garçons & 0,323 & 0,027 & 2040144 & 0,083 & 0,268 & 0,378 \\
\hline Filles & 0,377 & 0,031 & 1793586 & 0,081 & 0,315 & 0,439 \\
\hline Enfants des ménages avec moins de 50 USD & 0,451 & 0,034 & 1837732 & 0,075 & 0,382 & 0,519 \\
\hline Enfants des ménages avec 200 USD et plus & 0,082 & 0,018 & 401748 & 0,219 & 0,043 & 0,121 \\
\hline CM sans instruction & 0,376 & 0,029 & 2228142 & 0,077 & 0,317 & 0,435 \\
\hline CM de niveau secondaire et plus & 0,208 & 0,024 & 1056731 & 0,114 & 0,160 & 0,256 \\
\hline Enfants du chef de ménage & 0,332 & 0,028 & 3193925 & 0,083 & 0,276 & 0,389 \\
\hline
\end{tabular}

Tableau des erreurs de sondage, Echantillon province du Kasaï Oriental

\begin{tabular}{|c|c|c|c|c|c|c|}
\hline \multirow{2}{*}{$\begin{array}{c}\text { Kasaï Oriental } \\
\text { Variables }\end{array}$} & \multirow{2}{*}{$\begin{array}{l}\text { Valeur } \\
p\end{array}$} & \multirow{2}{*}{$\begin{array}{l}\text { Erreur } \\
\text { type } \\
(\mathrm{ET})\end{array}$} & \multirow{2}{*}{$\begin{array}{c}\text { Effectif }(\mathrm{N}) \\
\text { pondéré }\end{array}$} & \multirow{2}{*}{$\begin{array}{l}\text { Erreur } \\
\text { relative } \\
(\mathrm{ET} / \mathrm{p})\end{array}$} & \multicolumn{2}{|c|}{ Intervalle de confiance (IC) } \\
\hline & & & & & $p-2 E T$ & $p+2 E T$ \\
\hline Enfants de 5-17 ans & 0,293 & 0,034 & 2176508 & 0,116 & 0,215 & 0,371 \\
\hline Enfants de 5 ans & 0,767 & 0,030 & 186498 & 0,039 & 0,705 & 0,828 \\
\hline Enfants de 6-11 ans & 0,299 & 0,052 & 1166140 & 0,172 & 0,177 & 0,421 \\
\hline Enfants de $12-13$ ans & 0,121 & 0,024 & 325361 & 0,194 & 0,069 & 0,174 \\
\hline Enfants de 14-17 ans & 0,212 & 0,042 & 498509 & 0,197 & 0,116 & 0,309 \\
\hline Garçons & 0,205 & 0,018 & 1069182 & 0,089 & 0,168 & 0,243 \\
\hline Filles & 0,377 & 0,064 & 1107325 & 0,170 & 0,225 & 0,530 \\
\hline Enfants des ménages avec moins de 50 USD & 0,334 & 0,043 & 1310114 & 0,129 & 0,223 & 0,446 \\
\hline Enfants des ménages avec 200 USD et plus & 0,138 & 0,040 & 38581 & 0,293 & 0,049 & 0,226 \\
\hline CM sans instruction & 0,314 & 0,032 & 1126907 & 0,102 & 0,246 & 0,382 \\
\hline CM de niveau secondaire et plus & 0,255 & 0,051 & 814812 & 0,201 & 0,129 & 0,381 \\
\hline Enfants du chef de ménage & 0,271 & 0,034 & 1683412 & 0,124 & 0,196 & 0,345 \\
\hline
\end{tabular}

Tableau des erreurs de sondage, Echantillon province du Kasaï Occidental

\begin{tabular}{|c|c|c|c|c|c|c|}
\hline \multirow{2}{*}{$\begin{array}{c}\text { Kasaï Occidental } \\
\text { Variables }\end{array}$} & \multirow{2}{*}{$\begin{array}{c}\text { Valeur } \\
p\end{array}$} & \multirow{2}{*}{$\begin{array}{l}\text { Erreur } \\
\text { type } \\
\text { (ET) }\end{array}$} & \multirow{2}{*}{$\begin{array}{l}\text { Effectif }(\mathrm{N}) \\
\text { pondéré }\end{array}$} & \multirow{2}{*}{$\begin{array}{l}\text { Erreur } \\
\text { relative } \\
(\mathrm{ET} / \mathrm{p}) \\
\end{array}$} & \multicolumn{2}{|c|}{ Intervalle de confiance (IC) } \\
\hline & & & & & $p-2 E T$ & $p+2 E T$ \\
\hline Enfants de 5-17 ans & 0,324 & 0,024 & 1461530 & 0,075 & 0,273 & 0,375 \\
\hline Enfants de 5 ans & 0,882 & 0,033 & 166133 & 0,038 & 0,814 & 0,950 \\
\hline Enfants de 6-11 ans & 0,320 & 0,027 & 762148 & 0,085 & 0,264 & 0,376 \\
\hline Enfants de $12-13$ ans & 0,104 & 0,022 & 220333 & 0,214 & 0,058 & 0,151 \\
\hline Enfants de $14-17$ ans & 0,192 & 0,041 & 312916 & 0,214 & 0,101 & 0,283 \\
\hline Garçons & 0,271 & 0,020 & 741130 & 0,075 & 0,229 & 0,313 \\
\hline Filles & 0,379 & 0,033 & 720415 & 0,088 & 0,308 & 0,449 \\
\hline Enfants des ménages avec moins de 50 USD & 0,353 & 0,025 & 919903 & 0,070 & 0,302 & 0,405 \\
\hline Enfants des ménages avec 200 USD et plus & 0,173 & 0,044 & 23586 & 0,255 & 0,073 & 0,273 \\
\hline CM sans instruction & 0,346 & 0,033 & 942064 & 0,095 & 0,276 & 0,416 \\
\hline CM de niveau secondaire & 0,265 & 0,022 & 391604 & 0,083 & 0,220 & 0,310 \\
\hline Enfants du chef de ménage & 0,310 & 0,023 & 1223743 & 0,074 & 0,262 & 0,358 \\
\hline
\end{tabular}




\section{Annexe 2 : Formule de calcul de l'intervalle de confiance de la différence de deux proportions}

Pour des grands échantillons, comme c'est le cas de l'enquête EADE-RDC, l'intervalle de confiance à $95 \%$ pour la différence entre deux proportions $\left(P_{1}\right.$ et $\left.P_{2}\right)$, peut s'obtenir par la formule suivante (Wonnacott et Wonnacott, 1991) :

$T=\left(P_{1}-P_{2}\right)= \pm 1,96 \sqrt{\frac{P_{1}\left(1-P_{1}\right)}{n_{1}}+\frac{P_{2}\left(1-P_{2}\right)}{n_{2}}} ;$ où 1,96 est la valeur de la loi normale pour un seuil $\alpha$ de $5 \%, n_{1}$ et $n_{2}$ étant l'effectif de chaque sous-population. Si l'intervalle de confiance de la différence des deux proportions ne comprend pas la valeur zéro, cela signifie que les deux proportions sont statistiquement différentes au seuil de $5 \%$.

En appliquant cette formule aux proportions d'EADE estimées en 2007, 2010 et 2012, on obtient les résultats suivants : aucun des intervalles de confiance des différences de proportions ne comprend 0.

\begin{tabular}{|c|c|c|c|c|c|c|c|}
\hline $\mathrm{n}_{1}$ & $\mathrm{~N}_{2}$ & $\mathrm{P}_{1}$ & $\mathrm{P}_{2}$ & $\mathrm{P}_{1}-\mathrm{P}_{2}$ & $T$ & Borne inf. & Borne sup. \\
\hline 16698 & 22012 & 0,385 & 0,325 & 0,06 & 0,010 & 0,050 & 0,070 \\
\hline $\mathrm{N}_{2}$ & $\mathrm{~N}_{3}$ & $\mathrm{P}_{2}$ & $P_{3}$ & $\mathrm{P}_{2}-\mathrm{P}_{3}$ & $\mathrm{~T}$ & Borne inf. & Borne sup. \\
\hline 22012 & 24042 & 0,325 & 0,289 & 0,036 & 0,008 & 0,028 & 0,044 \\
\hline $\mathrm{n}_{1}$ & $\mathrm{n}_{3}$ & $P_{1}$ & $\mathrm{P}_{3}$ & $\mathrm{P}_{1}-\mathrm{P}_{3}$ & $\mathrm{~T}$ & Borne inf. & Borne sup. \\
\hline 16698 & 24042 & 0,385 & 0,289 & 0,096 & 0,009 & 0,087 & 0,105 \\
\hline
\end{tabular}

Note : P1 = proportion des EADE de 5-17 ans en 2007, P2 = proportion des EADE de 5-17 ans en 2010 ; P3 = proportion des EADE de 5-17 ans en 2012

n1 = effectif non pondéré des enfants de 5-17 ans en 2007, n2 = effectif non pondéré des enfants de 5-17 ans en $2010 ; n 3=$ effectif non pondéré des enfants de 5-17 ans en 2012. 


\section{Annexe 3 : Notes sur la régression logistique et la hiérarchisation des déterminants de l'exclusion scolaire}

\section{Régression logistique}

L'un des objectifs de la recherche est d'évaluer le poids respectif de chacun des facteurs intervenant dans l'explication de la situation des EADE. Pour répondre à un tel objectif, il faut nécessairement procéder à des analyses explicatives multivariées qui permettent d'identifier l'effet net de chaque facteur et de pouvoir ainsi mettre en évidence les variables les plus déterminantes. La variable que nous expliquons (variable dépendante) est le risque que l'enfant soit en dehors de l'école (codé 1) ou à l'école (codé 0 ). La variable dépendante étant dichotomique, une des méthodes d'analyse statistique adaptée est la régression logistique. Dans la régression logistique une variable dépendante de nature dichotomique (prenant les valeurs 1 ou 0 ) est expliquée par un ensemble de variables qui peuvent être qualitatives ou quantitatives. Ce qui est modélisé dans la régression logistique, c'est la quantité appelée odds et qui est le rapport de deux probabilités. La probabilité que l'événement survienne $(P)$ et la probabilité que celui-ci ne survienne pas $(1-P)$. En considérant Xi variables indépendantes ( $\mathrm{i}$ allant de 1 à $\mathrm{k}$ ), l'équation de régression logistique peut s'écrire :

$\frac{P}{1-P}=e^{B_{0}+B_{1} X_{1}+\ldots+B_{k} X_{k}}=e^{B_{0}} \prod_{i=1}^{i=k} e^{B_{i} X_{i}}$

où les $\mathrm{Bi}$ représentent les coefficients de régression ; le facteur par lequel le odds change lorsque la $i^{\text {ème }}$ variable indépendante passe de la valeur 0 à la valeur 1 .

Dans la comparaison de deux sous-populations ou catégories d'une variable indépendante (dans lesquelles la probabilité que l'événement survienne est respectivement de $P_{1}$ et $P_{2}$ ), ce qui est examiné c'est le rapport

$$
\frac{\frac{P_{2}}{1-P_{2}}}{\frac{P_{1}}{1-P_{1}}}
$$

appelé rapport des chances ou odds ratio en anglais. Le rapport des chances s'interprète en termes d'écart par rapport à une modalité de référence. Par exemple, quel est le risque pour une fille d'être en dehors de l'école comparativement à un garçon ?

\section{Hiérarchisation des déterminants de l'exclusion scolaire}

Après l'analyse de l'effet des différents facteurs qui jouent sur les risques d'être en dehors de l'école, il convient de procéder à une hiérarchisation de ces facteurs ou déterminants, ce qui pourrait aider à opérer des choix en termes de priorité d'action.

Cette hiérarchisation des variables (déterminants) dans les différents modèles d'analyse peut se faire à partir du calcul de la contribution de chacune des variables à l'explication du phénomène des enfants et adolescents en dehors de l'école, mesurée par la statistique du Khi-2 du modèle. Cette contribution est donnée par la formule suivante :

$$
\text { Contribution }(\%)=\frac{X_{\text {final }}^{z}-X_{\text {modele sans la variable }}^{z}}{X_{\text {final }}^{2}} \times 100
$$


où $X^{2}$ représente la statistique du Khi-2. A partir de ces calculs, on peut bien mettre en évidence les variables qui contribuent le plus en examinant le Khi-2 du modèle avec l'ensemble des variables (modèle global ou modèle final) et celui du modèle sans la variable en question. La hiérarchisation conduit alors à ordonner les variables en fonction du pourcentage de Khi-2 qu'elles apportent à l'explication du phénomène des enfants et adolescents en dehors de l'école (de 1 à 10 en fonction du niveau d'importance décroissant des variables).

A titre d'illustration, considérons le modèle de régression logistique dont la variable dépendante est la situation de l'enfant par rapport à l'école (enfant en dehors de l'école codé 1 et enfant à l'école codé 0 ) pour les 12-17 ans, et les variables indépendantes:

$\checkmark$ sexe de l'enfant;

$\checkmark$ statut de l'enfant

$\checkmark$ lien de parenté avec le chef de ménage;

$\checkmark$ instruction du chef de ménage

$\checkmark$ sexe du chef de ménage

$\checkmark$ religion du chef de ménage;

$\checkmark$ distance par rapport à l'école secondaire

$\checkmark$ revenu

$\checkmark$ nombre d'enfants de 6-17 ans

$\checkmark$ nombre de malades dans le ménage.

En mettant l'ensemble de ces variables dans le modèle de régression logistique d'ensemble du groupe 12-17 ans (pour la RDC), on obtient un Khi-2 de 625,2. A supposer que l'on veuille calculer la contribution de la variable sexe de l'enfant à l'explication de la situation des enfants en rapport avec l'école. II nous suffit de retirer ladite variable du modèle général et l'on obtient un khi-2 de 549,7. La différence $(625,2-549,7=75,5)$ donne la contribue absolue de la variable sexe de l'enfant. La contribution relative (\%) est donnée par $75,5 / 625,2^{*} 100=12,1 \%$. On procède par la même méthode au calcul des contributions des autres variables. La valeur absolue de chaque contribution est utilisée pour la classification des différentes variables. En procédant ainsi, on obtient les résultats suivants qui donnent l'illustration pour la hiérarchisation au niveau de l'ensemble du pays.

Tableau de la hiérarchisation des déterminants pour l'ensemble du pays pour les 12-17 ans

\begin{tabular}{lccc}
\hline \multirow{2}{*}{ Variables } & \multicolumn{3}{c}{ Khi-2=625,2 } \\
\cline { 2 - 4 } & Khi-2 (sans la variable) & Contribution avec le Khi-2 & Valeur absolue \\
\hline Instruction CM & 525,8 & 15,9 & 15,9 \\
Revenu & 546 & 12,7 & 12,7 \\
Sexe de l'enfant & 549,7 & 12,1 & 12,1 \\
Distance école secondaire & 568,7 & 9,0 & 9,0 \\
Statut d'orphelinat & 577,13 & 7,7 & 7,7 \\
Lien de parenté avec CM & 668,1 & $-6,9$ & 6,9 \\
Sexe CM & 616,7 & 1,4 & 1,4 \\
Religion CM & 618 & 1,2 & 1,2 \\
Nombre de malade dans le ménage & 620,8 & 0,7 & 0,7 \\
Nombre d'enfant de 6-17 ans & 624,5 & 0,1 & 0,1 \\
\hline
\end{tabular}




\section{Annexe 4 : Les tableaux}

Tableau A1 : Valeur $r$ et répartition de l'échantillon EADE-RDC par strate

\begin{tabular}{|c|c|c|c|c|c|c|c|c|c|c|}
\hline & $\begin{array}{c}\text { Valeur } r \\
\text { (MICS 2010) }\end{array}$ & $\begin{array}{l}\text { Echantillon } \\
\text { Ménages }\end{array}$ & $\begin{array}{c}\text { Nombre de } \\
\text { Grappes }\end{array}$ & $\begin{array}{c}\text { Echantillon } \\
3-5 \text { ans }\end{array}$ & $\begin{array}{c}\text { Echantillon } \\
4-5 \text { ans }\end{array}$ & $\begin{array}{c}\text { Echantillon } \\
6-11 \text { ans }\end{array}$ & $\begin{array}{c}\text { Echantillon } \\
12-13 \text { ans }\end{array}$ & $\begin{array}{c}\text { Echantillon } \\
14-17 \text { ans }\end{array}$ & $\begin{array}{c}\text { Enfants } \\
12-17 \text { ans }\end{array}$ & $\begin{array}{c}\text { Total enfants } \\
3-17 \text { ans }\end{array}$ \\
\hline PAYS & & 13611 & 453 & 7546 & 4850 & 13245 & 3995 & 6083 & 10076 & 30869 \\
\hline Urbain & 0,175 & 5647 & 188 & 3078 & 2023 & 5951 & 1852 & 3030 & 4880 & 13911 \\
\hline Rural & 0,123 & 7964 & 265 & 4468 & 2827 & 7294 & 2143 & 3053 & 5196 & 16958 \\
\hline Kinshasa & 0,198 & 1146 & 38 & 518 & 358 & 1102 & 352 & 656 & 1007 & 2468 \\
\hline Bas Congo & 0,075 & 1139 & 38 & 486 & 321 & 944 & 289 & 384 & 673 & 2102 \\
\hline Urbain & 0,075 & 377 & 13 & 141 & 90 & 295 & 100 & 146 & 245 & 681 \\
\hline Rural & 0,128 & 762 & 25 & 345 & 231 & 649 & 189 & 238 & 428 & 1421 \\
\hline Bandundu & 0,024 & 1117 & 37 & 647 & 422 & 1030 & 286 & 485 & 771 & 2448 \\
\hline Urbain & 0,147 & 400 & 13 & 248 & 171 & 403 & 103 & 205 & 308 & 959 \\
\hline Rural & 0,129 & 717 & 24 & 399 & 251 & 627 & 183 & 280 & 463 & 1489 \\
\hline Equateur & 0,082 & 1031 & 34 & 640 & 396 & 1109 & 357 & 425 & 783 & 2532 \\
\hline Urbain & 0,136 & 394 & 13 & 239 & 146 & 452 & 139 & 175 & 315 & 1006 \\
\hline Rural & 0,167 & 637 & 21 & 401 & 250 & 657 & 218 & 250 & 468 & 1526 \\
\hline Province orientale & 0,135 & 1097 & 37 & 585 & 376 & 1053 & 336 & 500 & 836 & 2475 \\
\hline Urbain & 0,172 & 473 & 16 & 283 & 162 & 475 & 159 & 243 & 402 & 1160 \\
\hline Rural & 0,154 & 624 & 21 & 302 & 214 & 578 & 177 & 257 & 434 & 1315 \\
\hline Nord Kivu & 0,103 & 1411 & 47 & 910 & 547 & 1598 & 494 & 776 & 1276 & 3778 \\
\hline Urbain & 0,165 & 524 & 17 & 298 & 168 & 627 & 201 & 336 & 538 & 1462 \\
\hline Rural & 0,256 & 887 & 30 & 612 & 379 & 971 & 293 & 440 & 738 & 2316 \\
\hline Maniema & 0,140 & 943 & 32 & 461 & 305 & 745 & 208 & 318 & 526 & 1732 \\
\hline Urbain & 0,287 & 109 & 4 & 125 & 81 & 192 & 63 & 114 & 177 & 494 \\
\hline Rural & 0,099 & 834 & 28 & 336 & 224 & 553 & 145 & 204 & 349 & 1238 \\
\hline Sud Kivu & 0,074 & 1238 & 41 & 868 & 532 & 1264 & 440 & 630 & 1075 & 3202 \\
\hline Urbain & 0,105 & 493 & 16 & 322 & 202 & 597 & 211 & 320 & 532 & 1450 \\
\hline Rural & 0,202 & 745 & 25 & 546 & 330 & 667 & 229 & 310 & 543 & 1752 \\
\hline Katanga & 0,207 & 1905 & 64 & 1014 & 655 & 1759 & 522 & 751 & 1267 & 4047 \\
\hline Urbain & 0,200 & 717 & 24 & 405 & 265 & 735 & 225 & 285 & 510 & 1651 \\
\hline Rural & 0,275 & 1188 & 40 & 609 & 390 & 1024 & 297 & 466 & 757 & 2396 \\
\hline Kassaï oriental & 0,161 & 1233 & 41 & 687 & 445 & 1275 & 353 & 544 & 895 & 2860 \\
\hline Urbain & 0,327 & 560 & 19 & 312 & 193 & 592 & 175 & 302 & 478 & 1381 \\
\hline Rural & 0,167 & 673 & 22 & 375 & 252 & 683 & 178 & 242 & 417 & 1479 \\
\hline Kassaï occidental & 0,229 & 1351 & 45 & 783 & 490 & 1364 & 358 & 613 & 968 & 3119 \\
\hline Urbain & 0,117 & 454 & 15 & 275 & 186 & 480 & 125 & 247 & 369 & 1127 \\
\hline Rural & 0,166 & 897 & 30 & 508 & 304 & 884 & 233 & 366 & 599 & 1992 \\
\hline
\end{tabular}


Tableau A2 : Statut scolaire des enfants de 5-17 ans par âge (en \%)

\begin{tabular}{|c|c|c|c|c|c|c|c|}
\hline \multirow[b]{2}{*}{ Ages } & \multicolumn{5}{|c|}{ Statut scolaire } & \multicolumn{2}{|c|}{ EADE } \\
\hline & Scolarisé & Déscolarisé & $\begin{array}{c}\text { Jamais } \\
\text { fréquenté }\end{array}$ & Total & Effectifs & $\%$ & Effectifs \\
\hline 5 & 21,2 & 0,5 & 78,3 & 100,0 & 2720081 & 78,8 & 2144194 \\
\hline 6 & 47,4 & 1,1 & 51,5 & 100,0 & 2473366 & 52,6 & 1302038 \\
\hline 7 & 67,3 & 3,3 & 29,4 & 100,0 & 2445321 & 32,7 & 799833 \\
\hline 8 & 76,6 & 4,9 & 18,5 & 100,0 & 2306737 & 23,4 & 540600 \\
\hline 9 & 85,1 & 3,9 & 11,0 & 100,0 & 2009033 & 14,9 & 299820 \\
\hline 10 & 84,2 & 7,0 & 8,8 & 100,0 & 2065710 & 15,8 & 326743 \\
\hline 11 & 86,8 & 6,1 & 7,1 & 100,0 & 1817431 & 13,2 & 240217 \\
\hline 12 & 86,9 & 6,8 & 6,2 & 100,0 & 2353144 & 13,1 & 307511 \\
\hline 13 & 86,9 & 7,3 & 5,8 & 100,0 & 1564801 & 13,1 & 205656 \\
\hline 14 & 85,1 & 10,9 & 4,0 & 100,0 & 1693909 & 14,9 & 253236 \\
\hline 15 & 81,9 & 11,7 & 6,4 & 100,0 & 1563549 & 18,1 & 282858 \\
\hline 16 & 74,4 & 19,4 & 6,3 & 100,0 & 1271109 & 25,6 & 326006 \\
\hline 17 & 70,4 & 23,9 & 5,7 & 100,0 & 1171090 & 29,6 & 347162 \\
\hline Total & 71,0 & 6,8 & 22,1 & 100,0 & 25455281 & 28,9 & 7375874 \\
\hline
\end{tabular}

Source : Données de l'enquête auprès des ménages, EADE-RDC 2012 
Tableau A3 : Statut scolaire des enfants de 5-17 ans par âge et sexe (en \%)

\begin{tabular}{|c|c|c|c|c|c|c|c|}
\hline \multirow[b]{2}{*}{ Ages } & \multicolumn{5}{|c|}{ Statut scolaire } & \multicolumn{2}{|c|}{ EADE } \\
\hline & Scolarisé & Déscolarisé & $\begin{array}{c}\text { Jamais } \\
\text { fréquenté }\end{array}$ & Total & Effectifs & $\%$ & Effectifs \\
\hline \multicolumn{8}{|c|}{ Garçons } \\
\hline 5 & 20,7 & 0,6 & 78,7 & 100,0 & 1376623 & 79,3 & 1091942 \\
\hline 6 & 48,8 & 0,7 & 50,6 & 100,0 & 1313887 & 51,2 & 673329 \\
\hline 7 & 69,3 & 3,3 & 27,3 & 100,0 & 1303391 & 30,7 & 399944 \\
\hline 8 & 76,8 & 5,6 & 17,6 & 100,0 & 1156609 & 23,2 & 268660 \\
\hline 9 & 85,8 & 3,5 & 10,7 & 100,0 & 1057309 & 14,2 & 150132 \\
\hline 10 & 85,1 & 7,2 & 7,7 & 100,0 & 1052667 & 14,9 & 157316 \\
\hline 11 & 88,9 & 5,0 & 6,1 & 100,0 & 907641 & 11,1 & 100409 \\
\hline 12 & 90,7 & 5,2 & 4,1 & 100,0 & 1244331 & 9,3 & 115582 \\
\hline 13 & 89,1 & 6,9 & 4,0 & 100,0 & 803005 & 10,9 & 87826 \\
\hline 14 & 89,0 & 8,1 & 2,9 & 100,0 & 896099 & 11,0 & 98717 \\
\hline 15 & 84,3 & 11,0 & 4,8 & 100,0 & 841977 & 15,7 & 132576 \\
\hline 16 & 82,5 & 13,0 & 4,5 & 100,0 & 649033 & 17,5 & 113464 \\
\hline 17 & 83,2 & 15,1 & 1,7 & 100,0 & 555954 & 16,8 & 93513 \\
\hline Total & 73,5 & 5,6 & 20,9 & 100,0 & 13158526 & 26,5 & 3483410 \\
\hline \multicolumn{8}{|l|}{ Filles } \\
\hline 5 & 21,7 & 0,4 & 77,9 & 100,0 & 1343458 & 78,3 & 1052252 \\
\hline 6 & 45,8 & 1,6 & 52,6 & 100,0 & 1159479 & 54,2 & 628709 \\
\hline 7 & 65,0 & 3,2 & 31,8 & 100,0 & 1141930 & 35,0 & 399889 \\
\hline 8 & 76,4 & 4,3 & 19,4 & 100,0 & 1150128 & 23,6 & 271940 \\
\hline 9 & 84,3 & 4,5 & 11,3 & 100,0 & 951724 & 15,7 & 149688 \\
\hline 10 & 83,3 & 6,8 & 9,9 & 100,0 & 1013043 & 16,7 & 169427 \\
\hline 11 & 84,6 & 7,2 & 8,2 & 100,0 & 909790 & 15,4 & 139808 \\
\hline 12 & 82,7 & 8,7 & 8,6 & 100,0 & 1108813 & 17,3 & 191929 \\
\hline 13 & 84,5 & 7,8 & 7,7 & 100,0 & 761796 & 15,5 & 117830 \\
\hline 14 & 80,6 & 14,1 & 5,3 & 100,0 & 797810 & 19,4 & 154519 \\
\hline 15 & 79,2 & 12,5 & 8,3 & 100,0 & 721572 & 20,8 & 150282 \\
\hline 16 & 65,8 & 26,0 & 8,1 & 100,0 & 622076 & 34,2 & 212542 \\
\hline 17 & 58,8 & 31,9 & 9,4 & 100,0 & 615136 & 41,2 & 253649 \\
\hline Total & 68,3 & 8,2 & 23,5 & 100,0 & 12296755 & 31,7 & 3892464 \\
\hline
\end{tabular}


Tableau A4 : Statut scolaire des enfants de 5-17 ans par âge et Milieu de résidence (en \%)

\begin{tabular}{|c|c|c|c|c|c|c|c|}
\hline \multirow[b]{2}{*}{ Ages } & \multicolumn{5}{|c|}{ Statut scolaire } & \multicolumn{2}{|c|}{ EADE } \\
\hline & Scolarisé & Déscolarisé & $\begin{array}{c}\text { Jamais } \\
\text { fréquenté }\end{array}$ & Total & Effectifs & $\%$ & Effectifs \\
\hline \multicolumn{8}{|l|}{ Urbain } \\
\hline 5 & 40,4 & 1,3 & 58,3 & 100,0 & 762974 & 59,6 & 454494 \\
\hline 6 & 68,2 & 1,8 & 30,0 & 100,0 & 728380 & 31,8 & 231263 \\
\hline 7 & 76,4 & 5,5 & 18,1 & 100,0 & 778185 & 23,6 & 183683 \\
\hline 8 & 84,7 & 6,1 & 9,2 & 100,0 & 747769 & 15,3 & 114694 \\
\hline 9 & 88,5 & 5,3 & 6,2 & 100,0 & 631987 & 11,5 & 72775 \\
\hline 10 & 88,2 & 9,1 & 2,8 & 100,0 & 660718 & 11,8 & 78161 \\
\hline 11 & 87,8 & 7,8 & 4,4 & 100,0 & 655011 & 12,2 & 79814 \\
\hline 12 & 89,7 & 7,4 & 2,9 & 100,0 & 729242 & 10,3 & 75064 \\
\hline 13 & 91,5 & 6,2 & 2,3 & 100,0 & 516475 & 8,5 & 43895 \\
\hline 14 & 87,6 & 11,8 & 0,7 & 100,0 & 583201 & 12,4 & 72513 \\
\hline 15 & 89,2 & 8,6 & 2,2 & 100,0 & 605022 & 10,8 & 65442 \\
\hline 16 & 82,1 & 17,0 & 1,0 & 100,0 & 487295 & 17,9 & 87321 \\
\hline 17 & 76,2 & 22,9 & 1,0 & 100,0 & 512828 & 23,8 & 122232 \\
\hline Total & 80,0 & 7,9 & 12,1 & 100,0 & 8399087 & 20,0 & 1681351 \\
\hline \multicolumn{8}{|l|}{ Rural } \\
\hline 5 & 13,7 & 0,2 & 86,1 & 100,0 & 1957109 & 86,3 & 1689701 \\
\hline 6 & 38,6 & 0,8 & 60,5 & 100,0 & 1744987 & 61,4 & 1070776 \\
\hline 7 & 63,0 & 2,2 & 34,7 & 100,0 & 1667137 & 37,0 & 616151 \\
\hline 8 & 72,7 & 4,4 & 23,0 & 100,0 & 1558968 & 27,3 & 425906 \\
\hline 9 & 83,5 & 3,3 & 13,2 & 100,0 & 1377044 & 16,5 & 227044 \\
\hline 10 & 82,3 & 6,0 & 11,6 & 100,0 & 1404991 & 17,7 & 248582 \\
\hline 11 & 86,2 & 5,1 & 8,7 & 100,0 & 1162419 & 13,8 & 160402 \\
\hline 12 & 85,7 & 6,6 & 7,7 & 100,0 & 1623902 & 14,3 & 232447 \\
\hline 13 & 84,6 & 7,9 & 7,5 & 100,0 & 1048327 & 15,4 & 161761 \\
\hline 14 & 83,7 & 10,5 & 5,8 & 100,0 & 1110707 & 16,3 & 180722 \\
\hline 15 & 77,3 & 13,7 & 9,0 & 100,0 & 958527 & 22,7 & 217417 \\
\hline 16 & 69,5 & 20,9 & 9,6 & 100,0 & 783814 & 30,5 & 238685 \\
\hline 17 & 65,8 & 24,7 & 9,4 & 100,0 & 658261 & 34,2 & 224929 \\
\hline Total & 66,6 & 6,3 & 27,1 & 100,0 & 17056194 & 33,4 & 5694523 \\
\hline
\end{tabular}


Tableau A5 : Distribution (en \%) des enfants en âge scolaire (5-17 ans) selon le statut scolaire par sexe et par province

\begin{tabular}{|c|c|c|c|c|c|c|c|}
\hline \multirow[b]{2}{*}{ Caractéristiques } & \multicolumn{5}{|c|}{ Statut scolaire } & \multicolumn{2}{|c|}{ EADE } \\
\hline & Scolarisé & Déscolarisé & $\begin{array}{l}\text { Jamais } \\
\text { fréquenté }\end{array}$ & Total & Effectifs & $\%$ & Effectifs \\
\hline \multicolumn{8}{|l|}{ Garçons } \\
\hline Kinshasa & 84,1 & 8,2 & 7,6 & 100,0 & 1635573 & 15,8 & 259584 \\
\hline Bas-Congo & 76,5 & 4,3 & 19,2 & 100,0 & 763887 & 23,5 & 179672 \\
\hline Bandundu & 80,2 & 1,9 & 17,9 & 100,0 & 1443036 & 19,8 & 285128 \\
\hline Equateur & 74,7 & 3,3 & 22,0 & 100,0 & 1344817 & 25,3 & 339739 \\
\hline Orientale & 69,4 & 8,6 & 21,9 & 100,0 & 1728310 & 30,5 & 528466 \\
\hline Nord Kivu & 58,2 & 8,9 & 33,0 & 100,0 & 1151835 & 41,9 & 481984 \\
\hline Maniema & 75,0 & 2,5 & 22,5 & 100,0 & 381478 & 25,0 & 95196 \\
\hline sud Kivu & 72,8 & 6,5 & 20,8 & 100,0 & 859171 & 27,3 & 234096 \\
\hline Katanga & 67,7 & 6,3 & 26,0 & 100,0 & 2040108 & 32,3 & 659190 \\
\hline Kasaï oriental & 79,5 & 2,7 & 17,8 & 100,0 & 1069192 & 20,5 & 219591 \\
\hline Kasaï occidental & 72,9 & 3,2 & 23,9 & 100,0 & 741119 & 27,1 & 200766 \\
\hline Total & 73,5 & 5,6 & 20,9 & 100,0 & 13158526 & 26,5 & 3483412 \\
\hline \multicolumn{8}{|l|}{ Filles } \\
\hline Kinshasa & 84,4 & 8,9 & 6,7 & 100,0 & 1634931 & 15,6 & 255068 \\
\hline Bas-Congo & 68,6 & 7,4 & 24,0 & 100,0 & 641623 & 31,4 & 201446 \\
\hline Bandundu & 78,7 & 2,4 & 18,9 & 100,0 & 1368949 & 21,3 & 291420 \\
\hline Equateur & 69,2 & 5,6 & 25,2 & 100,0 & 1254013 & 30,8 & 386455 \\
\hline Orientale & 65,9 & 9,4 & 24,7 & 100,0 & 1499657 & 34,1 & 511392 \\
\hline Nord Kivu & 53,9 & 10,6 & 35,6 & 100,0 & 1110580 & 46,2 & 512382 \\
\hline Maniema & 73,0 & 4,6 & 22,4 & 100,0 & 338752 & 27,0 & 91625 \\
\hline sud Kivu & 66,5 & 8,0 & 25,5 & 100,0 & 826933 & 33,5 & 276673 \\
\hline Katanga & 62,3 & 9,5 & 28,1 & 100,0 & 1793584 & 37,6 & 675686 \\
\hline Kasaï oriental & 62,3 & 12,1 & 25,6 & 100,0 & 1107317 & 37,7 & 417628 \\
\hline Kasaï occidental & 62,1 & 8,6 & 29,2 & 100,0 & 720411 & 37,8 & 272686 \\
\hline Total & 68,3 & 8,2 & 23,5 & 100,0 & 12296750 & 31,7 & 3892461 \\
\hline \multicolumn{8}{|l|}{ Ensemble } \\
\hline Kinshasa & 84,3 & 8,6 & 7,2 & 100,0 & 3270504 & 15,8 & 514652 \\
\hline Bas-Congo & 72,9 & 5,7 & 21,4 & 100,0 & 1405510 & 27,1 & 381118 \\
\hline Bandundu & 79,5 & 2,1 & 18,4 & 100,0 & 2811985 & 20,5 & 576548 \\
\hline Equateur & 72,1 & 4,4 & 23,5 & 100,0 & 2598830 & 27,9 & 726194 \\
\hline Orientale & 67,8 & 9,0 & 23,2 & 100,0 & 3227967 & 32,2 & 1039858 \\
\hline Nord Kivu & 56,0 & 9,7 & 34,2 & 100,0 & 2262415 & 43,9 & 994366 \\
\hline Maniema & 74,1 & 3,5 & 22,5 & 100,0 & 720230 & 26,0 & 186821 \\
\hline Sud Kivu & 69,7 & 7,2 & 23,1 & 100,0 & 1686104 & 30,3 & 510769 \\
\hline Katanga & 65,2 & 7,8 & 27,0 & 100,0 & 3833692 & 34,8 & 1334876 \\
\hline Kasaï oriental & 70,7 & 7,5 & 21,8 & 100,0 & 2176509 & 29,3 & 637219 \\
\hline Kasaï occidental & 67,6 & 5,9 & 26,5 & 100,0 & 1461530 & 32,4 & 473452 \\
\hline Total & 71,1 & 6,8 & 22,1 & 100,0 & 25455276 & 28,9 & 7375873 \\
\hline
\end{tabular}


Tableau A6 : Distribution (en \%) des enfants de 6-11 ans selon le statut scolaire par sexe et par province

\begin{tabular}{|c|c|c|c|c|c|c|c|}
\hline \multirow[b]{2}{*}{ Caractéristiques } & \multicolumn{5}{|c|}{ Statut scolaire } & \multicolumn{2}{|c|}{ EADE } \\
\hline & Scolarisé & Déscolarisé & $\begin{array}{c}\text { Jamais } \\
\text { fréquenté }\end{array}$ & Total & Effectifs & $\%$ & Effectifs \\
\hline \multicolumn{8}{|l|}{ Garçons } \\
\hline Kinshasa & 86,2 & 6,9 & 6,9 & 100,0 & 812752 & 13,8 & 111982 \\
\hline Bas-Congo & 78,5 & 1,3 & 20,3 & 100,0 & 391067 & 21,5 & 84412 \\
\hline Bandundu & 79,8 & 0,9 & 19,3 & 100,0 & 751750 & 20,2 & 152035 \\
\hline Equateur & 76,4 & 2,1 & 21,5 & 100,0 & 715193 & 23,6 & 168983 \\
\hline Orientale & 71,7 & 7,0 & 21,3 & 100,0 & 848198 & 28,3 & 240262 \\
\hline Nord Kivu & 59,8 & 6,1 & 34,1 & 100,0 & 601694 & 40,2 & 241392 \\
\hline Maniema & 80,3 & 1,0 & 18,7 & 100,0 & 182098 & 19,7 & 35722 \\
\hline sud Kivu & 76,2 & 3,6 & 20,2 & 100,0 & 442963 & 23,8 & 105441 \\
\hline Katanga & 65,8 & 4,8 & 29,4 & 100,0 & 1096185 & 34,2 & 375443 \\
\hline Kasaï oriental & 75,9 & 2,9 & 21,1 & 100,0 & 567576 & 24,1 & 136511 \\
\hline Kasaï occidental & 74,4 & 2,2 & 23,4 & 100,0 & 382028 & 25,6 & 97614 \\
\hline Total & 74,2 & 4,0 & 21,7 & 100,0 & 6791504 & 25,8 & 1749792 \\
\hline \multicolumn{8}{|l|}{ Filles } \\
\hline Kinshasa & 87,5 & 7,3 & 5,2 & 100,0 & 814158 & 12,5 & 101507 \\
\hline Bas-Congo & 76,6 & 2,7 & 20,7 & 100,0 & 310641 & 23,4 & 72821 \\
\hline Bandundu & 82,3 & 2,0 & 15,6 & 100,0 & 693760 & 17,7 & 122505 \\
\hline Equateur & 72,6 & 2,5 & 24,9 & 100,0 & 658974 & 27,4 & 180854 \\
\hline Orientale & 70,8 & 4,4 & 24,8 & 100,0 & 793102 & 29,2 & 232058 \\
\hline Nord Kivu & 59,6 & 3,9 & 36,6 & 100,0 & 537004 & 40,4 & 216585 \\
\hline Maniema & 76,2 & 2,0 & 21,8 & 100,0 & 182130 & 23,8 & 43162 \\
\hline sud Kivu & 68,8 & 5,1 & 26,1 & 100,0 & 417134 & 31,2 & 130153 \\
\hline Katanga & 68,0 & 5,4 & 26,7 & 100,0 & 940501 & 32,0 & 301317 \\
\hline Kasaï oriental & 64,5 & 5,6 & 29,9 & 100,0 & 598564 & 35,5 & 212335 \\
\hline Kasaï occidental & 61,6 & 4,7 & 33,7 & 100,0 & 380123 & 38,4 & 146157 \\
\hline Total & 72,2 & 4,4 & 23,4 & 100,0 & 6326091 & 27,8 & 1759459 \\
\hline \multicolumn{8}{|l|}{ Ensemble } \\
\hline Kinshasa & 86,9 & 7,1 & 6,0 & 100,0 & 1626910 & 13,1 & 213489 \\
\hline Bas-Congo & 77,6 & 1,9 & 20,4 & 100,0 & 701708 & 22,4 & 157233 \\
\hline Bandundu & 81,0 & 1,5 & 17,5 & 100,0 & 1445510 & 19,0 & 274540 \\
\hline Equateur & 74,5 & 2,3 & 23,1 & 100,0 & 1374167 & 25,5 & 349837 \\
\hline Orientale & 71,3 & 5,7 & 23,0 & 100,0 & 1641300 & 28,7 & 472320 \\
\hline Nord Kivu & 59,7 & 5,0 & 35,3 & 100,0 & 1138698 & 40,3 & 457977 \\
\hline Maniema & 78,3 & 1,5 & 20,2 & 100,0 & 364228 & 21,7 & 78884 \\
\hline Sud Kivu & 72,6 & 4,3 & 23,1 & 100,0 & 860097 & 27,4 & 235594 \\
\hline Katanga & 66,8 & 5,1 & 28,1 & 100,0 & 2036686 & 33,2 & 676760 \\
\hline Kasaï oriental & 70,1 & 4,3 & 25,6 & 100,0 & 1166140 & 29,9 & 348846 \\
\hline Kasaï occidental & 68,0 & 3,5 & 28,5 & 100,0 & 762151 & 32,0 & 243771 \\
\hline Total & 73,2 & 4,2 & 22,5 & 100,0 & 13117595 & 26,8 & 3509251 \\
\hline
\end{tabular}


Tableau A7 : Distribution (en \%) des enfants et adolescents de 6-13 ans selon la typologie des EADE par sexe, milieu de résidence par dimension

\begin{tabular}{|c|c|c|c|c|}
\hline \multirow{2}{*}{ Catégories des enfants non scolarisés } & \multicolumn{2}{|c|}{ Proportions } & \multicolumn{2}{|r|}{ Effectifs } \\
\hline & Dimension 2 & Dimension 3 & Dimension 2 & Dimension 3 \\
\hline \multicolumn{5}{|l|}{ Garçons } \\
\hline Enfants qui ont abandonné & 15,7 & 59,1 & 275159 & 120113 \\
\hline Enfants qui devraient entrer d'ici l'âge de 17 ans & 75,4 & 9,1 & 1319355 & 18547 \\
\hline Enfants qui n'entreront jamais & 8,8 & 31,8 & 154415 & 64591 \\
\hline Enfants non scolarisés - Total & 25,8 & 9,9 & 1748929 & 203250 \\
\hline \multicolumn{5}{|l|}{ Filles } \\
\hline Enfants qui ont abandonné & 16,0 & 50,3 & 281768 & 155887 \\
\hline Enfants qui devraient entrer d'ici l'âge de 17 ans & 72,8 & 16,8 & 1281225 & 51957 \\
\hline Enfants qui n'entreront jamais & 11,2 & 32,9 & 197358 & 102059 \\
\hline Enfants non scolarisés & 27,8 & 16,6 & 1760351 & 309903 \\
\hline \multicolumn{5}{|l|}{ Urbain } \\
\hline Enfants qui ont abandonné & 32,4 & 72,2 & 247317 & 85991 \\
\hline Enfants qui devraient entrer d'ici l'âge de 17 ans & 54,2 & 4,4 & 416187 & 5491 \\
\hline Enfants qui n'entreront jamais & 13,5 & 23,4 & 95495 & 27313 \\
\hline Enfants non scolarisés & 18,1 & 9,6 & 761219 & 119124 \\
\hline \multicolumn{5}{|l|}{ Rural } \\
\hline Enfants qui ont abandonné & 11,2 & 48,3 & 309610 & 190008 \\
\hline Enfants qui devraient entrer d'ici l'âge de 17 ans & 78,8 & 15,7 & 2184393 & 65013 \\
\hline Enfants qui n'entreront jamais & 10,0 & 36,0 & 256278 & 139337 \\
\hline Enfants non scolarisés & 30,8 & 14,7 & 2748061 & 394029 \\
\hline \multicolumn{5}{|l|}{ RDC } \\
\hline Enfants qui ont abandonné & 15,8 & 53,7 & 556927 & 275999 \\
\hline Enfants qui devraient entrer d'ici l'âge de 17 ans & 74,2 & 13,5 & 2600580 & 70504 \\
\hline Enfants qui n'entreront jamais & 10,0 & 32,8 & 351773 & 166650 \\
\hline Enfants non scolarisés & 26,7 & 13,0 & 3509280 & 513153 \\
\hline
\end{tabular}


Tableau A8: Distribution (en \%) des enfants de 5-17 ans en rupture familiale selon le statut scolaire par province

\begin{tabular}{|c|c|c|c|c|c|c|c|}
\hline \multirow[b]{2}{*}{ Provinces } & \multicolumn{5}{|c|}{ Statut scolaire } & \multicolumn{2}{|c|}{ EADE } \\
\hline & Scolarisé & Déscolarisé & $\begin{array}{l}\text { Jamais } \\
\text { fréquenté }\end{array}$ & Total & Effectifs & $\%$ & Effectifs \\
\hline \multicolumn{8}{|l|}{$\begin{array}{l}\text { Enfants dans les } \\
\text { structures }\end{array}$} \\
\hline Kinshasa & 77,5 & 15,3 & 7,2 & 100,0 & 2123 & 22,5 & 477 \\
\hline Bas-Congo & 79,8 & 6,9 & 13,3 & 100,0 & 173 & 20,2 & 35 \\
\hline Bandundu & 89,6 & 5,7 & 4,7 & 100,0 & 680 & 10,4 & 71 \\
\hline Equateur & 90,6 & 6,1 & 3,3 & 100,0 & 180 & 9,4 & 17 \\
\hline Province Orientale & 74,0 & 16,7 & 9,3 & 100,0 & 366 & 26,0 & 95 \\
\hline Nord-Kivu & 97,9 & 0,5 & 1,5 & 100,0 & 3770 & 2,1 & 78 \\
\hline Maniema & 88,9 & 9,3 & 1,9 & 100,0 & 216 & 11,1 & 24 \\
\hline Sud-Kivu & 72,2 & 17,9 & 9,9 & 100,0 & 2496 & 27,8 & 693 \\
\hline Katanga & 81,4 & 12,3 & 6,3 & 100,0 & 463 & 18,6 & 86 \\
\hline Kasaï Oriental & 65,2 & 20,2 & 14,6 & 100,0 & 526 & 34,8 & 183 \\
\hline Kasaï Occidental & 79,6 & 10,0 & 10,4 & 100,0 & 270 & 20,4 & 55 \\
\hline Ensemble & 83,9 & 10,0 & 6,1 & 100,0 & 11263 & 16,1 & 1814 \\
\hline \multicolumn{8}{|l|}{ Enfants de la rue } \\
\hline Kinshasa & 1,7 & 67,9 & 30,4 & 100,0 & 1532 & 98,3 & 1506 \\
\hline Bas-Congo & 3,1 & 63,6 & 33,3 & 100,0 & 129 & 96,9 & 125 \\
\hline Bandundu & 0,0 & 70,0 & 30,0 & 100,0 & 30 & 100,0 & 30 \\
\hline Equateur & 0,0 & 66,7 & 33,3 & 100,0 & 42 & 100,0 & 42 \\
\hline Province Orientale & 18,6 & 62,3 & 19,2 & 100,0 & 334 & 81,4 & 272 \\
\hline Nord-Kivu & 3,8 & 60,3 & 35,8 & 100,0 & 416 & 96,2 & 400 \\
\hline Maniema & 0,0 & 86,7 & 13,3 & 100,0 & 15 & 100,0 & 15 \\
\hline Sud-Kivu & 7,4 & 43,6 & 48,9 & 100,0 & 1077 & 92,6 & 997 \\
\hline Katanga & 1,9 & 56,6 & 41,5 & 100,0 & 159 & 98,1 & 156 \\
\hline Kasaï Oriental & 2,3 & 57,3 & 40,4 & 100,0 & 354 & 97,7 & 346 \\
\hline Kasaï Occidental & 0,4 & 57,8 & 41,9 & 100,0 & 270 & 99,6 & 269 \\
\hline Ensemble & 4,6 & 58,8 & 36,6 & 100,0 & 4358 & 95,4 & 4158 \\
\hline
\end{tabular}

Source : Données du recensement auprès des structures d'accueil des enfants, EADE-RDC 2012 
Tableau A9 : Répartition des effectifs d'enfants d'âges du pré-primaire en dehors de l'école par statut scolaire selon certaines caractéristiques

\begin{tabular}{|c|c|c|c|c|c|c|}
\hline \multirow[b]{2}{*}{ Caractéristiques } & \multicolumn{3}{|c|}{ Enfants de $3-5$ ans } & \multicolumn{3}{|c|}{ Enfants de 5 ans } \\
\hline & Déscolarisé & $\begin{array}{l}\text { Jamais } \\
\text { fréquenté }\end{array}$ & Ens. EADE & Déscolarisé & $\begin{array}{l}\text { Jamais } \\
\text { fréquenté }\end{array}$ & $\begin{array}{l}\text { Ens. } \\
\text { EADE }\end{array}$ \\
\hline \multicolumn{7}{|l|}{ Age } \\
\hline 3 & 3427 & 2365446 & 2368873 & na & na & na \\
\hline 4 & 4956 & 2181072 & 2186028 & na & na & na \\
\hline 5 & 13792 & 2130402 & 2144194 & 13792 & 2130402 & 2144194 \\
\hline Total & 22175 & 6676920 & 6699095 & 13792 & 2130402 & 2144194 \\
\hline \multicolumn{7}{|c|}{ Sexe du membre du ménage } \\
\hline Garçons & 12866 & 3440732 & 3453598 & 8181 & 1083761 & 1091942 \\
\hline Filles & 9310 & 3236188 & 3245498 & 5611 & 1046641 & 1052252 \\
\hline Total & 22176 & 6676920 & 6699096 & 13792 & 2130402 & 2144194 \\
\hline \multicolumn{7}{|l|}{ Milieu } \\
\hline Urbain & 14252 & 1691892 & 1706144 & 10061 & 444433 & 454494 \\
\hline Rural & 7924 & 4985028 & 4992952 & 3732 & 1685969 & 1689701 \\
\hline Total & 22176 & 6676920 & 6699096 & 13793 & 2130402 & 2144195 \\
\hline \multicolumn{7}{|l|}{ Province } \\
\hline Kinshasa & 5671 & 545056 & 550727 & 4435 & 128605 & 133040 \\
\hline Bas-Congo & 2197 & 424995 & 427192 & 1818 & 134150 & 135968 \\
\hline Bandundu & 279 & 781844 & 782123 & - & 233514 & 233514 \\
\hline Equateur & 3081 & 751908 & 754989 & 2594 & 248794 & 251388 \\
\hline Orientale & 2801 & 670009 & 672810 & - & 291038 & 291038 \\
\hline Nord Kivu & 294 & 734745 & 735039 & 228 & 270172 & 270400 \\
\hline Maniema & 794 & 203293 & 204087 & 458 & 76790 & 77248 \\
\hline sud Kivu & 1122 & 511297 & 512419 & - & 144191 & 144191 \\
\hline Katanga & 3284 & 1125332 & 1128616 & 3284 & 314562 & 317846 \\
\hline Kasaï oriental & 1244 & 494374 & 495618 & 975 & 142018 & 142993 \\
\hline Kasaï occidental & 1409 & 434067 & 435476 & - & 146569 & 146569 \\
\hline Total & 22176 & 6676920 & 6699096 & 13792 & 2130403 & 2144195 \\
\hline
\end{tabular}

Source : Données de l'enquête auprès des ménages, EADE-RDC 2012 na : non applicable 
Tableau A10: Répartition des effectifs d'enfants d'âges du pré-primaire en dehors de l'école par statut scolaire selon certaines caractéristiques

\begin{tabular}{|c|c|c|c|c|c|c|}
\hline \multirow[b]{2}{*}{ Caractéristiques } & \multicolumn{3}{|c|}{ Enfants de $3-5$ ans } & \multicolumn{3}{|c|}{ Enfants de 5 ans } \\
\hline & Déscolarisé & $\begin{array}{l}\text { Jamais } \\
\text { fréquenté }\end{array}$ & Ens. EADE & Déscolarisé & $\begin{array}{l}\text { Jamais } \\
\text { fréquenté }\end{array}$ & $\begin{array}{l}\text { Ens. } \\
\text { EADE }\end{array}$ \\
\hline \multicolumn{7}{|l|}{ Statut familial } \\
\hline Enfant du CM & 14231 & 5548594 & 5562825 & 6183 & 1782751 & 1788934 \\
\hline Autre parent & 7945 & 1125674 & 1133619 & 7610 & 347651 & 355261 \\
\hline Sans parenté & - & 2652 & 2652 & - & - & - \\
\hline Total & 22176 & 6676920 & 6699096 & 13793 & 2130402 & 2144195 \\
\hline \multicolumn{7}{|l|}{ statut d'orphelin } \\
\hline Non orphelin & 21014 & 6307020 & 6328034 & 12630 & 2001874 & 2014504 \\
\hline Orphelin de mère & - & 75964 & 75964 & - & 25078 & 25078 \\
\hline Orphelin de père & 1162 & 246298 & 247460 & 1162 & 90120 & 91282 \\
\hline Orphelin complet & - & 36065 & 36065 & - & 10277 & 10277 \\
\hline Total & 22176 & 6665347 & 6687523 & 13792 & 2127349 & 2141141 \\
\hline \multicolumn{7}{|c|}{ Niveau d'instruction du CM } \\
\hline Aucun & 16220 & 4636803 & 4653023 & 9654 & 1522061 & 1531715 \\
\hline Primaire & 2258 & 571518 & 573776 & 2258 & 181122 & 183380 \\
\hline Secondaire+ & 3697 & 1468600 & 1472297 & 1880 & 427219 & 429099 \\
\hline Total & 22175 & 6676921 & 6699096 & 13792 & 2130402 & 2144194 \\
\hline \multicolumn{7}{|c|}{ Sexe du chef de ménage } \\
\hline Hommes & 12030 & 3945738 & 3957768 & 8341 & 1262195 & 1270536 \\
\hline Femmes & 10146 & 2731182 & 2741328 & 5452 & 868207 & 873659 \\
\hline Total & 22176 & 6676920 & 6699096 & 13793 & 2130402 & 2144195 \\
\hline \multicolumn{7}{|c|}{ Revenu mensuel du ménage } \\
\hline Moins de 50 USD & 5411 & 3753062 & 3758473 & 2258 & 1244433 & 1246691 \\
\hline 50 à 100 USD & 5017 & 1822659 & 1827676 & 3123 & 581204 & 584327 \\
\hline 101 à 200 USD & 6357 & 716039 & 722396 & 4256 & 220539 & 224795 \\
\hline Plus de 200 USD & 5391 & 385161 & 390552 & 4155 & 84226 & 88381 \\
\hline Effectifs & 22176 & 6676921 & 6699097 & 13792 & 2130402 & 2144194 \\
\hline
\end{tabular}


Tableau A11 : Statut scolaire (en \%) des enfants de 5-17 ans par classe de revenu mensuel des ménages par milieu de résidence

\begin{tabular}{|c|c|c|c|c|c|c|c|}
\hline \multirow{2}{*}{$\begin{array}{l}\text { Classes de revenu } \\
\text { mensuel }\end{array}$} & \multicolumn{5}{|c|}{ Statut scolaire } & \multicolumn{2}{|c|}{ EADE } \\
\hline & Scolarisé & Déscolarisé & $\begin{array}{c}\text { Jamais } \\
\text { fréquenté }\end{array}$ & Total & Effectifs & $\%$ & Effectifs \\
\hline \multicolumn{8}{|l|}{ Urbain } \\
\hline Moins de 50 USD & 65,0 & 12,0 & 23,0 & 100,0 & 1530373 & 35,0 & 535636 \\
\hline 50-100 USD & 77,0 & 8,1 & 14,9 & 100,0 & 2357084 & 23,0 & 541833 \\
\hline 101-200 USD & 82,4 & 8,3 & 9,4 & 100,0 & 2263997 & 17,7 & 399004 \\
\hline 201-500 USD & 89,9 & 5,0 & 5,0 & 100,0 & 1787771 & 10,0 & 179936 \\
\hline Plus de 500 USD & 94,6 & 2,0 & 3,4 & 100,0 & 459862 & 5,4 & 24943 \\
\hline Total & 80,0 & 7,9 & 12,1 & 100,0 & 8399087 & 20,0 & 1681352 \\
\hline \multicolumn{8}{|l|}{ Rural } \\
\hline Moins de 50 USD & 63,1 & 7,1 & 29,8 & 100,0 & 10926848 & 36,9 & 4027778 \\
\hline 50-100 USD & 72,3 & 5,1 & 22,6 & 100,0 & 4620775 & 27,7 & 1279891 \\
\hline 101-200 USD & 74,2 & 4,2 & 21,6 & 100,0 & 1171673 & 25,8 & 302357 \\
\hline 201-500 USD & 76,3 & 5,4 & 18,3 & 100,0 & 303634 & 23,7 & 71819 \\
\hline Plus de 500 USD & 61,9 & 3,4 & 34,7 & 100,0 & 33264 & 38,1 & 12680 \\
\hline Total & 66,6 & 6,3 & 27,1 & 100,0 & 17056194 & 33,4 & 5694525 \\
\hline \multicolumn{8}{|l|}{ Ensemble } \\
\hline Moins de 50 USD & 63,4 & 7,7 & 28,9 & 100,0 & 12457221 & 36,6 & 4563414 \\
\hline 50-100 USD & 73,9 & 6,1 & 20,0 & 100,0 & 6977859 & 26,1 & 1821724 \\
\hline 101-200 USD & 79,6 & 6,9 & 13,6 & 100,0 & 3435670 & 20,5 & 701361 \\
\hline 201-500 USD & 88,0 & 5,1 & 7,0 & 100,0 & 2091405 & 12,1 & 251755 \\
\hline Plus de 500 USD & 92,4 & 2,1 & 5,5 & 100,0 & 493126 & 7,6 & 37623 \\
\hline Total & 71,0 & 6,8 & 22,1 & 100,0 & 25455281 & 28,9 & 7375877 \\
\hline
\end{tabular}

Source : Données de l'enquête auprès des ménages, EADE-RDC 2012 
Tableau A12 : Répartition (en \%) des enfants et adolescents d'âge du primaire et du secondaire en dehors de l'école selon les classes de revenu mensuel des ménages par milieu de résidence

\begin{tabular}{|c|c|c|c|c|c|c|c|}
\hline Provinces & $\begin{array}{c}\text { Moins } 50 \\
\text { dollars }\end{array}$ & $\begin{array}{l}50-100 \\
\text { dollars }\end{array}$ & $\begin{array}{c}101-200 \\
\text { dollars }\end{array}$ & $\begin{array}{c}201-500 \\
\text { dollars }\end{array}$ & $\begin{array}{c}\text { Plus de } 500 \\
\text { dollars }\end{array}$ & Total & Effectifs \\
\hline \multicolumn{8}{|l|}{ Dimension 2} \\
\hline Kinshasa & 9,4 & 35,8 & 31,1 & 20,3 & 3,4 & 100,0 & 213490 \\
\hline Bas-Congo & 47,4 & 42,8 & 8,4 & 1,4 & - & 100,0 & 157233 \\
\hline Bandundu & 77,7 & 20,3 & 1,9 & 0,1 & - & 100,0 & 274540 \\
\hline Equateur & 82,4 & 14,7 & 1,6 & 1,3 & - & 100,0 & 349836 \\
\hline Orientale & 51,4 & 29,1 & 14,3 & 4,1 & 1,1 & 100,0 & 472320 \\
\hline Nord Kivu & 75,0 & 18,2 & 4,7 & 1,8 & 0,3 & 100,0 & 457977 \\
\hline Maniema & 77,6 & 19,5 & 2,3 & 0,6 & - & 100,0 & 78884 \\
\hline sud Kivu & 58,4 & 31,5 & 9,3 & 0,7 & - & 100,0 & 235593 \\
\hline Katanga & 65,3 & 26,6 & 7,1 & 0,9 & - & 100,0 & 676760 \\
\hline Kasaï oriental & 74,4 & 17,7 & 7,6 & 0,4 & - & 100,0 & 348847 \\
\hline Kasaï occidental & 71,0 & 25,8 & 2,9 & 0,3 & - & 100,0 & 243771 \\
\hline Total & 64,3 & 24,7 & 8,1 & 2,5 & 0,4 & 100,0 & 3509252 \\
\hline \multicolumn{8}{|l|}{ Dimension 3} \\
\hline Kinshasa & 4,6 & 25,3 & 34,0 & 28,5 & 7,6 & 100,0 & 34152 \\
\hline Bas-Congo & 44,9 & 49,4 & 4,8 & 0,9 & 0,0 & 100,0 & 28816 \\
\hline Bandundu & 88,3 & 10,2 & 1,4 & 0,0 & 0,0 & 100,0 & 18490 \\
\hline Equateur & 91,2 & 8,1 & 0,7 & 0,0 & 0,0 & 100,0 & 48833 \\
\hline Orientale & 58,1 & 9,0 & 26,2 & 3,5 & 3,1 & 100,0 & 84366 \\
\hline Nord Kivu & 85,1 & 8,1 & 2,6 & 4,3 & 0,0 & 100,0 & 86286 \\
\hline Maniema & 59,0 & 29,5 & 6,0 & 5,5 & 0,0 & 100,0 & 7948 \\
\hline sud Kivu & 62,1 & 29,8 & 5,2 & 2,9 & 0,0 & 100,0 & 35770 \\
\hline Katanga & 63,3 & 23,8 & 10,6 & 2,3 & 0,0 & 100,0 & 105977 \\
\hline Kasaï oriental & 67,1 & 28,3 & 4,6 & 0,0 & 0,0 & 100,0 & 39511 \\
\hline Kasaï occidental & 63,3 & 29,8 & 3,7 & 3,2 & 0,0 & 100,0 & 23017 \\
\hline Total & 64,9 & 19,4 & 10,5 & 4,1 & 1,0 & 100,0 & 5136 \\
\hline
\end{tabular}

Source : Données de l'enquête auprès des ménages, EADE-RDC 2012

- Effectifs faibles 
Tableau A13 : Répartition (en \%) des enfants et adolescents d'âge du primaire et du secondaire en dehors de l'école selon le Statut familial de l'enfant par province

\begin{tabular}{|c|c|c|c|c|c|c|c|}
\hline Provinces & $\begin{array}{c}\text { Non } \\
\text { orphelin }\end{array}$ & $\begin{array}{l}\text { Orphelin de } \\
\text { mère }\end{array}$ & $\begin{array}{l}\text { Orphelin de } \\
\text { père }\end{array}$ & $\begin{array}{l}\text { Orphelin } \\
\text { Complet }\end{array}$ & ND & Total & Effectifs \\
\hline \multicolumn{8}{|l|}{ Dimension 2} \\
\hline Kinshasa & 77,4 & 2,6 & 17,5 & 0,7 & 1,8 & 100,0 & 213489 \\
\hline Bas-Congo & 92,5 & 0,2 & 4,1 & 1,9 & 1,3 & 100,0 & 157233 \\
\hline Bandundu & 86,7 & 2,6 & 8,0 & 1,4 & 1,4 & 100,0 & 274539 \\
\hline Equateur & 90,8 & 2,2 & 7,0 & 0,0 & - & 100,0 & 349837 \\
\hline Orientale & 82,1 & 4,3 & 7,3 & 6,3 & - & 100,0 & 472320 \\
\hline Nord Kivu & 89,9 & 1,4 & 6,6 & 1,8 & 0,2 & 100,0 & 457978 \\
\hline Maniema & 91,8 & 3,5 & 3,5 & 0,6 & 0,6 & 100,0 & 78884 \\
\hline sud Kivu & 91,2 & 1,5 & 6,1 & 1,3 & - & 100,0 & 235594 \\
\hline Katanga & 89,8 & 2,4 & 7,3 & 0,5 & - & 100,0 & 676759 \\
\hline Kasaï oriental & 89,4 & 1,1 & 8,7 & 0,7 & - & 100,0 & 348847 \\
\hline Kasaï occidental & 89,0 & 2,9 & 7,2 & 0,6 & 0,3 & 100,0 & 243772 \\
\hline Total & 88,0 & 2,3 & 7,7 & 1,6 & 0,3 & 100,0 & 3509252 \\
\hline \multicolumn{8}{|l|}{ Dimension 3} \\
\hline Kinshasa & 61,7 & 18,8 & 16,5 & 3,0 & 0,0 & 100,0 & 34152 \\
\hline Bas-Congo & 73,4 & 11,1 & 10,4 & 1,7 & 3,4 & 100,0 & 28817 \\
\hline Bandundu & 62,8 & 10,5 & 15,2 & 1,5 & 10,1 & 100,0 & 18490 \\
\hline Equateur & 80,1 & 4,6 & 15,0 & 0,3 & 0,0 & 100,0 & 48833 \\
\hline Orientale & 70,8 & 7,6 & 15,3 & 6,4 & 0,0 & 100,0 & 84366 \\
\hline Nord Kivu & 69,4 & 1,4 & 16,9 & 10,9 & 1,4 & 100,0 & 86286 \\
\hline Maniema & 70,8 & - & 11,8 & 17,5 & 0,0 & 100,0 & 7948 \\
\hline sud Kivu & 84,0 & 3,1 & 12,9 & - & 0,0 & 100,0 & 35770 \\
\hline Katanga & 76,3 & 2,4 & 18,9 & 2,4 & 0,0 & 100,0 & 105977 \\
\hline Kasaï oriental & 75,2 & 2,2 & 19,4 & 3,2 & 0,0 & 100,0 & 39511 \\
\hline Kasaï occidental & 76,3 & 3,2 & 20,5 & 0,0 & 0,0 & 100,0 & 23017 \\
\hline Total & 73,3 & 5,2 & 16,4 & 4,3 & 0,4 & 100,0 & 513167 \\
\hline
\end{tabular}


Tableau A14 : Répartition des effectifs d'enfants et d'adolescents d'âges du primaire en dehors de l'école par statut scolaire selon certaines caractéristiques

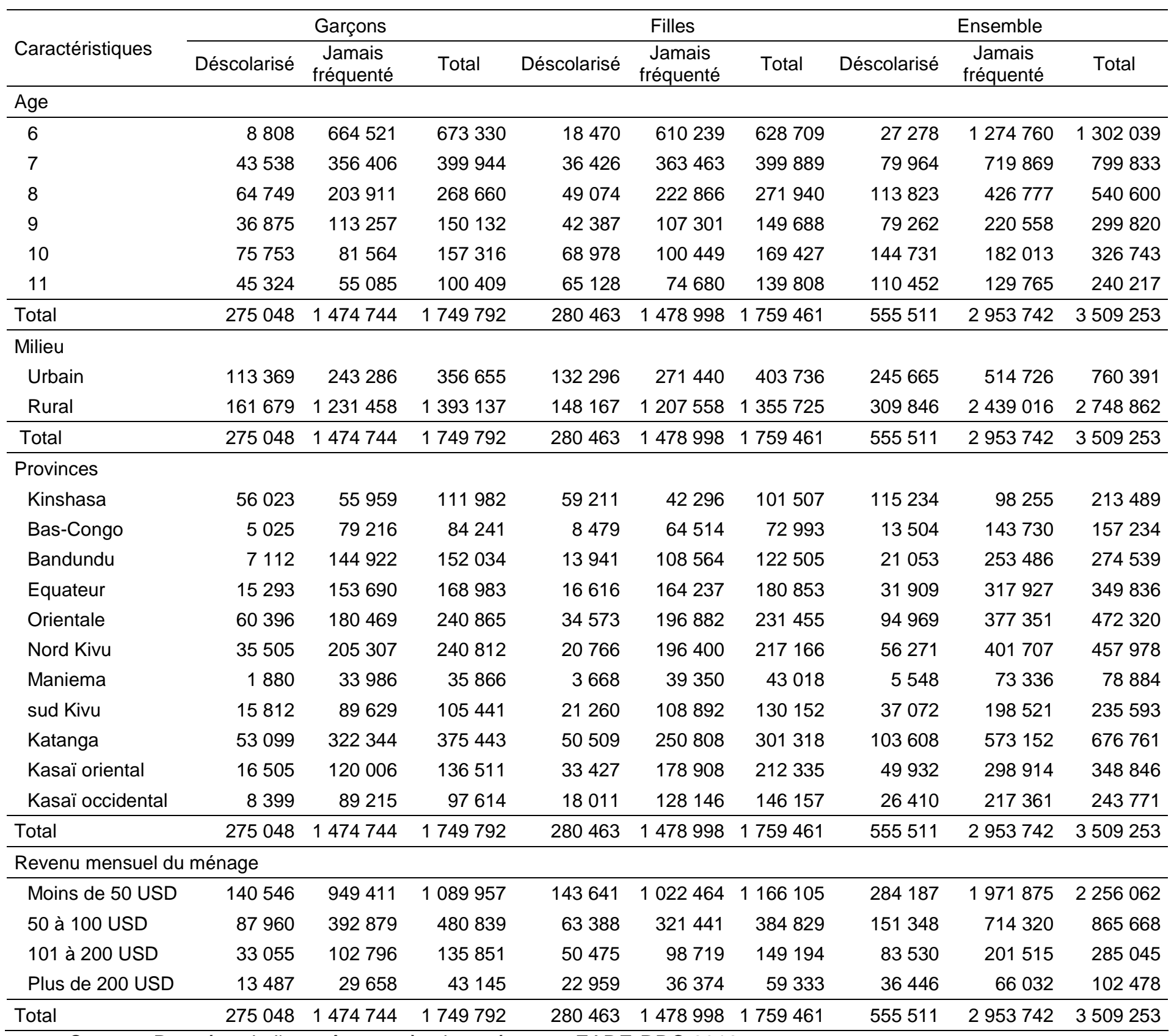

Source : Données de l'enquête auprès des ménages, EADE-RDC 2012 
Tableau A15 : Répartition des effectifs d'enfants et d'adolescents d'âges du primaire en dehors de l'école par statut scolaire selon certaines caractéristiques

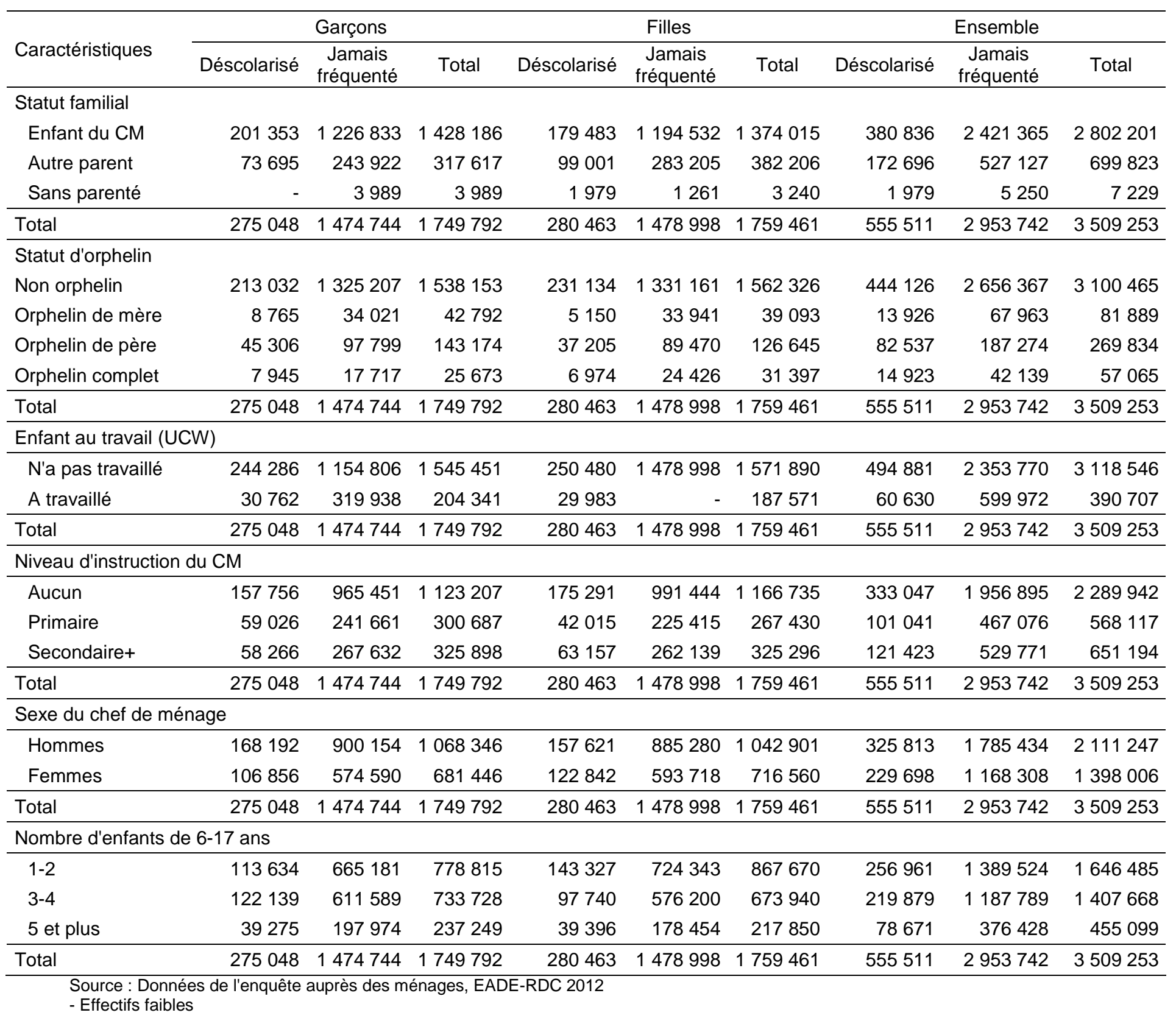


Tableau A16 : Répartition des effectifs d'enfants et d'adolescents d'âges du secondaire (12-13 ans) en dehors de l'école par statut scolaire selon certaines caractéristiques

\begin{tabular}{|c|c|c|c|c|c|c|c|c|c|}
\hline \multirow[b]{2}{*}{ Caractéristiques } & \multicolumn{3}{|c|}{ Garçons } & \multicolumn{3}{|c|}{ Filles } & \multicolumn{3}{|c|}{ Ensemble } \\
\hline & Déscolarisé & $\begin{array}{l}\text { Jamais } \\
\text { fréquenté }\end{array}$ & Total & Déscolarisé & $\begin{array}{l}\text { Jamais } \\
\text { fréquenté }\end{array}$ & Total & Déscolarisé & $\begin{array}{l}\text { Jamais } \\
\text { fréquenté }\end{array}$ & Total \\
\hline \multicolumn{10}{|l|}{ Age } \\
\hline 12 & 64499 & 51083 & 115582 & 96395 & 95535 & 191930 & 160894 & 146618 & 307512 \\
\hline 13 & 55643 & 32183 & 87826 & 59364 & 58466 & 117830 & 115007 & 90649 & 205656 \\
\hline Total & 120142 & 83266 & 203408 & 155759 & 154001 & 309760 & 275901 & 237267 & 513168 \\
\hline \multicolumn{10}{|l|}{ Milieu } \\
\hline Urbain & 40642 & 5050 & 45692 & 45145 & 28123 & 73268 & 85787 & 33173 & 118960 \\
\hline Rural & 79500 & 78216 & 157716 & 110614 & 125878 & 236492 & 190114 & 204094 & 394208 \\
\hline Total & 120142 & 83266 & 203408 & 155759 & 154001 & 309760 & 275901 & 237267 & 513168 \\
\hline \multicolumn{10}{|l|}{ Provinces } \\
\hline Kinshasa & 20055 & - & 20055 & 11838 & 2259 & 14097 & 31893 & 2259 & 34152 \\
\hline Bas-Congo & 6629 & 4060 & 10689 & 11637 & 6492 & 18129 & 18266 & 10552 & 28818 \\
\hline Bandundu & 4778 & 1949 & 6727 & 2186 & 9577 & 11763 & 6964 & 11526 & 18490 \\
\hline Equateur & 5981 & 7875 & 13856 & 11923 & 23054 & 34977 & 17904 & 30929 & 48833 \\
\hline Orientale & 25021 & 16302 & 41323 & 25425 & 17618 & 43043 & 50446 & 33920 & 84366 \\
\hline Nord Kivu & 14052 & 12593 & 26645 & 27695 & 31947 & 59642 & 41747 & 44541 & 86287 \\
\hline Maniema & 2381 & 2381 & 4762 & 917 & 2268 & 3185 & 3298 & 4649 & 7947 \\
\hline sud Kivu & 9425 & 8641 & 18066 & 9898 & 7806 & 17704 & 19323 & 16447 & 35770 \\
\hline Katanga & 22424 & 24107 & 46530 & 27522 & 31925 & 59447 & 49946 & 56032 & 105977 \\
\hline Kasaï oriental & 5267 & 2285 & 7552 & 14589 & 17369 & 31958 & 19856 & 19654 & 39510 \\
\hline Kasaï occidental & 4130 & 3072 & 7202 & 12129 & 3685 & 15814 & 16259 & 6757 & 23016 \\
\hline Total & 120142 & 83266 & 203408 & 155759 & 154001 & 309760 & 275901 & 237267 & 513168 \\
\hline \multicolumn{10}{|c|}{ Revenu mensuel du ménage } \\
\hline Moins de 50 USD & 66809 & 63031 & 129840 & 92928 & 110168 & 203096 & 159737 & 173198 & 332935 \\
\hline 50 à 100 USD & 23679 & 14461 & 38140 & 36197 & 25230 & 61427 & 59876 & 39691 & 99567 \\
\hline 101 à 200 USD & 22190 & 254 & 22444 & 19265 & 12428 & 31693 & 41455 & 12682 & 54137 \\
\hline Plus de 200 USD & 7464 & 5520 & 12984 & 7369 & 6175 & 13544 & 14833 & 11695 & 26528 \\
\hline Total & 120142 & 83266 & 203408 & 155759 & 154001 & 309760 & 275901 & 237267 & 513168 \\
\hline
\end{tabular}


Tableau A17 : Répartition des effectifs d'enfants et d'adolescents d'âges du secondaire (12-13 ans) en dehors de l'école par statut scolaire selon certaines caractéristiques

\begin{tabular}{|c|c|c|c|c|c|c|c|c|c|}
\hline \multirow[b]{2}{*}{ Caractéristiques } & \multicolumn{3}{|c|}{ Garçons } & \multicolumn{3}{|c|}{ Filles } & \multicolumn{3}{|c|}{ Ensemble } \\
\hline & Déscolarisé & $\begin{array}{l}\text { Jamais } \\
\text { fréquenté }\end{array}$ & Total & Déscolarisé & $\begin{array}{l}\text { Jamais } \\
\text { fréquenté }\end{array}$ & Total & Déscolarisé & $\begin{array}{c}\text { Jamais } \\
\text { fréquenté }\end{array}$ & Total \\
\hline \multicolumn{10}{|l|}{ Statut familial } \\
\hline Enfant du CM & 84336 & 54215 & 138551 & 103919 & 104112 & 208031 & 188255 & 158327 & 346582 \\
\hline Autre parent & 35723 & 29051 & 64774 & 50866 & 49889 & 100755 & 86589 & 78940 & 165529 \\
\hline Sans parenté & 83 & - & 83 & 974 & - & 974 & 1057 & - & 1057 \\
\hline Total & 120142 & 83266 & 203408 & 155759 & 154001 & 309760 & 275901 & 237267 & 513168 \\
\hline \multicolumn{10}{|l|}{ statut d'orphelin } \\
\hline Non orphelin & 80944 & 63163 & 144149 & 115196 & 120069 & 235246 & 196157 & 183218 & 379375 \\
\hline Orphelin de mère & 5692 & 3657 & 9348 & 11587 & 5903 & 17506 & 17286 & 9564 & 26850 \\
\hline Orphelin de père & 29519 & 8972 & 38422 & 23910 & 22486 & 46399 & 53405 & 31433 & 84837 \\
\hline Orphelin complet & 3987 & 7474 & 11489 & 5067 & 5543 & 10608 & 9054 & 13052 & 22106 \\
\hline Total & 120142 & 83266 & 203408 & 155759 & 154001 & 309760 & 275901 & 237267 & 513168 \\
\hline \multicolumn{10}{|c|}{ Enfant au travail (UCW) } \\
\hline N'a pas travaillé & 90381 & 83266 & 155243 & 121330 & 104452 & 240558 & 211201 & 211425 & 395020 \\
\hline A travaillé & 29761 & 0 & 48165 & 34429 & 49549 & 69202 & 64700 & 25842 & 118148 \\
\hline Total & 120142 & 83266 & 203408 & 155759 & 154001 & 309760 & 275901 & 237267 & 513168 \\
\hline \multicolumn{10}{|c|}{ Niveau d'instruction du CM } \\
\hline Aucun & 48858 & 42493 & 91351 & 71694 & 75772 & 147466 & 120552 & 118265 & 238817 \\
\hline Primaire & 35766 & 21377 & 57143 & 36630 & 42680 & 79310 & 72396 & 64057 & 136453 \\
\hline Secondaire+ & 35519 & 19396 & 54915 & 47434 & 35549 & 82983 & 82953 & 54945 & 137898 \\
\hline Total & 120142 & 83266 & 203408 & 155759 & 154001 & 309760 & 275901 & 237267 & 513168 \\
\hline \multicolumn{10}{|c|}{ Sexe du chef de ménage } \\
\hline Hommes & 86941 & 57913 & 144854 & 92667 & 106757 & 199424 & 179608 & 164670 & 344278 \\
\hline Femmes & 33201 & 25353 & 58554 & 63092 & 47244 & 110336 & 96293 & 72597 & 168890 \\
\hline Total & 120142 & 83266 & 203408 & 155759 & 154001 & 309760 & 275901 & 237267 & 513168 \\
\hline \multicolumn{10}{|c|}{ Nombre d'enfants de 6-17 ans } \\
\hline $1-2$ & 44810 & 29533 & 74343 & 74359 & 75138 & 149497 & 119169 & 104671 & 223840 \\
\hline $3-4$ & 53643 & 29603 & 83246 & 55429 & 52965 & 108394 & 109072 & 82568 & 191640 \\
\hline 5 et plus & 21689 & 24130 & 45819 & 25971 & 25898 & 51869 & 47660 & 50028 & 97688 \\
\hline Total & 120142 & 83266 & 203408 & 155759 & 154001 & 309760 & 275901 & 237267 & 513168 \\
\hline
\end{tabular}

Source : Données de l'enquête auprès des ménages, EADE-RDC 2012

- Effectifs faibles 
Tableau A18 : Probabilités cumulées (en \%) d'abandonner par sexe et par milieu de résidence

\begin{tabular}{cccrrc}
\hline & \multicolumn{2}{c}{ Sexe } & \multicolumn{3}{c}{ Milieu } \\
\cline { 2 - 6 } Année & Garçons & \multicolumn{1}{c}{ Filles } & Urbain & Rural & Ensemble \\
\hline 1 & 2,3 & 3,1 & 2,3 & 3,4 & 2,7 \\
2 & 4,2 & 5,4 & 4,4 & 5,9 & 4,8 \\
3 & 6,2 & 8,3 & 6,6 & 8,8 & 7,2 \\
4 & 8,1 & 11,0 & 8,7 & 11,4 & 9,5 \\
5 & 10,2 & 13,5 & 10,9 & 14,3 & 11,8 \\
6 & 12,1 & 16,6 & 13,1 & 17,1 & 14,3 \\
7 & 14,9 & 21,0 & 16,4 & 20,9 & 17,8 \\
8 & 19,4 & 25,7 & 19,3 & 25,9 & 22,4 \\
9 & 21,9 & 29,7 & 21,3 & 31,3 & 25,7 \\
10 & 24,1 & 32,5 & 22,9 & 34,6 & 28,1 \\
11 & 26,8 & 35,7 & 25,0 & 38,1 & 31,1 \\
12 & 30,7 & 38,8 & 27,4 & 45,6 & 34,7 \\
13 & 30,7 & 39,4 & 27,9 & 45,6 & 34,7 \\
14 & 30,7 & 39,4 & 27,9 & 45,6 & 34,7 \\
15 & 30,7 & 39,4 & 27,9 & 45,6 & 34,7 \\
16 & 30,7 & 39,4 & 27,9 & 45,6 & 34,7 \\
17 & 30,7 & 39,4 & 27,9 & 45,6 & 34,7 \\
\hline Effectif enfants & 10696 & 10079 & 10313 & 10462 & 20775 \\
\hline Source : Données de l'enquête auprès des ménages, EADE-RDC 2012
\end{tabular}

Tableau A19 : Probabilités cumulées (en \%) d'abandonner par province

\begin{tabular}{|c|c|c|c|c|c|c|c|c|c|c|c|}
\hline $\begin{array}{c}\text { Age } \\
\text { d'entrée }\end{array}$ & Kinshasa & $\begin{array}{c}\text { Bas- } \\
\text { Congo }\end{array}$ & Bandundu & Equateur & Orientale & Nord Kivu & Maniema & Sud Kivu & Katanga & $\begin{array}{l}\text { Kasaï } \\
\text { Orient. }\end{array}$ & $\begin{array}{c}\text { Kasaï } \\
\text { Occident. }\end{array}$ \\
\hline 1 & 2,1 & 1,7 & 0,6 & 2,1 & 4,2 & 3,6 & 1,7 & 3,6 & 2,6 & 3,9 & 3,4 \\
\hline 2 & 4,0 & 2,9 & 1,7 & 4,1 & 6,3 & 6,9 & 3,0 & 5,7 & 5,5 & 5,9 & 5,7 \\
\hline 3 & 5,6 & 4,8 & 2,1 & 7,4 & 8,6 & 10,5 & 4,3 & 7,6 & 10,0 & 8,6 & 7,8 \\
\hline 4 & 8,3 & 6,1 & 2,6 & 8,5 & 13,1 & 13,2 & 6,1 & 10,0 & 12,6 & 11,1 & 8,8 \\
\hline 5 & 10,3 & 7,8 & 3,7 & 9,3 & 15,8 & 16,3 & 7,2 & 11,8 & 16,0 & 14,5 & 10,9 \\
\hline 6 & 12,3 & 9,8 & 4,3 & 10,2 & 19,2 & 21,8 & 8,8 & 13,7 & 18,1 & 18,7 & 13,4 \\
\hline 7 & 14,4 & 14,4 & 5,0 & 13,0 & 24,8 & 26,6 & 12,5 & 19,1 & 21,0 & 23,6 & 16,6 \\
\hline 8 & 19,4 & 16,3 & 5,8 & 14,9 & 35,9 & 31,0 & 12,8 & 22,7 & 26,3 & 27,1 & 20,4 \\
\hline 9 & 20,3 & 19,3 & 6,3 & 19,9 & 35,9 & 36,0 & 14,6 & 29,7 & 30,1 & 33,6 & 20,4 \\
\hline 10 & 21,4 & 22,6 & 6,3 & 26,5 & 35,9 & 39,8 & 14,6 & 34,1 & 31,7 & 35,2 & 26,4 \\
\hline 11 & 24,3 & 31,3 & 6,3 & 26,5 & 35,9 & 39,8 & 14,6 & 36,9 & 33,4 & 35,5 & 38,5 \\
\hline 12 & 26,7 & 31,3 & 6,3 & 26,5 & 35,9 & 39,8 & 14,6 & 36,9 & 34,0 & 35,5 & 38,5 \\
\hline 13 & 26,7 & 31,3 & 6,3 & 26,5 & 35,9 & 39,8 & 14,6 & 36,9 & 34,0 & 35,5 & 38,5 \\
\hline 14 & 26,7 & 31,3 & 6,3 & 26,5 & 35,9 & 39,8 & 14,6 & 36,9 & 34,0 & 35,5 & 38,5 \\
\hline 15 & 26,7 & 31,3 & 6,3 & 26,5 & 35,9 & 39,8 & 14,6 & 36,9 & 34,0 & 35,5 & 38,5 \\
\hline 16 & 26,7 & 31,3 & 6,3 & 26,5 & 35,9 & 39,8 & 14,6 & 36,9 & 34,0 & 35,5 & 38,5 \\
\hline 17 & 26,7 & 31,3 & 6,3 & 26,5 & 35,9 & 39,8 & 14,6 & 36,9 & 34,0 & 35,5 & 38,5 \\
\hline $\begin{array}{l}\text { Effectif } \\
\text { enfants }\end{array}$ & 2001 & 1859 & 1776 & 1346 & 1681 & 2197 & 1219 & 1957 & 2730 & 1842 & 2167 \\
\hline
\end{tabular}


Tableau A20 : Probabilités cumulées (en \%) d'entrée à l'école par sexe et par milieu

\begin{tabular}{|c|c|c|c|c|c|}
\hline \multirow[b]{2}{*}{ Age d'entrée } & \multicolumn{2}{|c|}{ Sexe } & \multicolumn{2}{|c|}{ Milieu de résidence } & \multirow[b]{2}{*}{ Ensemble } \\
\hline & Garçons & Filles & Urbain & Rural & \\
\hline 3 & 0,9 & 0,7 & 1,8 & 0,3 & 0,8 \\
\hline 4 & 2,3 & 2,4 & 5,6 & 0,7 & 2,3 \\
\hline 5 & 6,5 & 6,6 & 13,5 & 3,0 & 6,5 \\
\hline 6 & 20,9 & 20,3 & 34,5 & 13,5 & 20,6 \\
\hline 7 & 42,9 & 39,4 & 58,3 & 32,3 & 41,1 \\
\hline 8 & 60,5 & 57,2 & 72,6 & 51,8 & 58,9 \\
\hline 9 & 73,1 & 69,8 & 79,1 & 67,6 & 71,5 \\
\hline 10 & 80,9 & 78,0 & 82,9 & 77,8 & 79,5 \\
\hline 11 & 86,5 & 83,4 & 85,9 & 84,7 & 85,0 \\
\hline & & 11 & & & 23 \\
\hline Effectif enfant & 12072 & 647 & 10975 & 12744 & 719 \\
\hline
\end{tabular}

Tableau A21 : Probabilités cumulées (en \%) d'entrée à l'école par province

\begin{tabular}{|c|c|c|c|c|c|c|c|c|c|c|c|}
\hline $\begin{array}{c}\text { Age } \\
\text { d'entrée }\end{array}$ & Kinshasa & $\begin{array}{c}\text { Bas- } \\
\text { Congo }\end{array}$ & Bandundu & Equateur & Orientale & Nord Kivu & Maniema & Sud Kivu & Katanga & $\begin{array}{l}\text { Kasaï } \\
\text { Orient. }\end{array}$ & $\begin{array}{c}\text { Kasaï } \\
\text { Occident. }\end{array}$ \\
\hline 3 & 3,0 & 0,6 & 0,5 & 0,6 & 0,5 & 0,2 & 0,2 & 0,2 & 0,9 & 0,2 & 0,1 \\
\hline 4 & 8,7 & 1,4 & 1,2 & 2,0 & 1,5 & 0,7 & 0,8 & 1,1 & 2,5 & 0,8 & 0,2 \\
\hline 5 & 20,2 & 4,0 & 3,8 & 4,2 & 6,7 & 2,4 & 4,0 & 4,3 & 6,1 & 3,8 & 3,0 \\
\hline 6 & 44,8 & 15,3 & 15,8 & 15,6 & 18,8 & 13,4 & 16,4 & 19,1 & 20,6 & 17,8 & 12,6 \\
\hline 7 & 65,0 & 40,5 & 35,6 & 35,4 & 37,9 & 33,2 & 37,0 & 41,2 & 42,3 & 36,2 & 33,4 \\
\hline 8 & 73,9 & 63,3 & 53,3 & 57,1 & 57,6 & 52,5 & 57,1 & 61,6 & 56,9 & 54,5 & 56,2 \\
\hline 9 & 78,1 & 78,0 & 70,4 & 71,4 & 71,5 & 65,6 & 73,4 & 73,2 & 68,6 & 67,5 & 71,3 \\
\hline 10 & 80,7 & 87,3 & 80,4 & 80,2 & 78,5 & 74,0 & 80,3 & 83,2 & 77,4 & 77,0 & 81,3 \\
\hline 11 & 83,1 & 91,8 & 87,0 & 88,2 & 83,5 & 79,1 & 87,5 & 90,3 & 83,2 & 82,4 & 86,9 \\
\hline $\begin{array}{l}\text { Effectif } \\
\text { enfants }\end{array}$ & 2066 & 2031 & 1950 & 1551 & 1888 & 2728 & 1391 & 2197 & 3300 & 2086 & 2531 \\
\hline
\end{tabular}


Tableau A22 : Répartition (en \%) du statut scolaire des enfants et adolescents scolarisés au primaire selon le retard scolaire par classe du primaire par sexe et par milieu de résidence

\begin{tabular}{|c|c|c|c|c|c|c|c|}
\hline \multirow{2}{*}{$\begin{array}{l}\text { Situation } \\
\text { scolaire }\end{array}$} & \multicolumn{6}{|c|}{ Primaire } & \multirow{2}{*}{ Ensemble } \\
\hline & 1ère année & 2ème année & 3ème année & 4ème année & 5ème année & 6ème année & \\
\hline \multicolumn{8}{|l|}{ Ensemble } \\
\hline Précoce & 12,5 & 7,0 & 4,4 & 3,4 & 2,7 & 3,2 & 6,3 \\
\hline Normal & 39,1 & 18,4 & 14,8 & 11,3 & 10,6 & 9,3 & 16,5 \\
\hline Tardif 1 an & 60,9 & 74,6 & 80,8 & 85,3 & 86,7 & 87,6 & 77,2 \\
\hline Tardif 2 ans & 33,9 & 50,7 & 60,7 & 66,5 & 71,2 & 65,6 & 55,3 \\
\hline Tardif 3 ans + & 18,1 & 32,7 & 41,9 & 49,0 & 50,2 & 50,0 & 37,7 \\
\hline Total & 100,0 & 100,0 & 100,0 & 100,0 & 100,0 & 100,0 & 100,0 \\
\hline Effectifs & 3447296 & 3098415 & 2663498 & 2302475 & 1887239 & 1823105 & 15226690 \\
\hline \multicolumn{8}{|l|}{ Garçons } \\
\hline Précoce & 12,1 & 8,0 & 4,1 & 3,7 & 2,0 & 3,0 & 6,2 \\
\hline Normal & 27,1 & 18,8 & 15,6 & 11,4 & 10,6 & 9,0 & 16,7 \\
\hline Tardif 1 an & 60,8 & 73,2 & 80,3 & 85,0 & 87,4 & 88,0 & 77,2 \\
\hline Tardif 2 ans & 31,6 & 50,8 & 58,9 & 66,4 & 71,9 & 65,2 & 54,8 \\
\hline Tardif 3 ans + & 17,2 & 32,6 & 40,2 & 50,2 & 51,2 & 49,8 & 37,7 \\
\hline Total & 100,0 & 100,0 & 100,0 & 100,0 & 100,0 & 100,0 & 100,0 \\
\hline Effectifs & 1849581 & 1587878 & 1364137 & 1233402 & 1041174 & 1049689 & 8126073 \\
\hline \multicolumn{8}{|l|}{ Filles } \\
\hline Précoce & 12,9 & 6,0 & 4,8 & 3,1 & 3,7 & 3,5 & 6,4 \\
\hline Normal & 26,1 & 18,0 & 13,8 & 11,2 & 10,5 & 9,8 & 16,2 \\
\hline Tardif 1 an & 61,0 & 76,0 & 81,4 & 85,6 & 85,8 & 86,7 & 77,4 \\
\hline Tardif 2 ans & 36,5 & 50,6 & 62,6 & 66,5 & 70,2 & 65,9 & 56,0 \\
\hline Tardif 3 ans + & 19,1 & 32,8 & 43,7 & 47,6 & 48,9 & 50,2 & 37,8 \\
\hline Total & 100,0 & 100,0 & 100,0 & 100,0 & 100,0 & 100,0 & 100,0 \\
\hline Effectifs & 1597715 & 1510537 & 1299361 & 1069073 & 846065 & 773416 & 7100617 \\
\hline \multicolumn{8}{|l|}{ Urbain } \\
\hline Précoce & 19,2 & 12,5 & 10,2 & 6,9 & 5,5 & 5,3 & 10,5 \\
\hline Normal & 37,5 & 28,6 & 28,0 & 20,9 & 20,9 & 17,4 & 26,3 \\
\hline Tardif 1 an & 43,3 & 58,9 & 61,9 & 72,2 & 73,6 & 77,3 & 63,3 \\
\hline Tardif 2 ans & 17,3 & 31,0 & 37,3 & 45,0 & 49,6 & 49,5 & 37,0 \\
\hline Tardif 3 ans + & 6,7 & 16,2 & 23,0 & 28,6 & 29,0 & 34,4 & 22,0 \\
\hline Total & 100,0 & 100,0 & 100,0 & 100,0 & 100,0 & 100,0 & 100,0 \\
\hline Effectifs & 946613 & 865316 & 843632 & 788642 & 644236 & 704398 & 4794165 \\
\hline \multicolumn{8}{|l|}{ Rural } \\
\hline Précoce & 9,9 & 4,9 & 1,7 & 1,6 & 1,3 & 1,9 & 4,3 \\
\hline Normal & 22,5 & 14,5 & 8,6 & 6,3 & 5,2 & 4,2 & 12,0 \\
\hline Tardif 1 an & 67,6 & 80,6 & 89,6 & 92,1 & 93,6 & 93,9 & 83,7 \\
\hline Tardif 2 ans & 40,2 & 58,3 & 71,5 & 77,6 & 82,4 & 75,6 & 63,8 \\
\hline Tardif 3 ans + & 22,4 & 39,1 & 50,7 & 59,6 & 61,2 & 59,8 & 45,0 \\
\hline Total & 100,0 & 100,0 & 100,0 & 100,0 & 100,0 & 100,0 & 100,0 \\
\hline Effectifs & 2500682 & 2233098 & 1819867 & 1513832 & 1243002 & 1118709 & 10432523 \\
\hline
\end{tabular}


Tableau A23 : Proportion (en \%) des enfants et adolescents scolarisés au secondaire $1^{\text {er }}$ cycle selon le retard scolaire par classe du secondaire par sexe et par milieu de résidence

\begin{tabular}{|c|c|c|c|}
\hline \multirow{2}{*}{ Situation scolaire } & \multicolumn{2}{|c|}{ Secondaire } & \multirow{2}{*}{ Ensemble } \\
\hline & 1ère année & 2ème année & \\
\hline \multicolumn{4}{|l|}{ Ensemble } \\
\hline Précoce & 2,6 & 4,3 & 3,8 \\
\hline Normal & 8,6 & 9,1 & 9,5 \\
\hline Tardif 1 an & 88,9 & 86,6 & 86,7 \\
\hline Tardif 2 ans & 75,3 & 68,7 & 70,4 \\
\hline Tardif 3 ans + & 57,5 & 48,3 & 52,8 \\
\hline Total & 100,0 & 100,0 & 100,0 \\
\hline Effectifs & 1247241 & 1074834 & 2322075 \\
\hline \multicolumn{4}{|l|}{ Garçons } \\
\hline Précoce & 2,1 & 5,6 & 4,0 \\
\hline Normal & 8,5 & 7,3 & 8,8 \\
\hline Tardif 1 an & 89,4 & 87,1 & 87,2 \\
\hline Tardif 2 ans & 74,8 & 71,2 & 71,8 \\
\hline Tardif 3 ans + & 56,2 & 51,3 & 54,2 \\
\hline Total & 100,0 & 100,0 & 100,0 \\
\hline Effectifs & 710142 & 626125 & 1336267 \\
\hline \multicolumn{4}{|l|}{ Filles } \\
\hline Précoce & 3,2 & 2,5 & 3,5 \\
\hline Normal & 8,6 & 11,6 & 10,5 \\
\hline Tardif 1 an & 88,3 & 86,0 & 85,9 \\
\hline Tardif 2 ans & 75,9 & 65,4 & 68,3 \\
\hline Tardif 3 ans + & 59,1 & 44,2 & 50,7 \\
\hline Total & 100,0 & 100,0 & 100,0 \\
\hline Effectifs & 537099 & 448709 & 985808 \\
\hline \multicolumn{4}{|l|}{ Urbain } \\
\hline Précoce & 3,9 & 7,3 & 5,6 \\
\hline Normal & 17,2 & 15,3 & 15,8 \\
\hline Tardif 1 an & 78,9 & 77,3 & 78,7 \\
\hline Tardif 2 ans & 61,3 & 54,8 & 57,1 \\
\hline Tardif 3 ans + & 43,1 & 32,2 & 37,5 \\
\hline Total & 100,0 & 100,0 & 100,0 \\
\hline Effectifs & 521814 & 521548 & 1043362 \\
\hline \multicolumn{4}{|l|}{ Rural } \\
\hline Précoce & 1,7 & 1,5 & 2,2 \\
\hline Normal & 2,3 & 3,3 & 3,8 \\
\hline Tardif 1 an & 96,0 & 95,1 & 93,9 \\
\hline Tardif 2 ans & 85,3 & 81,7 & 82,4 \\
\hline Tardif 3 ans + & 67,8 & 63,4 & 66,6 \\
\hline Total & 100,0 & 100,0 & 100,0 \\
\hline Effectifs & 725429 & 553290 & 1278719 \\
\hline
\end{tabular}

Source : Données de l'enquête auprès des ménages, EADE-RDC 2012 
Tableau A24 : Répartition (en \%) du statut scolaire des enfants et adolescents scolarisés au primaire par province et par sexe

\begin{tabular}{|c|c|c|c|c|c|c|c|}
\hline Provinces & Précoce & Normal & Tardif 1 an & Tardif 2 ans & Tardif 3 ans + & Total & Effectifs \\
\hline \multicolumn{8}{|l|}{ Garçons } \\
\hline Kinshasa & 12,7 & 33,4 & 25,2 & 15,3 & 13,4 & 100,0 & 1213918 \\
\hline Bas-Congo & 3,6 & 9,0 & 17,2 & 19,7 & 50,5 & 100,0 & 639126 \\
\hline Bandundu & 5,4 & 10,7 & 19,7 & 17,2 & 47,0 & 100,0 & 1278652 \\
\hline Equateur & 5,0 & 11,9 & 18,6 & 18,1 & 46,4 & 100,0 & 1107315 \\
\hline Orientale & 5,0 & 12,2 & 20,9 & 18,3 & 43,5 & 100,0 & 1244285 \\
\hline Nord Kivu & 1,5 & 12,3 & 24,7 & 18,1 & 43,3 & 100,0 & 667494 \\
\hline Maniema & 6,2 & 14,5 & 20,4 & 17,2 & 41,7 & 100,0 & 300942 \\
\hline sud Kivu & 5,4 & 17,6 & 21,5 & 16,3 & 39,3 & 100,0 & 640219 \\
\hline Katanga & 6,1 & 15,8 & 20,4 & 14,9 & 42,8 & 100,0 & 1481994 \\
\hline Kasaï oriental & 5,0 & 11,2 & 22,6 & 18,4 & 42,8 & 100,0 & 881235 \\
\hline Kasaï occidental & 3,6 & 12,3 & 19,2 & 17,7 & 47,1 & 100,0 & 580590 \\
\hline Total & 5,8 & 15,2 & 21,0 & 17,2 & 40,8 & 100,0 & 10035770 \\
\hline \multicolumn{8}{|l|}{ Filles } \\
\hline Kinshasa & 14,3 & 31,4 & 26,9 & 12,9 & 14,4 & 100,0 & 1186883 \\
\hline Bas-Congo & 3,3 & 11,7 & 15,3 & 21,8 & 48,0 & 100,0 & 451929 \\
\hline Bandundu & 5,2 & 12,4 & 18,8 & 18,4 & 45,1 & 100,0 & 1102549 \\
\hline Equateur & 3,0 & 13,3 & 19,0 & 15,9 & 48,8 & 100,0 & 950730 \\
\hline Orientale & 4,9 & 11,7 & 18,8 & 20,4 & 44,3 & 100,0 & 1021243 \\
\hline Nord Kivu & 2,5 & 9,2 & 24,3 & 22,9 & 41,1 & 100,0 & 596655 \\
\hline Maniema & 5,0 & 14,3 & 20,4 & 18,3 & 42,0 & 100,0 & 255896 \\
\hline sud Kivu & 2,7 & 12,0 & 23,9 & 20,8 & 40,5 & 100,0 & 537272 \\
\hline Katanga & 6,1 & 16,2 & 21,5 & 17,2 & 39,0 & 100,0 & 1144827 \\
\hline Kasaï oriental & 6,3 & 13,8 & 16,9 & 18,8 & 44,3 & 100,0 & 705798 \\
\hline Kasaï occidental & 4,7 & 9,6 & 19,5 & 19,0 & 47,3 & 100,0 & 454653 \\
\hline Total & 5,9 & 15,3 & 20,8 & 18,1 & 39,8 & 100,0 & 8408435 \\
\hline \multicolumn{8}{|l|}{$\mathrm{G}+\mathrm{F}$} \\
\hline Kinshasa & 13,5 & 32,4 & 26,1 & 14,1 & 13,9 & 100,0 & 2400801 \\
\hline Bas-Congo & 3,5 & 10,1 & 16,4 & 20,6 & 49,5 & 100,0 & 1091055 \\
\hline Bandundu & 5,3 & 11,5 & 19,3 & 17,8 & 46,1 & 100,0 & 2381201 \\
\hline Equateur & 4,0 & 12,5 & 18,8 & 17,1 & 47,5 & 100,0 & 2058045 \\
\hline Orientale & 5,0 & 12,0 & 20,0 & 19,2 & 43,9 & 100,0 & 2265528 \\
\hline Nord Kivu & 2,0 & 10,8 & 24,5 & 20,4 & 42,2 & 100,0 & 1264149 \\
\hline Maniema & 5,7 & 14,4 & 20,4 & 17,7 & 41,9 & 100,0 & 556838 \\
\hline sud Kivu & 4,2 & 15,0 & 22,6 & 18,4 & 39,8 & 100,0 & 1177491 \\
\hline Katanga & 6,1 & 16,0 & 20,9 & 15,9 & 41,1 & 100,0 & 2626821 \\
\hline Kasaï oriental & 5,6 & 12,3 & 20,1 & 18,6 & 43,5 & 100,0 & 1587033 \\
\hline Kasaï occidental & 4,1 & 11,1 & 19,3 & 18,3 & 47,2 & 100,0 & 1035243 \\
\hline Ensemble RDC & 5,9 & 15,3 & 20,9 & 17,6 & 40,3 & 100,0 & 18444205 \\
\hline
\end{tabular}

Source : Données de l'enquête auprès des ménages, EADE-RDC 2012 
Tableau A25 : Répartition (en \%) de la population de 6-11 ans selon le statut d'exclusion par caractéristique

\begin{tabular}{|c|c|c|c|c|c|c|c|c|c|c|c|c|}
\hline \multirow{2}{*}{ Caractéristiques } & \multicolumn{4}{|c|}{ Urbain } & \multicolumn{4}{|c|}{ Rural } & \multicolumn{4}{|c|}{ Ensemble } \\
\hline & Non & Oui & Total & Total & Non & Oui & Total & Total & Non & Oui & Total & Total \\
\hline \multicolumn{13}{|c|}{ Sexe du membre du ménage } \\
\hline Garçons & 83,1 & 16,9 & 100,0 & 2107946 & 70,3 & 29,7 & 100,0 & 4683559 & 74,2 & 25,8 & 100,0 & 6791505 \\
\hline Filles & 80,7 & 19,3 & 100,0 & 2094104 & 68,0 & 32,0 & 100,0 & 4231987 & 72,2 & 27,8 & 100,0 & 6326091 \\
\hline V de Cramer & \multicolumn{4}{|c|}{0,013} & \multicolumn{4}{|c|}{0,034} & \multicolumn{4}{|c|}{0,023} \\
\hline Seuil de significativité & & & & & & & & & & & & \\
\hline Revenu mensuel du mér & & & & & & & & & & & & \\
\hline Moins de 50 \$US & 64,6 & 35,4 & 100,0 & 821370 & 66,0 & 34,0 & 100,0 & 5782299 & 65,8 & 34,2 & 100,0 & 6603670 \\
\hline 50 à 100 \$US & 79,7 & 20,3 & 100,0 & 1223059 & 74,3 & 25,7 & 100,0 & 2397934 & 76,1 & 23,9 & 100,0 & 3620993 \\
\hline 101 à 200 \$US & 85,9 & 14,1 & 100,0 & 1097282 & 77,9 & 22,1 & 100,0 & 587285 & 83,1 & 16,9 & 100,0 & 1684567 \\
\hline Plus de 200 \$US & 93,8 & 6,2 & 100,0 & 1060338 & 75,5 & 24,5 & 100,0 & 148028 & 91,5 & 8,5 & 100,0 & 1208366 \\
\hline D de Somers & & & & & & & & & & & & \\
\hline Seuil de significativité & & & & & & & & & & & & \\
\hline Statut familial & & & & & & & & & & & & \\
\hline Enfant du CM & 83,4 & 16,6 & 100,0 & 3095449 & 69,7 & 30,3 & 100,0 & 7549411 & 73,7 & 26,3 & 100,0 & 10644860 \\
\hline Autre parent & 77,6 & 22,4 & 100,0 & 1103292 & 66,6 & 33,4 & 100,0 & 1355250 & 71,5 & 28,5 & 100,0 & 2458542 \\
\hline Sans parenté & $100,0-$ & & 100,0 & 3308 & 33,6 & 66,4 & 100,0 & 10886 & 49,1 & 50,9 & 100,0 & 14194 \\
\hline D de Somers & & & & & & & & & & & & \\
\hline Seuil de significativité & & & & & & & & & & & & \\
\hline Statut d'orphelin & & & & & & & & & & & & \\
\hline Non orphelin & 82,4 & 17,6 & 100,0 & 3733654 & 69,9 & 30,1 & 100,0 & 8100902 & 73,8 & 26,2 & 100,0 & 11834556 \\
\hline Orphelin de mère & 76,1 & 23,9 & 100,0 & 98490 & 69,3 & 30,7 & 100,0 & 189640 & 71,6 & 28,4 & 100,0 & 288131 \\
\hline Orphelin de père & 77,6 & 22,4 & 100,0 & 301044 & 61,0 & 39,0 & 100,0 & 518107 & 67,1 & 32,9 & 100,0 & 819151 \\
\hline Orphelin complet & 83,8 & 16,2 & 100,0 & 66353 & 57,7 & 42,3 & 100,0 & 109405 & 67,6 & 32,4 & 100,0 & 175758 \\
\hline V de Cramer & & & & & & & & & & & & \\
\hline Seuil de significativité & & & & & & & & & & & & \\
\hline Niveau d'instruction du & & & & & & & & & & & & \\
\hline Aucun & 79,3 & 20,7 & 100,0 & 2371778 & 66,7 & 33,3 & 100,0 & 5408402 & 70,6 & 29,4 & 100,0 & 7780181 \\
\hline Primaire & 69,5 & 30,5 & 100,0 & 148083 & 61,4 & 38,6 & 100,0 & 1353065 & 62,2 & 37,8 & 100,0 & 1501148 \\
\hline Secondaire+ & 86,7 & 13,3 & 100,0 & 1682188 & 80,2 & 19,8 & 100,0 & 2154079 & 83,0 & 17,0 & 100,0 & 3836267 \\
\hline D de Somers & & & & & & & & & & & & \\
\hline Seuil de significativité & & & & & & & & & & & & \\
\hline Sexe du chef de ménage & & & & & & & & & & & & \\
\hline Hommes & 82,5 & 17,5 & 100,0 & 2571086 & 68,9 & 31,1 & 100,0 & 5340943 & 73,3 & 26,7 & 100,0 & 7912029 \\
\hline Femmes & 81,0 & 19,0 & 100,0 & 1630964 & 69,6 & 30,4 & 100,0 & 3574603 & 73,1 & 26,9 & 100,0 & 5205567 \\
\hline V de Cramer & & & & & & & & & & & & \\
\hline Seuil de significativité & & & & & & & & & & & & \\
\hline Nombre de malades & & & & & & & & & & & & \\
\hline Aucun malade & 83,6 & 16,4 & 100,0 & 3065257 & 70,2 & 29,8 & 100,0 & 6484593 & 74,5 & 25,5 & 100,0 & 9549850 \\
\hline 1 malade & 74,2 & 25,8 & 100,0 & 644534 & 67,8 & 32,2 & 100,0 & 1355706 & 69,9 & 30,1 & 100,0 & 2000240 \\
\hline 2 malades & 78,5 & 21,5 & 100,0 & 253830 & 66,9 & 33,1 & 100,0 & 600509 & 70,4 & 29,6 & 100,0 & 854339 \\
\hline 3 malades et plus & 85,2 & 14,8 & 100,0 & 238429 & 61,9 & 38,1 & 100,0 & 474738 & 69,7 & 30,3 & 100,0 & 713167 \\
\hline D de Somers & & & & & & & & & & & & \\
\hline Seuil de significativité & & & & & & & & & & & & \\
\hline Nombre d'enfants de 6- & & & & & & & & & & & & \\
\hline $1-2$ & 80,0 & 20,0 & 100,0 & 1690154 & 68,3 & 31,7 & 100,0 & 4128182 & 71,7 & 28,3 & 100,0 & 5818336 \\
\hline $3-4$ & 82,7 & 17,3 & 100,0 & 1835647 & 71,5 & 28,5 & 100,0 & 3824359 & 75,1 & 24,9 & 100,0 & 5660006 \\
\hline 5 et plus & 84,6 & 15,4 & 100,0 & 676577 & 63,5 & 36,5 & 100,0 & 962677 & 72,2 & 27,8 & 100,0 & 1639254 \\
\hline D de Somers & & & & & & & & & & & & \\
\hline Seuil de significativité & & & & & & & & & & & & \\
\hline Distance à l'école prima & & & & & & & & & & & & \\
\hline Moins $2 \mathrm{Km}$ & 81,9 & 18,1 & 100,0 & 4098056 & 72,9 & 27,1 & 100,0 & 6566974 & 76,4 & 23,6 & 100,0 & 10665030 \\
\hline $2-5 \mathrm{Km}$ & 84,4 & 15,6 & 100,0 & 93989 & 65,5 & 34,5 & 100,0 & 1426120 & 66,7 & 33,3 & 100,0 & 1520108 \\
\hline plus de $5 \mathrm{Km}$ & 77,3 & 22,7 & 100,0 & 9452 & 48,1 & 51,9 & 100,0 & 923006 & 48,4 & 51,6 & 100,0 & 932458 \\
\hline D de Somers & & & & & & & & & & & & \\
\hline Seuil de significativité & & & & & & & & & & & & \\
\hline Principal handicap & & & & & & & & & & & & \\
\hline Aucun & 82,2 & 17,8 & 100,0 & 4136677 & 69,5 & 30,5 & 100,0 & 8816017 & 73,6 & 26,4 & 100,0 & 12952694 \\
\hline Sourd & 83,3 & 16,7 & 100,0 & 11095 & 30,0 & 70,0 & 100,0 & 15088 & 52,6 & 47,4 & 100,0 & 26183 \\
\hline Muet & 4,0 & 96,0 & 100,0 & 3644 & & 100,0 & 100,0 & 14130 & 0,8 & 99,2 & 100,0 & 17774 \\
\hline Mal-voyant & 72,4 & 27,6 & 100,0 & 7007 & 43,9 & 56,1 & 100,0 & 5593 & 59,7 & 40,3 & 100,0 & 12600 \\
\hline Aveugle & - & - & - & - & - & 100,0 & 100,0 & 1816 & & 100,0 & 100,0 & 1816 \\
\hline Handicap mbre sup. & 88,8 & 11,2 & 100,0 & 8892 & 67,9 & 32,1 & 100,0 & 16194 & 75,3 & 24,7 & 100,0 & 25086 \\
\hline Handicap mbre inf. & 87,2 & 12,8 & 100,0 & 12141 & 53,0 & 47,0 & 100,0 & 25294 & 64,1 & 35,9 & 100,0 & 37435 \\
\hline Déficient mental & 17,3 & 82,7 & 100,0 & 9389 & 3,9 & 96,1 & 100,0 & 12161 & 9,8 & 90,2 & 100,0 & 21550 \\
\hline Autre & 61,8 & 38,2 & 100,0 & 13204 & 48,3 & 51,7 & 100,0 & 9254 & 56,3 & 43,7 & 100,0 & 22458 \\
\hline V de Cramer & & & & & & & & & & & & \\
\hline Seuil de significativité & & & & & & & & & & & 00 & \\
\hline Total & 81,9 & 18,1 & 100,0 & 4202049 & 69,2 & 30,8 & 100,0 & 8915547 & 73,2 & 26,8 & 100,0 & 13117596 \\
\hline
\end{tabular}

Source : Données de l'enquête auprès des ménages, EADE-RDC 2012

- Effectifs faibles 
Tableau A26 : Répartition (en \%) de la population de 12-17 ans selon le statut d'exclusion par caractéristiques

\begin{tabular}{|c|c|c|c|c|c|c|c|c|c|c|c|c|}
\hline \multirow{2}{*}{ Caractéristiques } & \multicolumn{4}{|c|}{ Urbain } & \multicolumn{4}{|c|}{ Rural } & \multicolumn{4}{|c|}{ Total } \\
\hline & Non & Oui & Total & Total & Non & Oui & Total & Total & Non & Oui & Total & Total \\
\hline \multicolumn{13}{|c|}{ Sexe du membre du ménage } \\
\hline Garçons & 90,3 & 9,7 & 100,0 & 1728503 & 85,5 & 14,5 & 100,0 & 3261895 & 87,1 & 12,9 & 100,0 & 4990398 \\
\hline Filles & 82,5 & 17,5 & 100,0 & 1705560 & 73,3 & 26,7 & 100,0 & 2921644 & 76,6 & 23,4 & 100,0 & 4627204 \\
\hline V de Cramer & \multicolumn{4}{|c|}{0,083} & \multicolumn{4}{|c|}{0,164} & & & & \\
\hline Seuil de significativité & & & & & & 0 , & & & & & & \\
\hline Revenu mensuel du mér & & & & & & & & & & & & \\
\hline Moins de 50 \$US & 74,7 & 25,3 & 100,0 & 583752 & 76,2 & 23,8 & 100,0 & 3835967 & 76,0 & 24,0 & 100,0 & 4419719 \\
\hline 50 à 100 \$US & 86,2 & 13,8 & 100,0 & 884527 & 85,6 & 14,4 & 100,0 & 1734343 & 85,8 & 14,2 & 100,0 & 2618870 \\
\hline 101 à 200 \$US & 86,7 & 13,3 & 100,0 & 950391 & 85,5 & 14,5 & 100,0 & 449747 & 86,3 & 13,7 & 100,0 & 1400138 \\
\hline Plus de 200 \$US & 93,1 & 6,9 & 100,0 & 1015393 & 82,5 & 17,5 & 100,0 & 163482 & 91,6 & 8,4 & 100,0 & 1178875 \\
\hline D de Somers & & & & & & & & & & & & \\
\hline Seuil de significativité & & & & & & 0 , & & & & & & \\
\hline Statut familial & & & & & & & & & & & & \\
\hline Enfant du CM & 89,6 & 10,4 & 100,0 & 2449785 & 82,2 & 17,8 & 100,0 & 4914670 & 84,7 & 15,3 & 100,0 & 7364455 \\
\hline Autre parent & 78,7 & 21,3 & 100,0 & 962054 & 70,4 & 29,6 & 100,0 & 1240237 & 74,0 & 26,0 & 100,0 & 2202291 \\
\hline Sans parenté & 75,5 & 24,5 & 100,0 & 22224 & 48,8 & 51,2 & 100,0 & 28632 & 60,5 & 39,5 & 100,0 & 50856 \\
\hline D de Somers & & & & & & 0 , & & & & & & \\
\hline Seuil de significativité & & & & & & 0 & & & & & & \\
\hline statut d'orphelin & & & & & & & & & & & & \\
\hline Non orphelin & 88,6 & 11,4 & 100,0 & 2800514 & 81,7 & 18,3 & 100,0 & 5181990 & 84,1 & 15,9 & 100,0 & 7982505 \\
\hline Orphelin de mère & 75,5 & 24,5 & 100,0 & 116092 & 73,7 & 26,3 & 100,0 & 250451 & 74,3 & 25,7 & 100,0 & 366543 \\
\hline Orphelin de père & 75,2 & 24,8 & 100,0 & 391611 & 70,1 & 29,9 & 100,0 & 587818 & 72,1 & 27,9 & 100,0 & 979428 \\
\hline Orphelin complet & 81,8 & 18,2 & 100,0 & 126875 & 60,9 & 39,1 & 100,0 & 162251 & 70,0 & 30,0 & 100,0 & 289126 \\
\hline V de Cramer & & & & & & 0 , & & & & & & \\
\hline Seuil de significativité & & & & & & 0 , & & & & & & \\
\hline Niveau d'instruction du & & & & & & & & & & & & \\
\hline Aucun & 85,0 & 15,0 & 100,0 & 1542669 & 79,3 & 20,7 & 100,0 & 2998536 & 81,2 & 18,8 & 100,0 & 4541205 \\
\hline Primaire & 74,6 & 25,4 & 100,0 & 121833 & 65,4 & 34,6 & 100,0 & 1002947 & 66,4 & 33,6 & 100,0 & 1124780 \\
\hline Secondaire+ & 88,5 & 11,5 & 100,0 & 1769561 & 86,8 & 13,2 & 100,0 & 2182056 & 87,6 & 12,4 & 100,0 & 3951617 \\
\hline D de Somers & & & & & & & & & & & & \\
\hline Seuil de significativité & & & & & & 0 , & & & & & & \\
\hline Sexe du chef de ménage & & & & & & & & & & & & \\
\hline Hommes & 86,4 & 13,6 & 100,0 & 2179814 & 79,8 & 20,2 & 100,0 & 4041518 & 82,1 & 17,9 & 100,0 & 6221332 \\
\hline Femmes & 86,4 & 13,6 & 100,0 & 1254248 & 79,4 & 20,6 & 100,0 & 2142021 & 82,0 & 18,0 & 100,0 & 3396270 \\
\hline V de Cramer & & & & & & & & & & & & \\
\hline Seuil de significativité & & & & & & 0 & & & & & & \\
\hline Nombre de malades & & & & & & & & & & & & \\
\hline Aucun malade & 87,1 & 12,9 & 100,0 & 2546447 & 80,3 & 19,7 & 100,0 & 4344425 & 82,8 & 17,2 & 100,0 & 6890872 \\
\hline 1 malade & 87,5 & 12,5 & 100,0 & 506130 & 79,2 & 20,8 & 100,0 & 1016632 & 81,9 & 18,1 & 100,0 & 1522762 \\
\hline 2 malades & 84,6 & 15,4 & 100,0 & 206432 & 75,4 & 24,6 & 100,0 & 454316 & 78,3 & 21,7 & 100,0 & 660748 \\
\hline 3 malades et plus & 75,8 & 24,2 & 100,0 & 175055 & 78,9 & 21,1 & 100,0 & 368165 & 77,9 & 22,1 & 100,0 & 543220 \\
\hline D de Somers & & & & & & & & & & & & \\
\hline Seuil de significativité & & & & & & 0 , & & & & & & \\
\hline Nombre d'enfants de 6-1 & & & & & & & & & & & & \\
\hline $1-2$ & 81,5 & 18,5 & 100,0 & 1212484 & 76,0 & 24,0 & 100,0 & 2223289 & 77,9 & 22,1 & 100,0 & 3435773 \\
\hline $3-4$ & 88,4 & 11,6 & 100,0 & 1575919 & 82,3 & 17,7 & 100,0 & 2943211 & 84,4 & 15,6 & 100,0 & 4519130 \\
\hline 5 et plus & 90,9 & 9,1 & 100,0 & 645661 & 80,4 & 19,6 & 100,0 & 1017038 & 84,5 & 15,5 & 100,0 & 1662699 \\
\hline D de Somers & & & & & & & & & & & & \\
\hline Seuil de significativité & & & & & & 0, & & & & & & \\
\hline Distance à l'école secon & & & & & & & & & & & & \\
\hline Moins $2 \mathrm{Km}$ & 87,3 & 12,7 & 100,0 & 3058376 & 84,5 & 15,5 & 100,0 & 3087202 & 85,9 & 14,1 & 100,0 & 6145578 \\
\hline $2-5 \mathrm{Km}$ & 79,9 & 20,1 & 100,0 & 308647 & 80,1 & 19,9 & 100,0 & 1263719 & 80,0 & 20,0 & 100,0 & 1572366 \\
\hline $5-10 \mathrm{Km}$ & 72,2 & 27,8 & 100,0 & 55921 & 77,9 & 22,1 & 100,0 & 1176205 & 77,6 & 22,4 & 100,0 & 1232126 \\
\hline plus de $10 \mathrm{Km}$ & 88,8 & 11,2 & 100,0 & 12518,0 & 59,7 & 40,3 & 100,0 & 655015,0 & 60,3 & 39,7 & 100,0 & 667533,0 \\
\hline D de Somers & & & & & & & & & & & & \\
\hline Seuil de significativité & & & & & & & & & & & & \\
\hline Principal handicap & & & & & & & & & & & & \\
\hline Aucun & 86,8 & 13,2 & 100 & 3388785 & 80,2 & 19,8 & 100 & 6093720 & 82,6 & 17,4 & 100 & 9482505 \\
\hline Sourd & 3,7 & 96,3 & 100 & 3501 & 25,1 & 74,9 & 100 & 11383 & 20 & 80 & 100 & 14884 \\
\hline Muet & 49,9 & 50,1 & 100 & 4201 & 33 & 67 & 100 & 10348 & 37,9 & 62,1 & 100 & 14549 \\
\hline Mal-voyant & 99,9 & 0,1 & 100 & 3534 & 100 & - & 100 & 2334 & 100 & - & 100 & 5868 \\
\hline Aveugle & - & - & - & - & - & 100 & 100 & 4668 & & 100 & 100 & 4668 \\
\hline Handicap mbre sup. & 67,7 & 32,3 & 100 & 11443 & 54,9 & 45,1 & 100 & 10996 & 61,4 & 38,6 & 100 & 22439 \\
\hline Handicap mbre inf. & 40,4 & 59,6 & 100 & 6974 & 46,8 & 53,2 & 100 & 14824 & 44,7 & 55,3 & 100 & 21798 \\
\hline Déficient mental & 29,9 & 70,1 & 100 & 5380 & 37,5 & 62,5 & 100 & 26507 & 36,2 & 63,8 & 100 & 31887 \\
\hline Autre & 65,7 & 34,3 & 100 & 10246 & 78,8 & 21,2 & 100 & 8758 & 71,8 & 28,2 & 100 & 19004 \\
\hline V de Cramer & & & & & & 0 , & & & & & & \\
\hline Seuil de significativité & & & & & & & & & & & & \\
\hline Total & 86,4 & 13,6 & 100 & 3434064 & 79,7 & 20,3 & 100 & 6183538 & 82,1 & 17,9 & 100 & 9617602 \\
\hline
\end{tabular}


Tableau A27 : Modèles logistiques urbain-rural (6-11 ans et 12-17 ans)

\begin{tabular}{|c|c|c|c|c|c|c|}
\hline \multirow{2}{*}{ Variables } & \multicolumn{3}{|c|}{$6-11$ ans } & \multicolumn{3}{|c|}{$12-17$ ans } \\
\hline & Ensemble & Urbain & Rural & Ensemble & Urbain & Rural \\
\hline \multicolumn{7}{|l|}{ Sexe } \\
\hline Garçons & 1,00 & 1,00 & 1,00 & 1,00 & 1,00 & 1,00 \\
\hline Filles & $1,14^{* *}$ & 1,07 & $1,18^{* *}$ & $1,99^{\text {*** }}$ & $1,53^{* * *}$ & $2,38^{* * * *}$ \\
\hline \multicolumn{7}{|l|}{ Statut d'orphelin } \\
\hline 2 parents vivants & 1,00 & 1,00 & 1,00 & 1,00 & 1,00 & 1,00 \\
\hline Mère vivant et Père décédé & 0,93 & 1,16 & 0,81 & 1,41 & 1,41 & 1,37 \\
\hline Mère décédée et Père vivant & $1,31^{* *}$ & 1,17 & $1,45^{* * *}$ & $1,89^{* * * *}$ & $1,96^{* * * *}$ & $1,88^{* * * *}$ \\
\hline 2 parents décédés & 0,98 & 0,77 & 1,26 & $1,64^{*}$ & 1,60 & 1,71 \\
\hline \multicolumn{7}{|l|}{ Lien de parenté avec le CM } \\
\hline Enfant du $C M$ & 1,00 & 1,00 & 1,00 & 1,00 & 1,00 & 1,00 \\
\hline Enfant apparenté & $1,23^{* * *}$ & $1,55^{\text {**** }}$ & 1,07 & $1,63^{\text {*** }}$ & $2,03^{* * *}$ & $1,30 *$ \\
\hline Enfant non apparenté & $3,16^{*}$ & - & $5,87^{*}$ & 2,26 & 4,15 & - \\
\hline \multicolumn{7}{|l|}{ Handicap de l'enfant } \\
\hline Aucun & 1,00 & 1,00 & 1,00 & 1,00 & 1,00 & 1,00 \\
\hline Hand, sensoriel & $9,14^{* * *}$ & $11,22^{* * * *}$ & $8,89^{* * * *}$ & $30,32^{* * *}$ & $53,13^{* * *}$ & $14,24^{* *}$ \\
\hline Hand, visuel & 1,93 & 1,626 & 2,33 & $3,90^{*}$ & 2,71 & 6,64 \\
\hline Hand, moteur & 1,67 & 1,129 & 1,99 & $4,71^{* * * *}$ & $6,60^{* * *}$ & $3,56^{*}$ \\
\hline Déficient mental & $45,87^{* * * *}$ & $81,97^{* * * *}$ & $20,53^{* * *}$ & $31,77^{* * * *}$ & $58,89^{*}$ & $27,56^{\text {**** }}$ \\
\hline Autres & $3,25^{*}$ & 3,64 & 2,87 & $6,57^{* * *}$ & $9,32^{* *}$ & 3,95 \\
\hline \multicolumn{7}{|l|}{ Niveau d'instruction du CM } \\
\hline Aucun & 1,00 & 1,00 & 1,00 & 1,00 & 1,00 & 1,00 \\
\hline Primaire & 1,09 & 1,33 & 1,00 & $1,89^{* * * *}$ & $2,34^{* *}$ & $1,82^{* * * *}$ \\
\hline Secondaire & $0,57^{* * *}$ & $0,74^{* * *}$ & $0,50^{* * * *}$ & $0,67^{\text {*** }}$ & 0,81 & $0,58^{* * * *}$ \\
\hline Supérieur & $0,30^{* * *}$ & $0,35^{* * * *}$ & 0,49 & $0,16^{* * *}$ & $0,17^{* * *}$ & 0,18 \\
\hline \multicolumn{7}{|l|}{ Sexe du CM } \\
\hline Hommes & 1,00 & 1,00 & 1,00 & 1,00 & 1,00 & 1,00 \\
\hline Femmes & 0,94 & 1,11 & $0,86^{* * *}$ & 0,89 & 0,85 & 0,91 \\
\hline \multicolumn{7}{|l|}{ Religion du CM } \\
\hline Catholique & 1,00 & 1,00 & 1,00 & 1,00 & 1,00 & 1,00 \\
\hline Protestant & 1,02 & 1,10 & 1,00 & 1,11 & 1,14 & 1,10 \\
\hline Kimbanguiste & 0,86 & 1,38 & 0,76 & 0,75 & 0,88 & 0,71 \\
\hline Eglises de réveil & 0,97 & $1,32^{*}$ & $0,83^{*}$ & 1,06 & 1,35 & 0,79 \\
\hline Chrétiens indépendants & 0,74 & 0,97 & 0,61 & 1,09 & 1,32 & 0,76 \\
\hline Témoins de Jéhovah & $1,17^{*}$ & 1,25 & 1,173 & $0,71^{*}$ & 0,86 & $0,64^{* *}$ \\
\hline Musulman & 0,76 & 1,02 & 0,69 & 0,59 & 1,38 & $0,40^{*}$ \\
\hline Autres & 1,20 & 1,18 & 1,21 & 1,35 & 1,60 & 1,18 \\
\hline \multicolumn{7}{|l|}{ Revenu du ménage } \\
\hline$<=50 \$$ & 1,00 & 1,00 & 1,00 & 1,00 & 1,00 & 1,00 \\
\hline $50-100 \$$ & $0,68^{* * *}$ & $0,71^{* * * *}$ & $0,71^{* * * *}$ & $0,67^{* * * *}$ & $0,60^{* *}$ & $0,69^{* * * *}$ \\
\hline $101-200 \$$ & $0,41^{* * *}$ & $0,41^{\text {**** }}$ & $0,56^{* * * *}$ & $0,52^{\text {*** }}$ & $0,44^{* * * *}$ & $0,54^{* *}$ \\
\hline $201-500 \$$ & $0,22^{* * *}$ & $0,22^{* * * *}$ & $0,40^{* * * *}$ & $0,49^{* * *}$ & $0,37^{* * * *}$ & 0,77 \\
\hline$>=500 \$$ & $0,13^{* * * *}$ & $0,10^{* * * *}$ & - & $0,16^{* * * *}$ & $0,11^{* * *}$ & 0,73 \\
\hline \multicolumn{7}{|l|}{ Nombre d'enfants de 6-17 ans } \\
\hline $1-2$ & 1,00 & 1,00 & 1,00 & 1,00 & 1,00 & 1,00 \\
\hline $3-4$ & $1,26^{* * *}$ & $1,47^{* * * *}$ & $1,17^{*}$ & 1,09 & 0,94 & 1,23 \\
\hline 5 et plus & 1,25 & $1,60^{*}$ & 1,00 & 0,59 & 0,89 & 0,22 \\
\hline \multicolumn{7}{|l|}{ Nombre de malade } \\
\hline aucun & 1,00 & 1,00 & 1,00 & 1,00 & 1,00 & 1,00 \\
\hline un & 1,06 & 0,97 & 1,09 & 1,05 & 0,95 & 1,13 \\
\hline deux & 1,17 & 1,15 & 1,19 & 1,12 & 0,93 & 1,29 \\
\hline trois et plus & 1,02 & 0,77 & 1,17 & 1,04 & 1,41 & 0,88 \\
\hline \multicolumn{7}{|l|}{ Distance à l'école (primaire) } \\
\hline$<2$ & 1,00 & 1,00 & 1,00 & nc & nc & nc \\
\hline $2-5$ & $1,48^{* * * *}$ & $1,50^{* *}$ & $1,43^{* * * *}$ & $\mathrm{nc}$ & nc & nc \\
\hline 5 et plus & $2,34^{* * * *}$ & 1,35 & $2,24^{* * * *}$ & $\mathrm{nc}$ & $\mathrm{nc}$ & $\mathrm{nc}$ \\
\hline Distance à l'école (secondaire) & & & & & & \\
\hline$<2$ & $\mathrm{nc}$ & $\mathrm{nc}$ & $\mathrm{nc}$ & 1,00 & 1,00 & 1,00 \\
\hline $2-5$ & nc & nc & nc & 0,90 & 0,71 & 1,07 \\
\hline $5-10$ & nc & nc & $\mathrm{nc}$ & 1,09 & 0,93 & 1,19 \\
\hline 10 et plus & $\mathrm{nc}$ & $\mathrm{nc}$ & $\mathrm{nc}$ & $1,75^{\text {**** }}$ & 1,16 & $2,00^{* * * *}$ \\
\hline Effectifs de l'échantillon (n) & 12259 & 5270 & 6989 & 9202 & 4385 & 4817 \\
\hline Pseudo $\mathrm{R}^{2}$ & $0,078^{* * * *}$ & $0,084^{* * * *}$ & $0,049^{* * * *}$ & $0,110^{* * * *}$ & $0,125^{* * * *}$ & $0,103^{* * * *}$ \\
\hline
\end{tabular}

Notes : - = effectifs faibles $\mathrm{nc}=$ non concerné 
Tableau A28 : Modèles logistiques au niveau province (6-11 ans)

\begin{tabular}{|c|c|c|c|c|c|c|c|c|c|c|c|}
\hline Variables & Kinshasa & Bas congo & Bandundu & Equateur & $\begin{array}{l}\text { Province } \\
\text { orientale }\end{array}$ & Nord kivu & Maniema & Sud kivu & Katanga & $\begin{array}{l}\text { Kasai } \\
\text { oriental }\end{array}$ & $\begin{array}{l}\text { Kasai } \\
\text { occidental }\end{array}$ \\
\hline \multicolumn{12}{|l|}{ Sexe } \\
\hline Garçons & 1,00 & 1,00 & 1,00 & 1,00 & 1,00 & 1,00 & 1,00 & 1,00 & 1,00 & 1,00 & 1,00 \\
\hline Filles & 0,94 & 1,01 & 0,81 & 1,17 & 1,07 & 1,07 & 1,23 & $1,50^{*}$ & 0,89 & 1,22 & $1,74^{\star \star \star}$ \\
\hline \multicolumn{12}{|l|}{ Statut d'orphelin } \\
\hline 2 parents vivants & 1,00 & 1,00 & 1,00 & 1,00 & 1,00 & 1,00 & 1,00 & 1,00 & 1,00 & 1,00 & 1,00 \\
\hline Père décédé \& Mère vivante & 0,93 & 0,28 & 1,96 & 0,85 & 1,34 & 0,53 & 2,03 & 0,61 & 1,24 & 1,08 & 0,75 \\
\hline Mère décédée \& Père vivant & $2,11^{*}$ & 0,99 & 1,32 & 1,63 & 1,06 & 1,08 & 1,61 & 1,12 & 1,34 & 1,11 & 1,50 \\
\hline 2 parents décédés & 0,68 & 1,24 & 1,16 & 0,35 & 1,76 & 1,35 & 1,19 & 1,15 & 0,51 & 0,77 & 0,65 \\
\hline \multicolumn{12}{|l|}{ Lien de parenté avec le CM } \\
\hline Enfant du CM & 1,00 & 1,00 & 1,00 & 1,00 & 1,00 & 1,00 & 1,00 & 1,00 & 1,00 & 1,00 & 1,00 \\
\hline Enfant apparenté & $1,68^{*}$ & 0,93 & 0,97 & $2,37^{\star * *}$ & 1,07 & $1,46^{*}$ & 0,60 & 1,01 & $1,98^{\star \star \star}$ & 1,32 & 0,97 \\
\hline Enfant non apparenté & - & - & 2,10 & - & - & - & - & - & - & - & - \\
\hline \multicolumn{12}{|l|}{ Niveau d'instruction du CM } \\
\hline Aucun & 1,00 & 1,00 & 1,00 & 1,00 & 1,00 & 1,00 & 1,00 & 1,00 & 1,00 & 1,00 & 1,00 \\
\hline Primaire & 0,80 & 0,81 & 0,72 & 0,88 & 1,38 & 1,07 & 0,81 & $0,37^{\star *}$ & 1,401 & 1,323 & 0,891 \\
\hline Secondaire ou plus & $0,61^{*}$ & $0,44^{* * *}$ & $0,45^{\star \star \star}$ & $0,48^{\star * *}$ & $0,62^{*}$ & $0,52^{* * \star}$ & 0,68 & $0,42^{\star * *}$ & $0,58^{\star * *}$ & $0,58^{* *}$ & $0,61^{* *}$ \\
\hline \multicolumn{12}{|l|}{ Sexe du CM } \\
\hline Hommes & 1,00 & 1,00 & 1,00 & 1,00 & 1,00 & 1,00 & 1,00 & 1,00 & 1,00 & 1,00 & 1,00 \\
\hline Femmes & 1,31 & 0,93 & 0,90 & 0,89 & 0,95 & 0,89 & 0,82 & 1,21 & 1,00 & 0,87 & 0,98 \\
\hline \multicolumn{12}{|l|}{ Religion du CM } \\
\hline Catholique & 1,00 & 1,00 & 1,00 & 1,00 & 1,00 & 1,00 & 1,00 & 1,00 & 1,00 & 1,00 & 1,00 \\
\hline Protestant & 0,83 & 1,25 & 1,09 & 1,09 & 1,20 & $1,31^{*}$ & 1,30 & 1,02 & 0,74 & 1,06 & 1,08 \\
\hline Kimbanguiste & $3,34^{*}$ & 0,61 & 1,02 & 1,18 & - & - & 1,94 & - & 0,68 & 0,32 & 1,37 \\
\hline Eglises de réveil & 1,14 & 0,60 & 0,96 & 0,88 & $3,13^{\star \star *}$ & 0,65 & 1,37 & 1,93 & $1,67^{\star *}$ & 1,18 & 0,81 \\
\hline Chrétiens indépendants & 0,85 & - & - & 0,35 & 1,85 & 0,81 & - & - & 1,18 & 0,91 & 1,22 \\
\hline Témoins de Jéhovah & 1,57 & 0,79 & 0,70 & 1,82 & 1,14 & 1,40 & 1,88 & 1,46 & 0,91 & $2,10^{\star *}$ & 0,92 \\
\hline Musulman & 0,86 & - & - & - & 1,50 & 0,79 & 1,14 & 0,60 & 1,43 & 3,22 & 0,71 \\
\hline Autres & 2,26 & 1,12 & 0,73 & 0,80 & 1,39 & 0,71 & 4,56 & 1,21 & $1,61^{*}$ & 1,62 & 0,70 \\
\hline \multicolumn{12}{|l|}{ Revenu du ménage } \\
\hline$<=50 \$$ & 1,00 & 1,00 & 1,00 & 1,00 & 1,00 & 1,00 & 1,00 & 1,00 & 1,00 & 1,00 & 1,00 \\
\hline $50-100 \$$ & 0,98 & 0,70 & $0,52^{\star *}$ & 0,79 & 1,08 & $0,48^{\star \star \star}$ & $0,64^{*}$ & $0,42^{* * *}$ & $0,65^{\star \star \star}$ & 0,96 & $0,75^{\star}$ \\
\hline $101-200 \$$ & $0,39^{*}$ & $0,31^{\star * *}$ & $0,26^{\star \star}$ & $0,21^{\star * *}$ & 0,74 & $0,37^{\star \star \star}$ & 0,46 & $0,23^{\star \star \star}$ & $0,41^{\star \star \star}$ & $0,43^{\star \star \star}$ & $0,39^{\star \star *}$ \\
\hline$>200 \$$ & $0,19^{\star \star \star}$ & $0,18^{\star \star \star}$ & 0,67 & 0,34 & 0,67 & $0,20^{\star \star \star}$ & 1,05 & $0,04^{* \star *}$ & $0,10^{* \star *}$ & $0,14^{*}$ & $0,07^{\star \star}$ \\
\hline \multicolumn{12}{|l|}{ Nombre d'enfants de 6-17 ans } \\
\hline $1-2$ & 1,00 & 1,00 & 1,00 & 1,00 & 1,00 & 1,00 & 1,00 & 1,00 & 1,00 & 1,00 & 1,00 \\
\hline 3-4 & 1,09 & 1,13 & 0,82 & 0,74 & 0,91 & 0,92 & 1,10 & 1,08 & 1,07 & 1,03 & 0,90 \\
\hline 5 et plus & 1,16 & 0,88 & 0,64 & $2,82^{* * *}$ & 0,99 & $1,50^{*}$ & $5,02^{* \star}$ & 1,19 & 1,14 & 1,48 & 0,83 \\
\hline \multicolumn{12}{|l|}{ Nombre de malades } \\
\hline aucun & 1,00 & 1,00 & 1,00 & 1,00 & 1,00 & 1,00 & 1,00 & 1,00 & 1,00 & 1,00 & 1,00 \\
\hline un & 0,98 & 1,15 & 1,31 & 0,97 & 0,98 & 1,10 & $1,66^{*}$ & 0,72 & 1,37 & $1,56^{*}$ & 0,79 \\
\hline deux & 0,47 & 1,85 & 1,25 & 0,73 & $2,09^{\star *}$ & 1,18 & 1,55 & 0,88 & 1,24 & $2,01^{* *}$ & 0,90 \\
\hline trois et plus & 1,41 & 1,28 & 1,01 & 0,81 & 1,80 & 1,00 & 0,72 & 1,35 & $1,65^{*}$ & 1,07 & 0,84 \\
\hline \multicolumn{12}{|l|}{ Distance à l'école (primaire) } \\
\hline$<2$ & 1,00 & 1,00 & 1,00 & 1,00 & 1,00 & 1,00 & 1,00 & 1,00 & 1,00 & 1,00 & 1,00 \\
\hline $2-5$ & 0,69 & 0,83 & 0,56 & $2,04^{\star *}$ & $1,94^{\star \star}$ & 1,02 & 1,60 & 1,51 & $1,73^{\star \star}$ & 1,31 & $1,88^{\star \star \star}$ \\
\hline 5 et plus & - & 0,93 & $2,39^{\star \star *}$ & 0,91 & $11,51^{* \star *}$ & $3,67^{\star * *}$ & $3,704^{\star * \star}$ & $4,47^{\star * *}$ & $3,14^{\star * *}$ & 2,26 & $2,45^{\star * *}$ \\
\hline Effectifs de l'échantillon (n) & 1032 & 963 & 1023 & 858 & 897 & 1410 & 795 & 1085 & 1771 & 1096 & 1329 \\
\hline Pseudo R2 & 0,143 & 0,067 & 0,094 & 0,079 & 0,114 & 0,078 & 0,075 & 0,131 & 0,127 & 0,075 & 0,078 \\
\hline
\end{tabular}


Tableau A29 : Modèles logistiques au niveau province (12-17 ans)

\begin{tabular}{|c|c|c|c|c|c|c|c|c|c|c|c|}
\hline Variables & Kinshasa & Bas congo & Bandundu & Equateur & $\begin{array}{l}\text { Province } \\
\text { orientale }\end{array}$ & Nord kivu & Maniema & Sud kivu & Katanga & $\begin{array}{l}\text { Kasai } \\
\text { oriental }\end{array}$ & $\begin{array}{l}\text { Kasai } \\
\text { occidental }\end{array}$ \\
\hline \multicolumn{12}{|l|}{ Sexe } \\
\hline Garçons & 1,00 & 1,00 & 1,00 & 1,00 & 1,00 & 1,00 & 1,00 & 1,00 & 1,00 & 1,00 & 1,00 \\
\hline Filles & 1,064 & $2,16^{\star \star *}$ & $2,03^{*}$ & 1,28 & $1,98^{\star \star}$ & $1,93^{\star \star \star}$ & 1,57 & 1,41 & $2,41^{* \star \star}$ & $5,48^{\star \star \star}$ & $3,02^{\star * *}$ \\
\hline \multicolumn{12}{|l|}{ Statut d'orphelin } \\
\hline 2 parents vivants & 1,00 & 1,00 & 1,00 & 1,00 & 1,00 & 1,00 & 1,00 & 1,00 & 1,00 & 1,00 & 1,00 \\
\hline Père décédé \& Mère vivante & 2,06 & $2,58^{*}$ & $5,34^{*}$ & 2,98 & 1,27 & 1,08 & 1,04 & 1,51 & 1,33 & 2,83 & 1,53 \\
\hline Mère décédée \& Père vivant & $3,21^{* * *}$ & $2,17^{*}$ & 0,88 & 0,86 & $2,70^{\star *}$ & 1,45 & 0,91 & 1,38 & $2,11^{* *}$ & 1,63 & $2,75^{\star \star *}$ \\
\hline 2 parents décédés & 0,87 & 2,76 & 4,29 & 2,51 & 1,32 & 1,08 & 1,30 & 1,24 & 1,29 & $3,56^{*}$ & $2,78^{*}$ \\
\hline \multicolumn{12}{|l|}{ Lien de parenté avec le CM } \\
\hline Enfant du CM & 1,00 & 1,00 & 1,00 & 1,00 & 1,00 & 1,00 & 1,00 & 1,00 & 1,00 & 1,00 & 1,00 \\
\hline Enfant apparenté & $2,42^{\star \star \star}$ & 1,33 & 1,55 & 1,53 & 0,87 & $1,73^{\star *}$ & $2,86^{*}$ & 1,13 & $1,80^{\star *}$ & $2,50^{\star \star \star}$ & $2,20^{\star \star}$ \\
\hline Enfant non apparenté & 3,83 & 2,84 & - & - & - & $10,70^{*}$ & - & $16,37^{* *}$ & - & 21,85 & 5,12 \\
\hline \multicolumn{12}{|l|}{ Niveau d'instruction du CM } \\
\hline Aucun & 1,00 & 1,00 & 1,00 & 1,00 & 1,00 & 1,00 & 1,00 & 1,00 & 1,00 & 1,00 & 1,00 \\
\hline Primaire & 3,75 & 1,60 & 0,31 & 2,38 & 1,37 & $1,73^{\star \star}$ & $3,54^{\star}$ & 1,50 & 1,26 & 2,18 & 1,30 \\
\hline Secondaire ou plus & $0,61^{*}$ & $0,55^{*}$ & 0,65 & 0,69 & 0,90 & $10,70^{*}$ & 0,58 & $0,62^{*}$ & $0,46^{* \star *}$ & 1,29 & 0,71 \\
\hline \multicolumn{12}{|l|}{ Sexe du CM } \\
\hline Hommes & 1,00 & 1,00 & 1,00 & 1,00 & 1,00 & 1,00 & 1,00 & 1,00 & 1,00 & 1,00 & 1,00 \\
\hline Femmes & 0,71 & 0,72 & $4,27^{\star \star \star}$ & 0,99 & 0,67 & $1,57^{\star \star}$ & 0,54 & 0,66 & $0,57^{\star \star}$ & 0,60 & $0,56^{\star}$ \\
\hline \multicolumn{12}{|l|}{ Religion du CM } \\
\hline Catholique & 1,00 & 1,00 & 1,00 & 1,00 & 1,00 & 1,00 & 1,00 & 1,00 & 1,00 & 1,00 & 1,00 \\
\hline Protestant & 1,27 & 1,18 & 1,69 & 1,19 & 0,78 & 1,12 & 1,55 & 1,42 & 0,82 & 1,90 & 0,92 \\
\hline Kimbanguiste & 0,43 & 0,87 & 2,08 & 3,15 & - & - & 4,02 & - & 0,94 & 0,45 & 0,85 \\
\hline Eglises de réveil & 1,09 & 1,69 & 1,96 & 1,07 & $3,05^{\star \star}$ & 0,95 & 1,54 & $2,83^{*}$ & $2,25^{\star \star}$ & 1,44 & 0,93 \\
\hline Chrétiens indépendants & 0,63 & - & 3,59 & 1,94 & 1,34 & 0,14 & - & $4,41^{*}$ & 2,48 & 0,42 & 0,80 \\
\hline Témoins de Jéhovah & 1,99 & 0,53 & 0,97 & 0,62 & 0,76 & 0,70 & 1,22 & 2,03 & 0,76 & $2,56^{*}$ & 0,87 \\
\hline Musulman & - & - & $54,50^{\star * \star}$ & - & - & 1,16 & 0,84 & 2,34 & - & $17,27^{\star \star \star}$ & 0,59 \\
\hline Autres & 2,28 & 0,51 & 1,17 & 5,49 & 0,33 & 1,36 & $59,94^{\star *}$ & 1,80 & $1,80^{\star}$ & 0,93 & 0,38 \\
\hline \multicolumn{12}{|l|}{ Revenu du ménage } \\
\hline$<50 \$$ & 1,00 & 1,00 & 1,00 & 1,00 & 1,00 & 1,00 & 1,00 & 1,00 & 1,00 & 1,00 & 1,00 \\
\hline $50-100 \$$ & 0,35 & 0,84 & 0,68 & 0,69 & $0,40^{* *}$ & $0,35^{\star \star \star}$ & 1,14 & $0,46^{\star *}$ & $0,68^{*}$ & 0,92 & 0,82 \\
\hline $101-200 \$$ & $0,24^{\star *}$ & $0,28^{\star \star}$ & $0,20^{*}$ & 0,47 & 0,80 & $0,34^{\star \star *}$ & 4,11 & $0,12^{\star * *}$ & $0,55^{\star}$ & $0,39^{\star *}$ & 0,66 \\
\hline$>200 \$$ & $0,14^{\star \star *}$ & $0,26^{\star *}$ & - & 0,27 & $0,50^{*}$ & $0,23^{* * *}$ & 1,19 & $0,28^{* *}$ & $0,11^{\star \star \star}$ & 0,33 & 0,50 \\
\hline \multicolumn{12}{|l|}{ Nombre d'enfants de 6-17 ans } \\
\hline $1-2$ & 1,00 & 1,00 & 1,00 & 1,00 & 1,00 & 1,00 & 1,00 & 1,00 & 1,00 & 1,00 & 1,00 \\
\hline $3-4$ & 0,90 & 1,38 & $0,41^{*}$ & 0,90 & $0,58^{*}$ & 1,13 & 0,70 & 1,30 & 0,97 & 1,15 & 0,89 \\
\hline 5 et plus & 0,42 & 1,36 & $0,13^{\star *}$ & 1,39 & $0,52^{*}$ & 1,21 & $0,25^{*}$ & $2,42^{\star *}$ & 0,93 & 0,93 & 0,57 \\
\hline \multicolumn{12}{|l|}{ Nombre de malades } \\
\hline aucun & 1,00 & 1,00 & 1,00 & 1,00 & 1,00 & 1,00 & 1,00 & 1,00 & 1,00 & 1,00 & 1,00 \\
\hline un & 1,17 & 0,71 & 1,52 & 0,87 & 0,87 & 1,46 & $2,85^{\star \star}$ & 0,82 & 1,24 & 1,21 & $2,15^{\star \star}$ \\
\hline deux & 0,43 & 0,68 & $3,07^{\star}$ & 0,45 & 1,85 & 0,87 & 1,88 & 1,09 & 1,02 & 1,06 & $2,39^{*}$ \\
\hline trois et plus & $2,84^{*}$ & 1,14 & $5,28^{\star *}$ & 2,57 & 1,40 & 1,77 & 0,40 & 1,48 & 0,70 & 0,39 & $2,90^{* *}$ \\
\hline \multicolumn{12}{|l|}{ Distance à l'école (secondaire) } \\
\hline$<2$ & 1,00 & 1,00 & 1,00 & 1,00 & 1,00 & 1,00 & 1,00 & 1,00 & 1,00 & 1,00 & 1,00 \\
\hline $2-5$ & 0,60 & 0,60 & 1,17 & 0,62 & $1,85^{\star}$ & 0,82 & $3,64^{\star \star}$ & 0,88 & $1,92^{\star}$ & 1,49 & 0,62 \\
\hline $5-10$ & - & 0,91 & 2,14 & $3,42^{\star \star}$ & 1,19 & 1,42 & 1,78 & 0,99 & $1,95^{\star *}$ & 0,78 & 1,41 \\
\hline 10 et plus & - & 0,77 & $6,46^{* * *}$ & 2,17 & $31,76^{\star \star *}$ & $2,44^{*}$ & 4,65 & 1,62 & $3,12^{* * *}$ & 1,57 & 1,46 \\
\hline Effectifs de l'échantillon (n) & 884 & 755 & 806 & 581 & 685 & 1104 & 555 & 859 & 1239 & 786 & 948 \\
\hline Pseudo R2 & 0,150 & 0,125 & 0,215 & 0,146 & 0,162 & 0,165 & 0,238 & 0,112 & 0,225 & 0,209 & 0,181 \\
\hline
\end{tabular}

Significatif au seuil de $5 \%,{ }^{* *}$ Significatif au seuil de $1 \%,{ }^{* * *}$ Significatif au seuil de $1 \% 0$

Source : Données de l'enquête auprès des ménages, EADE-RDC 2012 
Tableau A30 : Hiérarchisation des variables selon le pouvoir explicatif du risque d'être en dehors de l'école au niveau des 6-11 ans et au niveau des 12-17 ans

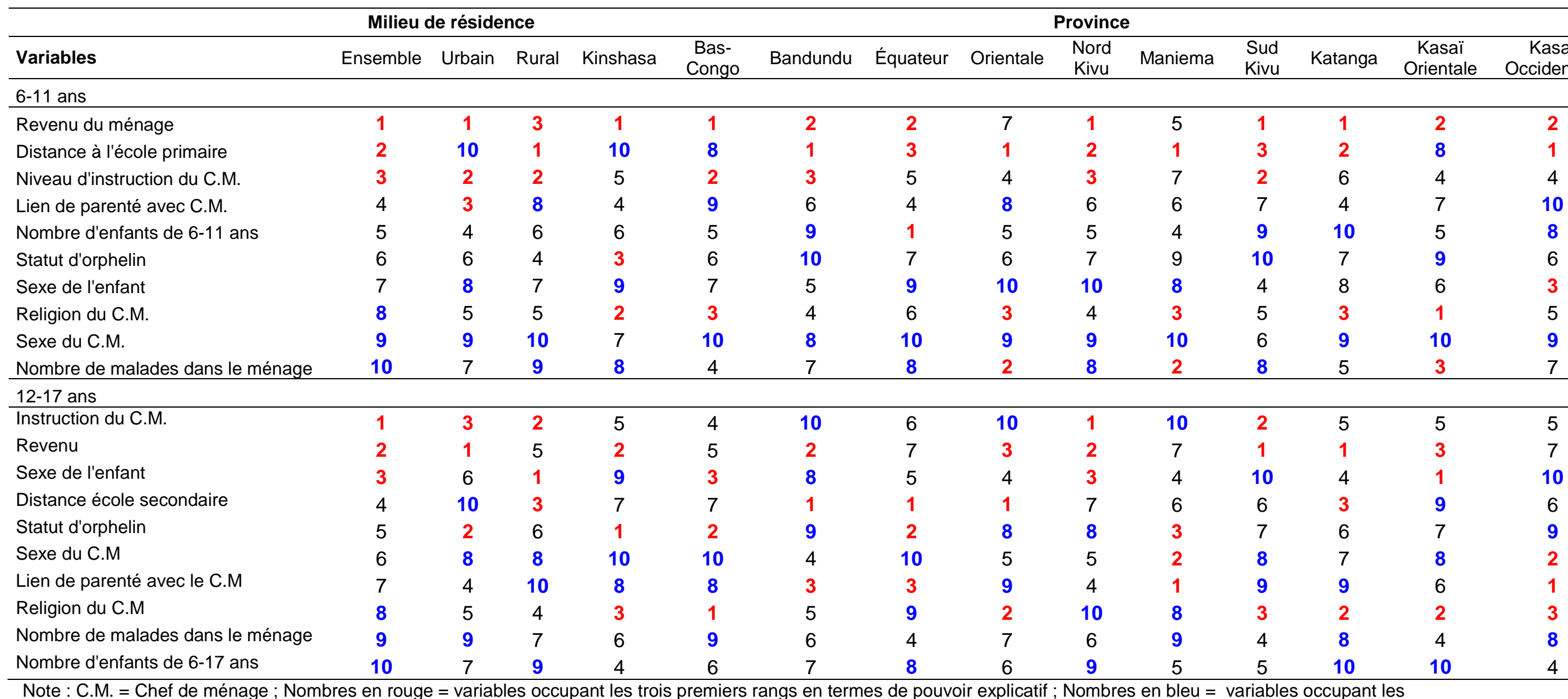

Note : C.M. = Chef de ménage $;$ Nombres en rouge = variables occupant les trois premiers rangs en termes de pouvoir explicatif $;$ Nombres en bleu = variables occupant les trois derniers rangs en termes de pouvoir explicatif.

Source : Données de l'enquête auprès des ménages, EADE-RDC 2012 
Tableau A31 : Raisons d'arrêt ou d'abandon scolaire (en \%) et raisons de non inscription à l'école (en \%) des enfants de 6-11 ans et de 12-17 ans

\begin{tabular}{|c|c|c|c|c|c|c|c|c|c|c|c|c|}
\hline Raisons d'arrêt/abandon & Kinshasa & Bas-Congo & Bandundu & Equateur & $\begin{array}{l}\text { Province } \\
\text { Orientale }\end{array}$ & Nord-Kivu & Maniema & Sud-Kivu & Katanga & $\begin{array}{c}\text { Kasaï } \\
\text { Oriental }\end{array}$ & $\begin{array}{c}\text { Kasaï } \\
\text { Occidental }\end{array}$ & RDC \\
\hline Argent & 87,4 & 61,4 & 57,6 & 68,5 & 59,7 & 84,3 & 53,8 & 89,1 & 65,5 & 70,1 & 59,9 & 71,0 \\
\hline Contraintes familliales & 19,6 & 10,2 & 8,0 & 12,8 & 11,4 & 18,8 & 5,5 & 37,3 & 15,7 & 11,7 & 20,6 & 16,5 \\
\hline Pas d'école/enseignant & 1,0 & 3,8 & 0,0 & 0,0 & 26,0 & 0,0 & 0,0 & 0,0 & 29,4 & 2,0 & 13,6 & 11,6 \\
\hline Désintérêt de l'enfant & 3,1 & 12,1 & 9,9 & 8,2 & 12,3 & 5,5 & 4,1 & 8,9 & 10,4 & 14,4 & 14,5 & 9,5 \\
\hline Décès membre du ménage & 8,8 & 3,7 & 0,5 & 6,5 & 12,8 & 10,0 & 7,6 & 5,6 & 9,5 & 8,7 & 14,1 & 9,0 \\
\hline Travaux domestiques & 1,7 & 1,0 & 12,0 & 3,0 & 5,1 & 0,9 & 4,1 & 8,9 & 4,4 & 30,6 & 24,7 & 7,7 \\
\hline Déplacement famille & 8,2 & 6,8 & 4,3 & 6,5 & 4,0 & 3,4 & 5,5 & 14,5 & 11,2 & 5,4 & 9,1 & 7,6 \\
\hline Maladie enfant & 4,4 & 5,0 & 12,0 & 4,6 & 10,5 & 4,2 & 11,0 & 11,9 & 3,6 & 8,1 & 11,9 & 6,8 \\
\hline Mauvais résultats & 4,6 & 9,1 & 9,5 & 3,7 & 7,5 & 11,4 & 6,9 & 6,2 & 4,1 & 10,4 & 4,9 & 6,7 \\
\hline Maladie membre du ménage & 7,2 & 15,5 & 3,0 & 2,0 & 6,5 & 5,5 & 5,6 & 11,2 & 5,0 & 4,9 & 8,2 & 6,6 \\
\hline Pas d'école proche & 0,0 & 0,0 & 24,0 & 0,0 & 6,0 & 10,8 & 14,0 & 8,8 & 8,4 & 2,0 & 8,2 & 6,0 \\
\hline Grossesse & 2,0 & 5,8 & 11,7 & 4,4 & 7,0 & 5,3 & 11,6 & 0,8 & 2,9 & 2,0 & 0,1 & 3,9 \\
\hline Insécurité / conflits & 0,4 & 1,9 & 0,0 & 4,2 & 4,3 & 8,1 & 1,4 & 15,7 & 2,5 & 0,4 & 4,1 & 3,9 \\
\hline Indiscipline & 3,2 & 5,0 & 4,2 & 8,6 & 4,0 & 4,6 & 1,4 & 2,3 & 3,5 & 0,9 & 2,0 & 3,7 \\
\hline Compréhension difficile & 1,1 & 5,7 & 9,6 & 1,5 & 1,7 & 3,4 & 1,4 & 3,8 & 2,9 & 9,6 & 1,1 & 3,3 \\
\hline Mariage & 0,0 & 0,0 & 3,0 & 1,5 & 4,7 & 2,1 & 10,2 & 2,2 & 3,0 & 7,6 & 8,0 & 3,3 \\
\hline Maltraitance à l'école & 1,6 & 1,1 & 12,5 & 1,5 & 1,1 & 2,1 & 0,0 & 0,8 & 3,3 & 0,4 & 2,1 & 2,1 \\
\hline Changement établissement & 2,1 & 2,9 & 9,0 & 1,0 & 1,5 & 1,5 & 0,0 & 0,4 & 0,7 & 1,4 & 2,0 & 1,6 \\
\hline Maltraitance à la maison & 0,0 & 2,8 & 0,0 & 2,3 & 2,4 & 0,3 & 1,4 & 1,0 & 1,5 & 0,5 & 2,7 & 1,3 \\
\hline Travail rémunéré & 0,5 & 0,4 & 0,0 & 0,4 & 1,1 & 0,5 & 2,8 & 0,7 & 1,0 & 0,0 & 0,0 & 0,6 \\
\hline Handicap (enfant) & 1,1 & 0,9 & 3,0 & 0,4 & 0,8 & 0,0 & 0,0 & 0,0 & 0,2 & 0,0 & 0,7 & 0,5 \\
\hline Etat nutritionnel & 0,0 & 0,0 & 0,0 & 0,0 & 0,0 & 0,2 & 0,0 & 2,9 & 0,4 & 1,6 & 0,0 & 0,4 \\
\hline A déjà fréquenté & 243382 & 97922 & 52960 & 125131 & 262831 & 182041 & 22974 & 125687 & 370765 & 159336 & 111785 & 1754814 \\
\hline \multicolumn{13}{|l|}{ Raisons de non inscription } \\
\hline Argent & 90,7 & 44,3 & 59,4 & 82,9 & 71,8 & 85,9 & 55,1 & 82,4 & 70,5 & 56,5 & 50,0 & 69,8 \\
\hline Pas d'école proche & 0,0 & 11,4 & 30,0 & 1,4 & 41,8 & 24,0 & 30,6 & 11,6 & 20,2 & 12,6 & 28,0 & 21,0 \\
\hline Trop jeune pour école & 8,8 & 64,2 & 24,2 & 2,8 & 14,0 & 15,0 & 32,8 & 10,4 & 22,6 & 5,8 & 23,2 & 18,6 \\
\hline Contraintes familliales & 21,1 & 7,3 & 16,8 & 21,6 & 19,6 & 11,9 & 16,7 & 25,5 & 12,2 & 5,5 & 17,2 & 15,1 \\
\hline Pas d'école/enseignant & 0,0 & 7,4 & 5,4 & 2,8 & 4,6 & 3,2 & 1,2 & 2,8 & 25,6 & 2,6 & 20,4 & 10,4 \\
\hline Désintérêt de l'enfant & 2,6 & 7,7 & 7,8 & 5,7 & 7,8 & 1,7 & 9,8 & 8,3 & 7,1 & 21,2 & 14,0 & 8,2 \\
\hline Travaux domestiques & 0,0 & 0,0 & 4,1 & 8,8 & 6,1 & 2,3 & 12,4 & 19,8 & 2,6 & 17,4 & 20,4 & 7,5 \\
\hline Maladie enfant & 9,6 & 5,9 & 6,4 & 7,8 & 8,0 & 1,9 & 11,6 & 8,2 & 3,0 & 17,3 & 8,0 & 6,6 \\
\hline Décès membre du ménage & 0,0 & 0,2 & 4,9 & 5,0 & 10,1 & 3,6 & 2,0 & 8,1 & 7,5 & 9,5 & 7,5 & 6,4 \\
\hline Compréhension difficile & 2,3 & 8,0 & 7,3 & 11,2 & 3,6 & 0,2 & 11,5 & 2,8 & 3,2 & 23,8 & 5,9 & 6,3 \\
\hline Insécurité / conflits & 0,0 & 0,0 & 0,0 & 0,7 & 6,3 & 15,4 & 0,6 & 10,4 & 2,8 & 0,8 & 3,5 & 4,6 \\
\hline Maladie membre du ménage & 2,9 & 7,1 & 1,5 & 7,1 & 2,3 & 3,3 & 6,2 & 6,2 & 5,2 & 4,6 & 3,3 & 4,4 \\
\hline Déplacement famille & 2,8 & 1,9 & 1,0 & 0,5 & 2,6 & 3,5 & 2,9 & 7,1 & 1,9 & 4,5 & 2,0 & 2,6 \\
\hline Indiscipline & 0,0 & 1,4 & 1,4 & 0,7 & 2,0 & 0,7 & 2,8 & 0,5 & 1,7 & 4,8 & 0,3 & 1,5 \\
\hline Handicap (enfant) & 3,4 & 1,2 & 0,0 & 1,9 & 1,3 & 1,6 & 1,2 & 2,1 & 0,9 & 0,7 & 3,2 & 1,4 \\
\hline Maltraitance à l'école & 0,0 & 0,2 & 2,0 & 0,7 & 1,5 & 0,0 & 2,3 & 1,9 & 0,9 & 0,0 & 2,2 & 1,0 \\
\hline Etat nutritionnel & 0,0 & 1,2 & 0,7 & 2,2 & 0,6 & 0,1 & 2,3 & 1,4 & 0,3 & 3,2 & 0,3 & 0,9 \\
\hline Maltraitance à la maison & 0,0 & 0,0 & 0,0 & 0,7 & 0,2 & 0,0 & 0,0 & 0,0 & 0,7 & 1,9 & 0,7 & 0,5 \\
\hline Travail rémunéré & 0,0 & 0,0 & 0,0 & 0,7 & 0,9 & 0,4 & 0,0 & 0,7 & 0,4 & 0,0 & 0,0 & 0,4 \\
\hline Mariage & 0,0 & 0,0 & 0,7 & 0,0 & 0,0 & 0,4 & 0,6 & 0,0 & 0,6 & 0,5 & 0,3 & 0,3 \\
\hline Grossesse & 0,0 & 0,0 & 2,0 & 0,0 & 0,3 & 0,0 & 0,0 & 0,5 & 0,4 & 0,0 & 0,0 & 0,3 \\
\hline N'a jamais fréquenté & 76930 & 146573 & 234746 & 277923 & 346559 & 379624 & 55797 & 189749 & 695667 & 240845 & 238740 & 2883153 \\
\hline
\end{tabular}


Tableau A32 : Répartition (en \%) des enfants âgés de 6-17 ans n'ayant jamais été à l'école et ceux déscolarisés selon les raisons de non inscription ou d'arrêt de scolarisation

\begin{tabular}{lcccccc}
\hline \multirow{2}{*}{ Raisons } & \multicolumn{3}{c}{ Raisons de non inscription } & \multicolumn{3}{c}{ Raisons d'arrêt de scolarisation } \\
\cline { 2 - 7 } & Garçons & Filles & Ensemble & Garçons & Filles & Ensemble \\
\hline Argent & 68,2 & 71,2 & 69,8 & 74,6 & 68,2 & 71,0 \\
Contraintes familiales & 13,6 & 16,5 & 15,1 & 16,1 & 16,9 & 16,5 \\
Pas d'école/enseignant & 9,1 & 11,4 & 10,4 & 18,0 & 6,4 & 11,5 \\
Désintérêt de l'enfant & 8,4 & 8,0 & 8,2 & 6,8 & 11,7 & 9,5 \\
Décès membre du ménage & 5,6 & 7,0 & 6,4 & 10,9 & 7,6 & 9,0 \\
Travaux domestiques & 4,0 & 10,6 & 7,5 & 3,8 & 10,8 & 7,7 \\
Déplacement famille & 2,7 & 2,5 & 2,6 & 7,8 & 7,4 & 7,6 \\
Maladie enfant & 5,6 & 7,5 & 6,6 & 6,4 & 7,2 & 6,9 \\
Mauvais résultats & na & na & na & 5,3 & 7,7 & 6,7 \\
Maladie membre du ménage & 3,6 & 5,0 & 4,4 & 5,5 & 7,4 & 6,5 \\
Pas d'école proche & 23,3 & 18,9 & 20,9 & 7,1 & 5,2 & 6,0 \\
Insécurité / conflits & 4,0 & 5,1 & 4,6 & 5,0 & 3,1 & 3,9 \\
Grossesse & 0,0 & 0,6 & 0,3 & 0,8 & 6,3 & 3,9 \\
Indiscipline & 1,9 & 1,2 & 1,5 & 3,4 & 3,9 & 3,7 \\
Compréhension difficile & 5,4 & 7,0 & 6,3 & 3,2 & 3,4 & 3,3 \\
Mariage & 0,0 & 0,6 & 0,3 & 0,8 & 5,2 & 3,3 \\
Maltraitance à l'école & 0,6 & 1,3 & 1,0 & 2,1 & 2,1 & 2,1 \\
Changement établissement & na & na & na & 2,1 & 1,3 & 1,6 \\
Maltraitance à la maison & 0,6 & 0,4 & 0,5 & 0,7 & 1,8 & 1,3 \\
Travail rémunéré & 0,5 & 0,3 & 0,4 & 0,7 & 0,6 & 0,6 \\
Handicap (enfant) & 1,6 & 1,2 & 1,4 & 0,8 & 0,3 & 0,5 \\
Etat nutritionnel & 1,0 & 0,9 & 0,9 & 0,5 & 0,4 & 0,4 \\
Trop jeune pour école & 21,9 & 15,7 & 18,6 & na & na & na \\
Autres raisons & 4,8 & 4,2 & 4,5 & 2,8 & 3,1 & 3,0 \\
\hline Effectifs & 1340373 & 1542781 & 2883154 & 767207 & 984124 & 1751331 \\
\hline NB & \multicolumn{7}{c}{ na } & & & & &
\end{tabular}

NB : na $=$ non applicable

Source : Données de l'enquête auprès des ménages, EADE-RDC 2012 
Tableau A33: Proportions (en \%) des EADE d'âge du primaire et du secondaire en dehors de l'école impliqués dans le travail selon les caractéristiques de l'enfant et de leur ménage

A travaillé (tout travail)

\begin{tabular}{|c|c|c|c|c|c|c|}
\hline \multirow{2}{*}{ Caractéristiques } & \multicolumn{3}{|c|}{ Proportions } & \multicolumn{3}{|c|}{ Effectifs de travailleurs } \\
\hline & Garçons & Filles & Ensemble & Garçons & Filles & Ensemble \\
\hline \multicolumn{7}{|l|}{ Age } \\
\hline 6 & 87,3 & 90,5 & 89,6 & 5239 & 13419 & 18658 \\
\hline 7 & 95,7 & 84,4 & 91,1 & 30523 & 18642 & 49165 \\
\hline 8 & 91,2 & 95,5 & 93,3 & 25686 & 26840 & 52526 \\
\hline 9 & 94,6 & 98,2 & 96,7 & 21605 & 31961 & 53566 \\
\hline 10 & 97,0 & 100,0 & 98,5 & 36603 & 35917 & 72520 \\
\hline 11 & 80,0 & 94,6 & 89,6 & 13008 & 29339 & 42347 \\
\hline 12 & 92,0 & 100,0 & 96,3 & 28453 & 36322 & 64775 \\
\hline 13 & 87,7 & 93,3 & 90,1 & 25412 & 19793 & 45205 \\
\hline \multicolumn{7}{|l|}{ Milieu } \\
\hline Urbain & 92,3 & 94,4 & 93,5 & 79142 & 102619 & 181761 \\
\hline Rural & 91,7 & 96,7 & 94,2 & 107388 & 109614 & 217002 \\
\hline \multicolumn{7}{|l|}{ Province } \\
\hline Kinshasa & 95,1 & 90,3 & 92,4 & 31326 & 37477 & 68803 \\
\hline Bas-Congo & 78,6 & 100,0 & 93,3 & 4329 & 12056 & 16385 \\
\hline Bandundu & 100,0 & 100,0 & 100,0 & 4846 & 10220 & 15066 \\
\hline Equateur & 97,4 & 100,0 & 98,8 & 18598 & 23090 & 41688 \\
\hline Orientale & 88,1 & 92,2 & 89,5 & 31131 & 16879 & 48010 \\
\hline Nord Kivu & 79,5 & 100,0 & 87,0 & 19804 & 14229 & 34033 \\
\hline Maniema & 100,0 & 87,4 & 93,8 & 3786 & 3185 & 6971 \\
\hline sud Kivu & 100,0 & 84,6 & 92,1 & 12561 & 11211 & 23772 \\
\hline Katanga & 97,2 & 95,8 & 96,5 & 42386 & 41943 & 84329 \\
\hline Kasaï oriental & 92,2 & 100,0 & 97,7 & 10666 & 27536 & 38202 \\
\hline Kasaï occidental & 82,3 & 100,0 & 93,4 & 7096 & 14405 & 21501 \\
\hline \multicolumn{7}{|c|}{ Classes de revenu annuel } \\
\hline Moins de 50 USD & 89,8 & 96,7 & 93,5 & 86185 & 109741 & 195926 \\
\hline 50-100 USD & 95,2 & 100,0 & 97,4 & 55351 & 48346 & 103697 \\
\hline 101-200 USD & 89,0 & 88,3 & 88,6 & 29980 & 36291 & 66271 \\
\hline 201-500 USD & 100,0 & 93,2 & 96,1 & 13444 & 16860 & 30304 \\
\hline Plus de 500 USD & 100,0 & 100,0 & 100,0 & 1569 & 994 & 2563 \\
\hline \multicolumn{7}{|c|}{ Sexe du chef de ménage } \\
\hline Hommes & 93,4 & 97,2 & 95,3 & 119546 & 126672 & 246218 \\
\hline Femmes & 89,5 & 93,3 & 91,6 & 66983 & 85560 & 152543 \\
\hline \multicolumn{7}{|c|}{$\begin{array}{l}\text { Niveau d'instruction du chef } \\
\text { de ménage }\end{array}$} \\
\hline N'a pas été à l'école & 89,8 & 95,3 & 92,7 & 108584 & 124610 & 233194 \\
\hline Primaire & 94,0 & 96,7 & 95,2 & 36449 & 30949 & 67398 \\
\hline Secondaire 1-2 & 96,4 & 92,1 & 94,1 & 12978 & 14715 & 27693 \\
\hline Secondaire 3-6 & 96,1 & 96,4 & 96,3 & 26404 & 37714 & 64118 \\
\hline Supérieur & 100,0 & 100,0 & 100,0 & 2114 & 4244 & 6358 \\
\hline
\end{tabular}

Source : Données de l'enquête auprès des ménages, EADE-RDC 2012 
Tableau A34 : Taux de couverture (en \%) de l'EADE-RDC 2012 par province

\begin{tabular}{|c|c|c|c|c|c|c|c|c|c|}
\hline \multirow[b]{2}{*}{ Provinces } & \multicolumn{3}{|c|}{ Ménages } & \multicolumn{2}{|c|}{ Effectifs identifiés } & \multicolumn{2}{|c|}{ Effectifs enquêtés } & \multicolumn{2}{|c|}{ Couverture } \\
\hline & Echantillon & Enquêté & $\begin{array}{l}\text { Couv } \\
\text { Men }\end{array}$ & $\begin{array}{l}-5 \\
\text { ans }\end{array}$ & $6-17$ ans & $3-5$ ans & $6-17$ ans & 3-5 ans & 6-17 ans \\
\hline Kinshasa & 1140 & 1124 & 98,6 & 559 & 1916 & 552 & 1900 & 98,7 & 99,2 \\
\hline Bas-Congo & 1140 & 1139 & 99,9 & 586 & 1718 & 572 & 1695 & 97,6 & 98,7 \\
\hline Bandundu & 1140 & 1135 & 99,6 & 655 & 1829 & 650 & 1824 & 99,2 & 99,7 \\
\hline Equateur & 1020 & 1009 & 98,9 & 614 & 1439 & 611 & 1435 & 99,5 & 99,7 \\
\hline $\begin{array}{l}\text { Province } \\
\text { Orientale }\end{array}$ & 1080 & 1078 & 99,8 & 568 & 1582 & 568 & 1580 & 100,0 & 99,9 \\
\hline Nord-Kivu & 1410 & 1400 & 99,3 & 910 & 2514 & 910 & 2493 & 100,0 & 99,2 \\
\hline Maniema & 960 & 957 & 99,7 & 577 & 1350 & 575 & 1345 & 99,7 & 99,6 \\
\hline Sud-Kivu & 1230 & 1229 & 99,9 & 725 & 1944 & 723 & 1937 & 99,7 & 99,6 \\
\hline Katanga & 1920 & 1916 & 99,8 & 1146 & 3010 & 1133 & 2970 & 98,9 & 98,7 \\
\hline Kasaï Oriental & 1230 & 1198 & 97,4 & 698 & 1882 & 699 & 1876 & 100,1 & 99,7 \\
\hline $\begin{array}{l}\text { Kasaï } \\
\text { Occidental }\end{array}$ & 1350 & 1340 & 99,3 & 834 & 2277 & 830 & 2260 & 99,5 & 99,3 \\
\hline $\mathrm{RDC}$ & 13620 & 13525 & 99,3 & 7872 & 21461 & 7823 & 21315 & 99,4 & 99,3 \\
\hline
\end{tabular}

Source : Données de l'enquête auprès des ménages, EADE-RDC 2012 


\section{Annexe 5 : L'échantillonnage}

\section{Calcul des probabilités d'inclusion (EADE)}

\section{Mode de tirage}

Les unités primaires (quartiers ou cités ou secteurs/chefferies, segments), sont tirées avec des probabilités proportionnelles à la taille (effectifs de population). En cas de segmentation, les segments sont tirés avec probabilité proportionnelle à la taille. Les unités secondaires, tertiaires sont tirées avec probabilités égales. Par exemple les ménages ont été sélectionnés par un tirage systématique à probabilités égales. Quand un village ou un quartier est tiré, on procède à un dénombrement des ménages et on procède éventuellement à la segmentation. Si le nombre de ménages compté est inférieur ou égal à 500 , on tire les 30 ménages. Si le nombre de ménages comptés est supérieur à 500 on procède à la segmentation qui est un découpage en des segments d'environ 300 ménages. On tire ensuite un segment et dans ce segment on tire les 30 ménages.

\section{Cas des villes statutaires}

- Au $1^{\text {er }}$ degré (unité primaire de sondage : UPS) tirage des quartiers proportionnellement à leur taille en population. Un comptage rapide permettra de déterminer la taille en ménages de chaque unité primaire et d'en dresser une liste. Si cette taille est inférieure à 500 ménages on procédera au tirage des ménages.

$2^{\text {ème }}$ degré : Si cette taille est supérieure à 500 ménages on procédera à la segmentation (constitution de segments de 300 ménages environ). Un seul segment sera choisi de façon aléatoire (tirage proportionnel à la taille des segments) sur lequel on procèdera au tirage des ménages.

- Au 3è degré on procédera au tirage des ménages. Un nombre fixe de ménages (30 ménages) seront tirés aléatoirement (tirage systématique à probabilités égales) dans le segment échantillon.

Au premier degré : tirage aléatoire de quartiers dans la strate

La probabilité qu'un quartier $Q_{i}$ soit échantillonné dans une state $\mathbf{s}$ est :

$$
p_{(Q i) s}=Q_{s^{*}} \frac{M_{s i}}{\sum_{i=1}^{N_{s}} M_{s i}}=Q_{s} * \frac{M_{s i}}{P_{s}}
$$

$Q_{s}$ est le nombre total de quartiers échantillonnés dans la strate $\mathbf{S}$

$M_{\mathrm{si}}$ est la taille estimée du quartier i dans la strate $\mathbf{s}$

Ce nombre est la taille de la population des quartiers de la base de sondage utilisé par MICS 2010.

$\mathrm{N}_{\mathrm{s}}$ est le nombre total de quartiers dans la strate $\mathrm{s}$

$\mathrm{P}_{\mathrm{s}}$ est la population totale de la strate. 
Au $2^{\text {ème }}$ degré : tirage d'un segment

$$
p_{(s e g j)}=\frac{S_{s i j}}{\sum_{j=1}^{N_{i}} S_{s i j}}=\frac{S_{s i j}}{P_{s i}}
$$

$P_{(\text {segj) }}$ est la probabilité de tirage du segment $\mathbf{j}$ dans le quartier $\mathbf{i}$ de la strate $s S_{\text {sij }}$ est la taille $d u$ segment

$\mathrm{N}_{\mathrm{i}}$ est le nombre de segment

$\mathrm{P}_{\mathrm{si}}$ est la population totale du quartier $\mathbf{i}$

Au $3^{\text {ème }}$ degré : tirage d'un nombre fixe de ménages dans le segment tiré (30 ménages). Cette probabilité est :

$$
p_{(\text {menage }) \text { sijk }}=\frac{m_{s i j}}{M_{s i j}} \quad \text { [Équation 2] }
$$

$m_{\mathrm{sij}}$ est le nombre de ménages échantillons $(=30)$ dans le segment $\mathbf{j}$ du quartier i de la strate $\mathbf{s}$

$\mathrm{M}_{\mathrm{sij}}$ est le nombre de ménages dans le segment j du quartier i de la strate s (Compté au moment de l'enquête)

La probabilité finale est le produit des trois probabilités.

\section{Cas des cités}

Pour le cas des cités, il y a un niveau supplémentaire de tirage qui est introduit.

- Au $1^{\text {er }}$ degré on procède au tirage des cités proportionnellement à leur taille en population.

- Au $2^{\text {ème }}$ degré on procède au tirage du quartier dans la cité échantillonnée (tirage à probabilités égales). Un comptage rapide permet de déterminer la taille en ménages du quartier. Si cette taille est inférieure à 500 ménages on procédera au tirage des ménages.

Si cette taille est supérieure à 500 ménages on procédera à la segmentation (constitution de segments de 300 ménages environ). Un seul segment sera choisi de façon aléatoire sur lequel on procèdera au tirage des ménages.

- Au $3^{\text {eme }}$ degré on procédera au tirage des ménages dans le quartier/segment. Un nombre fixe de ménages (30 ménages) seront tirés aléatoirement dans chaque segment. 
Au premier degré : tirage aléatoire de cités dans la strate

La probabilité qu'une cité $C_{i}$ soit échantillonné dans une state $\mathbf{s}$ est :

$$
p_{(C i) s}=C_{s^{*}} \frac{M_{s i}}{\sum_{i=1}^{N_{s}} M_{s i}}=C_{s} * \frac{M_{s i}}{P_{s}}
$$

$\mathrm{C}_{\mathrm{s}}$ est le nombre total de cités échantillonnés dans la strate $\mathbf{s}$

$M_{\mathrm{si}}$ est la taille estimée de la cité $\mathbf{i}$ dans la strate $\mathbf{s}$

Ce nombre est la taille de la population de la cité de la base de sondage utilisé par MICS 2010.

$\mathrm{N}_{\mathrm{s}}$ est le nombre total de cités dans la strate $\mathrm{s}$

$P_{s}$ est la population totale de la strate.

Au $2^{\text {ème }}$ degré : tirage du quartier dans la cité

La probabilité qu'un quartier soit tiré dans une cité est :

$$
p_{(Q i)}=\frac{n_{s i}}{N_{s i}}
$$

$n_{s i}$ est le nombre de quartiers échantillons dans la cité i de la strate $\mathbf{s}$

(en général c'est un seul quartier qui est tiré. Donc $n_{s i}=1$ )

$\mathrm{N}_{\mathrm{si}}$ est le nombre de quartier dans la cité i de la strate $\mathrm{s}$

Au $3^{\text {ème }}$ degré : segmentation du village : probabilité de tirer le segment $k$ du quartier $j$ de la cité i dans la strate $s$

$$
p_{(s e g) k}=\frac{S_{s i j k}}{\sum_{k=1}^{N_{j}} S_{s i j k}}=\frac{S_{s i j k}}{P_{s i j}}
$$

$\mathrm{P}_{(\mathrm{seg}) \mathrm{k}}$ est la probabilité de tirage du segment $\mathbf{k}$ dans le quartier $\mathrm{j}$ de la cité $\mathrm{i}$ de la strate $\mathrm{s}$ $S_{\text {sijk }}$ est la taille du segment tiré $\mathrm{N}_{\mathrm{j}}$ est le nombre de segment du quartier $\mathrm{j}$

$\mathrm{P}_{\mathrm{sij}}$ est la population totale du quartier $\mathrm{j}$ 
Au $4^{\text {ème }}$ degré : tirage de 30 ménages dans le segment tiré

$$
p_{{\text {(ménage })_{k}}}=\frac{u_{\text {sijk }}}{U_{\text {sijk }}}
$$

$\mathrm{u}_{\mathrm{sijk}}$ est le nombre de ménages échantillons $(=30)$ dans le segment $\mathrm{k}$ du quartier $\mathbf{j}$ de la cité i de la strate $\mathbf{s}$

$\bigcup_{\text {sijk }}$ est le nombre total de ménage dans le segment $\mathrm{k}$ du quartier j de la cité i de la strate s. Ce nombre est obtenu lors de l'enquête (énumération des ménages).

La probabilité totale est égale au produit des probabilités aux différents niveaux.

\section{Cas du milieu rural}

- Au 1er degré (unité primaire de sondage : UPS). On procèdera comme précédemment pour tirer les secteurs/chefferies proportionnellement à leur taille en population.

- Au 2è degré (unité secondaire de sondage : USS). Dans chaque UPS on tirera un village (tirage à probabilités égales). Un décompte du nombre de ménages sera fait dans l'USS et on dressera une liste détaillée. On procédera à la segmentation si nécessaire

- Au 3è degré (unité tertiaire). A partir de la liste des ménages dressée ci-dessus on tirera un nombre fixe de 30 ménages (à probabilité égales).

Au premier degré : tirage aléatoire de secteurs/chefferies dans la strate

La probabilité qu'un secteur/chefferie i soit échantillonné dans une state $\mathbf{s}$ est :

$$
p_{s i}=m_{s^{*}} \frac{M_{s i}}{\sum_{i=1}^{N s} M_{s i}}=m_{s} * \frac{M_{s i}}{P_{s}}
$$

$\mathrm{m}_{\mathrm{s}}$ est le nombre total de secteurs/chefferies échantillonnés dans la strate $\mathbf{s}$

$M_{\mathrm{si}}$ est la taille estimée du secteur/chefferie i dans la strate $\mathbf{s}$

Ce nombre est la taille de la population des secteurs/chefferies de la base de sondage utilisée par MICS 2010.

Ns est le nombre total de secteur/chefferie dans la strate $s$

$\mathrm{P}_{\mathrm{s}}$ est la population totale de la strate. 
Au $2^{\text {ème }}$ degré : tirage d'un village dans la secteur/chefferie

La probabilité qu'un village soit tiré dans un secteur/chefferie. Cette probabilité est :

$p_{s i j}=\frac{n_{s i}}{N_{s i}}$

$n_{s i}$ est le nombre de villages échantillons dans la chefferie $\mathbf{i}$ de la strate $\mathbf{s}$. En général $n_{s i}=1$

$\mathrm{M}_{\mathrm{sij}}$ est la taille estimé du village j de la chefferie i de la strate $\mathbf{s}$

$\mathrm{N}_{\mathrm{si}}$ est I nombre de village dans la chefferie i de la strate $\mathrm{s}$

Au $3^{\text {ème }}$ degré segmentation du village : probabilité de tirer le segment $k$ du village j de la chefferie $i$ dans la strate $s$

$$
p_{(s e g) k}=\frac{S_{s i j k}}{\sum_{k=1}^{N_{j}} S_{s i j k}}=\frac{S_{s i j k}}{P_{s i j}}
$$

$\mathrm{P}_{(\text {seg)k }}$ est la probabilité de tirage du segment $\mathbf{k}$ dans le village j du secteur/chefferie $\mathrm{i}$ de la strate $\mathrm{s}$ (si cette probabilité est proportionnelle à la taille du segment)

$\mathrm{S}_{\text {sijk }}$ est la taille du segment tiré

$\mathrm{N}_{\mathrm{j}}$ est le nombre de segment du village $\mathrm{j}$

$P_{\text {sij }}$ est la population totale du village $j$

Au $4^{\text {ème }}$ degré : tirage de 30 ménages dans le segment tiré

$$
p_{(\text {ménage })_{k}}=\frac{u_{s i j k}}{U_{s i j k}}
$$

$\mathrm{u}_{\mathrm{sijk}}$ est le nombre de ménages échantillons $(=30)$ dans le segment $\mathrm{k}$ du village $\mathbf{j}$ de la chefferie $\mathbf{i}$ de la strate $\mathbf{s}$

$\bigcup_{\mathrm{sijk}}$ est le nombre total de ménage dans le segment $\mathrm{k}$ du village $\mathrm{j}$ de la chefferie i de la strate $\mathrm{s}$. Ce nombre est obtenu lors de l'enquête (énumération des ménages).

La probabilité totale est égale au produit des probabilités aux différents niveaux. 
For more information:

Visit our Website: www.unicef.org

\section{Or contact:}

Ministère de l'Enseignement Primaire, Secondaire et Professionnel,

Croisement des Avenues Batetela et des Cliniques

Commune de la Gombe, Kinshasa, République Démocratique du Congo.

Tel : (243) 998336049 / (243) 998472671

E-mail : ministereepsp@eduquepsp.cd / ministereepsp@yahoo.fr

Internet : http://www.eduquepsp.cd

\section{ou}

\section{I'UNICEF/RDC}

Concession IMMOTEX, 372 Ave. Col Mondjiba, Kintambo, Kinshasa, République Démocratique du Congo.

Tél : (243) 996050346

E-mail : cbaldeh@unicef.org

\section{ISSP}

03 BP 7118 Ouagadougou 03

Tél. : (226) 503025 58/59

Fax : (226) 50302560

E-mail : directeur@issp.bf

Internet : http://www.issp.bf
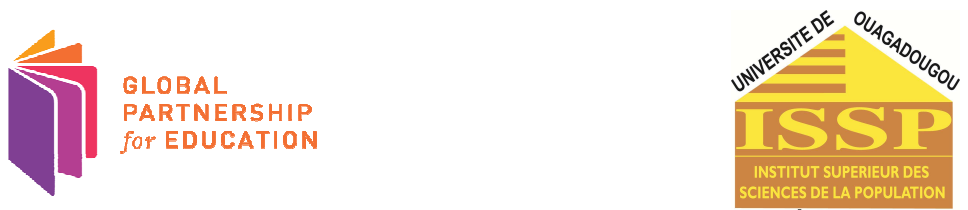